\title{
CHARACTERIZATION OF MAJOR CHROMATIN ASSEMBLY PROTEINS IN TETRAHYMENA THERMOPHILA USING PROTEOMIC AND MOLECULAR EVOLUTIONARY ANALYSES
}

\author{
by \\ Syed Nabeel Haider Shah \\ Bachelor of Science, Biology \\ Ryerson University, 2011
}

\begin{abstract}
A thesis presented to Ryerson University
in partial fulfillment of the requirements for the degree of

Master of Science

in the program of Molecular Science
\end{abstract}

Toronto, Ontario, Canada

(c) Syed Nabeel Haider Shah, 2014 


\section{Author's Declaration}

I hereby declare that I am the sole author of this thesis or dissertation.

I authorize Ryerson University to lend this thesis or dissertation to other institutions or individuals for the purpose of scholarly research.

Syed Nabeel Haider Shah

I further authorize Ryerson University to reproduce this thesis or dissertation by photocopying or by other means, in total or in part, at the request of other institutions or individuals for the purpose of scholarly research.

Syed Nabeel Haider Shah 


\section{CHARACTERIZATION OF MAJOR CHROMATIN ASSEMBLY PROTEINS IN TETRAHYMENA THERMOPHILA USING PROTEOMIC AND MOLECULAR EVOLUTIONARY ANALYSES}

Syed Nabeel Haider Shah, Master of Science, Molecular Science, Ryerson University, 2014

\section{Abstract}

Histones $\mathrm{H} 3 / \mathrm{H} 4$ are deposited onto DNA in a replication-dependent or independent fashion by the CAF1 and HIRA protein complexes. Despite the identification of these protein complexes, mechanistic details remain unclear. Recently, we showed that in $T$. thermophila histone chaperones $\mathrm{Nrp1}$, Asf1 and the $\operatorname{Imp} \beta 6$ importin $\beta$ function together to transport newly synthesized $\mathrm{H} 3 / \mathrm{H} 4$ from the cytoplasm to the nucleus. To characterize chromatin assembly proteins in T.thermophila, I used affinity purification combined with mass spectrometry to identify protein-protein interactions of Nrp1, Cac2 subunit of CAF1, HIRA and histone modifying Hat1-complex in T. thermophila. I found that the three-subunit T.thermophila CAF1 complex interacts with Casein Kinase 2 (CKII), possibly accounting for previously reported human CAF1phosphorylation. I also found that Hat2 subunit of HAT1 complex is also shared by CAF1 complex as its Cac3 subunit. This suggests that Hat2/Cac3 might exist in two separate pools of protein complexes. Remarkably, proteomic analysis of Hat2/Cac3 in turn revealed that it forms several complexes with other proteins including SIN3, RXT3, LIN9 and TESMIN, all of which have known roles in the regulation of gene expression. Finally, I asked how selective forces might have impacted on the function of proteins involved in $\mathrm{H} 3 / \mathrm{H} 4$ transport. Focusing on NASP which possesses several TPR motifs, I showed that its protein-protein interactions are conserved in T. thermophila. Using molecular evolutionary methods I show that different TPRs in NASP evolve at different rates possibly accounting for the functional diversity observed among different family members. 


\section{Acknowledgement}

In The Name of Allah, The Most Beneficent, The Most Merciful. I must thank Him for blessing me with the passion and the courage to complete this work. His guidance and blessing has always remained with me throughout the most difficult situations of my life.

I would like to thank many people who have helped me to make this journey an excellent learning experience. First of all, I would like to express my sincere appreciation and gratitude to my supervisor Dr. Jeffrey Fillingham. I joined his laboratory as an undergraduate thesis student and continued my work as a graduate student under his supervision. His support and guidance on each and every aspect of my research has remained invaluable. I consider Dr. Fillingham not only as my research mentor but also a great friend who helped me to succeed in my master's research through his stimulating guidance. Second, I would like to extend my deep and sincere appreciation to Dr. Ronald E. Pearlman. I thank him for his guidance and for making equipment and facilities at York University accessible for me.

Third, I thank my dear friend and an invaluable colleague Kanwal Ashraf for her unconditional help and support throughout this project. It would have never been possible to come this far in my research without her help. Fourth, I respectfully acknowledge Dr. Jyoti Garg (York University) for being a wonderful colleague and a helpful friend. I also thank Dr. Takahiko Akematsu (York University) for his generous help and discussions at various occasions. Fifth, special thanks to Dr. Jean Philippe Lambert from Gingras laboratory at the Lunenfeld-Tanenbaum Research Institute for his continuous assisstance with mass spectrometry.

Sixth, I would like to thank lab members, particularly, Mathew Cadorin, Nora Dannah and Renu Jeyapala for all those laughs that made this journey memorable. I also thank my 
supervisory committee members Drs. Roberto Botelho and Costin Antonescu for their timely feedback. My gratitude extends to the faculty, especially Dr. Marie Killeen, and staff of the Department of Chemistry and Biology of Ryerson University for always being there to assist me.

Finally, I would like to thank my family in back home for their time to time encouragement. Particularly, my mother, younger sister and brother have always been very supportive. Special thanks to my aunt Bibi Jan for being extremely inspirational and helpful. Her encouragement has always helped me to overcome the most difficult times in my life. Lastly, I dedicate my work to my late father who would have been very happy today if he was alive. 


\section{Table of Contents}

CHARACTERIZATION OF MAJOR CHROMATIN ASSEMBLY PROTEINS IN TETRAHYMENA THERMOPHILA USING PROTEOMIC AND MOLECULAR

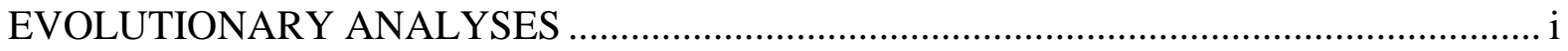

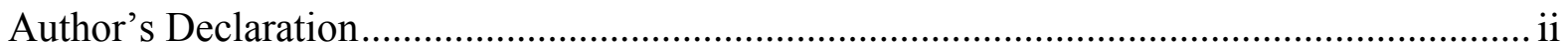

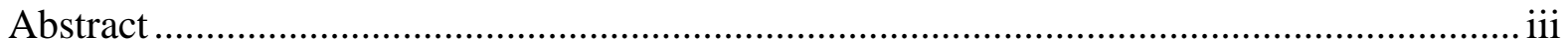

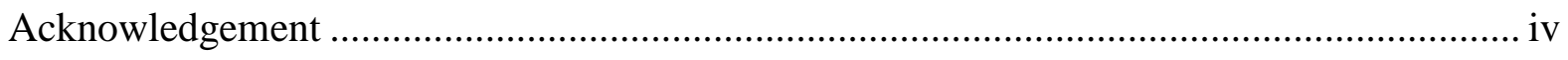

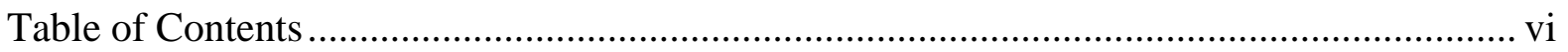

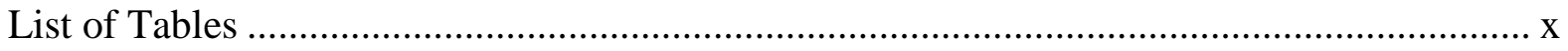

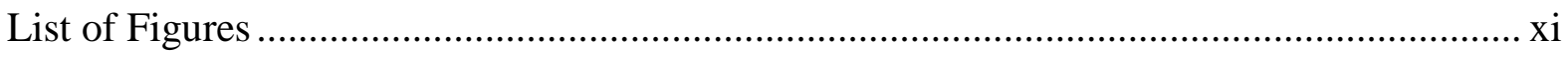

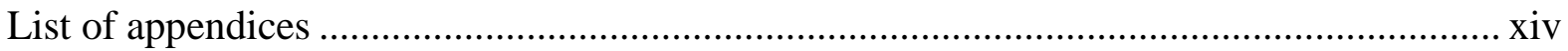

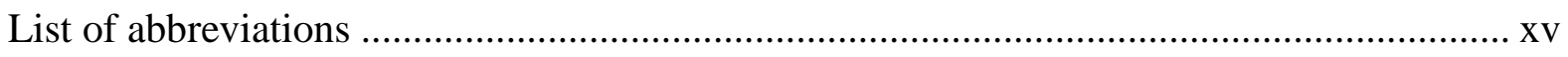

\section{PART1: PROTEOMIC ANALYSIS OF MAJOR CHROMATIN ASSEMBLY PROTEINS}

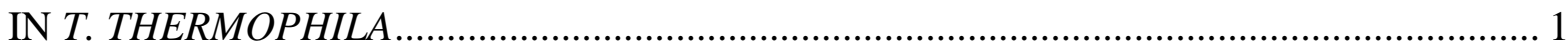

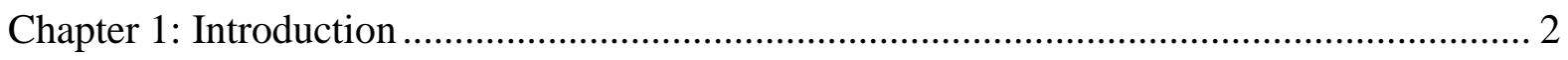

1.1- Chromatin assembly and Histone chaperones ...................................................... 2

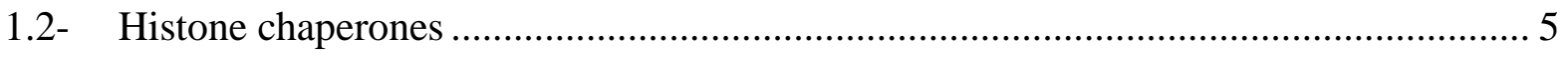

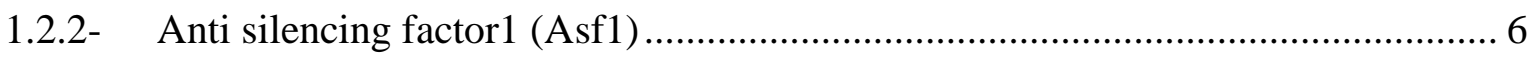

1.2.3- Nuclear autoantigenic sperm protein (NASP) …............................................ 7

1.2.4- Histone Regulatory Homolog A (HIRA) ....................................................... 9

1.2.5- Chromatin Assembly Factor 1 (CAF1) ........................................................ 10

1.3- Histone posttranslation modifications............................................................... 13

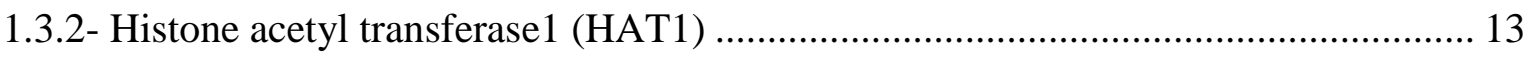

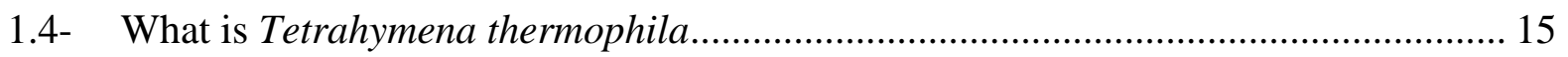

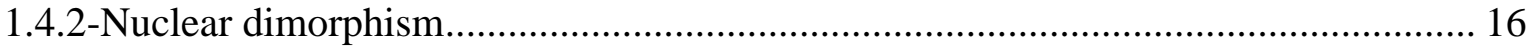

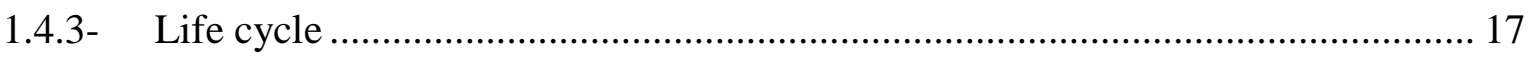

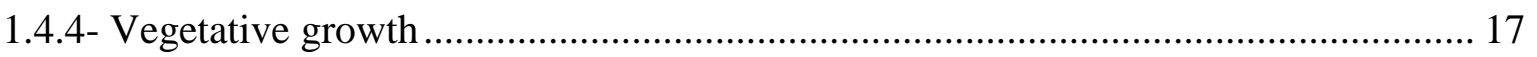

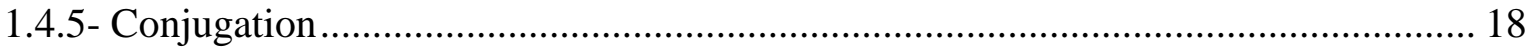

1.4.6- Programmed DNA rearrangements.............................................................. 20

1.4.7- T. thermophila as a model organism.......................................................... 22

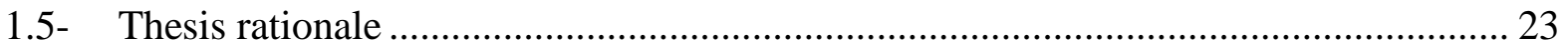

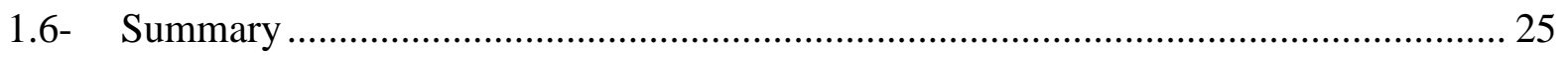




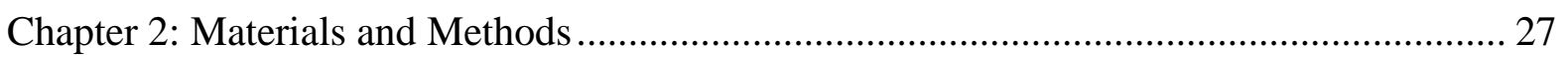

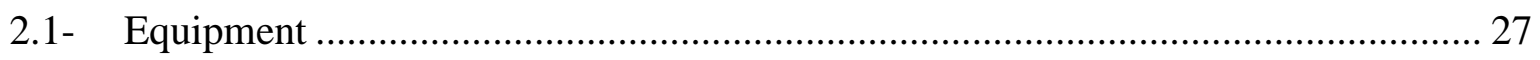

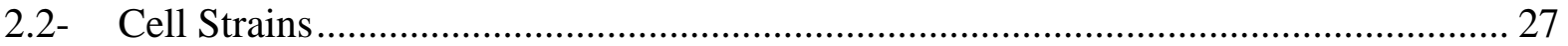

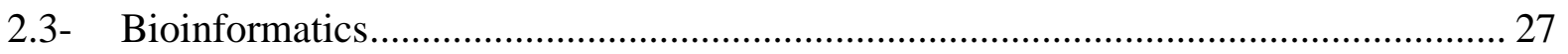

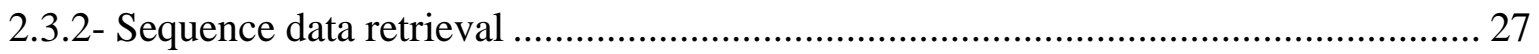

2.3.3- Multiple sequence alignments, structural predictions and gene networks................ 28

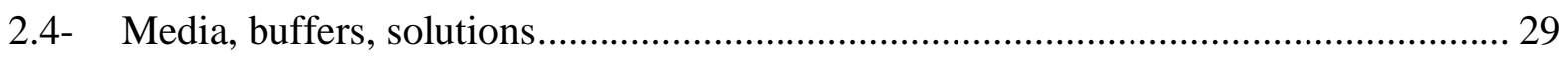

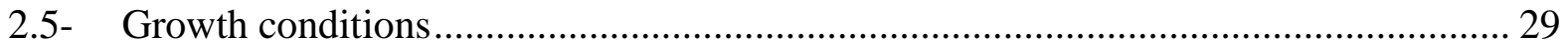

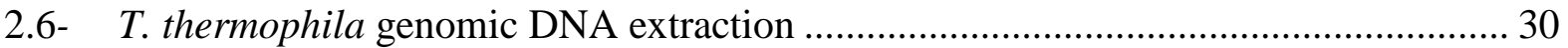

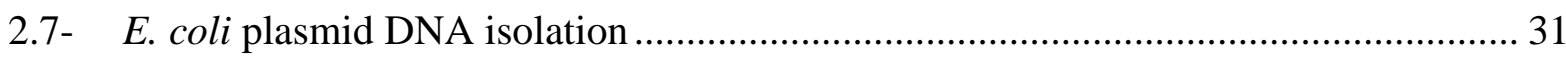

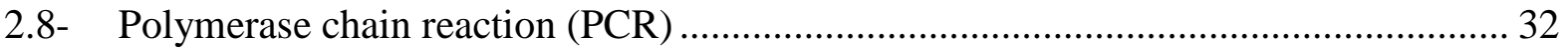

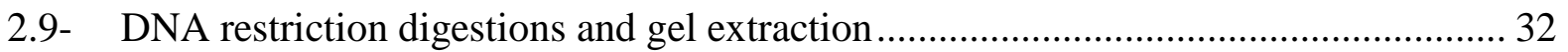

2.10- DNA ligation and transformation into competent E. coli ....................................... 33

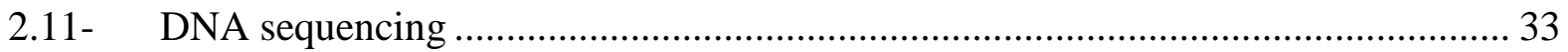

2.12- Construction of 3xFLAG-TEV-ZZ (FZZ) gene targeting vectors ............................ 33

2.13- Biolistic transformation of $T$. thermophila ............................................................ 34

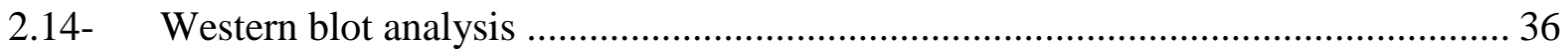

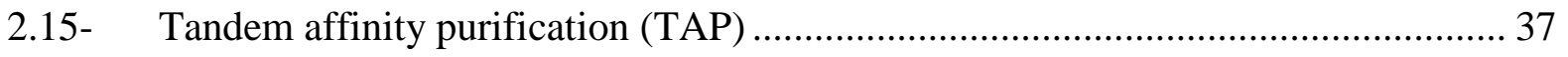

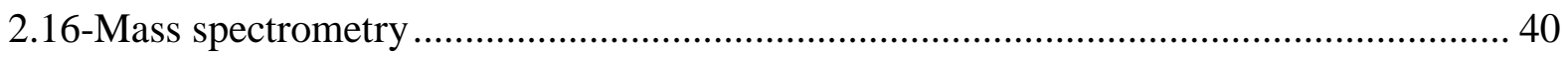

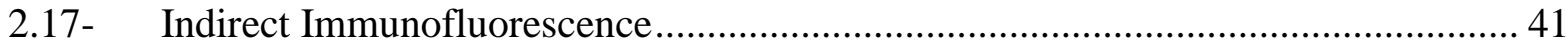

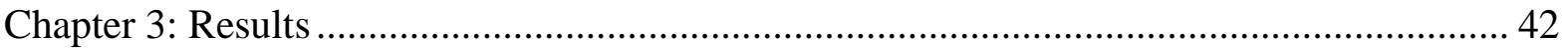

3.1- Characterization of Nrp1 ................................................................................... 44

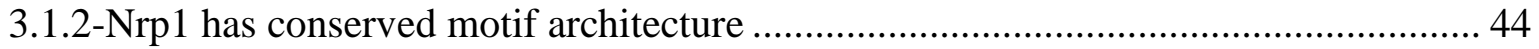

3.1.3- Nrp1 Protein-Protein interactions in growing cells ................................................ 49

3.1.4-Nrp1 localization in MIC is cell cycle dependent...................................................... 51

3.2- Characterization of HIRA in T. thermophila ............................................................. 54

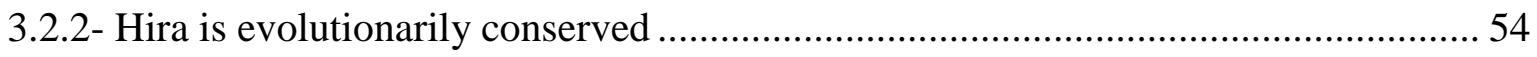

3.2.3- Proteomic analysis of T. thermophila Hira......................................................... 58

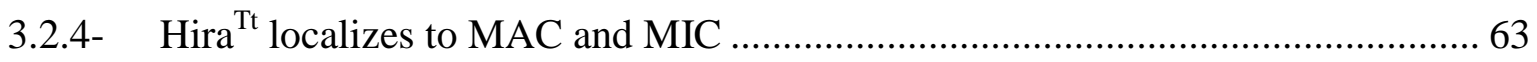

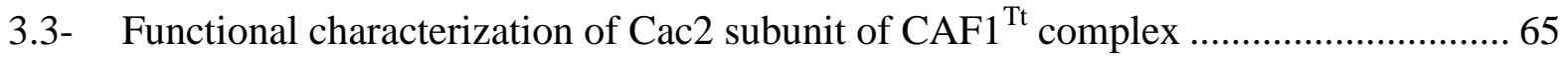

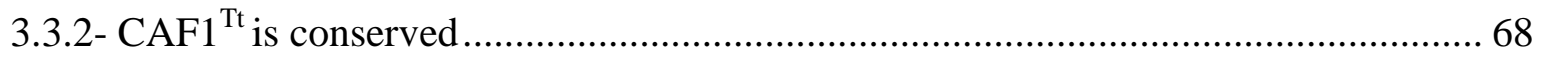

3.3.3- T. thermophila Cac2 localizes predominantly to MIC............................................... 71 
3.4- Functional characterization of Hat1 in T. thermophila ........................................... 73

3.4.2- T. thermophila Hat1-complex is conserved................................................... 77

3.4.3- T. thermophila Hat1 localizes to MAC only ....................................................... 79

3.5- Functional characterization of $T$. thermophila Hat2/Cac3 ....................................... 81

3.5.2- Hat $2 / \mathrm{Cac}^{\mathrm{Tt}}$ is a component of multiple complexes ...................................... 87

3.5.3- Hat $2 / \mathrm{Cac}^{\mathrm{Tt}}$ localization in MIC is cell cycle dependent ................................. 92

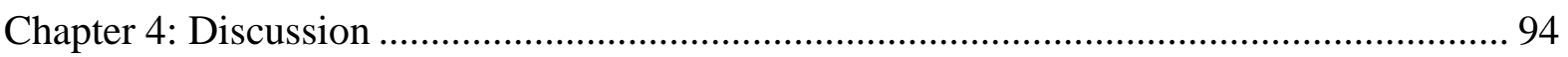

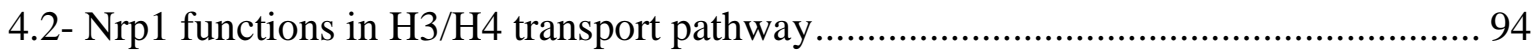

4.3- Hira is conserved in T. thermophila ..................................................................... 98

4.4- Deposition-related histone acetylation in $T$. thermophila .................................... 100

4.5- T. thermophila Hat1 and Caf1 complexes share Hat2/Cac3 subunit ....................... 102

4.6- T. thermophila Caf1 interacts with CKII complex ............................................. 103

4.7 - Functional insights into $\mathrm{CAF} 1^{\mathrm{Tt}}$ complex ...................................................... 104

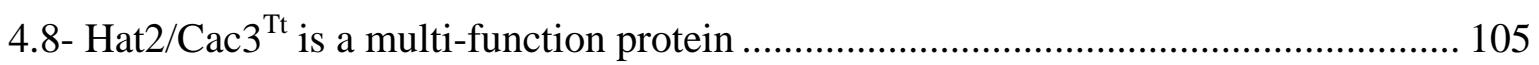

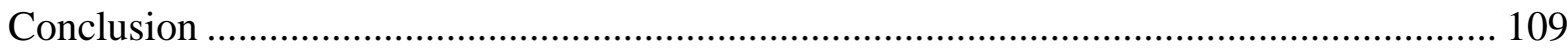

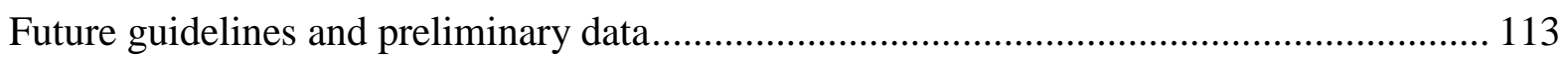

Conserved Asf1-importin $\beta$ physical interaction in growth and sexual development in the

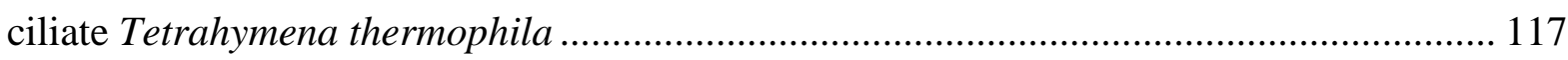

Part 2: Molecular Evolutionary analysis of H3/H4 chaperone NASP ............................... 118

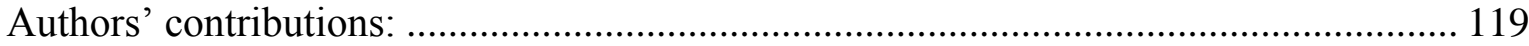

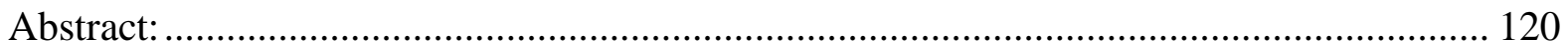

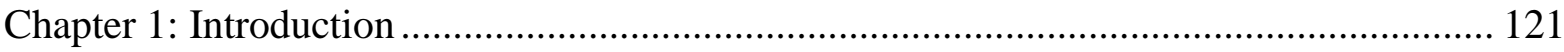

Molecular evolution of NASP and conserved histone H3/H4 transport pathway ............... 126

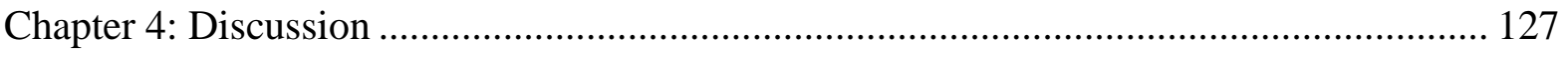

4.1- Selective constraints operating on NASP ................................................... 128

4.2- Conserved Pathway of H3/H4 Transport .................................................... 130

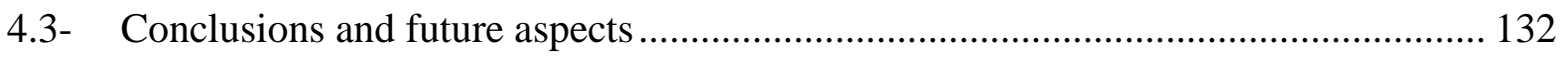

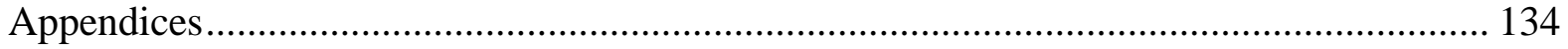

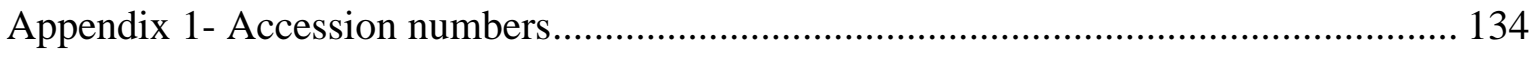

Appendix 2- A: ClustalX default color legends .................................................... 135

Appendix 2- B: Acetylated Lysine amino acid ...................................................... 135

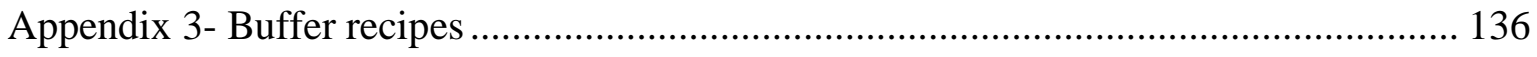


Appendix 4- Primers ........................................................................................... 138

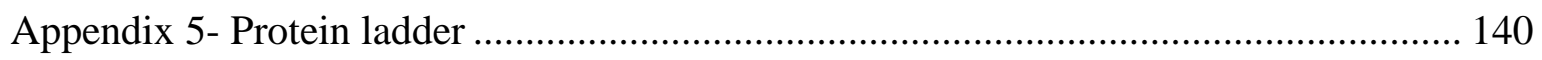

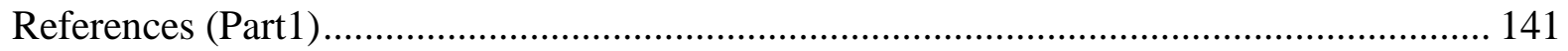

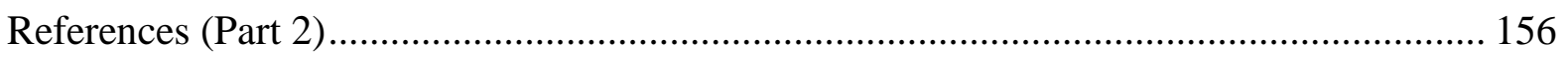




\section{List of Tables}

Table 1: Table summarizes the PCR conditions used to amplify T. thermophila gene loci...32

Table 2: Nrp1 AP-MS raw data filtered using SAINT analysis......................................51

Table 3: Cac2 ${ }^{\mathrm{Tt}}$ AP-MS data filtered using SAINT analysis............................................70

Table 4: Hat $1^{\mathrm{Tt}}$ AP-MS data filtered using SAINT analysis..............................................79

Table 5: AP-MS using Hat2/Cac3Tt-FZZ as a bait.....................................................91 


\section{List of Figures}

Figure 1: Schematic representation cytoplasmic of events during the transport of histones

$\mathrm{H} 3 / \mathrm{H} 4$ to the nucleus.

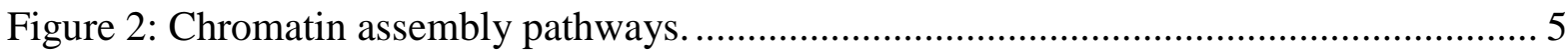

Figure 3: Vegetative T. thermophila stained with DAP1 to demonstrated two nuclei........... 17

Figure 4: Schematic representation of accelerated phenotypic assortment.. ...................... 18

Figure 5: Scan-RNA model for IES elimination in T. thermophila .................................... 22

Figure 6: Schematic representation of gene tagging vector construction. .......................... 43

Figure 7: NASP TPR motif architect across various eukaryotic lineages. ......................... 45

Figure 8: Multiple sequence alignments of TPRs 1-4 shown in A-D respectively. .............. 46

Figure 9: Predicted structure of Nrp1 is shown in ribbon representation........................... 48

Figure 10: Western blot of Nrp1-FZZ indicating the successful expression of tagged protein.

Figure 11: Successful recovery of purified Nrp1 protein detected by Western blot. 50

Figure 12: Indirect immunofluorescence of Nrp1-GFP (A) and untagged cells (B) using antiGFP antibody. 53

Figure 13: Structural comparison of various Hira homologs......................................... 55

Figure 14: Predicted tertiary structure of $T$. thermophila Hira protein............................... 57

Figure 15: Western blot of Hira-FZZ indicating the successful expression of the tagged

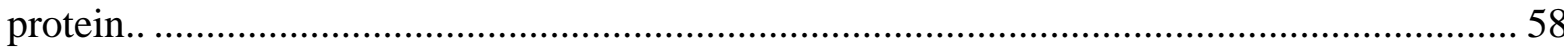

Figure 16: Western blots probed with anti-FLAG antibody indicating the successful recovery of affinity purified Hira ${ }^{\mathrm{Tt}}$. 59

Figure 17: Gene networks Hira homologs in T. thermophila and H. sapiens. 62 
Figure 18: Indirect immunofluorescence of $\operatorname{Hira}^{\mathrm{Tt}}$-FZZ (A-C) and untagged cells (D-E) using

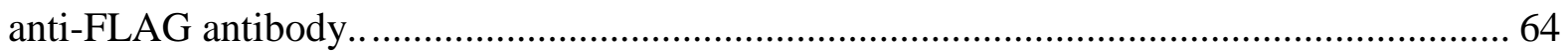

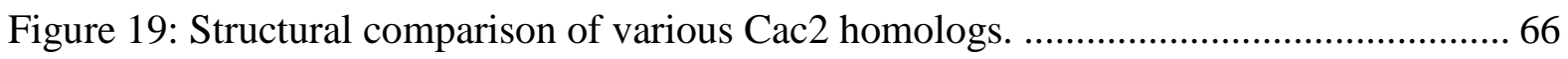

Figure 20: Multiple sequence alignments of Cac2 B-domain across various lineages.......... 66

Figure 21: Predicted tertiary structure of T. thermophila Cac2 protein............................... 67

Figure 22: Western blot of Cac2 ${ }^{\mathrm{Tt}}-\mathrm{FZZ}$ indicating the successful expression of the tagged

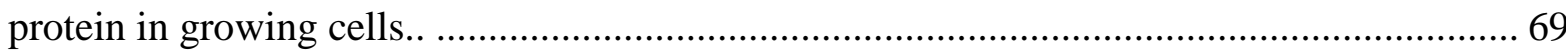

Figure 23: Successful recovery of purified Cac $2^{\mathrm{Tt}}$-FZZ protein detected by Western blot.... 70 Figure 24: Indirect immunofluorescence of $\mathrm{Cac} 2^{\mathrm{Tt}}-\mathrm{FZZ}$ and untagged cells using anti-FLAG

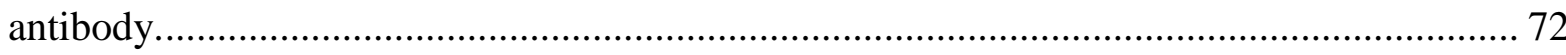

Figure 25: Indirect immunofluorescence of Asf $1^{\mathrm{Tt}}$-FZZ and untagged cells using anti-FLAG

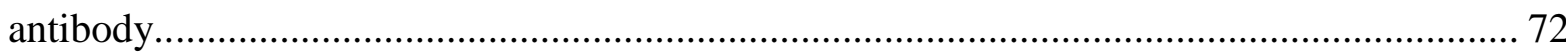

Figure 26: Predicted tertiary structure of $T$. thermophila Hat1 protein.............................. 75

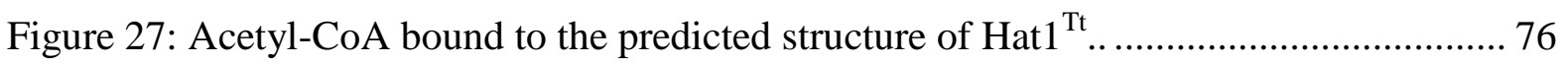

Figure 28: Western blot of Hat $1^{\mathrm{Tt}}$-FZZ indicating the successful expression of the tagged

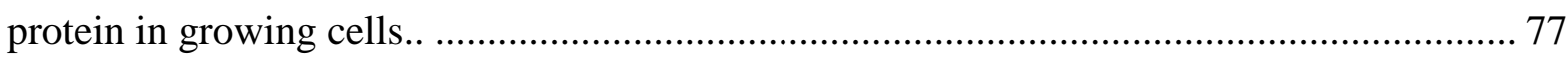

Figure 29: Successful recovery of purified Hat ${ }^{\mathrm{Tt}}-\mathrm{FZZ}$ protein detected by Western blot. ... 78 Figure 30: Indirect immunofluorescence of Hat $1^{\mathrm{Tt}}-\mathrm{FZZ}$ and untagged cells using anti-FLAG antibody 80

Figure 31: Multiple sequence alignments of human RBBP4/7, budding yeast Hat2 and $T$.

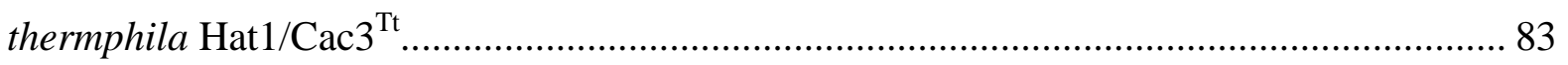

Figure 32: Structural comparison of various Hat2/Cac3Tt homologs............................... 84

Figure 33: Predicted tertiary structure of $T$. thermophila Hat $2 / \mathrm{Cac} 3^{\mathrm{Tt}}$ protein in comparison to

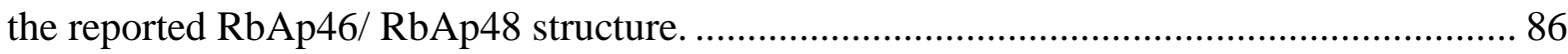


Figure 34: Western blot of Hat2/Cac $3^{\mathrm{Tt}}-\mathrm{FZZ}$ indicating the successful expression of the tagged protein in growing cells

Figure 35: Successful recovery of purified Hat2/Cac ${ }^{\mathrm{Tt}}$-FZZ protein detected by Western

blot.

Figure 36: Indirect immunofluorescence of Hat $2 / \mathrm{Cac} 3^{\mathrm{Tt}}-\mathrm{FZZ}$. Hat $2 / \mathrm{Cac} 3^{\mathrm{Tt}}$ localization is temporally correlated with mitosis in logarithmically growing T.thermophila (A-E)...... 93

Figure 37: Model for the transport of newly synthesized histones H3/H4 in T. thermophila. 98 Figure 38: Hierarchal clustering of gene expression data of T. thermophila putative HAT1, NRP1, ASF1, HIRA AND CAC2.. 109

Figure 39: Protein-protein interaction network based on AP-MS data and expression

profiles. 110

Figure 40: Hypothetical gene duplication resulting in division of functions.....

Figure 41: Updated version of $\mathrm{H} 3 / \mathrm{H} 4$ assembly pathway in $T$. thermophila.

Figure 42: Expression of Aip1-FZZ assessed by Western blot and IF analysis.

Figure 43: Sequence alignment indicating PIP in various Cac1 proteins. 115

Figure 44: Predicted CKII sites on Cac2 and Cac1 protein. 115 


\section{List of appendices}

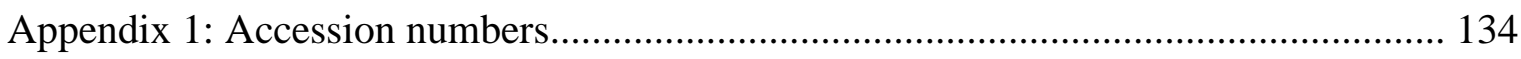

Appendix 2A: ClustalX default color legends.......................................................135

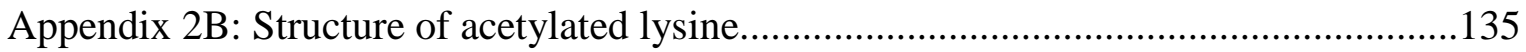

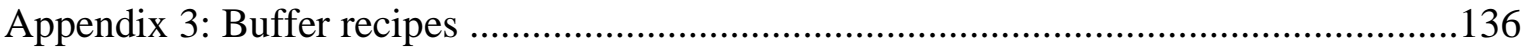

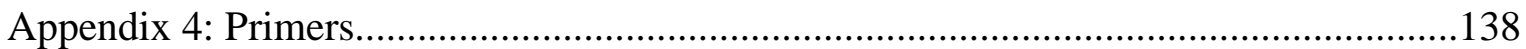

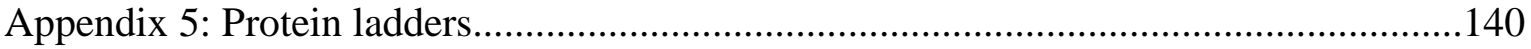




\section{List of abbreviations}

\begin{tabular}{|c|c|}
\hline Asf1 & Anti-silencing factor 1 \\
\hline$\AA$ & angstrom $(1.0 \times 10-10$ meters $)$ \\
\hline AP & affinity purification \\
\hline APS & ammonium persulfate \\
\hline BES & breakage eliminated site \\
\hline BLAST & Basic Local Alignment Search Tool \\
\hline bp & base pair \\
\hline Caf1 & Chromatin assembly factor 1 \\
\hline $\mathrm{C}-$ & Carboxy \\
\hline CBS & chromosome breakage site \\
\hline $\mathrm{ddH} 2 \mathrm{O}$ & double distilled water \\
\hline DMSO & Dimethyl sulfoxide \\
\hline DNA & Deoxyribonucleic acid \\
\hline EDTA & Ethylenediaminetetraacetic acid \\
\hline FZZ & 3x FLAG-ZZ epitope tag \\
\hline GFP & Green fluorescent protein \\
\hline Hat $1 / 2$ & Histone acetyl transferase $1 / 2$ \\
\hline Hira & Histone regulator $\mathrm{A}$ \\
\hline $\operatorname{IgG}$ & Immunoglobin $\mathrm{G}$ \\
\hline $\mathrm{Kb}$ & Kilo base pair \\
\hline $\mathrm{kDa}$ & kilodalton \\
\hline $\mathrm{KO}$ & Knockout \\
\hline $\mathrm{M}$ & molar \\
\hline
\end{tabular}




\begin{tabular}{|c|c|}
\hline MAC & macronucleus \\
\hline MIC & micronucleus \\
\hline MS/MS & tandem mass spectrometry \\
\hline$\mu$ & micro \\
\hline MW & molecular weight \\
\hline N- & amino \\
\hline NASP & Nuclear autoantigenic sperm protein \\
\hline NP-40 & nonidet $\mathrm{P}-40$ \\
\hline Nrp1 & NASP related protein 1 \\
\hline ORF & Open reading frame \\
\hline PAGE & Polyacrylamide gel electrophoresis \\
\hline PBS & Phosphate buffered saline \\
\hline PCR & Polymerase chain reaction \\
\hline PMSF & Phenylmethanesulfonylfluoride \\
\hline PSF & Penicillin streptomycin fungizone \\
\hline PTM & Post-translational modifications \\
\hline $\mathrm{RBBP} / \mathrm{RbAp}$ & Retino-blastoma binding/ associated protein \\
\hline $\mathrm{RD}$ & Replication dependent \\
\hline RI & Replication independent \\
\hline rpm & rotations per minute \\
\hline SAINT & Significance analysis of interactome \\
\hline scnRNA & scan RNA \\
\hline SDS & Sodium dodecyl sulfate \\
\hline snRNA & small nuclear RNA \\
\hline
\end{tabular}




$\begin{array}{ll}\text { SPP } & \text { Sequestrin proteose peptone } \\ \text { TAP } & \text { Tandem affinity purification } \\ \text { TBE } & \text { tris/borate/EDTA } \\ \text { TEMED } & \text { tetramethylethylenediamine } \\ \text { TEV } & \text { Tobacco etch virus } \\ \text { UTR } & \text { Untranslated region } \\ \text { UV } & \text { Ultraviolet } \\ \text { v/v } & \text { volume/volume } \\ \text { WCE } & \text { Whole-cell extract } \\ \text { WT } & \text { Wildtype } \\ \text { w/v } & \text { weight/volume } \\ \text { YPD } & \text { yeast extract peptone dextrose } \\ \text { YT } & \text { yeast extract tryptone } \\ \end{array}$


PART1: PROTEOMIC ANALYSIS OF MAJOR CHROMATIN ASSEMBLY

PROTEINS IN T. THERMOPHILA 


\section{Chapter 1: Introduction}

Eukaryotic genomic DNA is packaged into a nucleoprotein complex called chromatin. The fundamental repeating unit of chromatin is the nucleosome which consists of two of each of histones H2A, H2B, H3 and H4, wrapped by 147bp of DNA (Luger et al. 1997). Furthermore, one molecule of histone $\mathrm{H} 1$ or in some cases $\mathrm{H} 5$ binds to the linker region between two nucleosome particles and provides a higher level of compaction (reviewed in Bernas et al. 2014).

Changes in chromatin structure can influence all aspects of DNA-mediated cellular processes including replication, transcription, repair and recombination (reviewed in MariñoRamírez et al. 2005; Aalfs and Kingston 2000). To date three mechanisms have been identified to remodel chromatin structure. The first involves posttranslational covalent modifications (PTMs) of specific amino acids in the $\mathrm{N}$ - or $\mathrm{C}$-terminal tails of histones, e.g. acetylation, phosporylation, and ribosylation (Fischle et al. 2003) . The second mechanism is

the selective insertion of histone variants (reviewed in Biterge \& Schneider, 2014). For example, core histone $\mathrm{H} 3.1$ can be replaced with its variant $\mathrm{H} 3.3$ in a replication independent manner (Tagami et al. 2004). The third mechanism of chromatin remodelling occurs through physical disruption of nucleosomes mediated by ATP-dependent chromatin remodelling complexes such as SWI/SNF complex (Vignali et al. 2000).

\section{1- Chromatin assembly and Histone chaperones}

Chromatin assembly is the process of nucleosome formation (reviewed in Fischle et al. 2003). Nucleosome formation involves the deposition of one tetramer of histone H3 and H4, $(\mathrm{H} 3-\mathrm{H} 4)_{2}$, followed by the incorporation of two histone $\mathrm{H} 2 \mathrm{~A} / \mathrm{H} 2 \mathrm{~B}$ dimers onto DNA 
(Mariño-Ramírez et al. 2005). Studies have suggested that the process of chromatin assembly begins in the cytoplasm where the histones are synthesized (reviewed in Roth and Allis 1996). In particular deposition related histone H4 acetylation mediated by Histone acetyl transferase 1 (Hat1) occurs in the cytoplasm (Jackson et al. 1976; Sobel et al. 1995; Li et al. 2012). The N-terminal tails of newly synthesized H4 are diacetylated at lysine (K) residues 5 and 12 - a pattern of acetylation that has been found to be highly conserved in organisms ranging from protozoa to humans (Allis et al. 1985). Along the same lines, newly synthesized histones H3 have also been found to be highly acetylated, although with more variable patterns across different species (Sobel et al. 1995; Kuo et al. 1996). For example, H3K9, K14, K23 and K27 are the main sites of acetylation in Saccharomyces cerevisiae whereas in Tetrahymena thromphila K9 and K14 are preferentially acetylated (Kuo et al. 1996). These covalent modifications have been suggested to be important for chromatin assembly.

Transport of newly synthesized histones from the cytoplasm to the nucleus is mediated by a diverse set of proteins including chaperones and karyopherins (Kaps)(Keck and Pemberton 2012) . The transport mechanism of H2A/H2B is not fully understood, however histone chaperone 'Nucleosome assembly protein 1(Nap1)' along with a Kap114 has been shown to be key player in this process (reviewed in Keck and Pemberton 2012). The transport of $\mathrm{H} 3 / \mathrm{H} 4$ has been subject of intense research and numerous histone chaperones have been shown to be involved in this process. In humans, current models suggest that newly synthesized histones $\mathrm{H} 3.1 / \mathrm{H} 4$ are shuttled through at least four different cytosolic complexes (Campos et al. 2010; Alvarez et al. 2011). The process begins with the interaction of H3.1-H4 with Heat shock proteins (HSP90) that possibly aid in the proper folding of newly synthesized histones (Campos et al. 2010). Subsequently, H3.1-H4 are passed to a 
histone chaperone called Nuclear Auto-antigenic Sperm Protein (NASP) which assists in Hat1/Hat2 dependent acetylation of newly synthesized histone H4. Finally histones H3.1-H4 are transferred to Anti-silencing factor 1 (Asf1) that interacts with importin4 and transfers them to the nucleus (See Figure 1) (Campos et al. 2010).

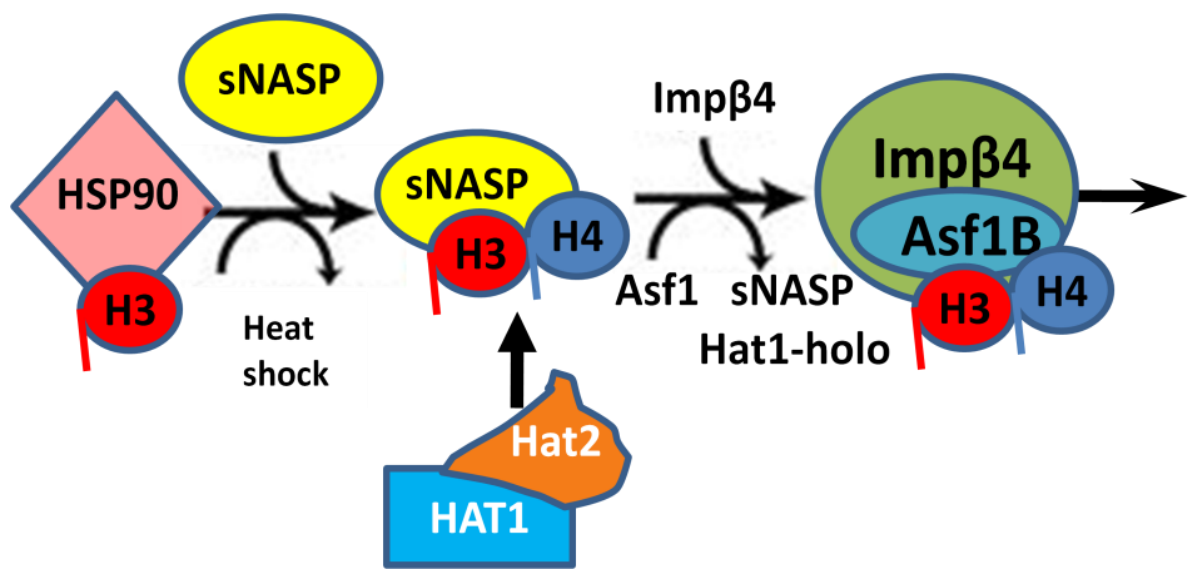

Figure 1: Schematic representation cytoplasmic of events during the transport of histones $\mathbf{H 3} / \mathbf{H} 4$ to the nucleus. Histones $\mathrm{H} 3 / \mathrm{H} 4$ are shuttled through various protein complexes in a stepwise fashion before entering the nucleus (see text for further details) (Image is designed after Campos et al. 2010)

Additionally, Codanin-1 has been shown to negatively regulate the supply of these histones to the nucleus by sequestering Asf1 via a physical interaction in the cytoplasm (Ask et al. 2012). Once inside the nucleus, cells can adopt one of two pathways to deposit the histones onto the DNA (See Figure 2). The first process is called replication dependent (RD) chromatin assembly which occurs concurrently with DNA replication or repair (Smith and Stillman 1989; Kaufman et al. 1995). RD chromatin assembly is mediated by a three subunit protein complex called 'Chromatin Assembly Factor 1 (CAF1)' that physically interacts with Asf1 (Tagami et al. 2004) to obtain histones H3.1-H4 before subsequently depositing them onto the replicating DNA through its physical interaction with 'Proliferating cell nuclear antigen (PCNA)' (Kaufman et al. 1995; Ramirez-Parra and Gutierrez 2007; Rolef BenShahar et al. 2009). CAF1 complex consists of three subunits, namely chromatin assembly complex (Cac) proteins Cac1, Cac2 and Cac3, and respectively called as p150, p60 and p48 
(also RbAp48 or RBBP4) in humans. Among these subunits, Asf1 physically interacts with Cac2 to pass the histones H3.1-H4 to the CAF1 complex (See Figure 2) (Malay et al. 2008).

Replication independent (RI) chromatin assembly occurs outside of the S-phase (Green et al. 2005). It is mediated by 'Histone regulatory homolog A (Hira)' that interacts with Asf1 through its B-domain in a mutually exclusive manner with the Cac 2 subunit of CAF1 (Sherwood et al. 1993; Tang et al. 2006). RI assembly involves replacement of core histones with histone variants and Hira primarily is responsible for depositing histone $\mathrm{H} 3$ variant $\mathrm{H} 3.3$ (See Figure 2) (Tagami et al. 2004; Jullien et al. 2012). In budding yeast which lacks H3.3, the Hira complex (see below) along with Asf1 deposits H3/H4 via RI pathway suggesting that Hira proteins functions are conserved among eukaryotes (Green et al. 2005).

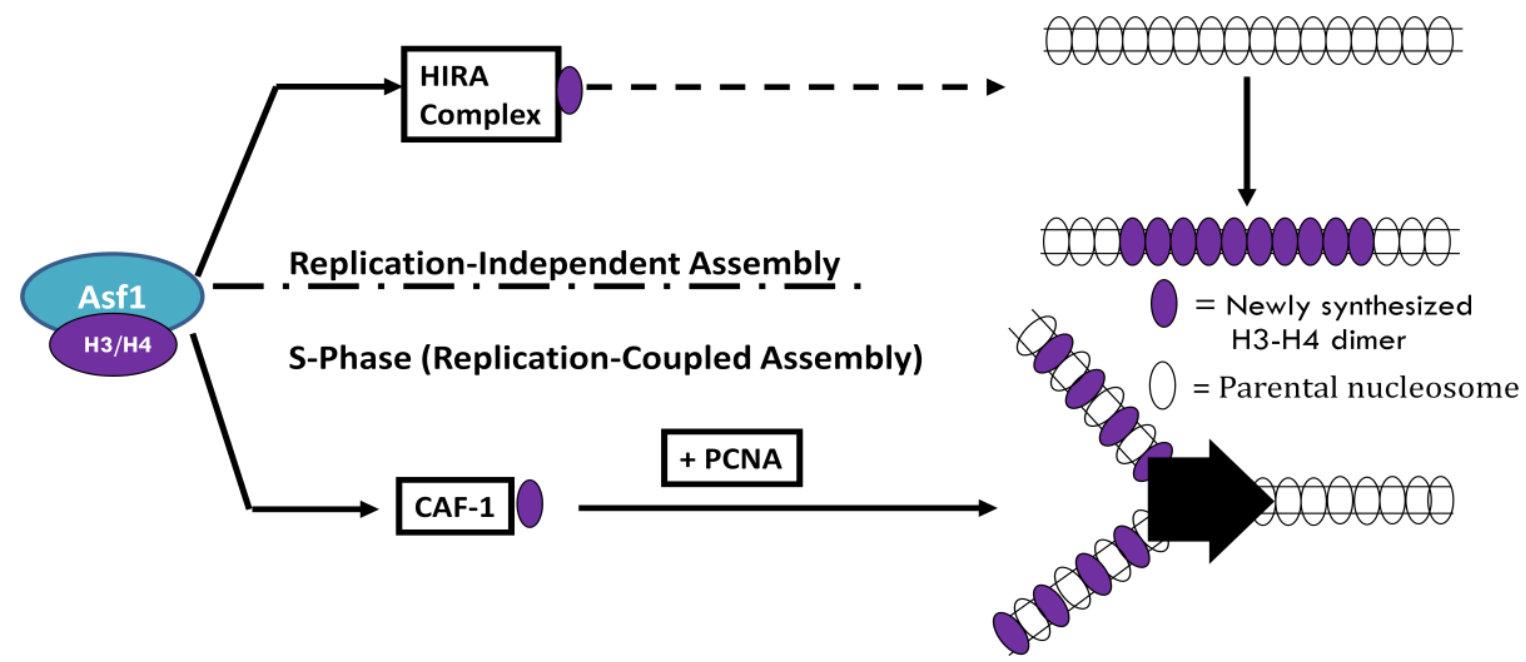

Figure 2: Chromatin assembly pathways. Asf 1 transfers newly synthesized histones H3/H4 either to HIR complex for replication independent chromatin assembly or to CAF1 complex for replication coupled assembly. Thick black arrow represents replication fork (Image adopted from Fillingham and Greenblatt 2008)

\section{2- Histone chaperones}

Histone chaperones, by definition, are the proteins that can bind with free histones, shield their positive charge, and subsequently deposit them onto DNA in an orderly manner without themselves being a part of the chromatin (De Koning et al. 2007). Histone 
chaperones can be grouped into several families based on their overall structural/sequence similarities as well as binding specificities (De Koning et al. 2007). For example, Asf1 and CAF1 are categorized as $\mathrm{H} 3 / \mathrm{H} 4$ chaperones whereas Nap1 is chiefly H2A/H2B chaperone.

\subsection{2- Anti silencing factor 1 (Asf1)}

Asf1 is a highly conserved histone $\mathrm{H} 3 / \mathrm{H} 4$ chaperone that has been found in all examined eukaryotes suggesting that it most likely was present in the last eukaryotic common ancestor (Balaji et al. 2009). It was first isolated as a gene whose over-expression led to the derepression of the yeast silent mating type locus (Le et al. 1997). Asf1 has a central role in both RD and RI chromatin assembly pathways through its mutually exclusive interactions with CAF1 and Hira, respectively, to transfer either $\mathrm{H} 3 / \mathrm{H} 4$ or $\mathrm{H} 3.3 / \mathrm{H} 4$ (for review Bao and Shen 2006; Malay et al. 2008; Yamane et al. 2011). In vertebrates such as humans, Asf1 exists as two paralogs Asfla and Asf1b. Recently, it was shown that these paralogs have experienced an event of subfunctionalization with Asf1a acquiring high binding affinity for Hira whereas Asf1b preferentially binds with CAF1(Abascal et al. 2013).

Asf1 is a functionally diverse protein with several important roles in various cellular processes such transcription activation and repression (Adkins et al. 2007; Fillingham et al. 2009), histone transport (Campos et al. 2010) and chromatin assembly (Green et al. 2005), maintenance of genomic stability (Prado et al. 2004), DNA replication and repair (Schulz and Tyler 2006) and exit from DNA damage checkpoint (Emili et al. 2001). In budding yeast $S$. cerevisiae Asf1 also functions to promote $\mathrm{H} 3 \mathrm{~K} 56$ acetylation mediated by a fungal specific HAT Rtt109 by presenting H3/H4 to it (Han et al. 2007). Similarly, in humans Asf1 promotes $\mathrm{H} 3 \mathrm{~K} 56 \mathrm{ac}$ by its interaction with CBP (Creb-binding protein) HAT (Groth et al. 2007). This acetylation has been shown to be important in conferring to the cell resistance to the DNA damaging agents (Masumoto et al. 2005). Proteomic analysis of Asf1 in budding 
yeast has revealed several physical interactions including Hira-complex, the nuclear Hat1 complex and Rad53 check point kinase in addition to H3.1/H4 (Emili et al. 2001; Green et al. 2005; Prochasson et al. 2005; Fillingham et al. 2008). Furthermore, an epitope tagged human Asfla co-purifies with several proteins including Hira, CAF1, Importin4, NASP, Codanin-1, RbAp46/48, MCM2-7 replicative helicase and histone H4 (Tagami et al. 2004; Groth et al. 2007; Ask et al. 2012). These interactions are consistent with the above described functions of Asf1 in histone transport and chromatin assembly pathways.

Recently, we showed that $T$. thermophila homolog of Asf1 co-purifies with a NASP family protein Nrp1 (NASP-related protein 1), an importin $\beta$ (ImpB3), a RING-domain containing protein that also has C-terminal BRCT repeats similar to those of human BRCA1 and two novel proteins that we have named Asf1-interacting protein (AIP)1 and AIP2 (Garg et al. 2013). These observations suggested that Asf1 has an ancient role in the transport of histones $\mathrm{H} 3 / \mathrm{H} 4$.

\subsection{3- Nuclear autoantigenic sperm protein (NASP)}

Initially discovered in Xenopus laevis oocytes, N1/N2 is the founding member of NASP family proteins and was found to specifically bind H3-H4 providing a mechanism of the storage of soluble histones required for DNA replication in early embryo (Kleinschmidt et al. 1985; Dilworth et al. 1987). NASP, which is the mammalian homolog of N1/N2, was first identified in rabbit testes as a highly autoantigenic protein (Welch et al. 1990). Subsequently, it was shown that in mammals NASP predominantly exists as two alternatively spliced isoforms (Richardson et al. 2000). The full length NASP (tNASP) is primarily expressed in embryonic tissues and testes whereas the smaller spliced version called somatic NASP (sNASP) lacking a region of 339 amino acids is ubiquitously expressed in all dividing cells (Richardson et al. 2000). Subsequent studies revealed that NASP is essential for mammals 
and its deletion results in an early embryonic lethality in mice (Richardson et al. 2006). Furthermore, NASP expression has been shown to be tightly cell cycle regulated (Alekseev et al. 2003).

In humans, NASP has been shown to participate in diverse cellular processes including histone $\mathrm{H} 3 / \mathrm{H} 4$ transport, linker histone $\mathrm{H} 1$ chaperone activity and Hat1-dependent acetylation of H4 (Finn et al. 2008; Campos et al. 2010). Recently it was shown that human NASP also functions in the maintenance of soluble reservoirs of histones $\mathrm{H} 3 / \mathrm{H} 4$ through chaperone mediated autophagy (Cook et al. 2011). NASP family proteins share conserved motif architecture of four tetratricopeptide repeats (TPR) where the second TPR is generally interrupted by large acidic patch (Dunleavy et al. 2007). The TPR motifs are 34 amino acid long amphipathic helices that form a helix-turn-helix arrangement (D'Andrea and Regan 2003). These motifs are thought to mediate protein-protein interactions by providing a structure scaffold for the assembly of biological molecules (D'Andrea and Regan 2003). Different TPRs in human sNASP has been shown to carry out distinct functions (Wang et al. 2012). For example, in vivo as well as in vitro data has indicated that TPR2 interacts with hitone H1 whereas TPRs 3/4 participate in H3/H4 interactions (Wang et al. 2008; Wang et al. 2012).

NASP family proteins have been shown to exhibit some functional diversity among different species. For example, the NASP homolog in S. cerevisiae is called Hif1 (Hat1 interaction factor 1) and functions in the acetylation of histone $\mathrm{H} 4$ through physical interactions with Hat1 and Hat2 proteins (Ai and Parthun 2004; Poveda et al. 2004) whereas Schizosaccharomyces pombe NASP homolog called as Sim3 (Silencing in the middle of the centromere protein 3) specifically functions in the deposition of centromeric $\mathrm{H} 3$ variant and does not interact with Hat1-complex (Dunleavy et al. 2007). Despite the demonstrated roles 
of NASP in various cellular processes, the underlying mechanistic details are still unclear. For example, whether NASP is also involved in nucleosome formation or does it only maintain soluble histone.

\subsection{4- Histone Regulatory Homolog A (HIRA)}

The Hira complex in S. cerevisiae is composed of four subunits including Hir1, Hir2, Hir3 and HPC2 (histone promoter control 2) and was initially described as a repressor of three histone gene loci (Osley and Lycan 1987; Kaufman et al. 1998; Green et al. 2005). The Hir1 and Hir2 as well as Slm9 and Hip1 proteins are lineage-specific paralogs in $S$. cerevisiae and $S$. pombe, respectively, and are both homologs to the single Hira protein in humans (Blackwell et al. 2004; Greenall et al. 2006). Recent studies using database searches have identified human CABIN1 and UBN1 as the orthologs of Hir3 and HPC2, respectively (Balaji et al. 2009). These studies indicated that Hira complex is evolutionarily conserved and most likely evolved with the emergence of eukaryotes. Consistently, accumulating biochemical evidence has established that CABIN1 and UBN1 co-purify with human Hira (Rai et al. 2011; Tang et al. 2012). Hira is a WD40 repeat containing protein and structural analysis has revealed that human Hira has at least seven such repeats (reviewd in Song et al. 2013). These WD40 repeats assume a seven bladed beta propeller structure which is thought to provide a rigid structural scaffold for mediating protein-protein interactions (Balaji et al. 2009; Xu and Min 2011).

Hira complex in yeast has been shown to have several functions including transcription repression and heterochromatin assembly (Kaufman et al. 1998; Sharp et al. 2001; Blackwell et al. 2004). In budding yeast, mutations in HIR genes are known to display synthetic defects

or lethality when combined with mutations in genes that encode the components of transcription elongation factor (FACT) complex (Formosa et al. 2002). This observation 
suggests a functional importance of Hira complex in transcription regulation. In addition, Hira complex plays major role in transcription repression by interaction with nucleosomes which in turn prevents chromatin remodelling activity of SWI/SNF complex (Prochasson et al. 2005). In fission yeast, loss of members of Hira complex results in silencing defects at the centromere and mating type loci (Blackwell et al. 2004; Greenall et al. 2006).

Interestingly, Hira has also been shown to function as the positive regulator of transcription. For example, chicken Hira protein can positively and negatively regulate different sets of cell-cycle related genes through its $\mathrm{N}$-terminal and C-terminal halves, respectively (Ahmad et al. 2005). Furthermore, in higher eukaryotes including humans, Hira functions in RI chromatin assembly pathway and deposits histone H3 variants including H3.3 and H3.2 onto the DNA (Tagami et al. 2004; Green et al. 2005; Latreille et al. 2014). Hira in humans and Hir1 in budding yeast interact with Asf1 through a region called B-domain, which also is present in Cac2 subunit of CAF1 complex, to acquire newly synthesized histones (Tang et al. 2006) that are then deposited onto DNA in RI manner (Green et al. 2005). Replacement of H3 with H3.3 can alter the biochemical properties of the chromatin by providing post-translational modification sites distinct from those of $\mathrm{H} 3$ (reviewed in Biterge and Schneider 2014). Several studies have shown that H3.3 is generally deposited in euchromatin (Ahmad and Henikoff 2002) and it is enriched in covalent modifications that are linked to transcriptionally active genes (McKittrick et al. 2004).

\subsection{5- Chromatin Assembly Factor 1 (CAF1)}

CAF1 was originally identified as a factor that functions in the chromatin assembly during Simian virus40 origin dependent DNA replication in human cell extracts (Smith and Stillman 1989). Subsequently it was shown that human CAF1 consists of three subunits including p150, p60 and p48 (Kaufman et al. 1995) which in budding yeast are respectively 
called as Cac1, Cac2 and Cac3. A recent study identified CAF1 components throughout the major eukaryotic super-groups suggesting that CAF1 is a highly conserved protein complex (Balaji et al. 2009).

CAF1 has a critical function to deposit newly synthesized histones $\mathrm{H} 3 / \mathrm{H} 4$ via $\mathrm{RD}$ chromatin assembly pathway (Verreault et al. 1996; Hoek and Stillman 2003). The p150/Cac1 largest subunit of CAF1 physically interacts with PCNA to deposit newly synthesized histones H3/H4 (Shibahara and Stillman 1999). There are two PCNA binding motifs (PIP1 and PIP2) on p150/Cac1 however CAF1 localization to the replication forks occurs through PIP2 that resides in the middle of the p150 open reading frame (Rolef BenShahar et al. 2009). The interaction between CAF1 and PCNA has been demonstrated by coimmunoprecipitation and glutathione-S transferase (GST) pull-down experiments (Shibahara and Stillman 1999; Moggs et al. 2000). Interaction with PCNA is consistent with the reported functions of CAF1 in RD, as well as DNA repair associated chromatin assembly.

Similar to its paralog Hira, the p60 subunit of CAF1 largely consists of WD40 repeats and displays H3/H4 binding activity (Balaji et al. 2009). This subunit contains two Bdomains like regions which are found outside of the WD40 repeats and mediate CAF1 interaction with Asf1(Tang et al. 2006). Interestingly, CAF1 and Hira interact with Asf1 in a mutually exclusively manner indicating a division of RD and RI chromatin assembly pathways, respectively (Tang et al. 2006; Yamane et al. 2011).

The p48 subunit of CAF1 is also known as retinoblastoma binding protein RBBP4/ RBAP48 has been found in several H3-H4 binding complexes including histone deacetylase (HDAC) complex (Murzina et al. 2008). The p48 directly binds with histone H4 through the helix 1 of the histone fold, a region that is not accessible when H4 is in chromatin (Murzina 
et al. 2008; Song et al. 2008). Interestingly, p48 is highly homologous to RBBP7/ RBAP48 (also known as Hat2 in yeast) (Murzina et al. 2008) which is a subunit of Hat 1 complex responsible for the diacetylation of newly synthesized histones H4 (reviewed in Parthun 2012).

In budding yeast cells lacking $C A C 1, C A C 2$ and $C A C 3$ are viable however show sensitivity to a variety of DNA damaging agents, including bleomycin, methane methylsulfonate (MMS), and UV radiation (Kaufman et al. 1997; Linger and Tyler 2005). These studies are consistent with role of CAF1 in DNA repair associated chromatin assembly. Furthermore, CAF1 has also been implicated in the epigenetic inheritance, most likely through the maintenance of histone density during DNA replication (reviewed in Ridgway and Almouzni 2000). In budding yeast CAF1 has been shown to interact with Sas1 (Something about silencing) which has a critical role in DNA replication fork progression (Zhong et al. 2013). In humans and mouse CAF1 has been shown to interact with heterochromatin protein 1 (HP1) and methyl-DNA-binding protein MBD1, respectively (Murzina et al. 1999; Reese et al. 2003) suggesting a role in the formation of proper heterochromatin structure (Quivy et al. 2008).

Despite the identification of numerous functions of CAF1 in the context of multiple complexes, questions remain about the underlying mechanistic details. For example, it has been suggested that CAF1 functions in the epigenetic inheritance by recruiting various chromatin remodelling complexes(reviewed in Ridgway and Almouzni 2000) however the exact mechanism is poorly understood. In addition, CAF1 has been shown to exist in a phosphorylated form but the functional significance and the identity of the kinase involved is unclear (Martini et al. 1998). 


\section{3- Histone posttranslation modifications}

As noted earlier, one method of chromatin remodelling is through histone PTMs, including acetylation, phosphorylation, methylation (mono, di and trimethylation), sumoylation and ubiquitylation (Fischle et al. 2003). These covalent modifications can both be rapidly added and removed from the histones depending upon the different phases of cell cycle and in response to changes in environmental conditions (for review Bannister and Kouzarides 2011). Alternatively, they can be longer lasting in the form of heritable epigenetic marks (Zhu and Reinberg 2011; Keck and Pemberton 2012). Importantly, some of these epigenetic marks result in a particular biological outcome. For instance, the trimethylation of H3K9 in metazoans provides a signal for the recruitment of HP1 and other proteins that facilitate gene silencing (Horn and Peterson 2006). One important histone PTM related to chromatin assembly is the diacetylation of $\mathrm{H} 4$ at the $\mathrm{N}$-terminal tail. The diacetylation of $\mathrm{H} 4 \mathrm{~K} 5 / 12$ is highly evolutionary conserved raising the possibility that this PTM is significantly relevant to the process of chromatin assembly (Allis et al. 1985; Parthun 2012).

\subsection{2- Histone acetyl transferase1 (HAT1)}

HATs are the enzymes responsible for the transfer of an acetyl moiety from acetyl coenzyme A onto the $\varepsilon$-amino group of one or more target lysine residues on the histones (reviewed in Parthun 2012). Based on their structurally conserved core including the binding sites of acetyl CoA, HATs have been grouped under the big umbrella of GNAT super-family of acetyl transferases (Vetting et al. 2005). Traditionally, HATs have been classified into two classes, i.e. type A and type B HATs. The type-A HAT enzymes such as GCN5 are 
primarily nuclear whereas type B HATs are cytoplasmic and acetylate free histones (mainly H4) prior to their deposition onto chromatin (reviewed in Parthun 2007).

Hat1 is the founding member of type B HATs and was initially thought to be primarily cytoplasmic (Parthun et al. 1996). Subsequent biochemical characterization however revealed that although it does localize in the cytoplasm but it predominantly is a nuclear enzyme (Ruiz-García et al. 1998). Hat1 structure has been resolved and is highly conserved from yeast to humans (Dutnall et al. 1998; Li et al. 2014). The core Hat1 complex which originally was isolated from the yeast cytoplasmic extracts consisted of two subunits, i.e. the catalytic Hat1 and a WD40 repeat protein called Hat2 (RBBP7/ RBAP46) (Ruiz-García et al. 1998). Hat2 directly binds with histone $\mathrm{H} 4$ and is required for the catalytic activity Hat 1 (Ruiz-García et al. 1998; Ai and Parthun 2004). Subsequently, it was shown that Hat1complex has an additional nuclear subunit called Hif1 which is a member of NASP family proteins and interacts with Hatl complex at roughly stoichiometric levels (Poveda et al. 2004; Fillingham et al. 2008) . The nuclear Hat1 complex consisting of Hat1-Hat2-Hif1 is known as NuB4 (Nuclear type B HAT specific for H4) (reviewed in Parthun 2012). Interestingly, recent evidence both from yeast and humans suggests that interaction between Hat1 core complex and a NASP family protein (such as Hif1) may also occur in the cytoplasm suggesting an important role for this complex in the transport of $\mathrm{H} 3 / \mathrm{H} 4$ to the nucleus (Campos et al. 2010; Alvarez et al. 2011).

Newly synthesized histones $\mathrm{H} 4$ are diacetylated at K5 and K12 positions (H4K5ac and H4K12ac) at the NH2-termini. Subsequently, histones H3 then join the complex which then is shuttled to the nucleus. The pattern of H4K5ac and H4K12ac has been shown to be conserved but a specific function for these marks remains unclear. These modifications have been associated with the chromatin assembly however several studies indicate that they only 
have a minor role in this process. For instance, genetic studies in yeast which involved the mutations of K5 and K12 to arginine in $\mathrm{H} 4$ showed little effect on chromatin assembly or cell viability (Ma et al. 1998; Ge et al. 2013).

Recent evidence suggests that in addition to acetylate newly synthesized H4, Hat1 is also responsible to maintain the acetylation of histone $\mathrm{H} 3$ on $\mathrm{K} 9,18$, and 27 during RD chromatin assembly and is essential in mammals for normal development and genome stability (Ge et al. 2013; Nagarajan et al. 2013). Furthermore, NuB4 complex is important for the DNA repair through homologous recombination by facilitating the histone turnover (Yang et al. 2013). The NuB4 complex (Hat1-Hat2-Hif1) also interacts with Asf1 which has a role in various chromatin assembly pathways as noted earlier. The interaction between Asf1 and NuB4 that has been detected through affinity purifications is evolutionary conserved and has been reported in yeast, humans, and chicken cells (Barman et al. 2008; Fillingham et al. 2008; Campos et al. 2010). However, it is important to note that NuB4-Asf1 interaction requires the presence of Hat 2 and Hif1 subunits suggesting that it is mediated by histones H3/H4. Furthermore, yeast TAP-tagged Hat1 also co-purifies with all six subunits of origin recognition complex (ORC) complex in addition to Hat2 and Hif1 (Suter et al. 2007). Taken together, these studies suggest that Hat1 is a central protein for various cellular processes particularly deposition related $\mathrm{H} 4$ acetylation and DNA repair.

\section{4- What is Tetrahymena thermophila}

The eukaryote Tetrahymena thermophila is a single-celled organism that belongs to the phylum Ciliophora and can be found in freshwater systems (Orias et al. 2011). T. thermophila has been named as such due to the fact that it has four (tetra) membrane-like (hymen) oral structures (Lynn and Doerder 2012). Furthermore, as apparent from the name it 
can survive at high temperatures in comparison to the other ciliates. Although the preferred temperature for optimal growth is $30^{\circ} \mathrm{C}$, however $T$. thermophila has been found to survive in a temperature range of $12^{\circ} \mathrm{C}$ to $42^{\circ} \mathrm{C}$.

From an evolutionary view point ciliates diverged quite early during the eukaryotic evolution. They together with dinoflagellates, apicomplexans, oomycetes, diatoms and brown algae belong to the kingdom chromalveolata (Adl et al. 2012). Thus T.thermophila is distantly related to humans; however it has 2,280 human ortholog genes (Eisen et al. 2006) making it a suitable model organism to study human biology and gene function evolution.

\subsection{2-Nuclear dimorphism}

Ciliates share a common feature of exhibiting nuclear dimorphism in form of two spatially and structurally distinct nuclei, the micronucleus (MIC) and macronucleus (MAC) (See Figure 3). T. thermophila MAC genome has been sequenced and it contains $\sim 104 \mathrm{Mb}$ of DNA with roughly 25000 known or predicted protein coding genes (Eisen et al. 2006). MAC - the larger nucleus, is polyploid with $\sim 45$ copies of most genes and $\sim 9000$ copies of rDNA (reviewed in Orias et al. 2011). The MAC contains 180 acentromeric chromosomes of varying sizes that range from $\sim 21 \mathrm{~kb}$ to greater than $3000 \mathrm{~kb}$ (Cervantes et al. 2006; reviewed in Orias et al. 2011). On the other hand, the MAC rDNA chromosome is only about $\sim 21 \mathrm{~kb}$. It is palindromic and contains two copies of rDNA genes (reviewed in Orias et al. 2011). The MAC is highly evolved gene expression machinery and all known gene expression during $T$. thermophila vegetative growth takes place from here. It is sexually not inherited and therefore often is termed as somatic nucleus (reviewed in Orias et al. 2011).

Smaller of the two nuclei-the MIC is a diploid nucleus that remains transcriptionally silent during vegetative growth and is responsible for the faithful transmission of the genome to the progeny. It contains five metacentric chromosomes and divides mitotically during 
vegetative growth (reviewed in Orias et al. 2011). MIC genome encompasses a total of $\sim 120 \mathrm{Mb}$ sequence indicating that it has $\sim 15 \%$ greater DNA complexity than MAC. The difference between two genomes has been explained in terms of "internally eliminated sequences" (IESs), which are deleted in a site-specific manner during MAC differentiation (Yao et al. 1984).

Figure 3: Vegetative $T$. thermophila stained with DAP1 to demonstrated two nuclei. The bigger one is macronucleus whereas the smaller one that sits in recess is micronucleus.

\subsection{3- Life cycle}

As is the case with other ciliates, the life cycle of $T$. thermophila has two major phases, the vegetative growth and the sexual phase called conjugation.

\subsection{4- Vegetative growth}

During vegetative growth $T$. thermophila reproduce asexually through binary fission (reviewed in Orias et al. 2011). The MIC divides through mitosis providing the identical genetic content to the two daughter cells. Immediately after the conjugation (see below), the cells are considered immature as they lack the ability to conjugate. After $\sim 100$ vegetative divisions, cells become mature when they are capable of mating and conjugating (for review Orias et al. 2011). However, during vegetative growth as cells keep dividing they reach to a point of infertility at which they are no longer are capable of mating. The number of doublings that takes to reach infertility is highly variable (Pitts and Doerder 1988). Interestingly, as there is no gene expression taking place from the MIC it is not under any 
selective pressures against the loss of chromosomes. As a result, cells can continue to grow indefinitely and are considered virtually immortal. This immortality is achieved because MAC has active telomerase that prevents against telomeric loss of chromosomes (Greider and Blackburn 1987).

On the other hand, MAC divides by amitosis and lacks a mechanism of equal segregation of alleles. As a result, the genetic material is randomly distributed to the daughter nuclei. This phenomenon has provided the foundation for phenotypic assortments - a process through which a heterozygote can become homozygous for a particular gene (Orias; Orias et al. 2011). The process of phenotypic assortment can be accelerated through selection and generally takes approximately 62 vegetative generations (Figure 4)

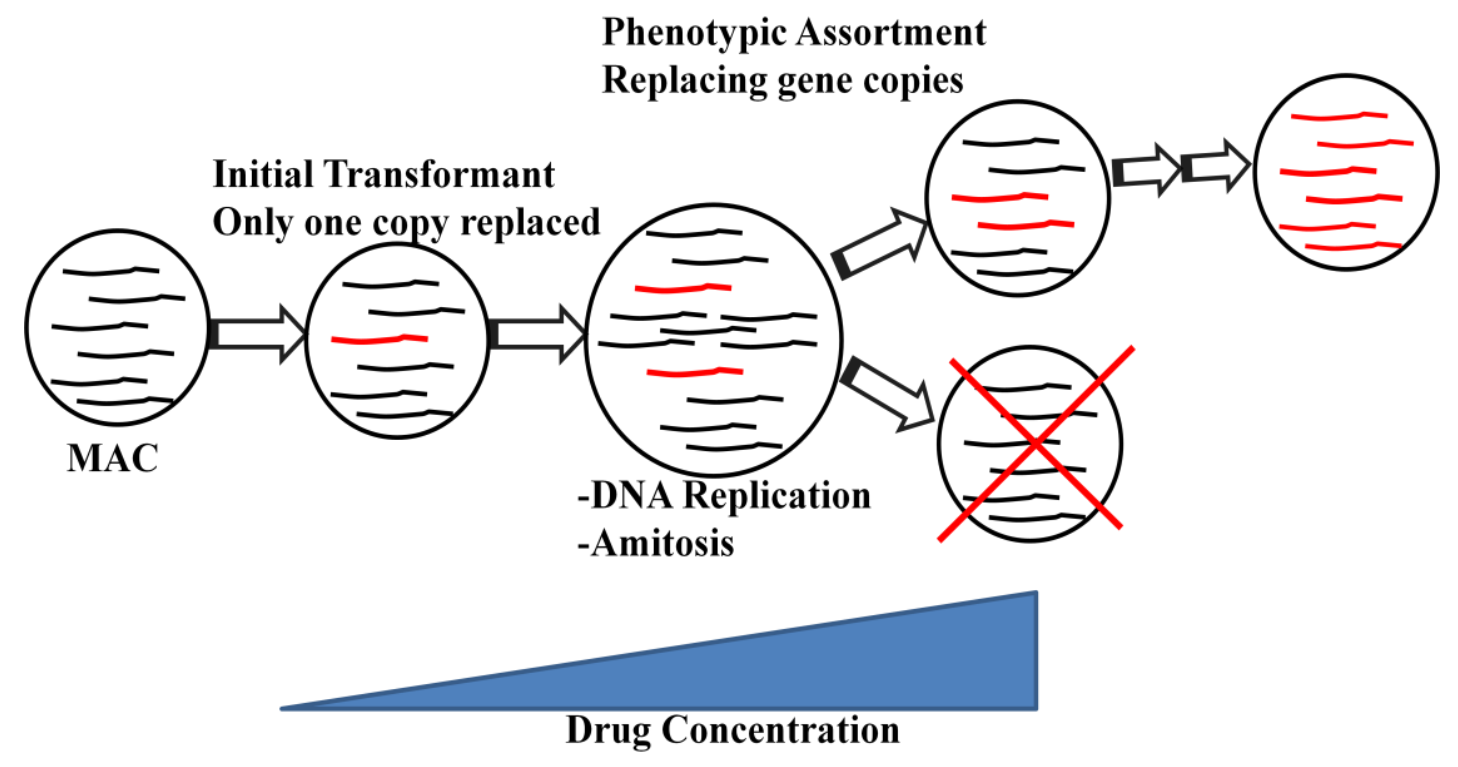

Figure 4: Schematic representation of accelerated phenotypic assortment. The macronucleus divides by amitosis and does not have a method for the equal segregation of alleles. Gradual increase of selective pressure by increasing the drug concentration results in homozygosity for a particular allele in the macronucleus.

\subsection{5- Conjugation}

The sexual phase of $T$. thermophila is called conjugation and it does not involve cell reproduction. T. thermophila has seven mating types (I to VII) and each mating type is 
capable of mating with the other mating type but not with the cells of same mating type (Martindale et al. 1982). Mating is inducible in laboratory settings when cells of opposite mating types are starved for $\sim 18$ hours and subsequently mixed in roughly equal numbers.

During conjugation, cells of two opposite mating types come close together and fuse their oral apparatuses. Initially, MIC elongates and adopts a shape commonly termed as 'crescent shape" which marks the beginning of meiosis. The cells then complete meiosis I and meiosis II reductional divisions and four haploid nuclei are produced. Three out of these nuclei are degraded leaving one which remains in the anterior of the cell. This anterior gametic nucleus then undergoes mitosis resulting in two identical nuclei celled pro-nuclei. One of these nuclei remains in the anterior and is called the migratory nucleus whereas the other one called as stationary nucleus is found in the posterior of the cell. The migratory pro-nuclei of the fused cells are exchanged and subsequently exchanged migratory nuclei are fused with the stationary nuclei within each mating partner. The resulting nucleus in each mating partner thus becomes diploid and is called as 'zygote nucleus'. The zygote nucleus undergoes two mitotic divisions, giving rise to 4 genetically identical diploid nuclei. The two anterior nuclei differentiate into macronuclei, while posterior products remain as diploid micronuclei. This is the stage at which site-specific DNA rearrangements and mating type determination occurs in the MAC. As the daughter MACs start developing - a stage referred as anlagen 1, the parental MAC moves toward the posterior end of the cell where it begins to getting degraded via a process analogous to lysosomal autophagy (Akematsu and Endoh 2010). As a result, gene expression switches from the parental to the developing MAC (zygotic MAC). Subsequently, cells enter the anlagen II stage where the developing MACs align in the center with a perpendicular orientation with two MICs on each side. The parental MAC keeps getting degraded until after the cells have separated (called as ex-conjugants). The old MAC 
and one of the two new MICs are destroyed in ex-conjugants. Subsequently, ex-conjugants undergo the first postzygotic cell division upon introducing the food source which results in the 4 karyonide cells (Martindale et al. 1982).

\subsection{6- Programmed DNA rearrangements}

During conjugation, the conversion into MAC involves major programmed DNA rearrangements. These rearrangements result in the extensive removal of DNA $(\sim 15 \%)$ from the developing anlagen and the remaining genome is endo-replicated $\sim 50$ times (Yao et al. 1984). An extensive amount of literature exists regarding the process of DNA rearrangements in $T$. thermophila and only a brief overview is provided below.

Two types of genome rearrangements have been documented in $T$. thermophila. The first one is the deletion of sequences from the MIC called internal eliminated sequences (IESs) which are $~ 6000$ in number and vary in length from 0.5 to $20 \mathrm{~Kb}$ (Yao et al. 1984). DNA elimination in $T$. thermophila occurs reproducibly at a specific site or at a limited number of alternative sites. The boundaries of IESs have been found to be relatively precise, however no consensus sequence motif have been found in or around IESs (Austerberry et al. 1989). Some cis-acting sequences have also been identified that flank the IES and are necessary for its removal (Godiska et al. 1993). However no sequence homology has been observed across different elements.

It has been proposed that DNA removal acts like a defence mechanism against viruses and transposans that might produce detrimental consequence when present inside the MAC. In a study Yao and Fuller (2003) created heterokaryons with a bacterial transposon Tn5 neomycin resistance gene. They introduced this sequence at several sites within the MIC but not in the Mac. This element was eliminated from the anlagen when the cells were 
conjugated forcing a new round of MAC development (Yao et al. 2003). Thus DNA removal from anlagen is an active system of defence against invading parasitic DNA. Furthermore, the non-coding repeat nature of IESs resembles closely with the transposans and heterochromatic regions in other eukaryotes. These observations also suggest that $T$. thermophila has evolved one of the most extreme methods of gene silencing in which sequences are 'silenced' by permanent removal from the transcriptionally active MAC genome (Mochizuki et al. 2002).

IESs elimination has been found to occur via an RNAi like mechanism and a scan RNA model has been proposed (Mochizuki et al. 2002). According to this model the MIC chromosomes are transcribed in both directions during prophase of meiosis to produce dsRNAs which are then cleaved into smaller RNAs (scnRNAs) of 27-30 nucleotides in length through the action of the Dcl1 "Dicer-like" protein (Mochizuki and Gorovsky 2005). The scnRNAs form a complex with a Piwi protein homolog, Twi1 and are then transported to the parental MAC by Giw1 (Gentleman in waiting I) (Mochizuki and Gorovsky 2004; Noto et al. 2010). The Twil protein then interacts with an RNA helicase Ema1 to conduct a homology search for the parental MAC ncRNAs. Subsequently, those scnRNAs that match with ncRNAs are degraded (Mochizuki et al. 2002). The remaining scnRNAs in a complex with Twil and Ema1 are transported back to the anlagen where another homology search is carried out (Aronica et al. 2008). Any sequences that are found homologous to the scnRNAs are marked by histone lysine methyltransfereases to create $\mathrm{H} 3 \mathrm{~K} 27 \mathrm{me} 3, \mathrm{H} 3 \mathrm{~K} 9 \mathrm{me} 2$ and H3K9me3 marks (Taverna et al. 2002). These marks are essential for the recruitment of PDD1 (Programmed DNA degradation 1) and PDD2 proteins among others which are necessary for the removal these sequences (see Figure 5) (Madireddi et al. 1996; Mochizuki et al. 2002; Taverna et al. 2002). 
The second type DNA rearrangement involves chromosome breakage (BES for breakage eliminated sequence) at sites identified by a highly conserved $15 \mathrm{bp}$ sequence (Fan and Yao 2000). Five distinct germline-derived chromosomes are fragmented into nearly 250 unique somatic chromosomes. Chromosome breakage is followed by the addition of telomeres at the end of newly formed mini-chromosomes (Yao et al. 1990). The enzymology behind chromoseome fragmentation is unknown.

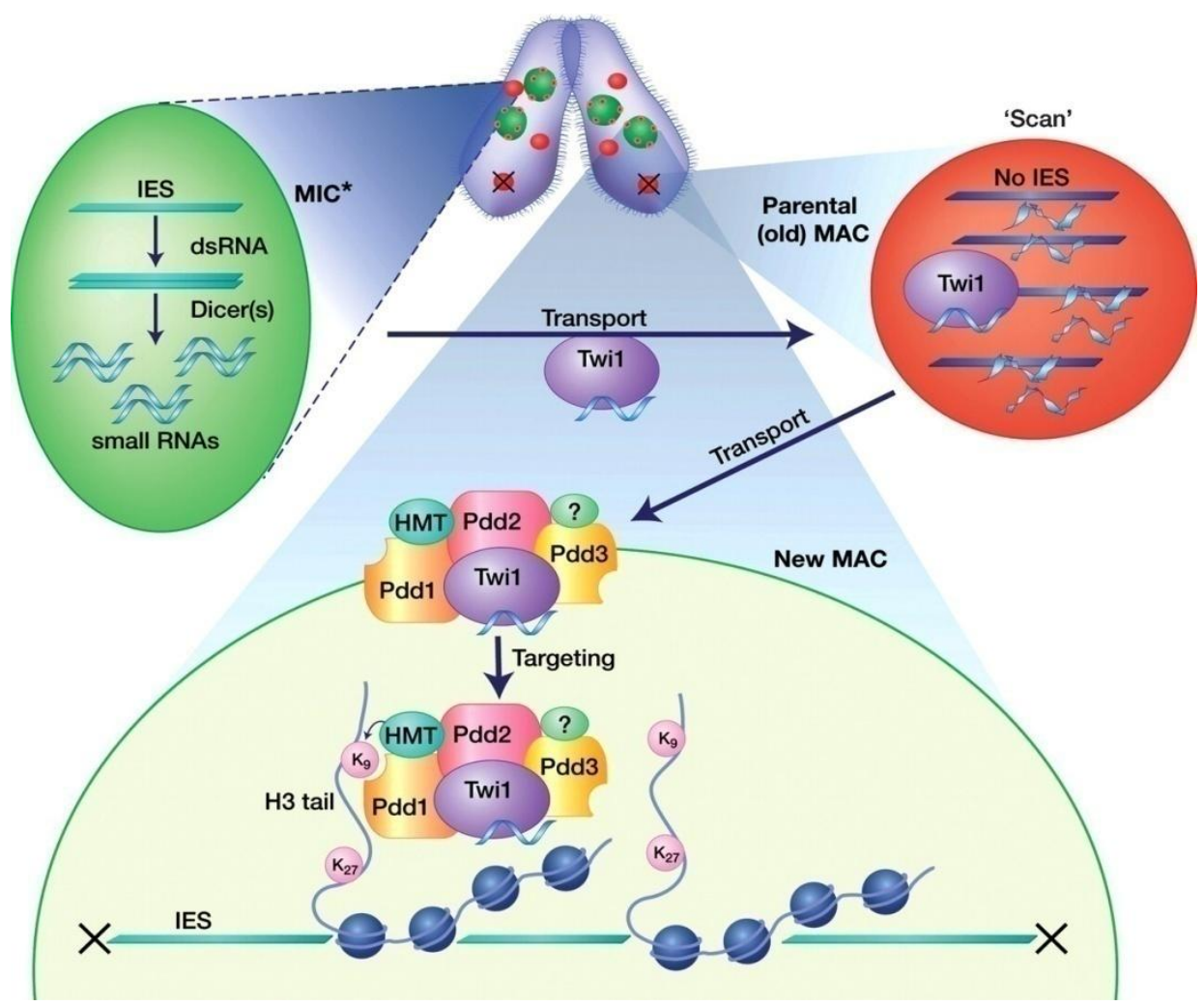

Figure 5: Scan-RNA model for IES elimination in $\boldsymbol{T}$. thermophila. The small RNAs result due to the processing dsRNA by Dicer-like ribonucleases. These are escorted by Twi1 to old MAC prior to its destruction in which IES have previously been eliminated. Only those small RNAs are retained that do not find homology in the old MAC whereas all others are degraded. These RNA are then brought back to new MAC where they target IES for elimination. See text for more details (image adopted from Bernstein and Allis 2005).

\subsection{7- T. thermophila as a model organism}

The unique biology of $T$. thermophila makes it an excellent experimental tool. Cells have a short replication time of $\sim 2$ hours and can easily be grown to large volumes in relatively 
inexpensive media. The MAC genome has been fully sequenced and annotation is available at Tetrahymena genome database (TGD: http://ciliate.org/index.php/home/welcome), making T. thermophila a suitable model organism to conduct molecular genetic studies (Eisen et al. 2006). The micro-array expression profiles for all predicted protein coding genes are also available through growth, starvation and conjugation facilitating the gene network analyses (Miao et al. 2009; Xiong et al. 2011). Furthermore, an array of molecular genetic tools has been developed to study the gene function. For example, vectors are available for gene targeting via homologous recombination that permit creation of epitope-tagged cell lines and gene knock out strains via electroporation, microinjections or biolistic transformation (reviewed in Orias et al. 2011). In addition, RNAi technology has also been developed to knockdown the genes of interest (Howard-Till and Yao 2006).

The availability of various genetic and molecular approaches has made it an ideal laboratory organism to study several cellular processes including cilia biogenesis. Its use as a model organism has led to key discoveries of several fundamental molecular and cellular processes including those of telomerase and catalytic RNA, both of which led to Nobel prizes (Kruger et al. 1982; Greider and Blackburn 1985). The nuclear dimorphism along with the polyploidy of MAC which offers a rich source of chromatin particularly makes $T$. thermophila an excellent tool to investigate processes related to DNA metabolism including chromatin assembly and gene expression regulation.

\section{5- Thesis rationale}

One of the long standing questions in molecular biology is: How does a eukaryotic cell assemble its chromatin into distinct states i.e. euchromatin - the transcriptionally active state and heterochromatin which is generally considered a silent chromatin state? Increasingly 
links are being found between defects in chromatin assembly and disease formation in humans. For example, high expression of CAF1 p60 subunit has been correlated with renal, endometrial and cervical cancer (Polo et al. 2010; reviewed in Burgess and Zhang 2013). The human HIRA gene is found in a small region on chromosome 22 that is deleted in DiGeorge syndrome which is characterized by heart defects and poor immune system (Lorain et al. 1996). Similarly, NASP miss-regulation has been correlated in a variety of cancers including those of ovary and prostrate (Ali-Fehmi et al. 2010; Ma et al. 2012). Thus a comprehensive understanding of the functions of chromatin assembly proteins may provide useful insights regarding the disease progression in humans.

Several studies have shown that cells can adopt two fundamental pathways mediated by distinct protein complexes to assemble the chromatin. For example, RD chromatin assembly is mediated by CAF1 whereas Hira functions in the RI pathway (Smith and Stillman 1991; Green et al. 2005). Despite the identification of these protein complexes, questions remain regarding the mechanistic details. For example, the exact role of epigenetic marks during chromatin assembly including those of deposition related $\mathrm{H} 4$ diacetylation is not fully clear. Furthermore, CAF1 has been implicated in the epigenetic inheritance (Ridgway and Almouzni 2000); however the exact nature of its role in this process is poorly understood. More importantly, functional link between RD and RI assembly and establishment of distinct chromatin states is still elusive.

Studying protein-protein interactions can provide useful insights into proteins' function. Recently, using affinity purification combined with mass spectrometry our laboratory has demonstrated that in T. thermophila Asf1 co-purifies with Nrp1, an Importin $\beta 3$ and two novel proteins Aip1 and Aip2 (Garg et al. 2013). These results suggested that evolutionary conserved function of Asf1 is to transport newly synthesized histones $\mathrm{H} 3$ and $\mathrm{H} 4$ through its 
physical interaction with Importin $\beta 3$. Under the experimental conditions used, $T$. thermophila Asf1 did not co-purify with any of putative CAF1 subunits or Hira which are two known interactions in other organisms. Our gene network analysis however suggested that putative Hira and $\mathrm{Cac} 2$ have similar expression profiles as of Asf1 and likely function in the same pathway (Garg et al. 2013). Clearly, more work is required to fully understand the nature of chromatin assembly pathways in this divergent model organism.

The spatial distinction of two chromatin states in T. thermophila, i.e. silent chromatin in the form of MIC and transcriptionally active chromatin in the form of MAC, offers a unique opportunity to study the molecular processes related to their assembly. Starting from histone H3/H4 supply chain, I set out to decipher the proteomic composition of $T$. thermophila RD and RI chromatin assembly complexes. By virtue of "guilt by association" which can provide insights into proteins' functions, I expected to gain insights into the mechanistic links between RD/RI assembly pathways and formation of distinct chromatin states within MIC and MAC. Using a simple model organism by analogy I hope to provide a better understanding of these processes in humans.

\section{6- Summary}

T. thermophila MAC genome encodes putative homologs of NASP, Hat1, Hat2, Cac2 subunit of CAF1 as well as HIRA as assessed by BLAST analysis (Note: T. thermophila homologs are designated with ' $\mathrm{Tt}$ ' hereafter unless unique name is defined). Specifically, my objective was to begin to characterize chromatin assembly pathways in T.thermophila via proteomic analysis of $\mathrm{Nrp} 1, \mathrm{Cac} 2^{\mathrm{Tt}}$ subunit of $\mathrm{CAF} 1^{\mathrm{Tt}}, \mathrm{HIRA}^{\mathrm{Tt}}$, Hat $1^{\mathrm{Tt}}$, and Hat $2^{\mathrm{Tt}}$. Initially, a comprehensive bioinformatic analysis was carried out in order to analyze the conserved protein sequence and structural features of the putative homologs. Subsequently, I engineered 
T. thermophila cell lines expressing C-terminal FZZ (3×FLAG-Tev-ZZ) epitope tagged NRP1, CAC2 $2^{T t}, H I R A^{T t}, H A T 1^{T t}$ and $H A T 2^{T t}$ from their native chromosomal loci. The FZZ epitope tag enabled me to perform affinity purifications for each protein of interest followed by MS analyses (AP-MS) in order to characterize their respective complexes. My results indicate that Nrp1-FZZ co-purified with $\mathrm{Asf1}^{\mathrm{Tt}}$ and two putative heat shock proteins suggesting that NASP proteomic network is conserved in this highly divergent unicellular eukaryote. My results confirmed the identity of the $T$. thermophila CAF1 complex in that $\mathrm{Cac} 2^{\mathrm{Tt}}-\mathrm{FZZ}$ co-purified with putative $\mathrm{Cac}^{\mathrm{Tt}}$ and $\mathrm{Cac} 3^{\mathrm{Tt}}$ proteins (CAF1 subunits). Additionally, I co-purified with Cac2 three putative subunits of a $T$. thermophila Casein Kinase II (CKII) complex. This suggests that CKII might be the enzyme responsible for the phosphorylation of CAF1 complex. Furthermore, MS analysis of Hat ${ }^{\mathrm{Tt}}$-FZZ showed that it co-purifies with a putative Hat $2^{\mathrm{Tt}}$ protein indicating that composition of Hat1-complex is evolutionarily conserved. Interestingly, this Hat $2^{\mathrm{Tt}}$ defined on the basis of co-purification with Hat1 is in fact the same protein that also co-purified as the $\mathrm{Cac}^{\mathrm{Tt}}$ subunit of putative $\mathrm{CAF} 1^{\mathrm{Tt}}$ complex when $\mathrm{Cac} 2^{\mathrm{Tt}}-\mathrm{FZZ}$ was used as bait. These results suggest that Hat $2 / \mathrm{Cac} 3^{\mathrm{Tt}}$ exists in the context of multiple complexes in T. thermophila and is capable of carrying out functions that are distributed between two separate genes in higher eukaryotes e.g. humans. Reciprocal verification of Hat $2 / \mathrm{Cac} 3^{\mathrm{Tt}}-\mathrm{FZZ}$ interactions with $\mathrm{CAF} 1^{\mathrm{Tt}}$ and $\mathrm{Hat} 1^{\mathrm{Tt}}$ complexes further indicated that it also interacts with the members of putative SIN $3^{\mathrm{Tt}}$ transcriptional corepressor complex. Taken together these results indicate that $T$. thermophila Hat $2 / \mathrm{Cac} 3^{\mathrm{Tt}}$ is a functionally diverse protein and is a key player in several chromatin related processes including acetylation of histone $\mathrm{H} 4$, chromatin assembly as well as transcription regulation. 


\section{Chapter 2: Materials and Methods}

\section{1- Equipment}

Room temperature centrifugations of $1.5 \mathrm{ml}$ Eppendorf tubes were carried out using an Eppendorf 5424 centrifuge whereas Sorvall Legend Micro 21R refrigerated microcentrifuge (Thermo Scientific) was used for $4^{\circ} \mathrm{C}$ centrifugations. Furthermore, Centra CL32 (IEC) and Sorvall Legend RT were employed for 15/50ml Falcon tubes centrifugations for room temperature and $4^{\circ} \mathrm{C}$ spins, respectively. In addition, large scale centrifugations (500ml) of $T$. thermophila were carried out using Avanti J-30I (Beckman Coulter). For polymerase chain reactions (PCR) GeneAmp PCR System 9700 (Applied Biosystems) and a GeneAmp PCR System 9600 (PerkinElmer) were used.

\section{2- Cell Strains}

T. thermophila cell strains of inbreeding line B, CU428 [Mpr/Mpr (VII, mp-s)] and

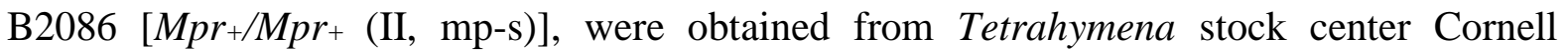
University, Ithaca N.Y. Cells were grown in axenic $1 \times \mathrm{SPP}$ media at $30^{\circ} \mathrm{C}$ as previously described.

\section{3- Bioinformatics}

\subsection{2- Sequence data retrieval}

The T. thermophila genome database (http://ciliate.org) was used to acquire the sequences of putative NRP1,HAT1 $1^{T t}, H A T 2^{T t}, H I R A^{T t}$ and $C A C 2^{T t}$. Initially, BLASTP searches were carried out against the T. thermophila sequenced MAC genome using the protein sequences of well characterized $S$. cerevisiae homologs for each gene of interest. Subsequently, each of the identified $T$. thermophila predicted gene sequence was used as a 
query to perform BLASTX searches against NCBI database of sequenced genomes (http://www.ncbi.nlm.nih.gov/) for reciprocal recoveries. All searches were performed using default parameters. See appendix 1 for accession numbers.

\subsection{3- Multiple sequence alignments, structural predictions and gene}

\section{networks}

FASTA sequences for the identified T. thermophila $\mathrm{Nrp} 1$, Hat ${ }^{\mathrm{Tt}}$, Hat $2^{\mathrm{Tt}}$, Hira ${ }^{\mathrm{Tt}}$ and $\mathrm{Cac} 2^{\mathrm{Tt}}$ were aligned against, Homo sapiens, Mus musculus, S. cerevisiae, S. pombe, Candida albicans, X. laevis, Caenorhabditis elegans and Paramecium tetraurelia homologous protein sequences. The multiple sequence alignments were built using Clustal Omega (http://www.ebi.ac.uk/Tools/msa/clustalo/) and program Jalview was used to inspect and edit the alignments (Waterhouse et al. 2009). ClustalX color coding scheme (appendix2) was used to generate the final alignments (Larkin et al. 2007). See appendix 1 for accession numbers.

Crystal structures of $S$. cerevisiae and $H$. sapiens Hat1 (PDB: 1BOB and 2POW, respectively) (Wu et al. 2012; Dutnall et al. 1998) and human RbAp46/RbAp48(Hat2) (PDB: 3CFS) (Murzina et al. 2008) were used as templates for homology modelling of $T$. thermophila Hat $1^{\mathrm{Tt}}$ and Hat ${ }^{\mathrm{Tt}}$, respectively. Alternatively, de novo structures of Nrp1, Hir $1^{\mathrm{Tt}}$ and $\mathrm{Cac} 2^{\mathrm{Tt}}$ proteins were predicted providing that the crystal structures are not yet resolved for any of these proteins. All tertiary structure predictions were carried out using I-TASSER server (http://zhanglab.ccmb.med.umich.edu/I-TASSER/) (Zhang 2008). Predicted structures were visualized using Jmol software. For functional annotation and binding site predictions, the predicted structures were submitted to CO-FACTOR server available at (http://zhanglab.ccmb.med.umich.edu) (Roy et al. 2012). Furthermore, domain analyses were also carried out using SMART (http://smart.embl-heidelberg.de/) (Letunic et al. 2012), 
'Protein homology/analogy recognition engine V 2.0' (PHYRE 2.0) (Kelley and Sternberg 2009) and PFAM database (http://pfam.xfam.org/search) (Coggill et al. 2008). In addition, theoretical iso-electric points were called by submitting the entire protein sequence to online ProtParam tool (http://web.expasy.org/protparam/).

T. thermophila gene networks were downloaded from the publically available network database available at http://tfgd.ihb.ac.cn/tool/network. These networks were constructed using context likelihood of relatedness algorithm (Z-score threshold 3.49) based on microarray expression data (Xiong et al. 2011). The downloaded networks were represented as un-directed gene networks using cytoscape version 3.02 (Shannon et al. 2003). In addition, gene expression profiles of interacting proteins indentified through mass spectrometry (see below) were compared. Hierarchal clustering was used to compare the similarities in gene expressions. The analysis was carried out using Cluster 3.0. In order to compare $T$. thermophila gene networks with those of human homologs, GeneMania web server (http://www.genemania.org/) was queried. The GeneMania utilizes a variety of sources including protein-protein interactions data, expression profiles, and co-localization data to construct the gene networks (Warde-Farley et al. 2010).

\section{4- Media, buffers, solutions}

Recipes for all media, buffers, and solutions used in this study are provided in the appendix 3.

\section{5- Growth conditions}

E. coli cells transformed with gene targeting vector pBKS-FZZ (see results section for

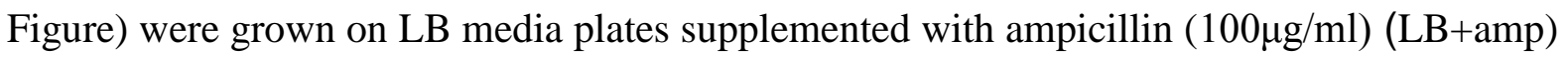


overnight at $37^{\circ} \mathrm{C}$. Subsequently plates were stored at $4^{\circ} \mathrm{C}$. In order to carry out plasmid preparation, $1.5 \mathrm{ml} \mathrm{E}$. coli cultures were overnight grown in LB+amp liquid media with shaking at $250 \mathrm{rpm}$ at $37^{\circ} \mathrm{C}$. For long term storage of cells glycerol stocks were prepared such that $0.8 \mathrm{ml}$ of overnight liquid culture was mixed with $0.8 \mathrm{ml}$ of sterile $50 \%$ glycerol in a $1.8 \mathrm{ml}$ CryoPure cryovial (Sarstedt) and stored at $-80^{\circ} \mathrm{C}$.

Vegetative T. thermophila cultures were overnight grown in sequestrin proteose peptone (SPP) media at $30^{\circ} \mathrm{C}$ with shaking at $90 \mathrm{rpm}$ using sterile flasks containing no more than $1 / 10$ of its total volume of culture. In order to carry out selection and phenotypic assortment, cells were grown in 96-well microtitre plates (Sarstedt) in SPP supplemented with the appropriate concentration of the antibiotic paromomycin. The $T$. thermophila cells were re-suspended in 10mM Tris-HCl, $\mathrm{pH} 7.4$ in order to starve them overnight. Starvation was carried out without shaking the cells.

Liquid nitrogen was used for the long term storage of $T$. thermophila. Cells were overnight grown to log phase in $100 \mathrm{ml}$ of SPP media supplemented with penicillinstreptomycin-fungizone (PSF). Subsequently, cells were washed with and re-suspended in $10 \mathrm{mM}$ Tris- $\mathrm{HCl}, \mathrm{pH} 7.4$ for starvation which continued for two days at $30^{\circ} \mathrm{C}$ without shaking in a $1 \mathrm{~L}$ Erlenmeyer flask. After two days, cells were aspirated to $250 \mu \mathrm{l}$ and $10 \%$ DMSO (Sigma) in $10 \mathrm{mM}$ Tris $\mathrm{pH} 7.4$ was immediately added (final DMSO concentration=8\%) to re-suspend the cells. CryoPure cryovials (Sarstedt) were used to store $0.5 \mathrm{ml}$ of DMSO treated cells which were then stored in liquid nitrogen.

\section{6- T. thermophila genomic DNA extraction}

Method of Gaertig et al. (1994) was used in order to extract the genomic DNA of $T$. thermophila wild type strains B2086 or CU428 (Gaertig et al. 1994). To do this, $1 \mathrm{ml}$ cells of 
T. thermophila growing in log phase were taken in $1.5 \mathrm{ml}$ Eppendorf tube and were harvested at room temperature by centrifugation at 3,000rpm for 2 minutes. The supernatant was discarded and pellet was re-suspended in $500 \mu \mathrm{l}$ of $T$. thermophila lysis solution. In order to remove the lipids and protein contents from the cell lysate phenol:chloroform (1:1) extraction was perform twice as follows: To the $600 \mu$ cell suspension $(100 \mu 1$ cell lysate $+500 \mu l$ lysis solution) $300 \mu \mathrm{l}$ phenol and $300 \mu \mathrm{l}$ chloroform was added. The solution was then mixed to homogeneity until it turned opaque. Subsequently, the solution was centrifuged at 13000rpm for $1 \mathrm{~min}$ at room temperature and top layer $(\sim 600 \mu \mathrm{l})$ was transferred to a new $1.5 \mathrm{ml}$ eppendorf tube. Then chloroform was added to it in equal amount (1:1) and solution was mixed to homogeneity. The sample was again centrifuged at $13000 \mathrm{rpm}$ for $1 \mathrm{~min}$ at room temperature and top layer $(\sim 600 \mu \mathrm{l})$ was transferred to a new $1.5 \mathrm{ml}$ eppendorf tube. Subsequently, $200 \mu \mathrm{l}$ of $5 \mathrm{M} \mathrm{NaCl}$ and $800 \mu \mathrm{l}$ of isopropanol were added to the sample in order to precipitate the DNA. The resulting sample was again centrifuged at 13,000rpm for 2 minutes at room temperature and the supernatant was discarded. The DNA pellet was then washed twice with $200 \mu \mathrm{l}$ of $70 \%$ ethanol. After the last wash, the pellet was desiccated in a vacuum desiccator for 30 minutes to remove any residual ethanol. Finally the pellet was re-

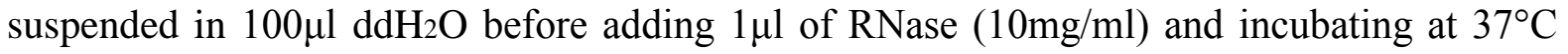
for 1 hour. The sample was stored overnight at $4^{\circ} \mathrm{C}$ and subsequently at $-20^{\circ} \mathrm{C}$.

\section{7- E. coli plasmid DNA isolation}

In order to extract the plasmid DNA from E. coli, $1.5 \mathrm{ml}$ of cultures were grown overnight. Then High-Speed Plasmid Mini Kit (GeneAid) was used to extract the plasmids in accordance with manufacturer specifications. Recovery of the extracted plasmids was 
observed using $0.8 \%$ agarose gel (made with $1 \mathrm{X}$ TBE, stained with $0.1 \% \mathrm{v} / \mathrm{v}$ of $10 \mathrm{mg} / \mathrm{ml}$ ethidium bromide for UV visualization) electrophoresis.

\section{8- Polymerase chain reaction (PCR)}

In order to perform PCR, all of reagents were added to $0.2 \mathrm{ml}$ thin-walled PCR tubes as follows to obtain a total volume of $20 \mu \mathrm{l}$ : $1 \mu \mathrm{l}$ genomic DNA, $1 \mu \mathrm{l}$ Forward Primer (30pmol/ $\mu \mathrm{l}), 1 \mu \mathrm{l}$ Reverse Primer $(30 \mathrm{pmol} / \mu \mathrm{l}), 7 \mu \mathrm{lddH} 20,10 \mu 12 \mathrm{x}$ PrimeSTAR Max DNA Polymerase (TaKaRa).

Reactions were carried out using the following conditions and recovery was assessed using gel electrophoresis:

Table 1: Table summarizes the PCR conditions used to amplify T. thermophila gene loci

\begin{tabular}{|l|l|l|}
\hline PCR step & Temperature & Time \\
\hline Denaturation & $98^{\circ} \mathrm{C}$ & 10 seconds \\
\hline Annealing & $55^{\circ} \mathrm{C}$ & 15 seconds \\
\hline Elongation & $72^{\circ} \mathrm{C}$ & 30 seconds $(\sim 5$ seconds $/ \mathrm{kb})$ \\
\hline
\end{tabular}

\section{9- DNA restriction digestions and gel extraction}

Both the plasmid and PCR products were restriction digested with appropriate enzymes (see below) using manufacturer specifications for enzymatic conditions (New England BioLabs). PCR and enzymatic cleanup of the products were carried out using an EZ-10 Spin Column PCR Products Purification Kit (Bio Basic). Furthermore, gel extractions were carried out with an EZ-10 Spin Column DNA Gel Extraction Kit (Bio Basic). Manufacturer specifications were followed to perform these tasks. 


\subsection{0- DNA ligation and transformation into competent $E$. coli}

For each ligation reaction following considerations were taken into account: linear plasmid DNA 20-100 ng, PCR insert 5:1 molar ratio over vector, 10×T4 DNA ligase buffer 2 $\mu$, T4DNA ligase enzyme $1 \mathrm{U}$ and $\mathrm{ddH} 2 \mathrm{O}$ (variable). Reaction mixture was left at room temperature for 1 hour. After this, $25 \mu$ high-efficiency competent $E$. coli cells (NEB 5-alpha, New England BioLabs) were added and transformation was carried out using the "High Efficiency Transformation Protocol" for "C2987" provided by New England BioLabs. Briefly, DNA 1-10ng was mixed with cells and left on ice for 30 minutes. Then cells were heat shocked at $42^{\circ} \mathrm{C}$ for 20 seconds after which $950 \mu 1$ SOC media was added. Cells were grown at $37^{\circ} \mathrm{C}$ for 1 hour with shaking at $250 \mathrm{rpm}$. Cells $(250-300 \mu \mathrm{l})$ spread on LB+Amp agar plates were left for overnight at $37^{\circ} \mathrm{C}$ to grow.

\subsection{1- DNA sequencing}

To verify the homology between the inserts and gene loci of interest DNA sequencing was performed by ACGT Corporation, The Centre for Applied Genomics (MaRS, The Hospital for Sick Children), and Lee Wong (Sequencing Facility, York University). The sequencing primers are listed in appendix 4.

\subsection{2- Construction of 3xFLAG-TEV-ZZ (FZZ) gene targeting vectors}

The T. thermophila gene targeting vector (provided by Dr. Kathleen Collins, University of California, Berkely, CA) contained a triple FLAG tag fused to a tobacco etch virus cleavage site fused to two repeats of the $\mathrm{Z}$ domain of the Staphylococcus aureus protein A (3xFLAG-TEV-ZZ, or FZZ). This vector is designed to target the genes for creating Cterminal tagged proteins. In order to select for the positive transformants, a neo 2 gene that 
bestows resistance to paramomycine antibiotic has also been implemented in the vector. The neo is not endogenous to $T$. thermophila so the risk of homology with the internal sites has been avoided.

Gene targeting vectors for FZZ epitope tagging were engineered as follows: $1 \mathrm{~kb}$ of DNA sequence both upstream and downstream of the predicted stop codons for each gene of interest (NRP1, HAT1 $1^{T t}, H A T 2^{T t}, H I R 1^{T t}$ and $\left.C A C 2^{T t}\right)$ was amplified using wild type $T$. thermophila genomic DNA as a template. The PCR primers were designed to contain built in restriction sites such that upstream forward and reverse primers had KpnI and Xhol sites, respectively, whereas downstream forward and reverse primers carried NotI and SacI sites, respectively (See appendix4 for primer sequences). Thus the resulting upstream and downstream PCR products for each gene of interest were digested with KpnI, Xholand NotI, SacI, respectively. Digested PCR fragments were subsequently cloned into the appropriate sites of epitope tagging vector which contained FZZ linked to a selectable drug marker, i.e. paromomycin in this case. The molecular cloning was carried out in a stepwise fashion such that upstream fragments were cloned and sequenced in the first step whereas the downstream fragments were cloned and sequenced in the second step. The resulting plasmids for each

gene were digested with KpnI and SacI restriction enzymes prior to the transformation in order to make them linear.

\subsection{3- Biolistic transformation of $T$. thermophila}

T. thermophila macronucleus transformation for the exact gene replacement was carried out using biolistic transformation method (Bruns and Cassidy-Hanley 2000) with a PDS1000/He Biolistic particle delivery system (Bio-Rad). 
Biolistic transformation was completed by growing 50ml of B2086 or CU428 wild type T. thermophila cells to $\log$ phase $\left(2 \times 10^{5}\right.$ cells $\left./ \mathrm{ml}\right)$ in SPP+PSF media with gentle shaking, pelleting the cells, washing them in $10 \mathrm{mM}$ Tris $\mathrm{pH} 7.4$ and re-suspending them in $50 \mathrm{ml}$ of 10mM Tris $\mathrm{pH} 7.4$ to allow for starvation ( 18 hours) without shaking.

Gold particles were prepared for transformation by coating $1.0 \mu \mathrm{m}$ gold beads with linearized tagging-cassette DNA $(1 \mu \mathrm{g} / \mu \mathrm{l})$. This was accomplished by adding the $3-4 \mu \mathrm{l}$ of DNA to $25 \mu \mathrm{l}$ of gold beads (per transformation), $25 \mu \mathrm{l}$ of ice cold $2.5 \mathrm{M}$ calcium chloride $(\mathrm{CaCl} 2)$, and $10 \mu \mathrm{l}$ of cold $0.1 \mathrm{M}$ spermidine (Sigma). The mixture was them vortexed at $4^{\circ} \mathrm{C}$ for 15 mins. The DNA coated gold was then pelleted and washed with $200 \mu$ of $70 \%$ ice cold ethanol followed by another wash with $200 \mu$ of $100 \%$ ice cold ethanol. Lastly, the DNA coated gold pellet was re-suspended in $20 \mu \mathrm{l}$ of $100 \%$ ice cold ethanol.

The DNA coated gold particles were then added onto flying disks which were already washed with $70 \%$ ethanol and dried. Starved T. thermophila cells were centrifuged at $3000 \mathrm{rpm}$ at room temperature and re-suspended in $1 \mathrm{ml}$ of $10 \mathrm{mM}$ Tris $\mathrm{pH} 7.4$. In order to wash and sterilize the gene gun parts $\mathrm{ddH} 2 \mathrm{O}$ and $70 \%$ ethanol were used. Starved cells were then concentrated on a pre-wet $(10 \mathrm{mM}$ Tris $\mathrm{pH} 7.4) 9 \mathrm{~cm}$ filter paper and gene gun was assembled. Briefly, a 900psi rupture disc (Bio-Rad) dipped into isopropanol and fitted into the holder which then screwed to the appropriate place. Next the flying disks contain the gold coated DNA were assembled and placed in the gun chamber using the first slot from the top. The concentrated cells were placed in the second slot from the bottom in the gene gun. Subsequently, the gene gun was operated such that the vacuum pressure was allowed to reach 25-26psi and cells were bombarded with DNA coated gold particles at $~ 900 p s i$. The cells and filter was then transferred to $50 \mathrm{ml}$ SPP media containing flasks and incubated at $30^{\circ} \mathrm{C}$ for 4 hours with gentle shaking to recover. 
After the recovery, $100 \mathrm{mg} / \mathrm{ml}$ of paramomycin was added to a final concentration of $100 \mu \mathrm{g} / \mathrm{ml}$ and cells were then transferred to a 96 -well microtitre plate, $200 \mu \mathrm{l}$ per well, and incubated at $30^{\circ} \mathrm{C}$ for 4 days. Following the incubation period, cells were observed under the microscope for robust growth which was followed by the transfer of cells to higher drug concentration. Through the gradual increase of drug concentrations, I was able to drive the phenotypic assortment such that all endogenous copies of the gene were replaced with the FZZ-tagged versions. Generally, it is achieved when cells are able to grow at $1 \mathrm{mg} / \mathrm{ml}$ of paramomycine.

\subsection{4- Western blot analysis}

In order to assess the successful expression of tagged proteins Western blot analysis was carried out. T. thermophila cell extracts were prepared using trichloroacetic acid (TCA) approach. Cells $(5 \mathrm{ml})$ were grown to $\log$ phase and $1.5 \mathrm{ml}$ were harvested in the eppendorf tubes by centrifugation at 13,000rpm at room temperature for 2 minutes. Subsequently, cells were washed with $10 \mathrm{mM}$ Tris $\mathrm{pH} 7.4$ and re-suspended in $100 \mu \mathrm{l}$ of $10 \mathrm{mM}$ Tris $\mathrm{pH}$ 7.4. Then $10 \%$ TCA (final concentration in the solution should be $1 \times$, i.e $10 \mu 1$ in this case) was added incubated in ice for $15 \mathrm{~min}$. Cells were then spun for $2 \mathrm{~min}$ at room temperature at $13,000 \mathrm{rpms}$ and supernatant was discarded. Then $100 \mu$ l of $2 \times$ SDS buffer was added to pellet was resuspended. If the color of the solution turned yellow due to acidity, $1 \mu \mathrm{l}$ of $1 \mathrm{~N} \mathrm{NaOH}$ was added. Samples were boiled for $5 \mathrm{~min}$ and kept on ice until loading or frozen at $-80^{\circ} \mathrm{C}$ for later use.

Sodium dodecyl sulfate polyacrylamide gel electrophoresis (SDS-PAGE) was used to separate the proteins based on size. The gel composition was as follows: $5 \%$ stacking gel was layered on top of $10 \%$ running gel. For each protein $10 \mu$ sample was loaded alongside $5 \mu$ of 
PiNK Plus Prestained Protein Ladder (Appendix5). The gel was electrophoresed in $1 \times$ SDSrunning buffer at a constant voltage of $100 \mathrm{~V}$. Subsequently, the proteins were transferred on PVDF membrane (Bio-Rad) activated in 100\% methanol using 1x Western transfer buffer at $10 \mathrm{~V}$ overnight or $75 \mathrm{~V}$ for 2 hours. Following the transfer of proteins membrane was blocked in $5 \%$ Blotto for 1 hour at room temperature which was followed by three 5 minutes washed in $1 \mathrm{x}$ PBS to remove any unbound milk proteins.

The probing of FLAG tagged proteins was achieved by incubating the Western blots with monoclonal mouse $\alpha$-FLAG (Sigma-Aldrich) primary antibody diluted 1:500 in 5\% milk solution. For loading controls, monoclonal mouse $\alpha$-actin (GenScript) primary antibody diluted 1:1000 in 5\% milk was used. Both incubations were carried out for 1 hour at room temperature. After the incubation, blots were washed three times ( 5 minute each) using $1 \mathrm{x}$ PBS. Subsequently, blots were incubated with horseradish peroxidase-conjugated polyclonal goat $\alpha$-mouse (Cedarlane) secondary antibody diluted 1:3000 in 1\% milk solution for one hour at room temperature. Following the three washes as described above, blots were visualized using Denville Scientific's HyGLO Chemiluminescent HRP AntiBody detection Kit (E2500) according to manufacturer specifications.

\subsection{5- Tandem affinity purification (TAP)}

TAP was carried out to isolate the FZZ tagged proteins along with their interacting partners. It should be noted that in the initial parts of the project, TAP which is a two step purification procedure was used (below), however subsequently I switched to one step affinity purification method. In was done in order to increase the probability of capturing any transient interactions that might be lost during a two step procedure. Details of any modifications made in the original TAP procedure are also provided below. 
T. thermophila cells harvested at $3 \times 10^{5}$ cells $/ \mathrm{ml}$ and grown in $\sim 500 \mathrm{ml} 1 \times \mathrm{SPP}$ were used as the starting material for the TAP. In order to carry out the TAP procedure, $25 \mathrm{ml}$ of $2 \times$ Lysis buffer as well as $50 \mathrm{ml}$ of $1 \times$ Lysis buffer was prepared. To each of these buffers, $500 \mu 1$ of protease inhibitor (sigma) $+200 \mu 1100 \mathrm{mM}$ PMSF made in isopropanol was added just before lysing the thawed cells. Frozen cell pellets were thawed on ice and were resuspended in an equal volume of ice-cold 2x lysis buffer+protease inhibitors. Subsequently, the total volume was adjusted to $15 \mathrm{ml}$ with $1 \mathrm{x}$ lysis buffer+protease inhibitors. To this, $300 \mu \mathrm{l}$ of $10 \% \mathrm{NP}-40($ final $[\mathrm{C}]=0.2 \% \mathrm{v} / \mathrm{v}$ ) and $5 \mu \mathrm{l}$ of benzonase nuclease (Sigma) was added. To allow benzonase digest all the released genomic DNA tubes were rotated end-to-end for 1 hour at $4^{\circ} \mathrm{C}$. After one hour, whole cell extracts (WCE) were clarified by dividing them into $1.5 \mathrm{ml}$ eppendorf tubes and centrifuging at maximum speed for 30 minutes at $4^{\circ} \mathrm{C}$. Then the clarified supernatants were pooled together and $100 \mu$ of WCE for each sample were separated as the input materials. The IgG-Sepharose (chromatography resin for the first step of affinity purification) was prepared (see below) and added to the pooled supernatants.

The IgG-Sepharose is supplied in an ethanol solution that acts as a preservative. Therefore it needs to be washed and equilibrated in the lysis buffer prior to use. For each affinity purification sample $250 \mu 1(200 \mu 1$ will also work) of packed beads volume (PBV) of the IgG-sepharose were used. Beads were washed three times with 1x lysis buffer and subsequently were re-suspended in an equal volume to create 1:1 beads: buffer slurry. Then the slurry for each sample was added to respective clarified WECs and samples were set on end-to end rotations for 4 hours.

After 4 hours of rotation, samples were centrifuged at $4,000 \mathrm{rpm}$ for 5 minutes at $4{ }^{\circ} \mathrm{C}$. Beads were washed once with $20 \mathrm{ml}$ of $300 \mathrm{mM} \mathrm{NaCl}$ wash buffer (IPP300) and twice with $15 \mathrm{ml}$ of $1 \times \mathrm{Tev}$ buffer. After the final wash, the beads were transferred to separate $1.5 \mathrm{ml}$ 
eppendorf tubes along with $750 \mu 1$ of $1 \mathrm{x}$ TEV buffer. Beads were again washed and resuspended in an equal volume of $1 \mathrm{x}$ TEV buffer. Then $8 \mu 1$ of TEV Protease enzyme $(2 \mathrm{mg} / \mathrm{ml})$ (kind gift from Dr. J. Greenblatt lab, University of Toronto) was added to each tube and samples were set on end to end rotation for overnight at $4{ }^{\circ} \mathrm{C}$.

Next day, the samples were centrifuged at 2,000rpm for 2 minutes at $4^{\circ} \mathrm{C}$, and the supernatant (TEV elute) was transferred to individual ice-cold $1.5 \mathrm{ml}$ eppendorf tubes. Then $600 \mu 1$ of IPP100 buffer was added to the remaining beads and following a quick spin elutes from each sample were transferred to the previous supernatants in the ice-cold $1.5 \mathrm{ml}$ eppendorf tubes.

In the second round of purification, M2-agarose beads were used. For each purification sample, $30 \mu 1$ of M2-agarose was used. Because these beads come as a 1:1 mixture with ethanol, $60 \mu 1$ of slurry was required for each sample. M2-agarose beads were washed three times with IPP100 buffer by rotating them for 3 minutes at $4{ }^{\circ} \mathrm{C}$ and centrifuging at 3,000rpm for 2 minutes at $4{ }^{\circ} \mathrm{C}$. After the last wash, beads were re-suspended in an equal volume of IPP100 buffer. The slurry was then aliquoted into pre-chilled eppendorf tubes depending upon number of samples and TEV elute from each sample was then added to the respective tube. The tubes were rotated end-to-end for three hours at $4{ }^{\circ} \mathrm{C}$.

After three hours, beads were washed once with IPP100 buffer followed by two washes with IPP100 made without NP40. The pellet was then washed once with $750 \mu 1$ of $2 \mathrm{mM}$ $\mathrm{CaCl} 2 / 20 \mathrm{mM}$ Tris and centrifuged at 5000rpm for 1 minute at room temperature. After removing the supernatant, $500 \mu \mathrm{l}$ of $0.5 \mathrm{M} \mathrm{NH} 4 \mathrm{OH}$ was added and tubes were rotated for 20minutes at room temperature. This was followed by centrifugation at 5000rpm for 2 
minutes at room temperature. The supernatant was transferred to separate pre-chilled eppendorf tubes and stored at $-80^{\circ} \mathrm{C}$ for $\mathrm{MS}$ and Western blot analyses.

In the modified one step affinity purification procedure the IgG-Sepharose binding and TEV cleavage steps were omitted. The clarified WCEs were directly transferred to M2agarose beads. In this case $50 \mu 1$ of to M2-agarose beads washed with 1x lysis buffer were used for each purification sample. The rest of the procedure was similar to the one mentioned above.

\subsection{6-Mass spectrometry}

The MS analysis was carried out by Dr. J.P. Lambert at a collaborating laboratory of Dr. Ann-Claude Gingras in the Lunenfield-Tanenbaum Research Institute at Mount Sinai Hospital. Speed-vac without heating was used to dry TAP purified proteins eluted in $0.5 \mathrm{M}$ $\mathrm{NH}_{4} \mathrm{OH}$. Trypsin digestion was carried out in a solution to cleave the peptides. Capillary column $(75 \mu \mathrm{m}$ id) packed in-house with 10cm Reprosil-Pur 120 C18-AQ, 3uM (Dr-Maisch $\mathrm{GmbH}$; Germany), pre-equilibrated with $2 \%$ acetonitrile (ACN) and $0.1 \%$ formic acid was used to manually bomb load the trypsin digested peptides. This reversed-phase highperformance liquid chromatography column was placed in-line with a linear trap quadrupole (LTQ) mass spectrometer via an electrospray ionization delivery system. Tandem mass spectrometry (MS/MS) was used to analyse the ionized species. The parameters for data dependent acquisition on the mass spectrometer were: 1 centroid MS (mass range 400-2000) followed by MS/MS on the 5 most abundant ions. The statistical evaluation program Mascot version 2.3 was used against the $T$. thermophila RefSeq protein database (NCBI) to analyse the resulting data files. The fragment mass tolerance was $0.6 \mathrm{Da}$ (monoisotopic mass) and the mass window for the precursor was +/- 3Da average mass. The ion score cut off was 35 and a 
protein hit must have two "bold red peptides" to be considered, where red indicated that peptide was the top scoring match, and bold indicated that the protein was the highest scoring match in which the peptide was found.

In order to provide a statistical significance to individual protein-protein interactions, data was subjected to SAINT analysis using SAINT (Choi et al. 2012). This method takes quantitative spectral counts into consideration for assigning a value to individual proteinprotein interactions. Depending upon the quantitative data, SAINT algorithm assumes that a prey protein captured with bait is either its true interactor or a non-specific binder. In order to qualify as a true interactor, the prey must be present in significantly higher abundance than the abundance in the negative controls. Thus by comparing data from negative control and experimental AP-MS, a probability value to an interaction is assigned and a rigorous discrimination between true and false interactions is achieved when data from biological replicas is taken into consideration. An average probability (AvgP) is reported using individual experimental replicas (Choi et al. 2012) and a value greater than 0.8 is considered statistically significant in this study.

\subsection{7- Indirect Immunofluorescence}

For indirect immunofluorescence, vegetatively growing cells were harvested, fixed in $4 \%$ paraformaldehyde and membrane-permeabilized with cold acetone for 20 min. Incubation with primary mouse anti-FLAG antibody (sigma) or anti-GFP antibody was at a 1:500 dilution at $4^{\circ} \mathrm{C}$ overnight in $1 \times$ PBST. Secondary antibody was fluorescein isothiocyanateconjugated goat anti-mouse (Pierce) for $1 \mathrm{~h}$ at room temperature. For nuclear counterstaining 4,6-diamidino-2-phenylindole dihydrochloride was used. 


\section{Chapter 3: Results}

In order to learn about chromatin assembly in T. thermophila, I initiated a proteomic analysis of the conserved, major histone $\mathrm{H} 3-\mathrm{H} 4$ chaperones including $\mathrm{Nrp} 1, \mathrm{Cac} 2^{\mathrm{Tt}}$ and $\mathrm{Hira}^{\mathrm{Tt}}$ as well as putative histone modifying Hat1/Hat ${ }^{\mathrm{Tt}}$ complex. I first engineered five DNA constructs/gene targeting vectors to target the native chromosomal loci of each gene of interest. The tagging vectors were constructed by amplifying two separate $\sim 1 \mathrm{~kb}$ fragments up and downstream of the predicted stop codons for each gene of interest (See Figure 6-A). The amplified sequences were cloned into the appropriate sites within the tagging vectors which contained an FZZ (3xFLAG-TEV-ZZ) epitope tag (See Figure 6-B).

Subsequently this DNA construct was used to transform growing $T$. thermophila strains by biolistic transformation method (Gaertig and Gorovsky 1992). By virtue of homologous recombination, the FZZ tag is inserted to C-terminus of the native chromosomal locus of interest (See Figure 6-C). The polyploid MAC divides through amitosis and lacks a mechanism for the equal segregation of alleles. Therefore, the process of phenotypic assortment generates eventual homozygocity in the polyploid MAC for the chromosome carrying epitope tagged locus. This process can be accelerated by step wise increase in the

drug concentration (See Figure 4), as long as the epitope tag does not itself has a deleterious phenotype when added to the $\mathrm{C}$-terminus of the gene in question. 
A

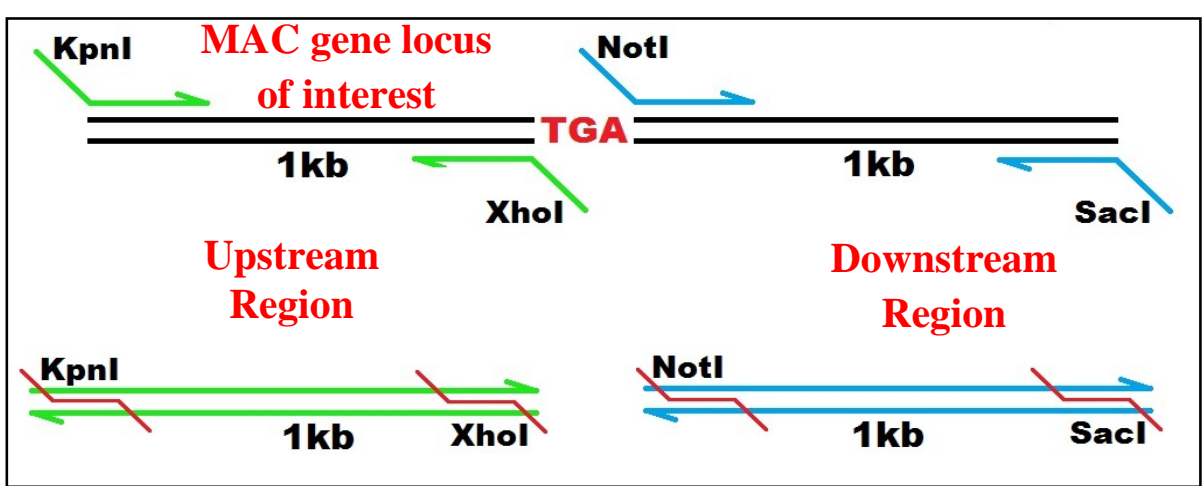

B

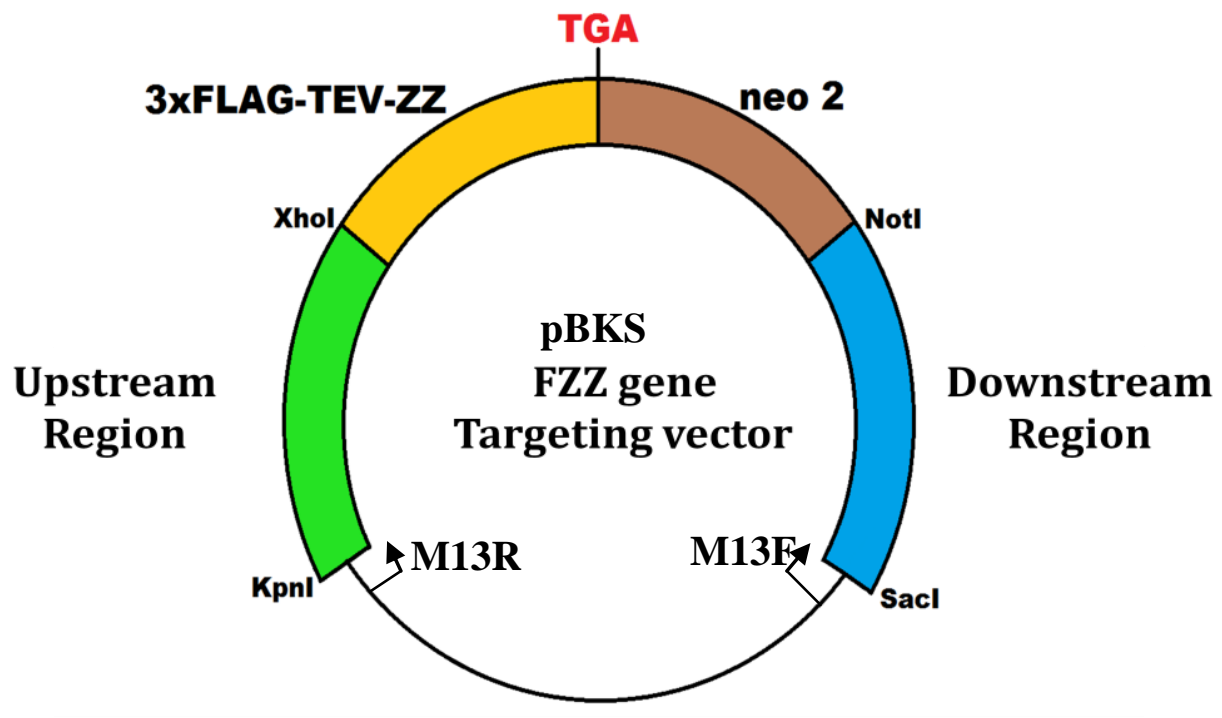

C
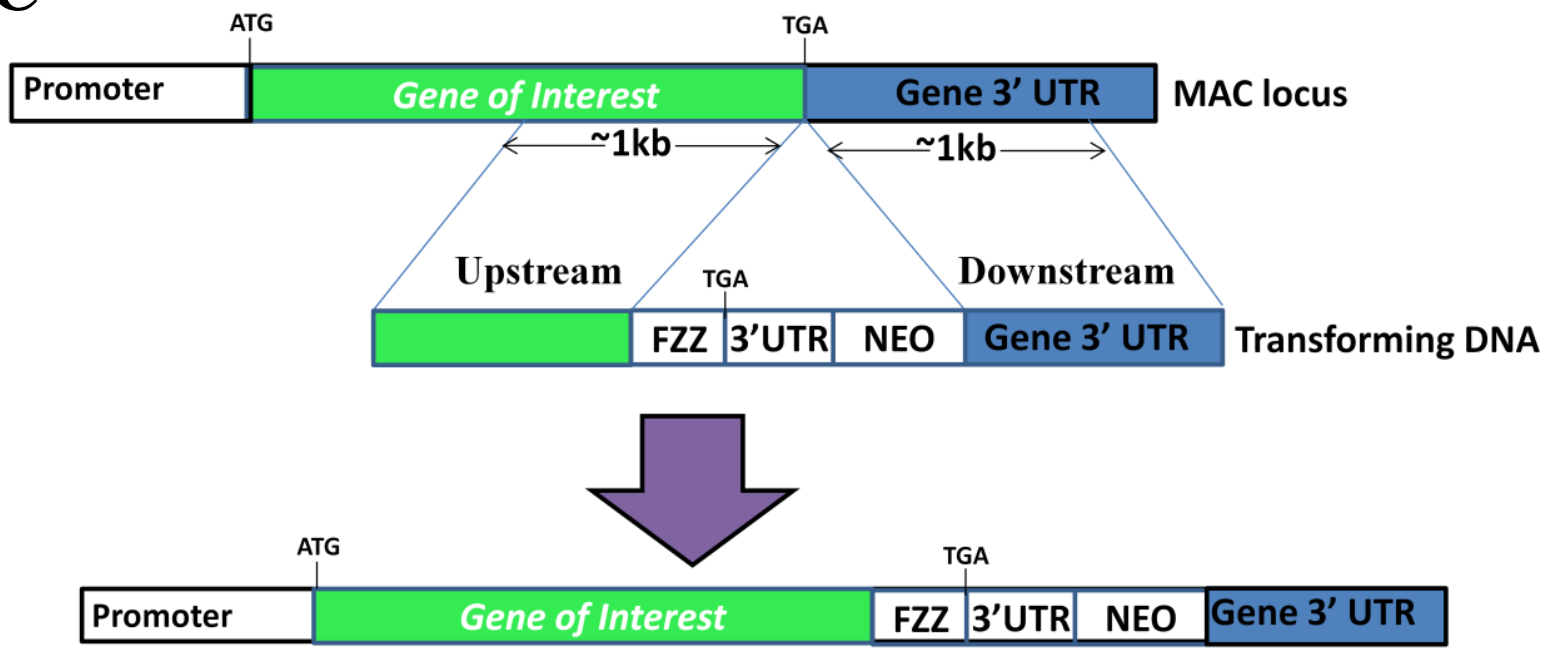

Figure 6: Schematic representation of gene tagging vector construction. A- Position of PCR primers along with built-in restriction sites are shown. B- Simplistic map of the gene tagging vector. Different regions are shown in colors. TAG represents stop codon and position of sequencing primers M13R/F is also shown. C- Insertion of FZZ tag at the Cterminal to the MAC locus via homologous recombination is shown. 


\section{1- Characterization of Nrp1}

\subsection{2-Nrp1 has conserved motif architecture}

NASP family proteins have previously been shown to be conserved from yeast to mammals (Dunleavy et al. 2007). The unique feature of NASP is that it contains four TPR motifs (TPR1-4) where the second TPR is interrupted by acidic patches (SHNi-TPR) (Dunleavy et al. 2007). Recently, our laboratory showed that in T. thermophila Asf ${ }^{\mathrm{Tt}}$ physically interacts with Nrp1 suggesting that NASP-Asf1 interaction is evolutionary conserved (Garg et al. 2013). To assess the presence of TPRs in this newly identified NASP family member I aligned its predicted protein sequence against well characterized $H$. sapiens, S. cerevisiae, S. pombe and X. laevis NASP homologs. A putative NASP homolog from a related ciliate $P$. tetraurelia is also included in this multiple sequence alignment (MSA) analysis.

The resulting MSA revealed that the Nrp1 TPR motif architecture is well conserved where the interrupted TPR2 is flanked by TPR 1 and TPR3/4 (see Figure 7). This arrangement is in accordance with the motif architecture of SHNi-TPR family (Dunleavy et al. 2007). Although the overall sequence identity across various lineages is rather low, TPRs 1-4 sequences appear well conserved (>40\% similarity with human NASP, see Figure 8 AD). In accordance with Dunleavy et al. (2007), the second residue of each TPR and $9^{\text {th }}$ residue of TPR3 in Nrp1 is either acidic or amidated. The MSA analysis also revealed certain residues that have remained highly conserved across various lineages. For example, in each TPR motif positions 7 and 8 are well conserved and thus possibly represent functionally important sites. In addition, positions with variable amino acids have retained some of their biochemical properties (e.g. Hydrophobic, aromatic etc) (see Figure 8 A-D). 


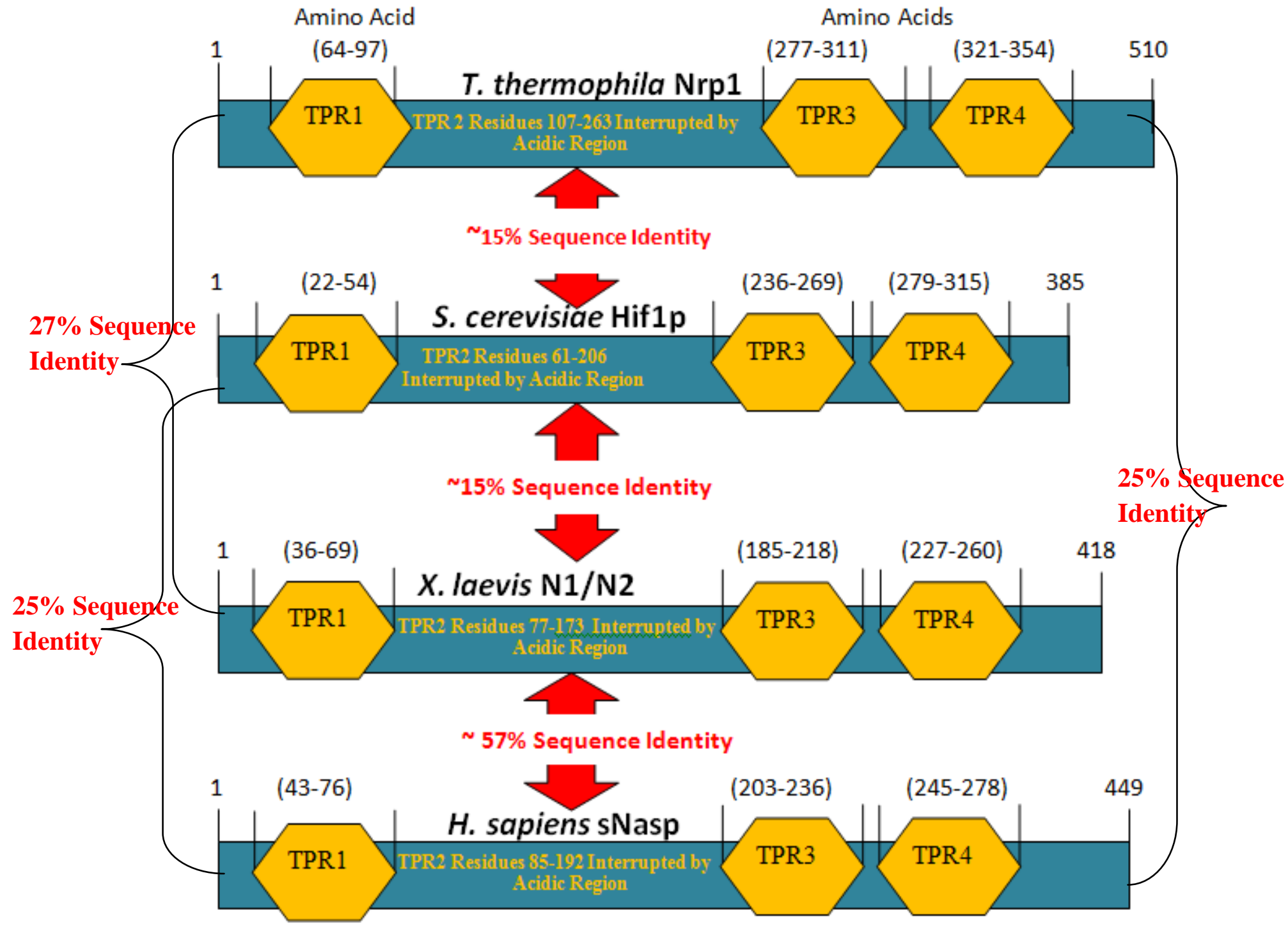

Figure 7: NASP TPR motif architect across various eukaryotic lineages. Sequence identities (\%) as well as the boundaries of TPRs 1-4 are shown. The figure is generated based on MSA analysis and amino acid residues comprising the TPRs are indicated. 
H. sapiens/1-34

X. laevis/1-34

S. cerevisiae/1-34

S. pombe/1-34

T. thermophila/1-34

P. tetraurelia/1-34
A KKL LGLGQKHLVMGD I PAA VNAFQEAA S LLGKK

A KRLMGAGQK HL VMKD VR S A VNLFQEA S S LLAKQ

MERQVQ I AKD LLAQKK F LEAAKRCQQT L D S L PKD

I EQL VTQGNMAYAQKN YEEA VDKYGQA L MQSES I

LESRL TEAVECYKNKN FDQAEN I FSDLM I YAVKF

AKEL F NEGKHNEA EVMMQGVMQQALAYY KDELAV

B

H. sapiens/1-34

X. laevis/1-34

S. cerevisiae/1-34

S. pombe/1-34

T. thermophila/1-34

P. tetraurelia/1-34
GEAF F F YGK S LLE LAR---LELAW DMLDLAK I I F KRQ
AEAF Y S YGM S LLE LAR---LQLAW EMLDLCK I I F KRQ
PELF T I FAQ A VYNMEV---YENAL D L LAQALML LGRP
RNVLW L YGK S LFQ I A I ---DFNV A W E VLDL T R VMQSK
SKYF F F YGD MLLS KLE---LQLAW E NLELSR K I YNQE
AEYYYMYG T I I VL KL S---FHI AW E NLEVAR V I LEKE

C

H. sapiens/1-34

X. laevis/1-34

S. cerevisiae/1-34

S. pombe/1-34

T. thermophila/1-34

P. tetraurelia/1-34
AQAHLK L GEVS VE SENYVQAVEEFQ S CLN LQEQY

AQAHQK L GEVC I E SENYSQAVEDFL ACLN I QKEH

GDVY I LMGD I ER E AEMF SRA I HHYL KALG Y YKTL

AD I YDL L GEL S L E I E N F SQASQDLK T ALEWKEKV

VEVTLR I ADLEQWRDNFSDALKEYTMALQL SKE I

AKVY I K L AELDQWRDK FDDAQENLQK SLQ L RLQC

D

H. sapiens/1-34 AETHYQLGLAYGYNSQYDEAVAQFS KS I EV I ENR

X. laevis/1-34

S. cerevisiae/1-34

S. pombe/1-34

T. thermophila/1-34

P. tetraurelia/1-34

\section{AETHYHLGLAYQY S S KHEEA I SHFTQS I GV I EKR}

I QAEFLVCDALRWVDQVKDKLKRFKHAKALLEKH

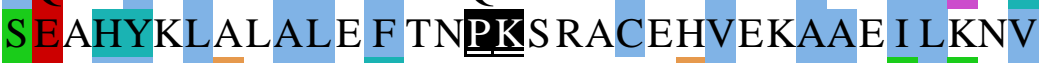
I F FQLGNAVL Y ENKEYCEEEGLKNF I DS AQ I L

AETYFFLGNVTLYNYKEGKEEEALGFYLKALQ I L

Figure 8: Multiple sequence alignments of TPRs 1-4 shown in A-D respectively. The Second residue in each TPR and the $9^{\text {th }}$ residue in TPR 3 are highlighted in red to indicate that these residues or either acidic or amidated. The acidic interruption in TPR2 is not shown for clarity however it has been indicated as gaps. In addition, TPR4 in S. pombe contains a small insertion which is not shown but its position has been indicated by highlighting the residues in white against black background. The color coding scheme is based on clustalX (see appendix 2). 
Crystal structural analysis has revealed that TPRs are 34 amino acid long amphipathic helices that form a helix-turn-helix arrangement (D'Andrea and Regan 2003). The TPRs are ubiquitous motifs found in a large number of proteins and are thought to provide structural scaffold to mediate protein-protein interactions (D'Andrea and Regan 2003). In order to visualize the structural features I submitted the primary amino acid sequence of Nrp1 to the I-TASSER server with default parameters which predicted a de novo three dimensional structure (See figure 9 below). The predicted structure consists of large amount of alphahelices and the TPR 1-4 consist of two anti-parallel alpha helices generating an overall groove surface. Notably, the TPR1 and TPRs 3-4 adopt a classical TPR fold of helix-turnhelix arrangement (see PDB: 1FCH) whereas TPR2 has an extended topology due to the acidic insertions. The acidic region is mostly disordered and surrounds the outer surface of TPR2 further facilitating the formation of an overall grooved structure. T. thermophila Nrp1 has 110 acidic residues (glutamic and aspartic amino acids) giving it an overall net negative charge with a theoretical iso-electric point (PI) of 4.58. This correlates well with the human sNASP which harbours 99 acidic residues with a PI of 4.35. Interestingly, 40 out of 110 acidic residues are found in the TPR2 insertion of $T$. thermophila Nrp1 making it highly negatively charged (PI: 4.12). The overall structure along with negatively charged extended loops of TPR2 acidic region suggest that positively charged histones $\mathrm{H} 3 / \mathrm{H} 4$ may interact by falling into the groove due to favourable electrostatic interactions. Nrp1 also contains a coiled-coil domain (CCD) as predicted by PHYRE 2 server and shown in the Figure 9. The functionally diverse CCDs are often found to mediate protein oligomerization and to provide structural stability. Previous reports have suggested that CCD in human NASP might be responsible for its dimerization (Finn et al. 2012). 


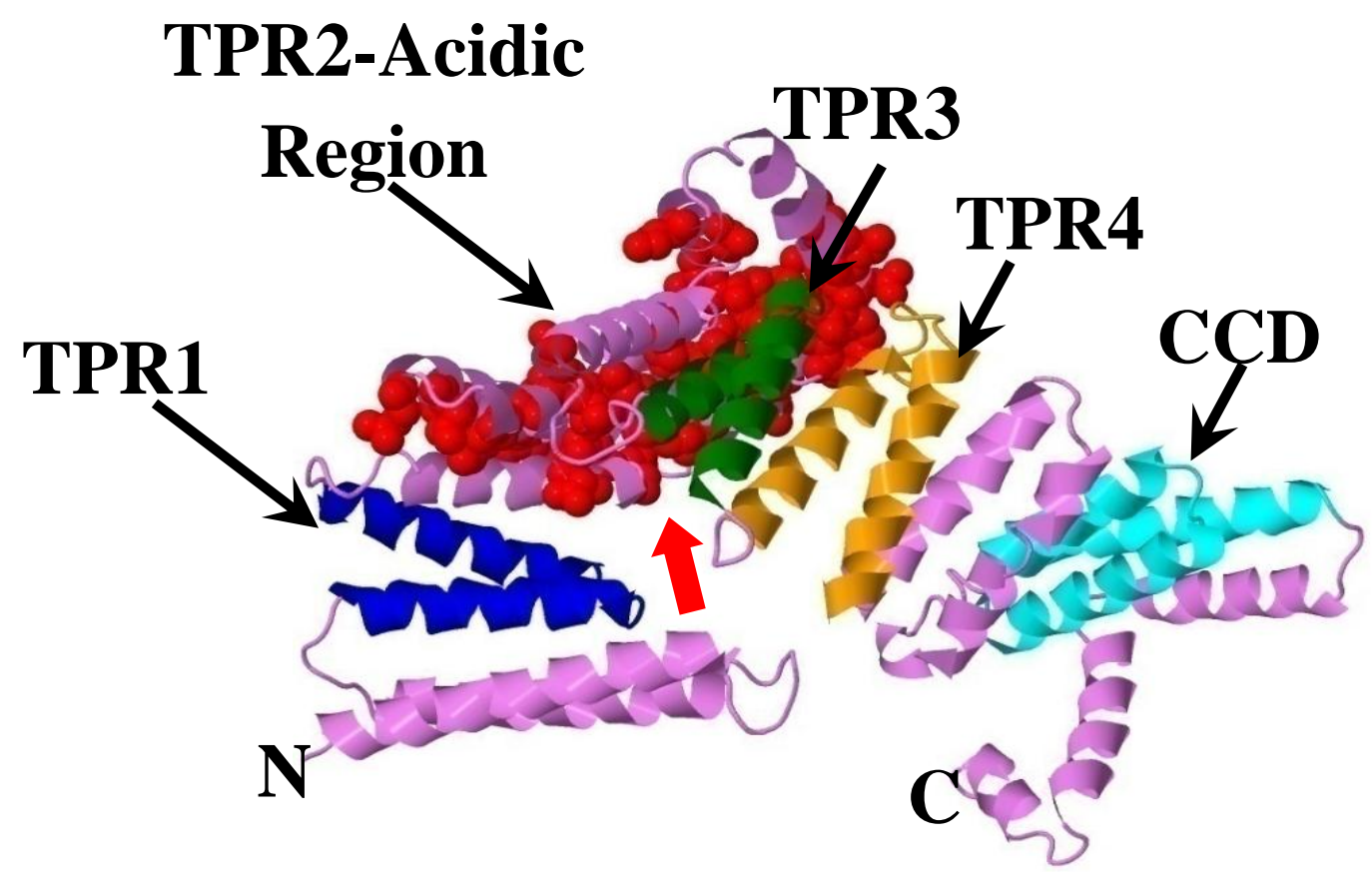

Figure 9: Predicted structure of Nrp1 is shown in ribbon representation. Predicted TPRs 1,3 and 4 are highlighted in blue, green and gold colors, respectively, whereas acidic residues interrupting TPR 2 are indicated in red CPK models. The position of a predicted CCD found towards the C-terminus is also highlighted. Furthermore, the position of another CCD (150-189 amino acid) predicted by SMART is not shown. The dark red arrow indicates that possible groove in the Nrp1 structure for $\mathrm{H} 3 / \mathrm{H} 4$ interaction. 


\subsection{3- Nrp1 Protein-Protein interactions in growing cells}

In order to identify the protein interaction network of Nrp1, I generated stable $T$. thermophila cell lines expressing from its MAC locus Nrp1 with a C-terminal FZZ epitope tag. The FZZ epitope tag carries a TEV cleavage site that is flanked by 2 protein A moieties (Z) and one 3xFLAG (F). This double epitope tag arrangement allows for tandem affinity purification of the fusion proteins permitting analysis of co-purifying partners by mass spectrometry. Successful epitope tagging was assessed by confirming expression of the FZZ-tagged protein on a western blot of whole cell extracts made from both Nrp1-FZZ and wild type strains (Figure 10).

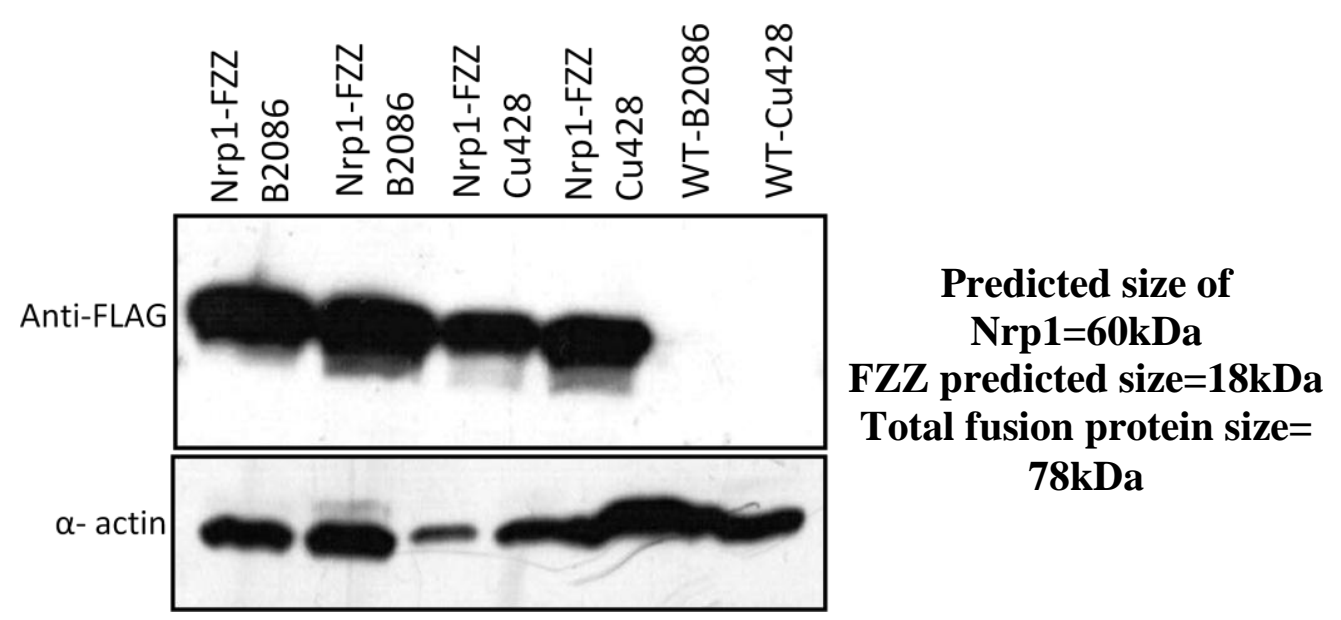

Figure 10: Western blot of Nrp1-FZZ indicating the successful expression of tagged protein. Top panel was probed with anti-FLAG antibody (M2) which showed a strong signal in the lanes corresponding to Nrp-FZZ in T.thermophila opposite mating types. The bands appeared within the reasonable range of the fusion protein predicted size $(\sim 78 \mathrm{kDa})$. The lower panel was probed with anti-actin antibody as a loading control.

Subsequently tandem affinity purification was performed on whole cell extracts generated from Nrp1-FZZ and wild type strains. The successful recovery of tandem affinity purified Nrp1 was assessed by Western blotting (see Figure 11). As apparent from the figure 11, the size of the Nrp1 in the input lane is greater than the TAP lane. This difference is size represents the successful removal of protein A part of the FZZ tag, 8kD. 


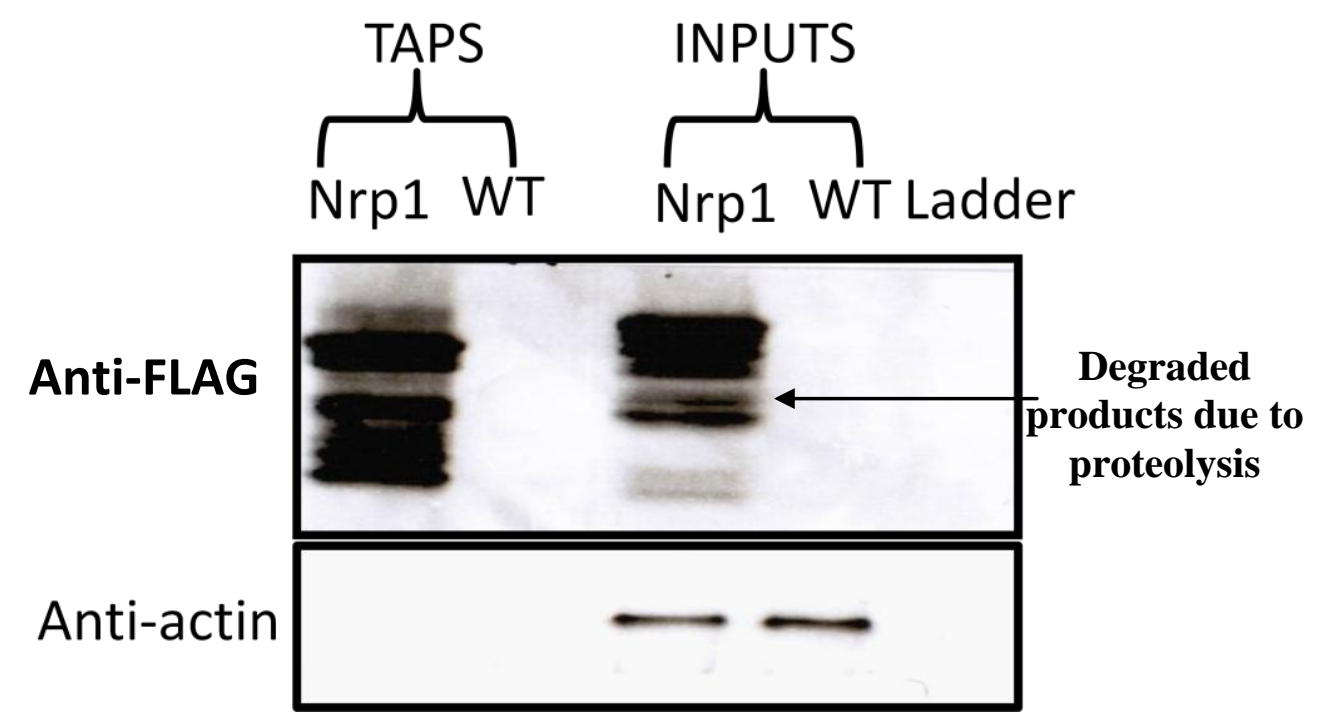

Figure 11: Successful recovery of purified Nrp1 protein detected by Western blot. The top panel was probed with anti-FLAG antibody to detect the signals in the Nrp1 input and TAP lanes. In contrast no signal was detected in the wild type lanes. Note, the size of Nrp1 in the TAP lane is smaller than the input lane indicating the removal of protein A part of the tag. The bottom panel was probed with anti-actin antibody as a loading control. Signals were detected only in the input lanes and not in the TAPs lanes.

Importantly, AP-MS was repeated at least three times to provide experimental replicas for the downstream statistical analysis (SAINT). SAINT analysis of the AP-MS data from vegetative $T$. thermophila cells revealed three Nrp1 interacting partners with AvgP > 0.9 (see methods for details). Table 2 summarizes the mass spectrometry data from three sets of Nrp1 affinity purifications. The "Gene ID" refers to T. thermophila accession numbers whereas spectral count represents the number of total spectra identified in each round of mass spectrometry analysis. In accordance with our previously reported Asf1-Nrp1 interaction (Garg et al. 2013), Asf1 (TTHERM_00442300) was recovered with an AvgP=0.99 in Nrp1FZZ purifications. This result reciprocally verifies Nrp1-Asf1 interaction identified in Garg et al. (2013). SAINT analysis also revealed Nrp1 interaction with two putative heat shock proteins (dnaK) TTHERM_00688300 and TTHERM_00105110 with an AvgP=1 and 0.94, respectively. Both of these proteins share homology to HSP70 by BLAST searches (data not shown). These observations suggest that similar to human NASP, Nrp1 physical interaction 
with heat shock proteins (HSP90/70) is conserved and might have a role in newly synthesized $\mathrm{H} 3 / \mathrm{H} 4$ transport mechanism.

It should be noted that a large number of spectral counts for $\mathrm{H} 3$ and $\mathrm{H} 4$ were also detected in Nrp1-FZZ purifications. Histones are highly abundant proteins and often are also found as background contaminants. In fact histones are well known "frequent flyers" in MS data along with ribosomal proteins (Choi et al. 2012). Due to this reason they did not pass the SAINT threshold and were filtered out from the final data set.

Table 2: AP-MS raw data filtered using SAINT analysis. The AvgP represents the overall probability of individual experimental replicates.

\begin{tabular}{|l|l|l|l|l|l|}
\hline Bait & Prey & Gene ID & \multicolumn{2}{l|}{ Spectral count } & AvgP \\
\hline & & & Sample & Control & \\
\hline Nrp1-Veg & Nrp1 & TTHERM_01014770 & $247|657| 180$ & n.d & \\
\hline Nrp1-Veg & dnaK & TTHERM_00688300 & $8|12| 8$ & n.d ${ }^{*}$ & - \\
\hline Nrp1-Veg & dnaK & TTHERM_00105110 & $15|32| 16$ & $0|4| 0|0| 3|0| 7|3| 0|0| 0|3| 0$ & 0.94 \\
\hline Nrp1-Veg & Asf1 & TTHERM_00442300 & $4|4| 4$ & n.d & \\
& & & & & 0.99 \\
\hline
\end{tabular}

* none detected in 9 vegetative cell control

\subsection{4-Nrp1 localization in MIC is cell cycle dependent}

Studying sub-cellular localization patterns of a protein has the potential to yield useful insights into protein's proteins. In order to assess the functional roles of Nrp1 in growing cells I performed an indirect immunofluorescence (IF) analysis by generating a stable $T$. thermophila cell line expressing GFP tag from the native chromosomal locus of Nrp1 in the MAC. IF analysis indicates that Nrp1 primarily localizes to the MAC during 
vegetative growth (see Figure 12-Top panel). This observation suggests that Nrp1 might be functioning as a storage buffer for soluble histones $\mathrm{H} 3$ and $\mathrm{H} 4$ consistent with the previous findings in human cells (Cook et al. 2011).

During vegetative growth MAC and MIC divide by different mechanisms, amitosis and mitosis respectively, and at different stages of the cell cycle. Typically, during interphase MIC sits in a pocket-like recess in the MAC surface and this position signifies interface MAC and MIC (reviewed in Wei et al. 1998). At the initiation of mitosis, the MIC starts to move away from MAC. Once MIC finishes dividing, macronucleus initiates its division and MIC enters $S$ phase of cell cycle due to the lack of a distinct G1 phase. This $\mathrm{S}$ phase continues for a short period of time after the division of MAC and ends with cytokinesis (reviewed in Wei et al. 1998). Interestingly, during IF analysis, I observed Nrp1 localization to the MIC when it starts moving away from MAC (see Figure 12). This staining persists till the end of cytokinesis suggesting that Nrp1 is present in MIC during S-phase. These results suggest that Nrp1 localization to distinct nuclei is cell cycle dependent possibly representing its role in the influx of histones to the MIC during S-phase. Taken together data presented here suggests that Nrp1 is a member of NASP family proteins and functions as a $\mathrm{H} 3 / \mathrm{H} 4$ chaperone through its conserved physical interactions with Asf1 as well as heat shock proteins. 
A

\section{Nrp1-GFP}

\section{DAPI}
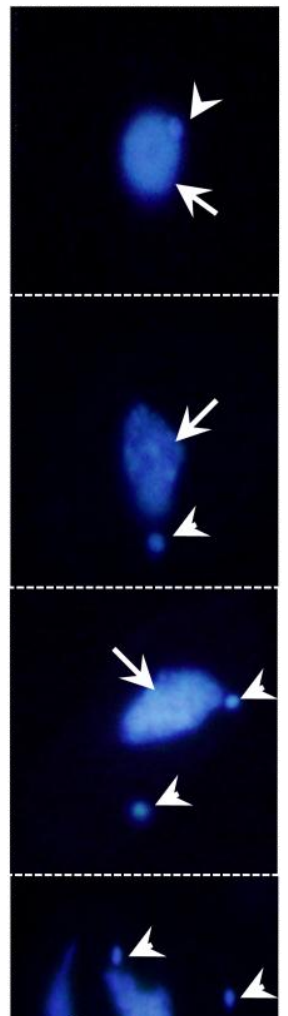

$k \pi$
FITC

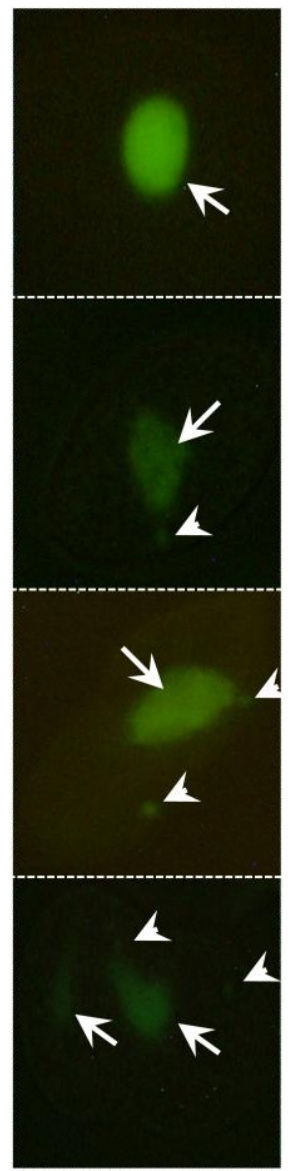

B

\section{Un-tagged}

DAPI FITC
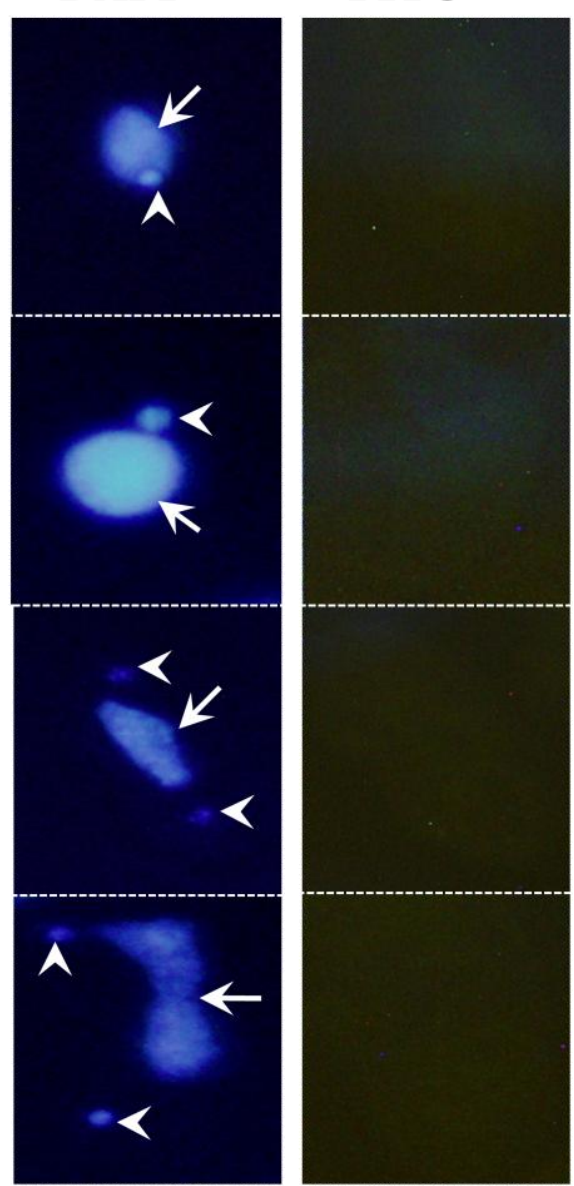

Figure 12: Indirect immunofluorescence of Nrp1-GFP (A) and untagged cells (B) using anti-GFP antibody. Nrp1 localization is temporally correlated with mitosis in logarithmically growing T.thermophila. Cells were stained with DNA-specific dye DAPI to capture the nuclei orientation whereas anti-GFP antibody was used to identify Nrp1 localization. Arrow heads denote the position of MIC whereas open arrows indicate the MAC location. 


\section{2- Characterization of HIRA in T. thermophila}

\subsection{2- Hira is evolutionarily conserved}

The evolutionarily conserved Hira family of histone chaperones are known to function in RI chromatin assembly pathway (Green et al. 2005) . These proteins possess several WD40 repeats which are 40 amino acid long structural motifs (Balaji et al. 2009; Xu and Min 2011). In T. thermophila genome TTHERM_00046490 has been identified as putative Hira homolog. In order to determine and assess the arrangement of WD40 motifs the primary amino acid sequences of Hira $^{\mathrm{Tt}}$ as well as human Hira and budding yeast Hir1 proteins were submitted to the SMART web server (see methods). As indicated in figure 13A, similar to both human and $S$. cerevisiae which respectively possess eight and six WD40 repeats, Hira ${ }^{\text {Tt }}$ also has at least five predicted WD40 motifs. In addition, Hira signature domain PF07569 (HIRA) is also present in $\mathrm{Hira}^{\mathrm{Tt}}$ as predicted by PFAM domain analysis (Figure 13-A). These studies suggest that $\mathrm{Hira}^{\mathrm{Tt}}$ is well conserved and shares common domain architecture with other well characterized homologs.

Hira family proteins are also known to have a B-domain which resides outside of the WD40 repeats and is responsible for the physical interaction with Asf1 (Tang et al. 2006). The presence of B-domain in Hira family proteins has predominantly been described only in higher eukaryotes ranging from fungi to mammals. However, MSA analysis shown in figure 13 indicated the presence of a putative B-domain in Hira $^{\text {Tt }}$ as well, suggesting an ancient origin of Hira-Asf1 interaction (Figure 13-B). Notably, the minimum Hira peptide required for Asfla binding in humans is highly conserved (block box in Figure 13-B). More remarkably, the B-domain residues that have been shown to make the contact with Asf1a have nearly remained unchanged over the course of evolution (Tang et al. 2006) (see "stars" 
in Figure 13-B). These observations suggest that Hira ${ }^{\mathrm{Tt}}$ might have a physical interaction with Asf $1^{\mathrm{Tt}}$.
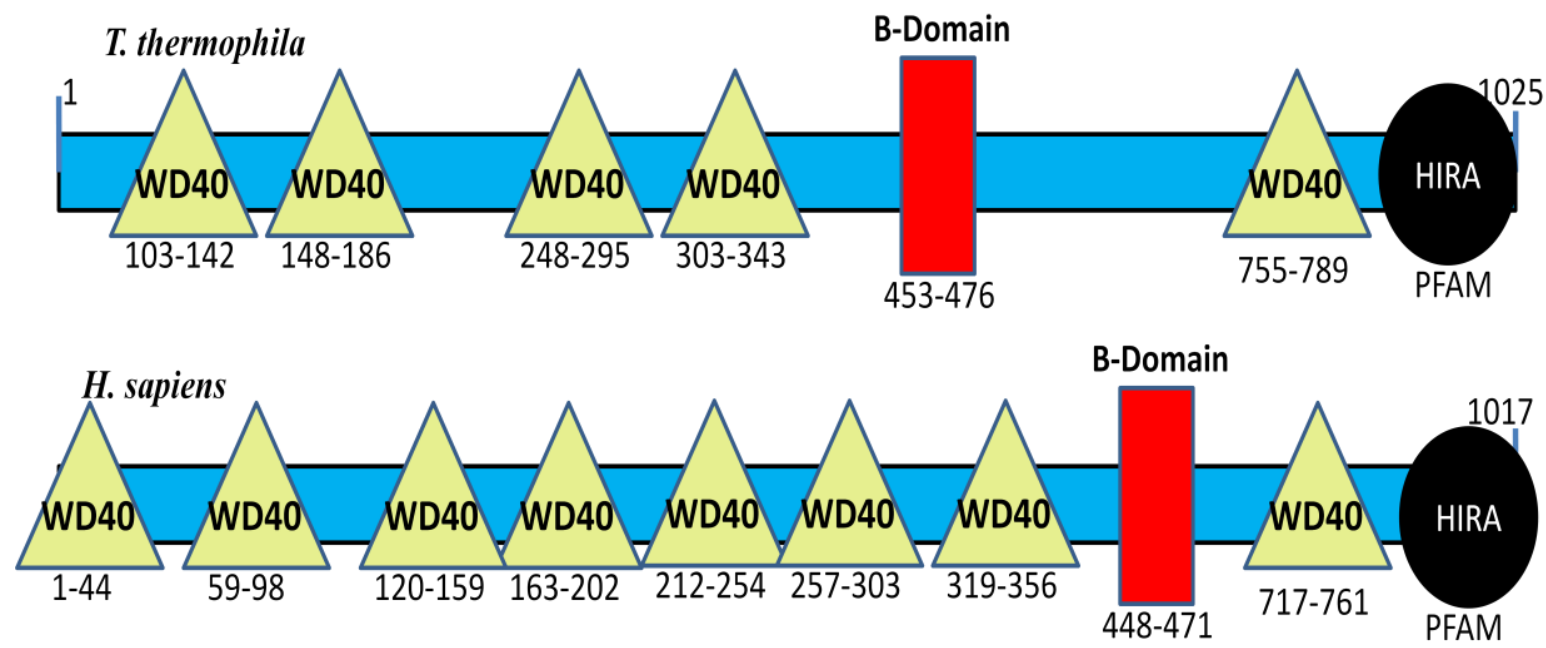

S. cerevisiae

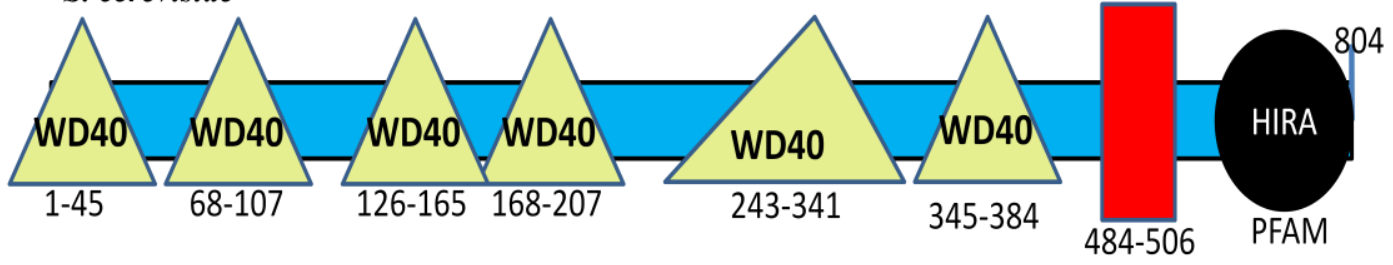

B
H. sapiens/1-26
M. musculus/1-26
S. cerevisiae/1-24
S. pombe/1-24
T. thermophila/1-26

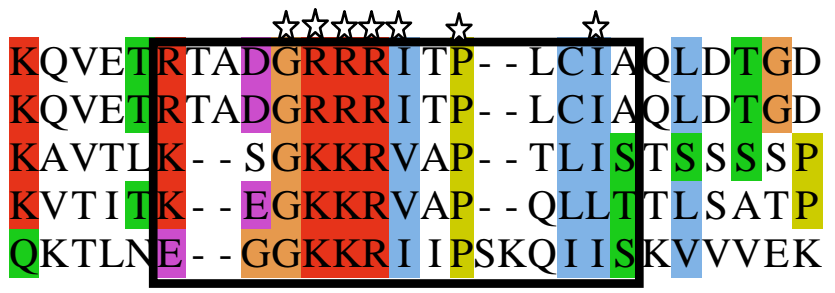

Figure 13: Structural comparison of various Hira homologs. A: The figure is based on PFAM and SMART domain analyses. The number and positions of the amino acid residues comprising each domain are also indicated. B: Multiple sequence alignments of HirA Bdomain across various lineages. The black box indicated the minimum human peptide required for Asf1a binding whereas stars signify the residues that make the initial contact. 
The WD40 repeats are among the most abundant domains across eukaryotic proteomes (Hennig et al. 2005). These motifs have been shown to be involved in a variety of cellular processes including signal transduction, cell cycle control, apoptosis, chromatin related processes and transcription regulation (reviewed in $\mathrm{Xu}$ and Min 2011). Several studies have shown that WD40 motifs adopt a 7 - or 8 - bladed $\beta$-propeller structure that is responsible for a plethora of protein-protein interactions (reviewed in Xu and Min 2011). The Hira proteins have been described to contain $\mathrm{N}$ - and $\mathrm{C}$-terminal 7-bladed $\beta$-propellers (see PDB entry 2YMU; Chaudhuri I. and Zeth K., unpublished) which are separated by a Bdomain linker region that interacts with Asf1. In order to investigate the structural features, the primary amino acid sequence of $\mathrm{Hira}^{\mathrm{Tt}}$ was submitted to the I-TASSER for a three dimensional structure prediction (Figure 14). The $\mathrm{Hira}^{\mathrm{Tt}}$ is predominantly composed of $\beta$ strands $(\sim 30 \%)$ and disordered regions (40\%) whereas alpha helices only make up to $12 \%$ of the whole protein. The $\mathrm{N}$ - terminal of the protein is predicted to form a classical 7-bladed $\beta$ propeller encompassing approximately first 350 residues (Figure $14 \mathrm{~B}$ ). The C-terminus however is not predicted to form a $\beta$-propeller and contains several alpha helices facing opposite to each other. The B-domain is found within the middle region $(\sim 350-790$ residue positions) which separates the $\mathrm{N}$ - and $\mathrm{C}$ - termini of the protein. This middle region is mainly disordered with a few $\beta$ sheets also found within. Remarkable, the overall packaging of this region closely resembles with the $\beta$-propeller structure (Figure $14 \mathrm{C}$ ). This packaging presumably is stabilized by the $11 \beta$ sheets that are found in this resgion. Taken together, Hira $^{\mathrm{Tt}}$ is predicted to be somewhat structurally divergent relative to its well characterized counterparts in other organisms (see PDB 2YMU). The lack of C-terminal $\beta$-propeller and the presence of a middle $\beta$-propeller-like structure may represent lineage specific alterations that might have important roles distinct from those described in higher eukaryotes. 


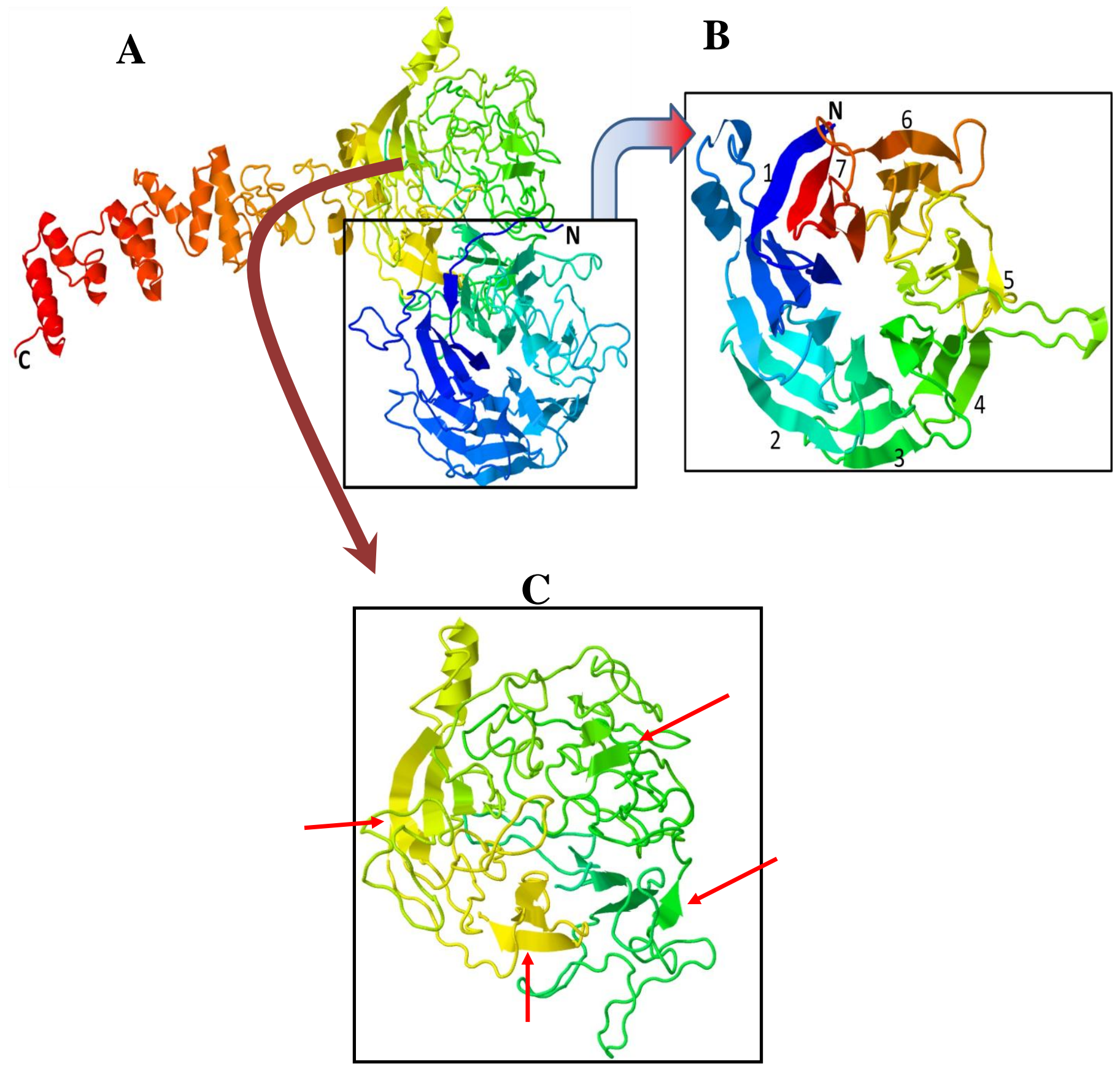

Figure 14: Predicted tertiary structure of T. thermophila Hir1 protein. A- Overall three dimensional structure of the whole protein. The ribbon structure is coloured in rainbow scheme with dark blue representing $\mathrm{N}$ - terminal whereas red indicating the $\mathrm{C}$-terminal. BClosed view of N-terminal $\beta$-propeller. Seven blades are number from 1-7. C- Closed view of middle region which adopts an overall $\beta$-propeller-like packaging. Red arrows denote the position of beta sheets found within this region. 


\subsection{3- Proteomic analysis of T. thermophila Hira}

In order to examine the protein-protein interactions of Hira, I generated a stable $T$. thermophila cell line expressing Hira $^{\mathrm{Tt}}$ with a C-terminal FZZ epitope tag from the MAC locus. Following phenotypic assortment in the MAC, WCEs from the vegetative Hira ${ }^{\mathrm{Tt}}$-FZZ and un-tagged wild type cell lines were prepared. Western blot analysis of these WCEs was performed using anti-FLAG antibody to assess the expression of tagged cell lines. A clear signal was observed around $135 \mathrm{kDa}$ in the $\mathrm{Hira}^{\mathrm{Tt}}$-FZZ lane but not in the wild type (Figure 15) indicating successful generation of desired cell line.

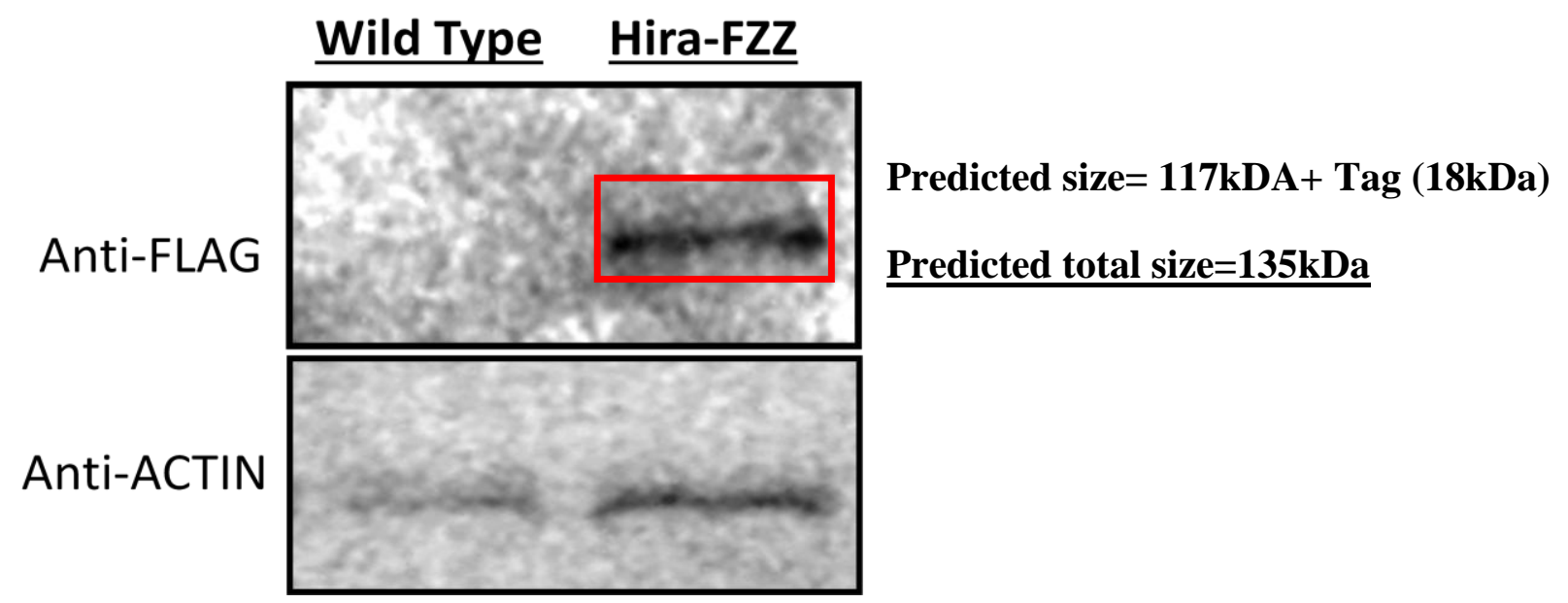

Figure 15: Western blot of Hira-FZZ indicating the successful expression of the tagged protein. Top panel was probed with anti-FLAG antibody which showed a strong signal in the lane corresponding to Hir1-FZZ whereas no signal was detected in the wild type control. The band appeared within the reasonable range of the fusion protein predicted size $(\sim 135 \mathrm{kDa})$. The lower panel was probed with anti-actin antibody as a loading control.

One step affinity purification procedure was used to purify $\mathrm{Hira}^{\mathrm{Tt}}$-FZZ. I employed one step AP in order to increase the likelihood of capturing transient interactions that might be lost using two step affinity purification procedure (Fillingham et al. unpublished observations). The recovery of the purified proteins was assessed using Western blotting and strong signal was observed in the Hira ${ }^{\mathrm{Tt}}$-FZZ lanes when probed with anti-FLAG antibody (Figure 16). Note, in one step purification procedure, the TEV-cleavage step is eliminated and therefore the purified Hira $^{\mathrm{Tt}}$-FZZ remains equal in size with that of input. Subsequently, 
purified material was analysed by gel-free LC-MS/MS to detect any co-purifying proteins. The mass spectrometry data from two sets of Hira ${ }^{\mathrm{Tt}}$-FZZ affinity purifications was subjected to SAINT analysis. The SAINT analysis identified the successful recovery of the bait Hira ${ }^{\mathrm{Tt}}$ (TTHERM_00046490) protein with 27 spectral counts however no other significant proteins were identified as prey. I am currently attempting additional purifications of Hira ${ }^{\mathrm{Tt}}{ }_{-} \mathrm{FZZ}$ from whole cell extracts made from this cell line.

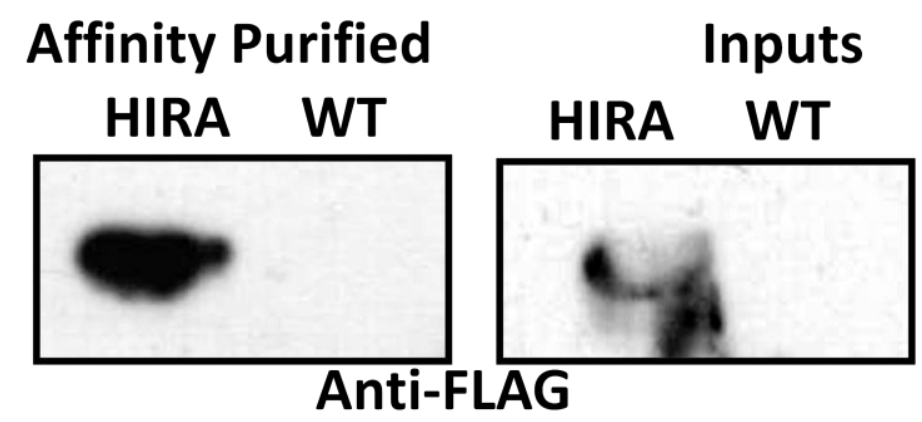

Figure 16: Western blots probed with anti-FLAG antibody indicating the successful recovery of affinity purified Hira ${ }^{\mathrm{Tt}}$. An intense signal in the affinity purified Hira ${ }^{\mathrm{Tt}}$ lane signifies huge amount of protein present in the sample. No signal was detected in any of the wild type lanes. Note: One-step purification does not result in smaller size of the purified protein due Tev cleavage step elimination.

Several studies have shown that network analysis of gene expression data can be used to identify related genes functioning in the same pathway or part of same protein complex. In order to gain additional insights into $\mathrm{Hira}^{\mathrm{Tt}}$ functions, I used publically available gene network data ( $\mathrm{Z}$ score threshold 4.5$)$ based on expression profiles and represented it as an undirected network. The final network contained 350 nodes (Figure 17-A). The histone H3-H4 chaperones $\mathrm{Asf1}^{\mathrm{Tt}}$ and Nrp1 both are linked to Hira ${ }^{\mathrm{Tt}}$ in this network consistent with previous studies in yeast and humans (Tagami et al. 2004; Tang et al. 2006) indicating that these proteins likely function together in a chromatin assembly pathway despite the fact that in $T$. thermophila Hira does not co-purify with Asf1 (see above). In addition, subunits of $T$. 
thermophila putative FACT complex, Spt16 (TTHERM_00283330) and Pob3 (TTHERM_00049080), chromosomal maintenance proteins SMC1 (TTHERM_01048090) and SMC4 (TTHERM_00446400) as well as a putative MCM4 (TTHERM_00277550) which is a member of MCM complex responsible for DNA replication, are linked to Hira ${ }^{\mathrm{Tt}}$. These observations suggest that $\mathrm{Hira}^{\mathrm{Tt}}$ is a multi-functional protein involved in chromatinrelated processes. To investigate whether the functional links found in Hira ${ }^{\mathrm{Tt}}$ network are conserved in higher eukaryotes, I extracted human Hira gene network. The network shown in figure 17-B has 100 nodes and is based on physical interaction, co-localization and coexpression data available through GeneMania web server (Warde-Farley et al. 2010 and references therein). Interestingly, in addition to Ubinuclein and Cabin1 which are subunits of Hira complex, Asf1a, Asf1b, subunits of FACT complex (SPT16 and POB3) as well as SMC5 are also functionally linked to Hira. Interestingly, in budding yeast Hir proteins genetically interact with the FACT complex (Formosa et al. 2002). The overall parallelism found between two networks suggests that Hira family of histone chaperones is functionally conserved. 


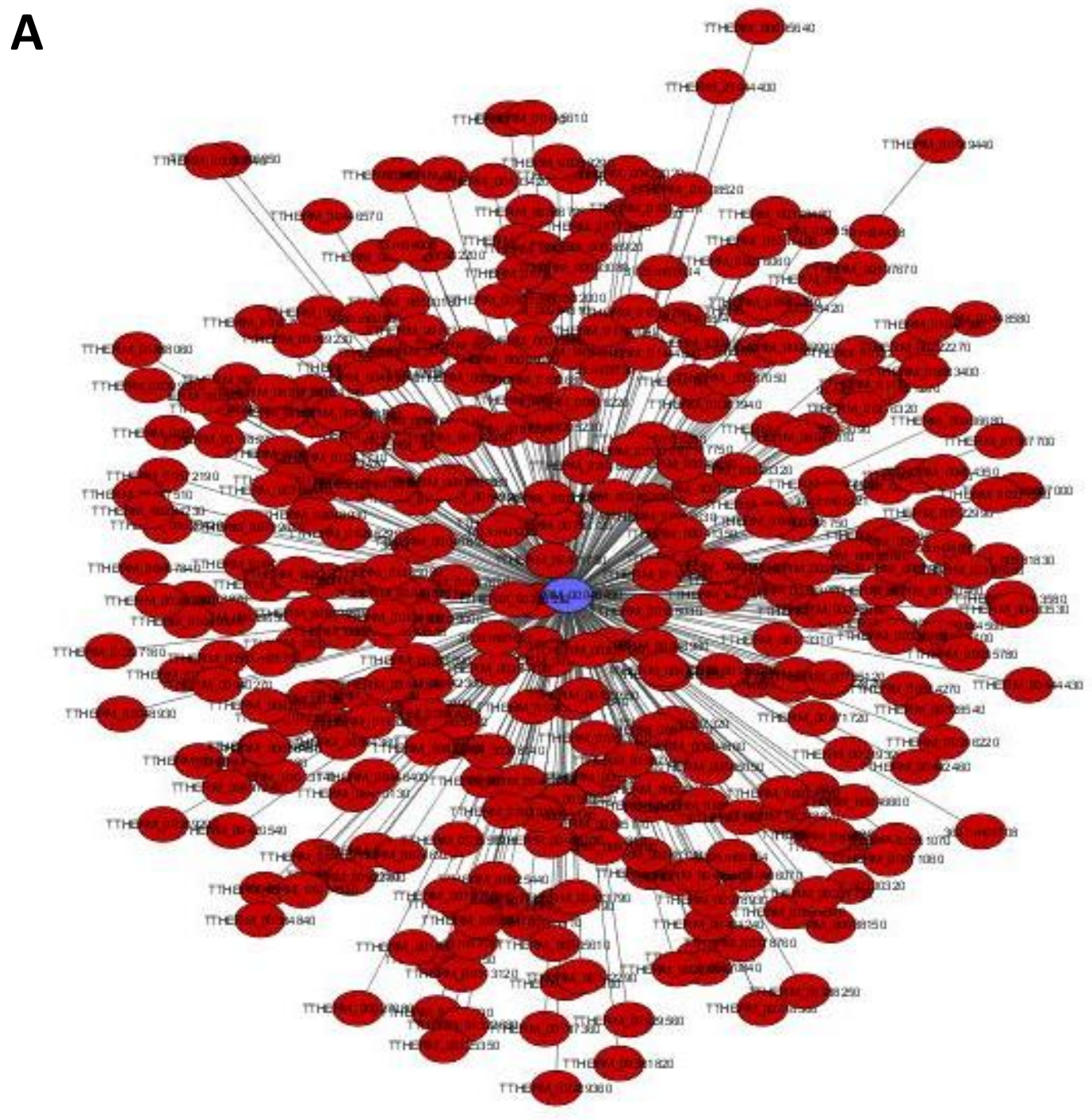




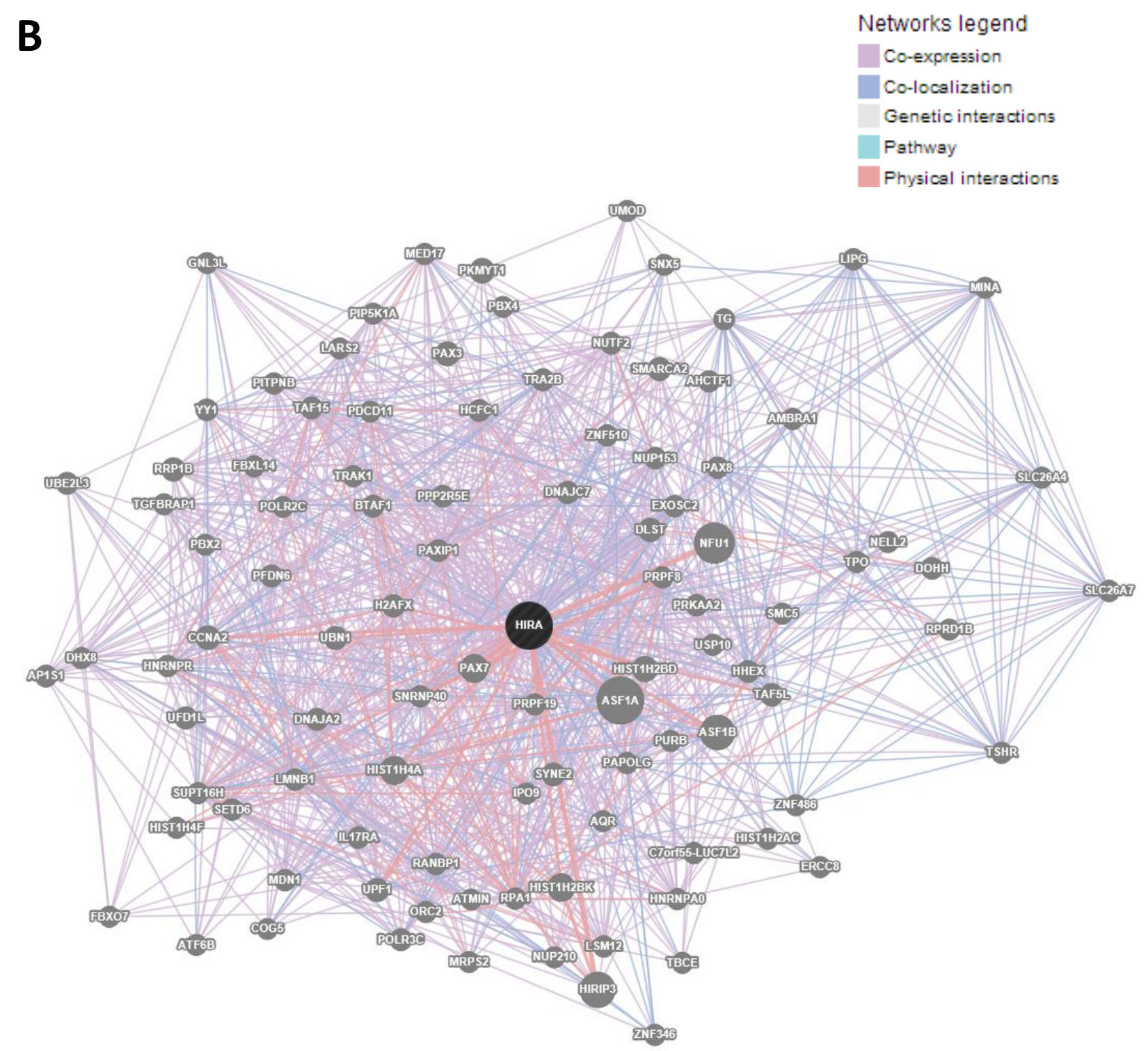

Figure 17: Gene networks Hira homologs in $T$. thermophila and $H$. sapiens. A- T. thermophila Hira gene network based on expression profiles. The network was downloaded from $T$. thermophila functional database website and represented as an un-directed network. It has 350 nodes and HIRA is coloured in blue whereas its first neighbours are shown in red. B- Gene network of $H$. sapiens constructed using GeneMania web server. The network has 100 nodes and is based on physical interactions, co-localization and co-expression data. The color legend is provided. 


\subsection{4- Hira ${ }^{\mathrm{Tt}}$ localizes to MAC and MIC}

In humans, Hira has been demonstrated to function in the deposition of transcriptionassociated H3.3 variant onto chromatin in a RI manner. T. thermophila has four H3 genes including HHT2 (TTHERM_00189180), HHT3 (TTHERM_00016170), HHT4 (TTHERM_00016200) and a centromeric histone CNA1 (TTHERM_00146340). The major histone $\mathrm{H} 3$ is encoded by $H H T 2$ whereas $H H T 3$ and $H H T 4$ respectively encode minor $\mathrm{H} 3.3$ and H3.4 variants. In T. thermophila the major $\mathrm{H} 3$ has been shown to localize to both MAC and MIC whereas H3.3 predominantly is found in MAC chromatin (Cui et al. 2006).

In order investigate the functional roles of $\mathrm{Hira}^{\mathrm{Tt}}{ }^{\mathrm{T}}$ I carried out an indirect IF using anti-FLAG primary antibody in growing cells (Figure 18). The signal was observed only in the Hira ${ }^{\mathrm{Tt}}$-FZZ samples and not in the wild type un-tagged control cells. As apparent from the figure 18, like H3.3, Hira $^{\mathrm{Tt}}{ }_{-}$-FZZ localizes predominantly to the MAC and also to the MIC. The predominant MAC localization of Hira ${ }^{\mathrm{Tt}}-\mathrm{FZZ}$ is consistent with its known role in transcription associated RI chromatin assembly. The signal which is also persistent in the MIC after its division suggests that $\mathrm{Hira}^{\mathrm{Tt}}$ might be involved in additional functions with respect to silent MIC chromatin maintenance (see discussion)(Figure $18 \mathrm{C}$ ). 


\section{HIRA-FZZ}

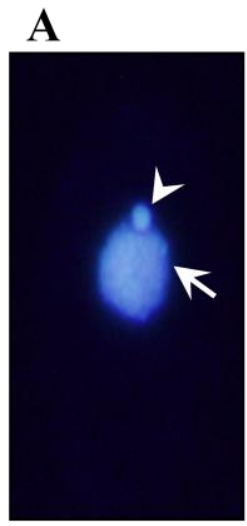

DAPI

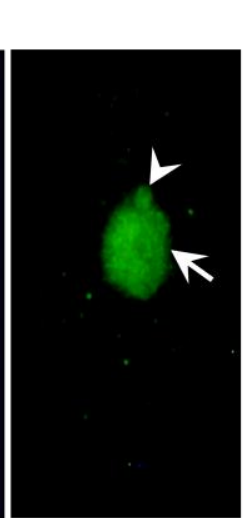

FITC

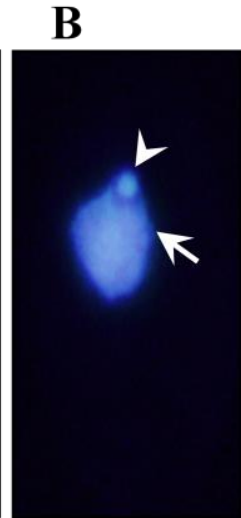

DAPI

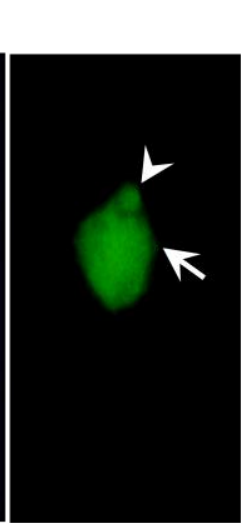

FITC
C

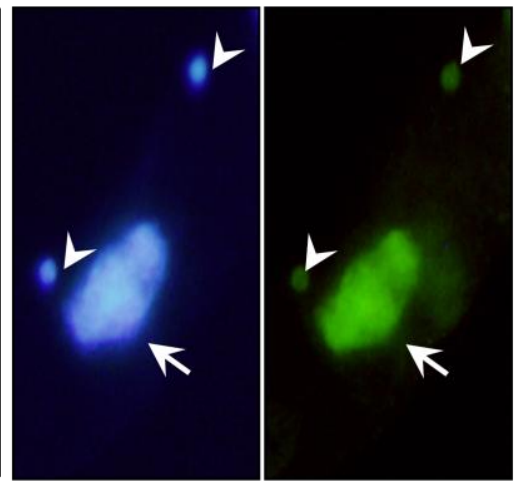

DAPI

FITC

\section{Wild Type}

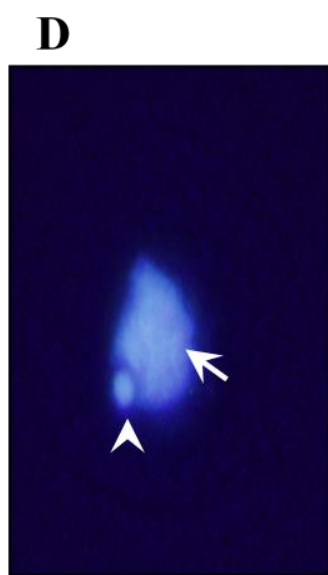

DAPI

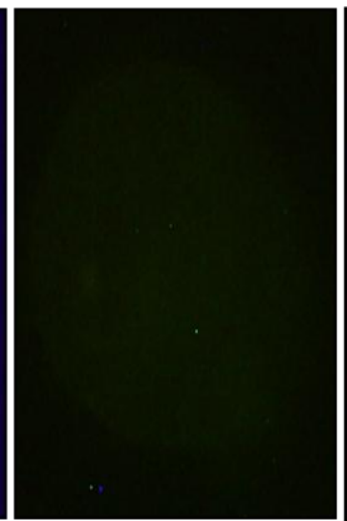

FITC

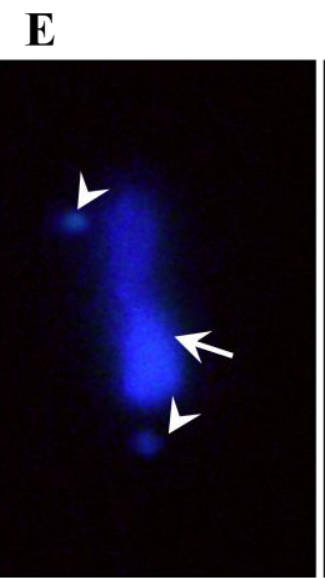

DAPI

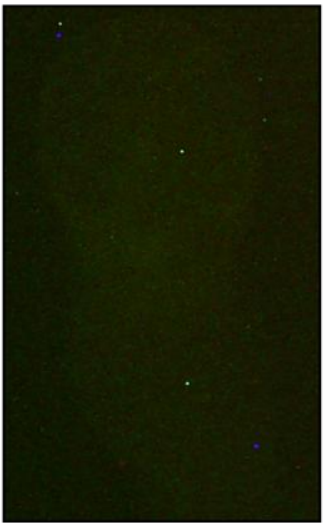

FITC

Figure 18: Indirect immunofluorescence of Hira ${ }^{\mathrm{Tt}}$-FZZ (A-C) and untagged cells (D-E) using anti-FLAG antibody. Hira localizes to both MAC and MIC while signal is relatively faint in the MIC. Cells were stained with DNA-specific dye DAPI to capture the nuclei orientation. Arrow heads denote the position of MIC whereas open arrows indicate the MAC location. 


\section{3- Functional characterization of Cac2 subunit of CAF1 ${ }^{\mathrm{Tt}}$ complex}

Cac2 subunit of Caf1 complex shares several structural and functional features with its ancient paralog Hira. Similar to Hira, several WD40-repeats and the B-domain which is responsible for Asf1 interaction also exists in Cac2. However in contract with Hira which has two $\beta$-propellers, Cac2 has been suggested to have lost its C-terminal $\beta$-propeller region (Balaji et al. 2009) .

To study the function of CAF1 complex, I initiated my analysis with BLASTP searches against $T$. thermophila MAC genome using budding yeast Cac2 as query, and identified TTHERM_00219420 as a putative Cac2 subunit. Domain analysis of Cac2 ${ }^{\mathrm{Tt}}$ indicates that it has six WD40 repeats (Figure 19) which is similar to human and budding yeast possessing seven and six WD40 repeats, respectively. In addition, MSA analysis

predicts that $\mathrm{Cac}_{2}{ }^{\mathrm{Tt}}$ also has a putative $\mathrm{B}$-domain like region suggesting that $\mathrm{Cac} 2^{\mathrm{Tt}}-\mathrm{Asf} 1^{\mathrm{Tt}}$ interaction might be conserved in this divergent organism. However, despite the overall homology the putative $\mathrm{B}$-domain in $\mathrm{Ca} 2^{\mathrm{Tt}}$ has a noticeable difference, i.e. the arginine (R) residues important for Asf1 interaction has been substituted with lysines (K) (See Figure 20). Structural prediction of $\mathrm{Cac}^{\mathrm{Tt}}$ (Figure 21) indicates that it forms two $\beta$-propellers. These two $\beta$-propellers can be easily divided into $\mathrm{N}$-terminal and $\mathrm{C}$-terminal regions. The $\mathrm{N}$-terminal $\beta$ propeller encompasses first 275 residues whereas the C-terminal $\beta$-propeller is composed of remaining 270 residues. The first 16 residues adopt an extended topology and reside near the very last residues of the protein where the putative B-domain is found. Interestingly both $\beta$ propellers of $\mathrm{Cac} 2^{\mathrm{Tt}}$ are predicted to be composed of 5- blades (N-terminal: 1-5; C-terminal: 6-10 in Figure 21) in contrast to Hira which has been suggested to carry 7-bladed $\beta$ propellers. 

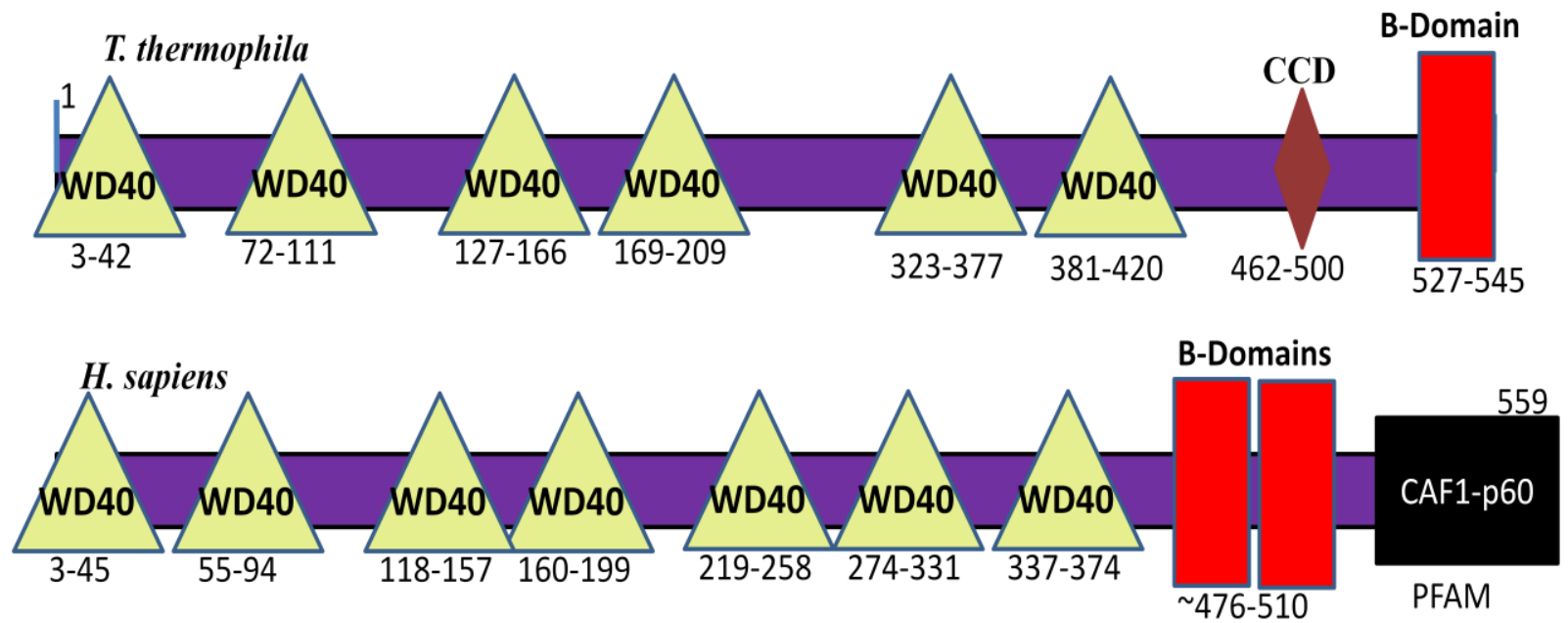

S. cerevisiae

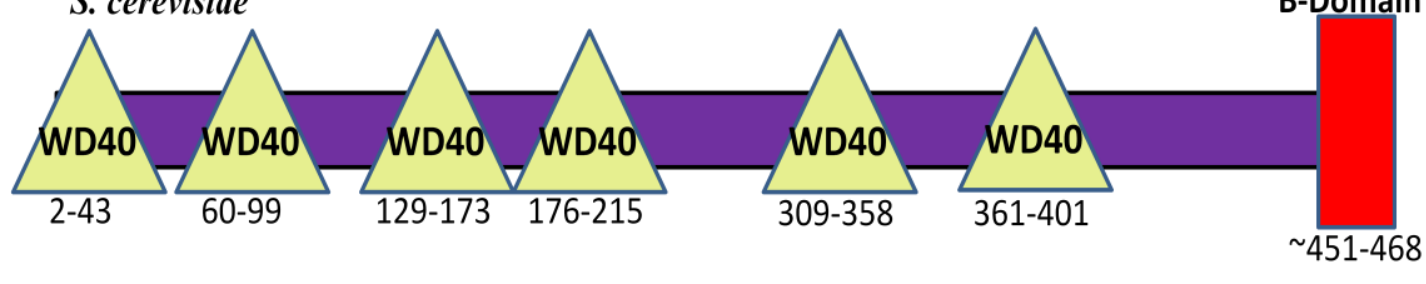

Figure 19: Structural comparison of various Cac2 homologs. The figure is based on SMART domain analysis. The number and positions of the amino acid residues comprising each domain are also indicated.

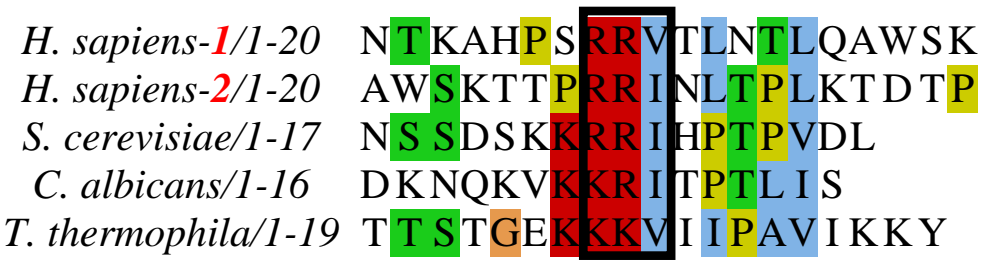

Figure 20: Multiple sequence alignments of Cac2 B-domain across various lineages. The black box indicated predicted residues required for Asf1 binding. 


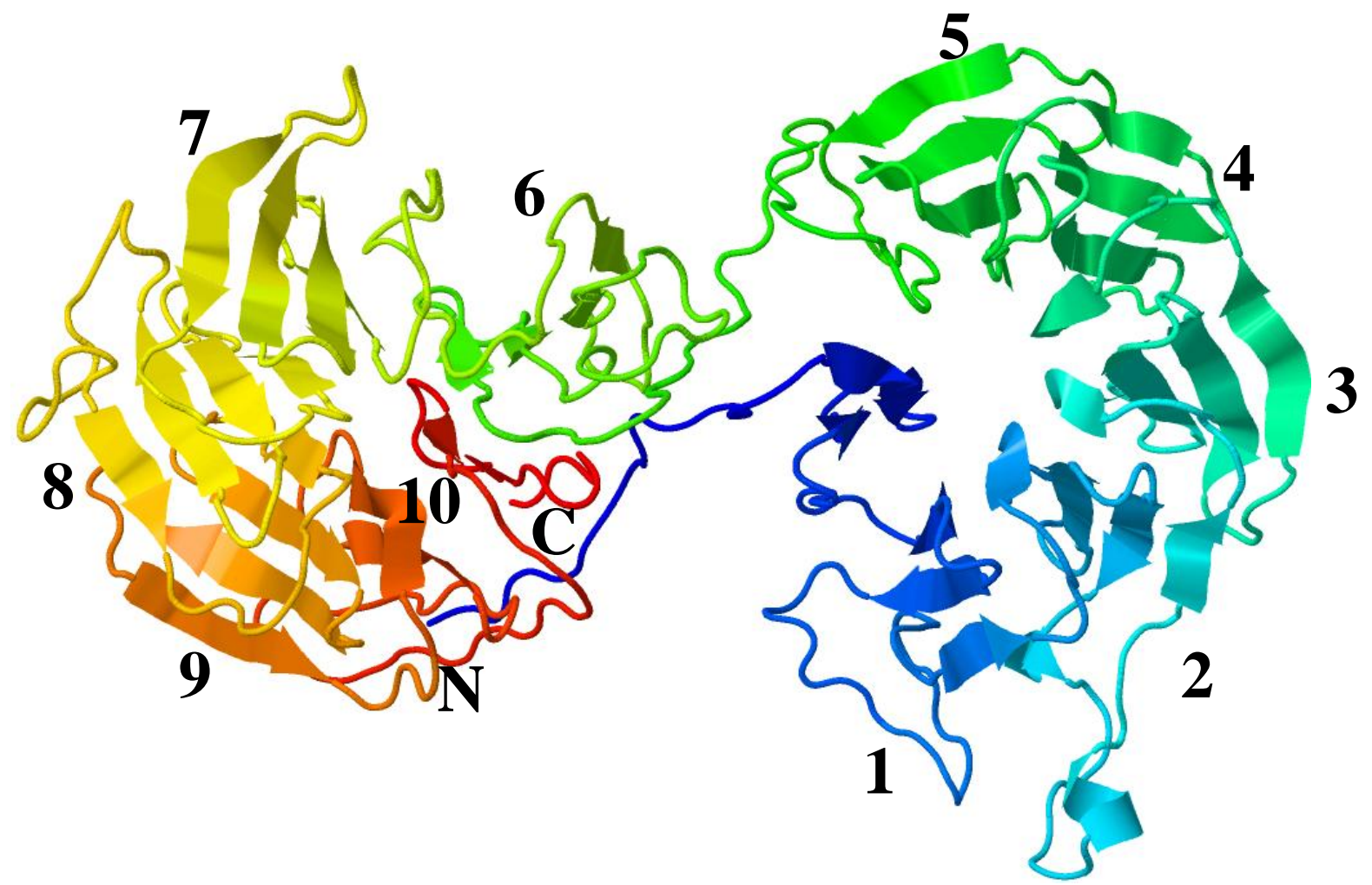

Figure 21: Predicted tertiary structure of $T$. thermophila Cac2 protein. Overall three dimensional structure of whole protein: The ribbon structure is coloured in rainbow scheme with dark blue representing $\mathrm{N}$ - terminal whereas red indicating the $\mathrm{C}$-terminal. Different blades of the two $\beta$-propellers are numbered from 1-10. 


\subsection{2- CAF1 ${ }^{\mathrm{Tt}}$ is conserved}

To investigate the functional aspects of $\mathrm{Caf} 1^{\mathrm{Tt}}$ I generated stable $\mathrm{Cac} 2^{\mathrm{Tt}}$ cell lines expressing C-terminal FZZ epitope tag from the native chromosomal loci. After the completion of phenotypic assortment, the WCEs were prepared from the growing cells carrying either $\mathrm{Cac} 2^{\mathrm{Tt}}$-FZZ or untagged wild type. Western blot using anti-FLAG antibody indicated a strong signal of approximately $78 \mathrm{kDa}$ in the $\mathrm{Cac} 2^{\mathrm{Tt}}-\mathrm{FZZ}$ lanes and not in the wild type (Figure 22). These results indicated the successful expression of the tagged proteins. Subsequently, pellets from the growing cells were prepared and TAP was carried out in order to purify $\mathrm{Cac} 2^{\mathrm{Tt}}-\mathrm{FZZ}$ along with interacting proteins. The successful recovery of the purified Cac2 ${ }^{\mathrm{Tt}}$-FZZ was assessed by Western blotting. Strong signals were observed in the Cac2 ${ }^{\mathrm{Tt}}$ FZZ input and TAP lanes but not in the wild type (note the size difference in Figure 23 due to Tev cleavage in TAP lanes). The TAP experiment was repeated twice in order to provide experimental replicas necessary for statistical analysis.

As indicated in the Table 3, SAINT analysis of the AP-MS data from vegetative $T$. thermophila cells revealed five putative $\mathrm{Cac} 2^{\mathrm{Tt}}$ interacting partners with $\mathrm{AvgP}>0.9$. The identified $\mathrm{Cac}_{2}{ }^{\mathrm{Tt}}$ interacting proteins were used as queries to search the NCBI non-redundant database. In addition, SMART domain analysis was also carried out to investigate the structural features. The gene TTHERM_00309890 encodes a predicted 1027 amino acid protein which carries a Cac1 signature PFAM domain CAF1A (PFAM ID: PF12253) whereas TTHERM_00688660 shares significant homology with Cac3 as assessed by BLASTp searches (score $5 \mathrm{e}^{-150}$ ). In addition, PFAM domain analysis indicates that it also possesses Cac3 PFAM signature domain PF12265. Together, these results suggest that subunits of T. thermophila putative $\mathrm{CAF}^{\mathrm{Tt}}$ complex have co-purified with $\mathrm{Cac}^{\mathrm{Tt}}$-FZZ and that I have identified a bona-fide ciliate CAF1 complex. In addition, the other three Cac2-co- 
purifying gene Ids TTHERM_01345800, TTHERM_00780530 and TTHERM_01000180

(Table 3) shared homology with the subunit of serine/threonine selective protein kinase 'casein kinase II (CKII) complex' (score $2 \mathrm{e}^{-129}$ ). The CKII complex is conserved in eukaryotes and in humans and yeast is composed of two regulatory beta subunits (CKB) and two catalytic alpha (CKA) subunits (Meggio and Pinna 2003). The proteins encoded by TTHERM_01345800 and TTHERM_01000180 share homology with the budding yeast CKA1 and CKA2 subunits, respectively, whereas TTHERM_00780530 is homologous to the two regulatory beta subunits. Because only one beta subunit of $T$. thermophila putative CKII complex co-purified therefore I carried out BLAST searches against MAC genome to identify any additional putative beta subunits. However, despite extensive searches I was unable to predict the existence of any additional beta subunits. This suggests that $T$. thermophila putative CKII complex only has one beta unit as the sole homolog of two regulatory subunits found in other organisms. Confirmation awaits successful reciprocal tagging and purification of CKII (ongoing project).

Predicted size Cac2=60kDa FZZ predicted size $=18 \mathrm{kDa}$ Total fusion protein $=78 \mathrm{kDa}$

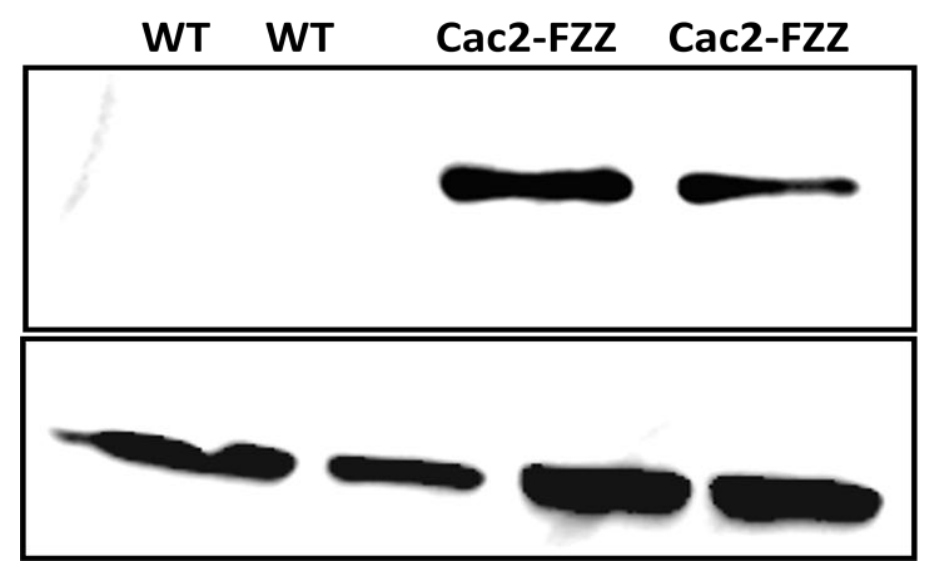

Figure 22: Western blot of $\mathrm{Cac2}^{\mathrm{Tt}}-\mathrm{FZZ}$ indicating the successful expression of the tagged protein in growing cells. Top panel was probed with anti-FLAG antibody which showed a strong signal in the lane corresponding to $\mathrm{Cac} 2^{\mathrm{Tt}}-\mathrm{FZZ}$ whereas no signal was detected in the wild type control. The band appeared within the reasonable range of the fusion protein predicted size $(\sim 78 \mathrm{kDa})$. The lower panel was probed with anti-actin antibody as a loading control. 


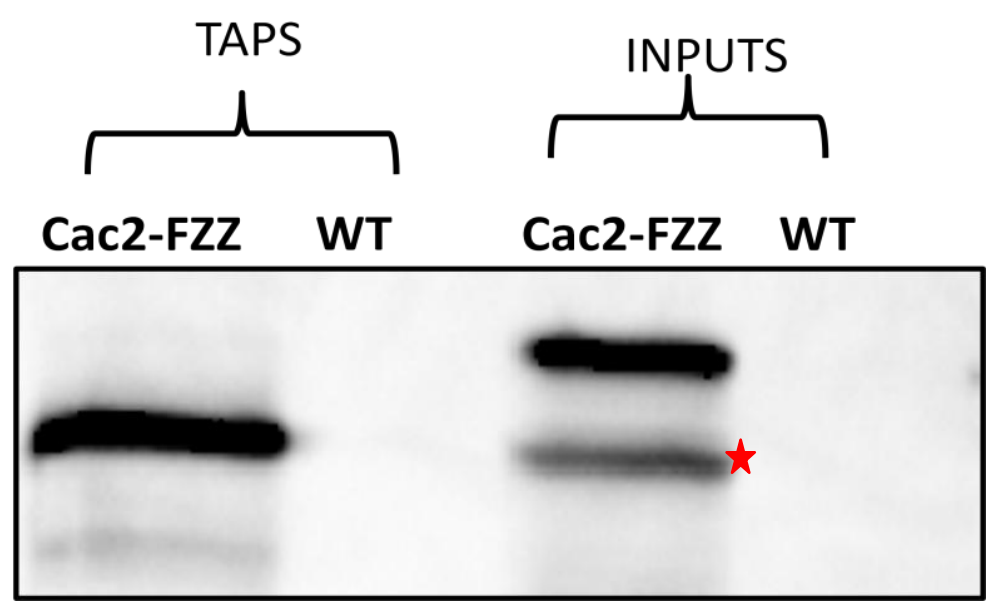

Figure 23: Successful recovery of purified $\mathrm{Cac2}^{\mathrm{Tt}}-\mathrm{FZZ}$ protein detected by Western blot. The blot was probed with anti-FLAG antibody to detect the signals in the Cac2 ${ }^{\mathrm{Tt}}$ input and TAP lanes. In contrast no signal was detected in the wild type lanes. Note, the size of $\mathrm{Cac} 2^{\mathrm{Tt}}$ in the TAP lane is smaller than the input lane indicating the removal of protein A part of the tag. The red star indicates the proteolytic degradation.

Table 3: AP-MS data filtered using SAINT analysis. The AvgP represents the overall probability of individual experimental replicates

\begin{tabular}{|c|c|c|c|c|c|}
\hline Bait & Prey & $\begin{array}{c}\text { Gene ID } \\
\text { Tetrahymena }\end{array}$ & $\begin{array}{l}\text { Spect } \\
\text { Samples } \\
\end{array}$ & $\begin{array}{l}\text { ts } \\
\text { ntrol } \\
\end{array}$ & $\operatorname{Avg} P$ \\
\hline $\mathrm{Cac} 2^{\mathrm{Tt}}$ & $\mathrm{Cac} 2^{\mathrm{Tt}}$ & TTHERM_00219420 & $62 / 36$ & $0 / 0 / 0$ & - \\
\hline $\mathrm{Cac} 2^{\mathrm{Tt}}$ & $\mathrm{Cac}^{\mathrm{Tt}}$ & TTHERM_00309890 & $77 / 69$ & $0 / 0 / 0$ & 1 \\
\hline $\mathrm{Cac} 2^{\mathrm{Tt}}$ & $\mathrm{Cac} 3^{\mathrm{Tt}}$ & TTHERM_00688660 & $76 / 40$ & $0 / 0 / 0$ & 1 \\
\hline $\mathrm{Cac} 2^{\mathrm{Tt}}$ & $\mathrm{CKA} 1^{\mathrm{Tt}}$ & TTHERM_01345800 & $17 / 4$ & $0 / 0 / 0$ & 1 \\
\hline $\mathrm{Cac} 2^{\mathrm{Tt}}$ & $\mathrm{CKB} 1^{\mathrm{Tt}}$ & TTHERM_00780530 & $14 / 3$ & $0 / 0 / 0$ & 0.99 \\
\hline $\mathrm{Cac} 2^{\mathrm{Tt}}$ & $\mathrm{CKA} 2^{\mathrm{Tt}}$ & TTHERM_01000180 & $7 / 3$ & $0 / 0 / 0$ & 0.99 \\
\hline
\end{tabular}




\subsection{3- T. thermophila Cac2 localizes predominantly to MIC}

To gain insights into $\mathrm{Cac} 2^{\mathrm{Tt}}$ function I used indirect IF using anti-FLAG primary antibody in $\mathrm{Cac}^{\mathrm{Tt}}$-FZZ growing cells. As apparent from figure 24 , Cac2 ${ }^{\mathrm{Tt}}$-FZZ localizes to both MAC and MIC, however more intense signal is observed in the MIC. This pattern of localizations closely resembles to that of $T$. thermophila Asf1 as we previously demonstrated in (Garg et al. 2013). In order to directly compare the localization patterns, I acquired Asf ${ }^{\mathrm{Tt}}$-FZZ strains and performed an indirect immunofluoresence on growing Asf ${ }^{\mathrm{Tt}}$-FZZ cells. My results consistently indicated a similar pattern of localization for both $\mathrm{Cac}^{2}{ }^{\mathrm{Tt}}-\mathrm{FZZ}$ and Asf $1^{\mathrm{Tt}}$-FZZ being found in both MAC and MIC, with more intense signal visible in the MIC (Figure 25). The similar pattern of localization suggests that Asf $1^{\mathrm{Tt}}$ and $\mathrm{Cac} 2^{\mathrm{Tt}}$ might be functionally linked to each other consistent with their demonstrated interaction in other organisms as well as presence of Asf1 interacting B-domain in Cac2 ${ }^{\mathrm{Tt}}$ (See above). 
DAPI
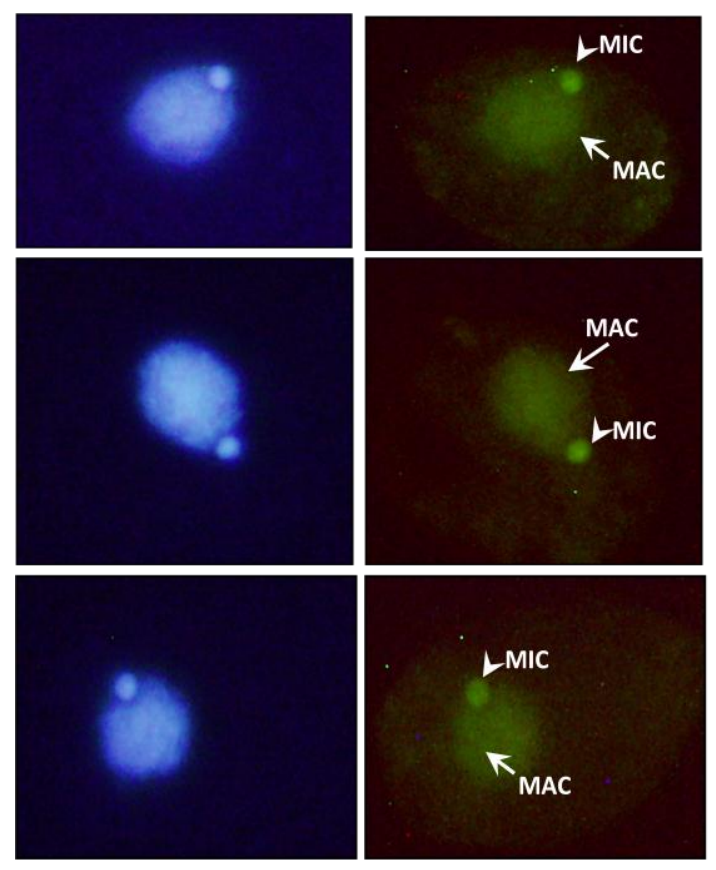

\section{Cac2-FZZ}

Figure 24: Indirect immunofluorescence of $\mathrm{Cac}^{\mathrm{Tt}}{ }_{-}^{\mathrm{FZZ}}$ and untagged cells using antiFLAG antibody. Cac2 ${ }^{\mathrm{Tt}}$ localizes to both MAC and MIC while the signal is more intense in the MIC. Cells were stained with DNA-specific dye DAPI to capture the nuclei orientation. Arrow heads denote the position of MIC whereas open arrows indicate the MAC location.
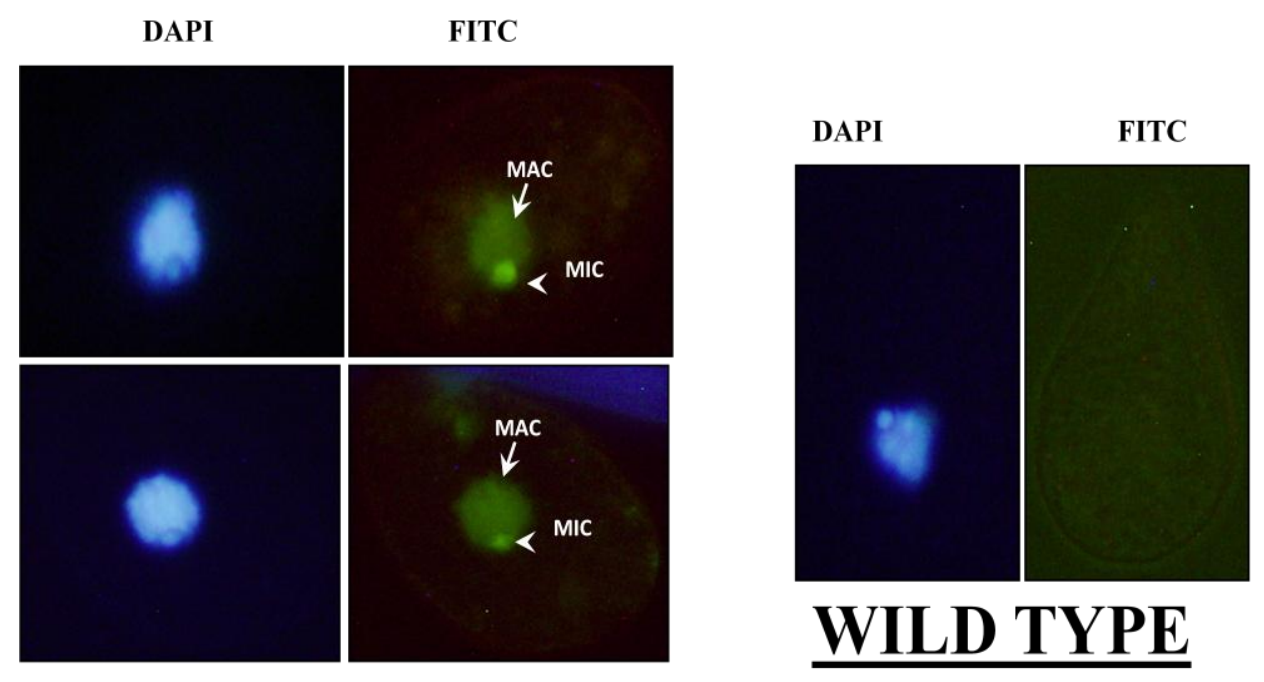

\section{$\underline{\text { Asf1-FZZ }}$}

Figure 25: Indirect immunofluorescence of Asf1 ${ }^{\mathrm{Tt}}$-FZZ and untagged cells using antiFLAG antibody. Asf $1^{\mathrm{Tt}}$ localizes to both MAC and MIC while the signal is more intense in the MIC. Cells were stained with DNA-specific dye DAPI to capture the nuclei orientation. Arrow heads denote the position of MIC whereas open arrows indicate the MAC location. 


\section{4- Functional characterization of Hat1 in T. thermophila}

Newly synthesized histones have been found to be deposited onto chromatin with specific pattern of PTMs (Allis et al. 1985). Several early studies showed that in $T$. thermophila, newly synthesized histone $\mathrm{H} 4$ are heavily diacetylated at $\mathrm{K} 4 / 11$ (Chicoine et al. 1987) which corresponds to $\mathrm{H} 4 \mathrm{~K} 5 / 12 \mathrm{ac}$ in budding yeast and humans (see appendix 2-B for structure of acetylated lysine). In budding yeast and humans this conserved pattern of H4 diacetylation is carried out by Hat 1 complex (Parthun 2012).

Despite the identification of patterns of PTMs on newly synthesized H4, the Hat1 enzyme has not been identified or studied in $T$.thermophila. I began my analysis with BLASTP searches against $T$.thermophila MAC genome using as query yeast Hat1 and identified TTHERM_00046760 as the putative Hat1 enzyme $\left(\mathrm{e}^{-15}\right)$. Hat ${ }^{\mathrm{Tt}}$ shares a high degree of homology with other well characterized Hat1 enzymes from S. cerevisiae and $H$. sapiens (See below). Domain analysis using Pfam database indicates that similar to other family members, Hat1 ${ }^{\mathrm{Tt}}$ has N-terminal signature motif called Hat1_N (Pfam: PF10394) suggesting that it is a bonafide type-B family member. Crystal structures of S. cerevisiae and H. sapiens Hat1 enzymes have been resolved (PDB IDs: 1BOB and 2P0W, respectively)(Wu et al. 2012; Dutnall et al. 1998). In order to investigate the Hat $1^{\mathrm{Tt}}$ structural features, a three dimensional structure was predicted via homology modelling using PDB 1BOB. The predicted Hat $1^{\mathrm{Tt}}$ structure has several attractive features. For example, the core of the predicted structure is highly homologous to the reported human Hat1 structures (Figure 26A) suggesting that Hat $1^{\mathrm{Tt}}$ also belongs to the GNAT super-family. In fact, Hat ${ }^{\mathrm{Tt}}$ predicted structure can effectively be super-imposed with both $S$. cerevisiae and $H$. sapiens Hat1 structures (PDB IDs: 1BOB and 2P0W, respectively)(Wu et al. 2012; Dutnall et al. 1998) (Figure 26-B-C, respectively). A recent study showed that Hat1 interaction with Hat2 is 
stabilized by a few residues that form a long loop in the Hat1 structure (Li et al. 2014). These residues have been well conserved in Hat ${ }^{\mathrm{Tt}}$ (see Figure $26 \mathrm{D}$ ) and also form a loop on the predicted structure (see black highlight in Figure 26-B-C). Consistent with Hat ${ }^{\mathrm{Tt}}$ binding a Hat2 related protein. 


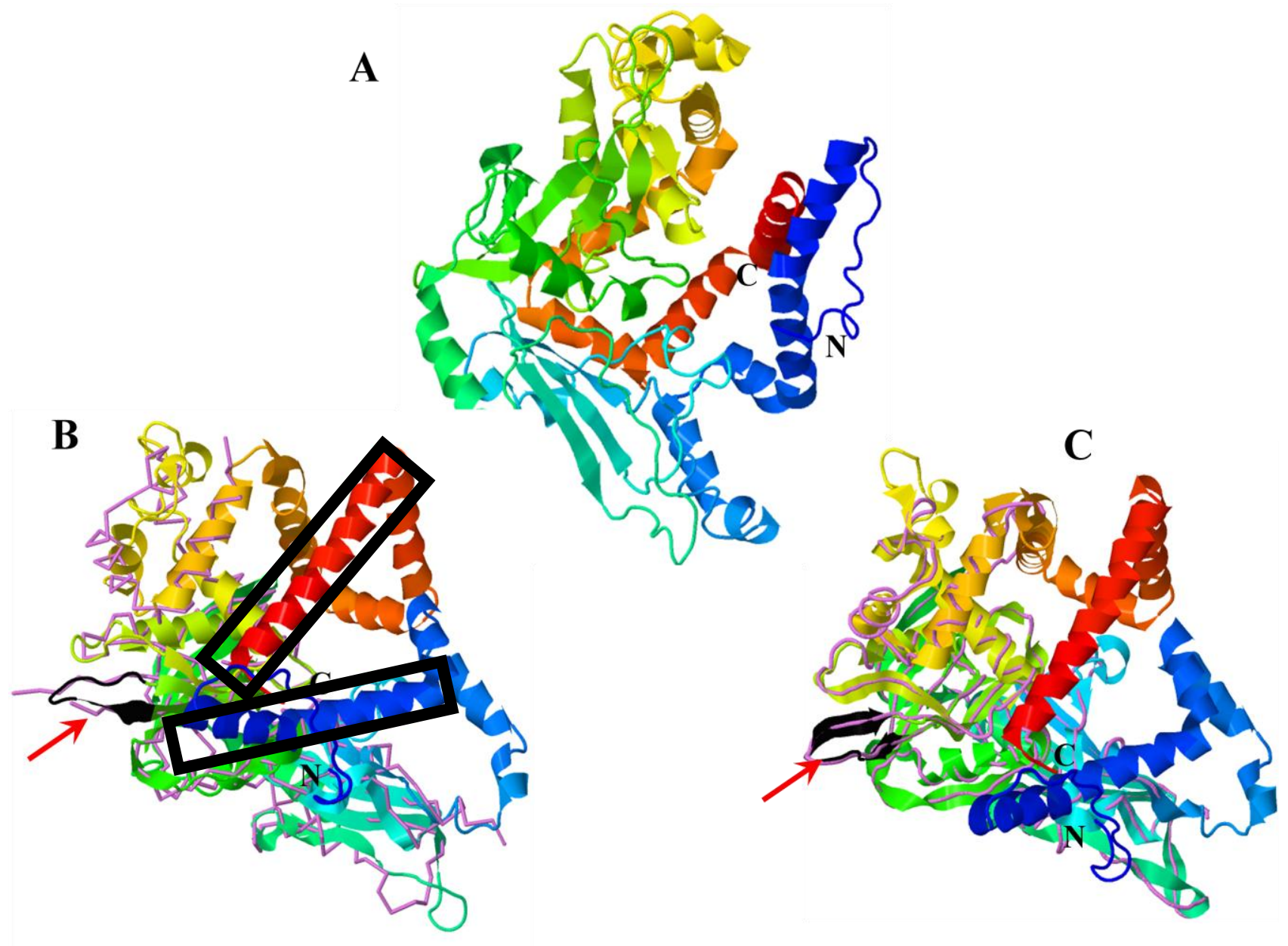

\section{D}

S. cerevisiae /1-17 KYWHYLGAKSFDED--IDK

T. thermophila /1-8 EYMFN - - . - . - .-INK

H. sapiens /1-8 NY Y VY - - - . - . - - -

C. elegans /1-8 KFY NY - - - - - - - IDR

Figure 26: Predicted tertiary structure of T. thermophila Hat1 protein. A- Overall three dimensional structure of the whole protein. The ribbon structure is coloured in rainbow scheme with dark blue representing $\mathrm{N}$ - terminal whereas red indicating the C-terminal. BHat ${ }^{\mathrm{Tt}}$ predicted structure superimposed with $S$. cerevisiae reported structure which is shown in backbone format and violet color. The red arrow points towards predicted loop (shown in black) for Hat 2 binding. The Boxes indicate the additional $\mathrm{N}$ - and $\mathrm{C}$ - termini that are present in $\mathrm{Hat}^{\mathrm{Tt}}$ and not in $S$. cerevisiae reported structure. C- Hat ${ }^{\mathrm{Tt}}$ predicted structure superimposed with $H$. sapiens reported structure (Violet backbone). D- Amino acid sequences of the loop region predicted to stabilize interaction with Hat2 (see text for details). 
Predicted functional annotation using COFACTOR server (Roy et al. 2012) of the predicted structure suggested that acetyl coenzyme A binds to the internal groove that lies toward the C-terminus of the protein (Figure 27) which is considerably consistent with the resolved Hat1 structure (Wu et al. 2012). Nevertheless, despite the overall high degree of homology Hat $1^{\mathrm{Tt}}$ has some divergent regions. For example, it has additional N- and Ctermini residues that are not found in S. cerevisiae and H. sapiens Hat1 proteins. These extra amino acids form several alpha helices that extend toward the front of the protein hindering the internal regions of the molecule (Figure 26-B). The function or significance of these divergent regions remains unknown.

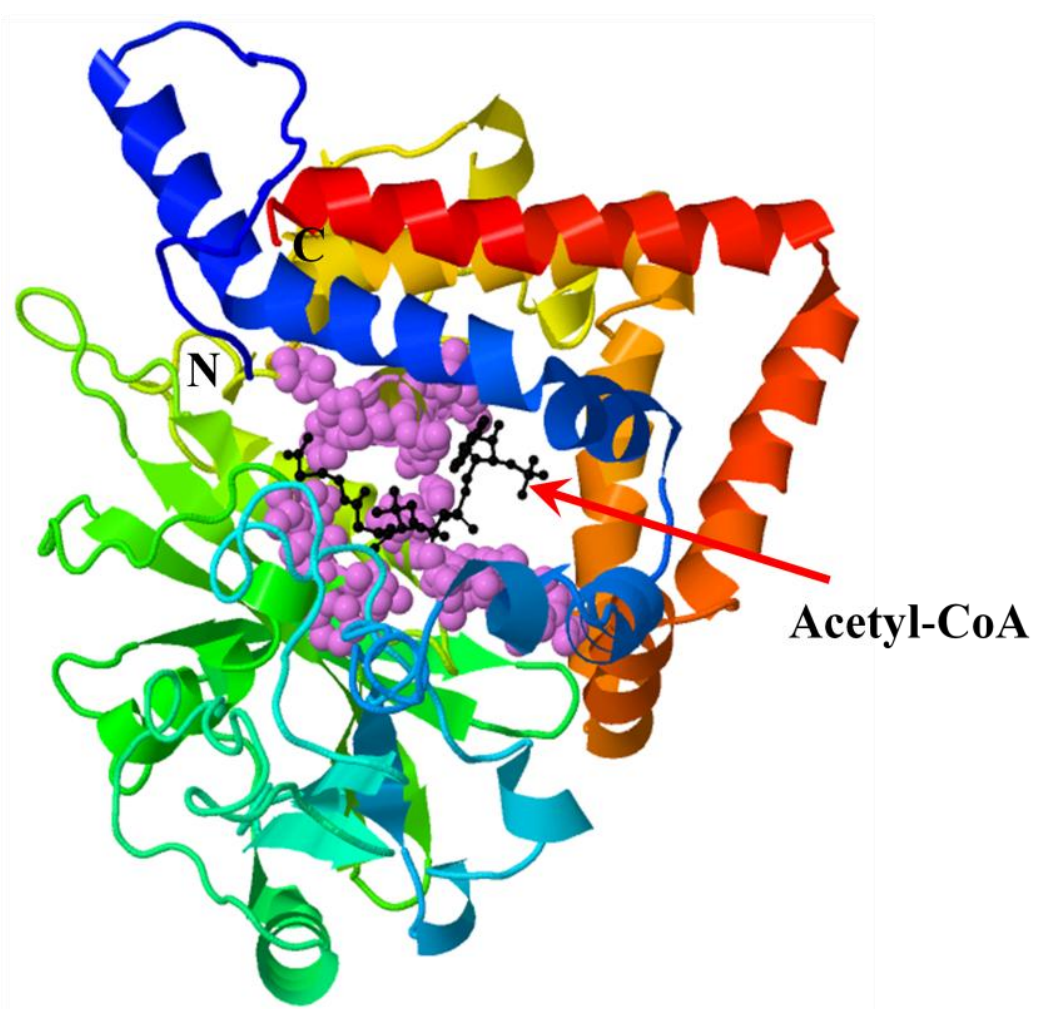

Figure 27: Acetyl-CoA bound to the predicted structure of Hat1 ${ }^{\mathrm{Tt}}$. The structure is shown in rainbow with blue and red representing $\mathrm{N}$ - and $\mathrm{C}$-termini, respectively. The Acety-CoA is shown in black stick representation whereas the binding sites on Hat $1^{\mathrm{Tt}}$ predicted structure are shown in CPK format. The binding sites include residues 234, 235, 286, 287, 288, 293, 294, 295, 296, 298, 299, 323, 324, 326, 327, 329, 330, 333. The functional annotation was carried out using CO-FACTOR server. 


\subsection{2- T. thermophila Hat1-complex is conserved}

To gain functional insights and reveal the proteomic composition of putative Hat1complex in T. thermophila, I generated stable cell lines expressing C-terminal FZZ epitope tagged Hat $1^{\mathrm{Tt}}$ from the native chromosomal loci. After cells finished phenotypic assortment, the expression of tagged proteins was examined by Western blots using WCEs prepared from either growing Hat $1^{\mathrm{Tt}}$-FZZ or wild type cells. Strong signals of approximately $78 \mathrm{kDa}$ were detected in the Hat $1^{\mathrm{Tt}}$-FZZ lanes and not in the un-tagged wild types when blots were probed with anti-FLAG antibody (Figure 28).

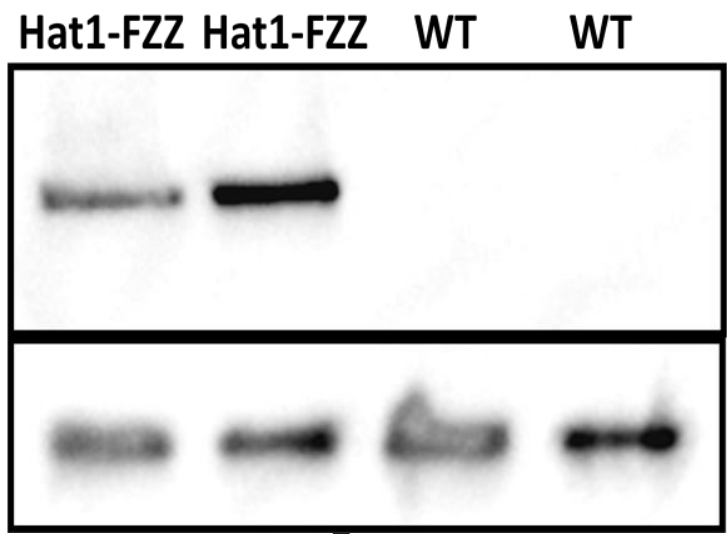

\section{Anti- FLAG}

Predicted size Hat1 $=60 \mathrm{kDa}$ FZZ predicted size $=18 \mathrm{kDa}$ Total fusion protein $=78 \mathrm{kDa}$

\section{Anti-Actin}

Figure 28: Western blot of Hat ${ }^{\text {Tt }}$-FZZ indicating the successful expression of the tagged protein in growing cells. Top panel was probed with anti-FLAG antibody which showed a strong signal in the lane corresponding to Hat ${ }^{\mathrm{Tt}}$-FZZ whereas no signal was detected in the wild type control. The band appeared within the reasonable range of the fusion protein predicted size $(\sim 78 \mathrm{kDa})$. The lower panel was probed with anti-actin antibody as a loading control.

Subsequently, pellets from the growing Hat $1^{\mathrm{Tt}}$-FZZ and wild type cells were prepared and subjected to the TAP procedure. Following the TAP, successfully recovery of the Hat $1^{\mathrm{Tt}}$ FZZ was assessed by Western blots probed with anti-FLAG antibody (Figure 29). 


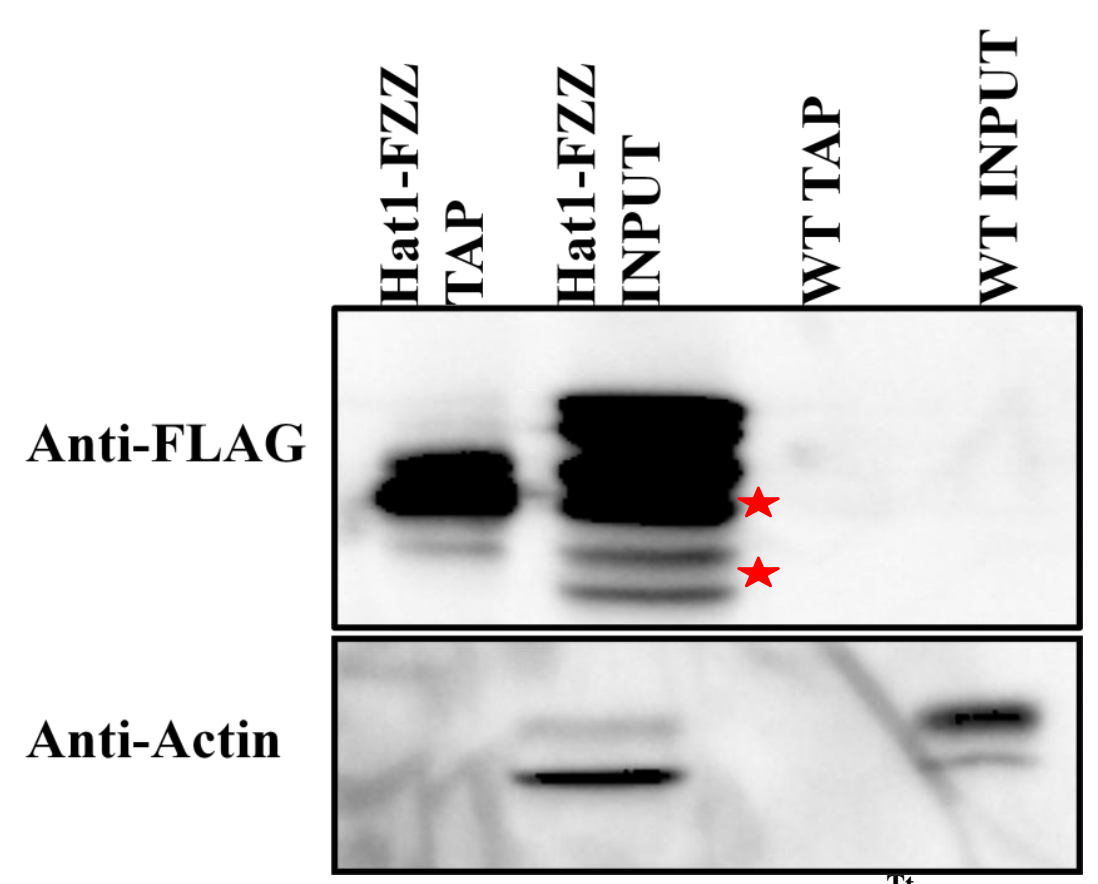

Figure 29: Successful recovery of purified Hat1 ${ }^{\text {Tt }}$-FZZ protein detected by Western blot. The blot was probed with anti-FLAG antibody to detect the signals in the Hat ${ }^{\mathrm{Tt}}$ input and TAP lanes. In contrast no signal was detected in the wild type lanes. Note, the size of Hat $1^{\mathrm{Tt}}$ in the TAP lane is smaller than the input lane indicating the removal of protein A part of the tag. The red stars indicate the proteolytic degradation. Bottom panel was probed with anti-actin as a loading control.

The purified material was then analysed by LC-MS/MS to detect any co-purifying proteins and SAINT analysis was used to differentiate between the Hat $1^{\mathrm{Tt}}$ specific and nonspecific interactions. As indicated in Table 4, Hat $1^{\mathrm{Tt}}$-FZZ co-purified reproducibly with a single protein which is encoded by TTHERM_00688660. Interestingly, the protein product of this gene was also identified as the putative $\mathrm{Cac} 3^{\mathrm{Tt}}$ subunit of the $\mathrm{CAF} 1^{\mathrm{Tt}}$ complex via purification and analysis of $\mathrm{Cac} 2^{\mathrm{Tt}}-\mathrm{FZZ}$ (see Table3). Domain analysis (see below) and BLAST searches indicated that TTHERM_00688660 (Hat2/Cac3 ${ }^{\mathrm{Tt}}$ hereafter) shares significant homology to both Hat2 as well as Cac3 proteins that are found in other organisms (BLAST scores $4 \mathrm{e}^{-145}, 5 \mathrm{e}^{-150}$ respectively). In S. cerevisiae and H. sapiens, Hat2 and Cac3 are encoded by two separate genes and share $90 \%$ homology to each other at protein level (Murzina et al. 2008). The observation that $\mathrm{Hat} 2 / \mathrm{Cac}^{\mathrm{Tt}}$ is encoded by a signal gene in $T$. thermophila suggests that this protein is component of at least two (and possibly more - see 
below) distinct protein complexes and might be capable of carrying out functions that are distributed between two separate genes in higher eukaryotes.

Table 4: AP-MS data filtered using SAINT analysis. The AvgP represents the overall probability of individual experimental replicates

\begin{tabular}{|c|c|c|c|c|c|}
\hline Bait & Prey & $\begin{array}{c}\text { Gene ID } \\
\text { Tetrahymena }\end{array}$ & $\begin{array}{r}\text { Spect } \\
\text { Samples }\end{array}$ & $\begin{array}{l}\text { unts } \\
\text { control }\end{array}$ & Avg P \\
\hline Hat $1^{\mathrm{Tt}}$ & Hat $^{\mathrm{Tt}}$ & TTHERM_00046760 & $5 / 106$ & $0 / 0 / 0$ & - \\
\hline Hat $1^{\mathrm{Tt}}$ & Hat $1 / \mathrm{Cac}^{\mathrm{Tt}}$ & TTHERM_00688660 & $5 / 335$ & $0 / 0 / 0$ & 1 \\
\hline
\end{tabular}

\subsection{3- T. thermophila Hat1 localizes to MAC only}

To investigate the sub-cellular localization patterns of putative Hat $1^{\mathrm{Tt}}$, I performed an indirect IF analysis using anti-FLAG primary antibody on vegetatively growing cells. As shown in figure 30 , Hat $1^{\mathrm{Tt}}-\mathrm{FZZ}$ localizes exclusively to the MAC during vegetative growth. The MAC localization is consistent with the several described functions of Hat1 including transcription related acetylation as well as chromatin assembly activities (Parthun 2012). In addition, weak signal was also observed in dividing MICs. Newly synthesized histones H4 are heavily acetylated during periods of active DNA replication and chromatin assembly by cytoplasmic Hat1-complex (Sobel et al. 1994; Parthun 2012). Interestingly, Hat1-FZZ cytoplasmic localization was clearly observed in dividing cells suggesting deposition related acetylation of newly synthesized histones $\mathrm{H} 3 / \mathrm{H} 4$. These observations are consistent with known functions of Hat1-complex in the deposition-related acetylation of newly synthesized histones. 


\section{$\underline{\text { Hat1-FZZ }}$}

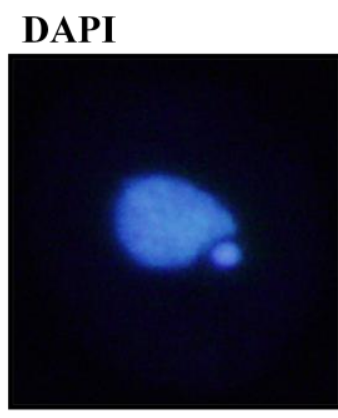

FITC
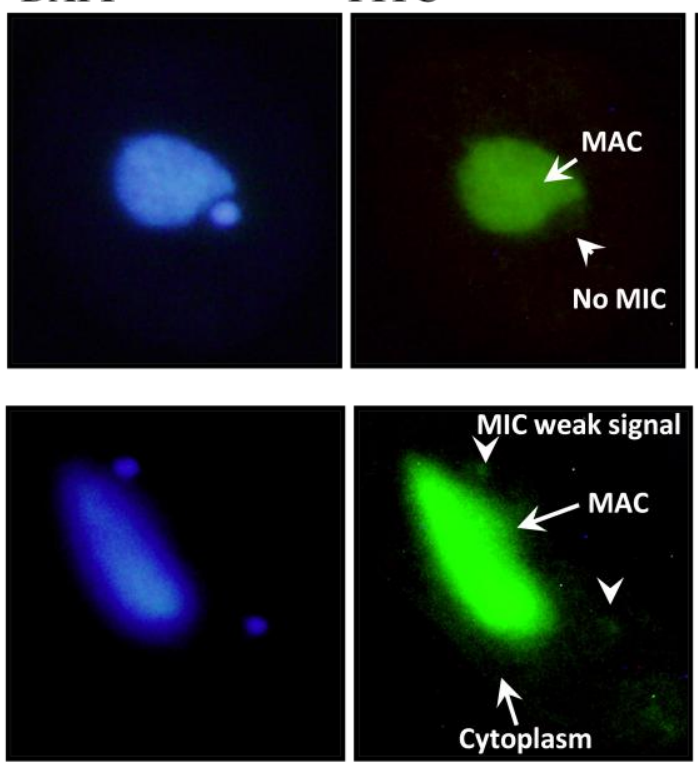

DAPI

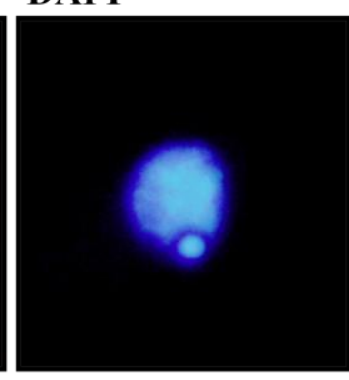

FITC
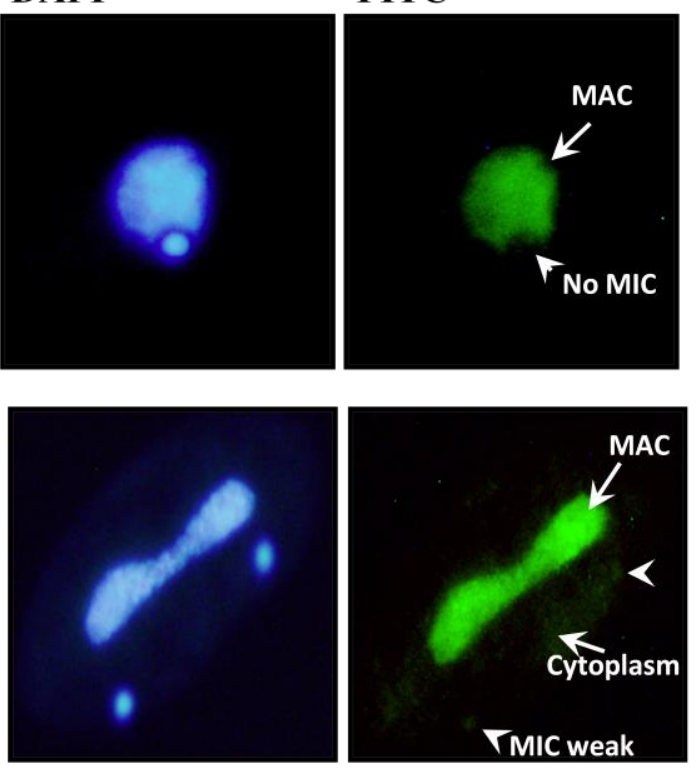

\section{WILD TYPE}

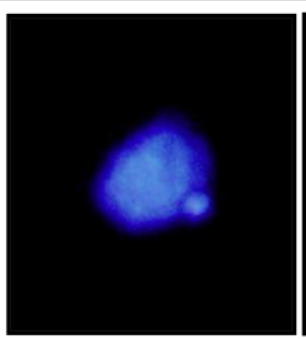

DAPI

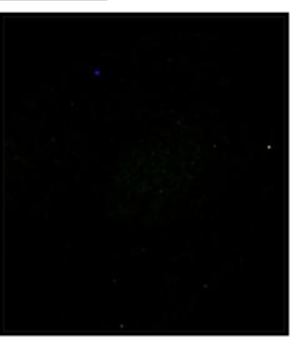

FITC

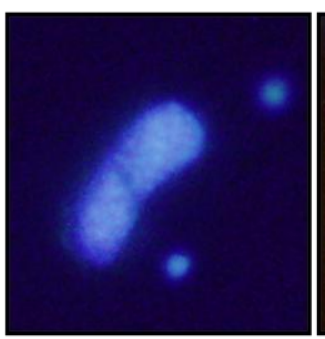

DAPI

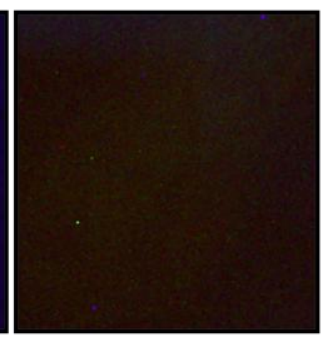

FITC

Figure 30: Indirect immunofluorescence of Hat1 ${ }^{\mathrm{Tt}}$-FZZ and untagged cells using antiFLAG antibody. Hat ${ }^{\mathrm{Tt}}$ predominantly localizes to MAC and faintly to MIC in dividing cells. Cells were stained with DNA-specific dye DAPI to capture the nuclei orientation. Arrow heads denote the position of MIC whereas open arrows indicate the MAC location. 


\section{5- Functional characterization of T. thermophila Hat2/Cac3}

Human RbAp46 and RbAp48 (also known as RBBP7 and RBBP4, respectively) are homologs of budding yeast Hat2 and Cac3 proteins, respectively. RbAp46 and RbAp48, encoded by two separate genes, are highly homologous proteins (90\% identical at the amino acid sequence level) and possess several WD40 repeats (Murzina et al. 2008). Together they are found as components of histone deacetylase (HDAC) complexes that function as transcriptional repressors of target genes including those repressed by the retinoblastoma tumor suppressor protein (Murzina et al. 2008). In addition, RbAp46 has a defined role as subunit of Hat1-complex whereas RbAp48 is exclusively found as subunit of CAF1 RD chromatin assembly complex (reviewed in Parthun 2012).

To begin to investigate the functions of single Hat $2 / \mathrm{Cac}^{\mathrm{Tt}}$ protein that is found as subunit of two separate complexes, i.e. Cac $3^{\mathrm{Tt}}$ subunit of $\mathrm{CAF} 1^{\mathrm{Tt}}$ and $\mathrm{Hat} 2^{\mathrm{Tt}}$ subunit of Hat $1^{\mathrm{Tt}}$ complex, I performed multiple sequence alignment analysis. Figure 31 shows that Hat2/Cac $3^{\text {Tt }}$ shares significant homology to both human RbAp46, RbAp48 and budding yeast Hat2, Cac3 proteins (>50\% similarity). This observation was further reinforced by the domain analysis (Figure 32) which showed that $\mathrm{Hat} 2 / \mathrm{Cac}^{\mathrm{Tt}}$ domain architecture is highly conserved. For example similar to human or budding yeast proteins, it has an $\mathrm{N}$-terminal CAF1-C H4-binding domain (H4-bd) (PFAM: PF12265) followed by six WD40 repeats. Crystal structures of human RbAp46/RbAp48 (PDB: 3CFS) (Murzina et al. 2008) as well as budding yeast Hat2 (PDB: 4PSX)( $\mathrm{Li}$ et al. 2014) proteins have been resolved. These structures have 7-bladed $\beta$-propeller fold typical of other WD40 repeats proteins such as WDR5. As expected from high degree of amino acid conservation Hat $2 / \mathrm{Cac} 3^{\mathrm{Tt}}$ predicted structure also adopts 7-bladed $\beta$-propeller fold which is nearly identical to human RbAp46/RbAp48 (PDB: 3CFS) (Figure 33-A, B). RbAp46/RbAp48 structure has small 
negatively charged loop (residues Ser-347 to Glu-364) which ends with two Proline (Pro362/Pro-363) residues and hence is called as PP-loop. The PP-loop has been shown to stabilize interaction with $\mathrm{H} 4$ as it forms a distinct pocket with $\mathrm{N}$-terminal alpha helix (See figure 33-B) (Murzina et al. 2008). Interestingly, Hat2/Cac3 ${ }^{\mathrm{Tt}}$ also forms this loop suggesting the potential pocket of $\mathrm{H} 4$ binding on the predicted structure. In fact the residues of this loop are highly conserved. The PP-loop residues Glu-356, Asp-357, and Asp-360 in RbAp46 have been shown to be important for H4 binding (Murzina et al. 2008) and they appear unchanged in the Hat $2 / \mathrm{Cac}^{\mathrm{Tt}}$ as well (Figure $33 \mathrm{C}$ ).

In addition, Hat $2 / \mathrm{Cac} 3^{\mathrm{Tt}}$ predicted structure can be superimposed with RbAp46/RbAp48 (Figure 33-D).This superimposition also revealed a region encompasses Leu90-Gly115 that is divergent in Hat2/Cac $3^{\mathrm{Tt}}$. MSA analysis also indicates that this region is relatively less conserved in Hat $2 / \mathrm{Cac}^{\mathrm{Tt}}$ (Figure 31). It adopts a loop surrounding the blades 6 and 7 on the $\beta$-propeller. This region may participate in lineage specific functions of Hat $2 / \mathrm{Cac}^{\mathrm{Tt}}$. 


\begin{tabular}{|c|c|c|}
\hline RBBP4 & 1 & - MA D K E A A F D DAVEERV - I NEEYK I WKKN TPF L Y D LVMTHALEWPS 44 \\
\hline Cac3 & 1 & IN Q C A KD I T HEAS S I P I D LQERY S HWKKN TKL L Y D Y L N TNSTKWPS \\
\hline $\mathrm{Hat} 2 / \mathrm{Cac} 3^{\mathrm{Tt}}$ & 1 & IN Q QMAE E F N DGYENQA - I NDEYK I WKKNAPF L Y D I A I THELEWPS \\
\hline RBBP7 & 1 & - MA S KEMF E DTVEERV - I NEEYK I WKKNTPF L Y DL VMTHALQWPS \\
\hline HAT2 & 1 & - - - - - MENQEKPLS VDEEY DLWKS NVPLMY DFV S E TRLTWPS \\
\hline RBBP4 & 45 & L T A QWL P D V T R PEGKDFS I HRLVLGTHT S D - - E QNHL V IASVQLP \\
\hline Cac3 & 7 & L T C Q F F P D L D T T S DE - - - - HR I L L S S FT S SQKP E DET I Y I SK I \\
\hline Hat $2 / \mathrm{Cac}^{\mathrm{Tt}}$ & 6 & L S VQWL P T KD I PQESDYA I HKL I LGTHT S GQ - - D KDY L L I AKVR LP \\
\hline RBBP7 & 4 & L T V QWL P E V T KPEGKDYA LHWL V LGTHT S D - - E QNH L VVARVH \\
\hline HAT2 & 38 & L T V QWL P T P V QELDGGF I KQEL I I GTHT S GE - - E ENY L KFAE I \\
\hline RBBP4 & 88 & F D A SHYDS EKGEF - - - G - - GF GS V SGK I E I E I K I NHEGE VN 12 \\
\hline Cac3 & 89 & GH I KW S S L N F DMDEMEF KP EN S TR F PS KHLVND I S I - F FPNGE CN 133 \\
\hline Hat $2 / \mathrm{Cac} 3^{\mathrm{Tt}}$ & 90 & LE E T A T D I S E YQNQAKEV - - - GQTGL SAGENR I E I E T K I LHDGE IN 132 \\
\hline RBBP7 & 87 & NDDA Q F D A S H CDS DKGEF - - - G - - GF GS V TGK I E C E I K I NHEGE VN 127 \\
\hline HAT2 & 82 & NE - - DPQEEAGEE - - YQSSI \\
\hline RBBP4 & 29 & I A TKTPS S DVLV FDY \\
\hline Cac3 & & R A R Y L P Q N P D I I A GAS SDGA I Y I FDR TKHGSTR I RQS K I SHPFE TK 179 \\
\hline Hat $2 / \mathrm{Cac}^{\mathrm{Tt}}$ & 33 & R A R YMPQKYN V I A TKVTNGE I HV F DY TQHPTTP Q - NDQ VRPQL - - R 175 \\
\hline RBBP7 & 128 & IPQN PH I I A TKT P S S DVLV \\
\hline HAT2 & 123 & R YMPQDPN I VA T - - I NGQGTV FLY SR - - - - - SEG LQSTL - - K 15 \\
\hline RBBP4 & 173 & LSWNPNL S GHL L S ASDDHT \\
\hline Cac3 & & S HGV I QD VEAMDT S S AD I NEATS \\
\hline Hat $2 / \mathrm{Cac}^{3 t}$ & 176 & LVGHS _ . . . . . . . . . . AE GYG I SWNPKK Q GY I V S GGYDKK I 205 \\
\hline RBBP7 & 172 & LRGHQ - - - - - - . - - - KE GYGLSWNSNL S GHL L S ASDDHTV 201 \\
\hline HAT2 & 158 & F - - HK - - - . - . - . - DNGYALS F S TLV K GRL L S GSDDHTV 185 \\
\hline RBBP4 & & \\
\hline $\mathrm{Cac} 3$ & 226 & VD I K Q Y S HENP I \\
\hline Hat $2 / \mathrm{Cac}^{\mathrm{Tt}}$ & 206 & VNVEA A S Q LNS S I S - P LHDI E FHKSC \\
\hline RBBP7 & 202 & VD I N A G P K EGK I VD - A KA I F T GHS AV \\
\hline HAT2 & 186 & VR - TWNDL - - HS \\
\hline RBBP4 & 248 & NNTS - - KPSH \\
\hline Cac3 & 272 & E GNA V S L L D L RTKKE - - - KLQS NRE \\
\hline Hat $2 / \mathrm{Cac} 3^{\mathrm{Tt}}$ & 251 & D D R T V A I WDMRQK S NAGL I NPTHC TQAHT GD I Y C L DF N P FNEY I \\
\hline RBBP7 & 247 & M I WD T R SNTTS - - - KP SHLVD \\
\hline HAT2 & 229 & DV RANNTT - - I DTVK C PQPF - - - - N T LAF S HHS SNL LA 268 \\
\hline RBBP4 & 291 & T G S A D K T VA L WDL RNLK \\
\hline Cac3 & 314 & GT S VST L E WS P N F D \\
\hline $\mathrm{Iat} 2 / \mathrm{Cac} 3^{\mathrm{Tt}}$ & 297 & TDQVLRC EWS P F NVC \\
\hline BBP7/1 & 290 & $\mathrm{~T} \mathrm{G}$ \\
\hline AT2/1-401 & 269 & 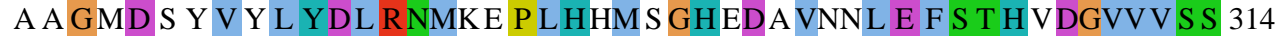 \\
\hline BB & & $\mathrm{G}$ \\
\hline Cac3/1-4 & 360 & - CEET I F T H \\
\hline Hat $2 / \mathrm{Cac}^{\mathrm{Tt}}$ & 343 & I S KCGQEMKNEDLVD \\
\hline RBBP7 & 336 & G T D R R L N V - V \\
\hline HAT2 & 315 & G S DNR L MM - WDLKQ I GAE QT PDDAEDGV P EL I MV HAG HR S \\
\hline RBBP4 & & D-FEUQU \\
\hline Cac3 & 392 & KPAGNL_ - . . - - - VC \\
\hline $\mathrm{Hat} 2 / \mathrm{Cac}^{\mathrm{Tt}}$ & 388 & NQKE NL I L A S VE ENN I L QVWQMARN I - YDDTDD EVMKD - . . . - \\
\hline RBBP7 & 381 & MAEN I - YNDEE S DVT T S ELEGQGS 42 \\
\hline HAT2 & & \\
\hline
\end{tabular}

Figure 31: Multiple sequence alignments of human RBBP4/7, budding yeast Hat2 and T. thermphila Hat1/Cac3 ${ }^{\mathrm{Tt}}$. The color coding is clustalx (See appendix 2). 


\section{H. Sapiens RBBP4}

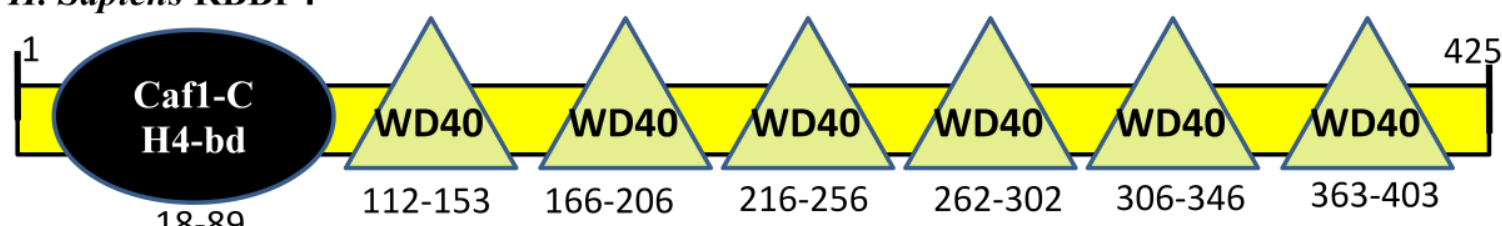

S. cerevisiae Cac3

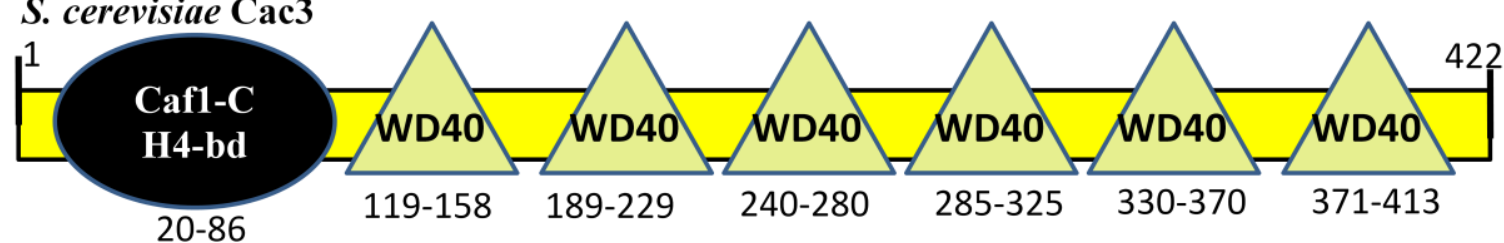

T. thermophila Hat2/Cac3

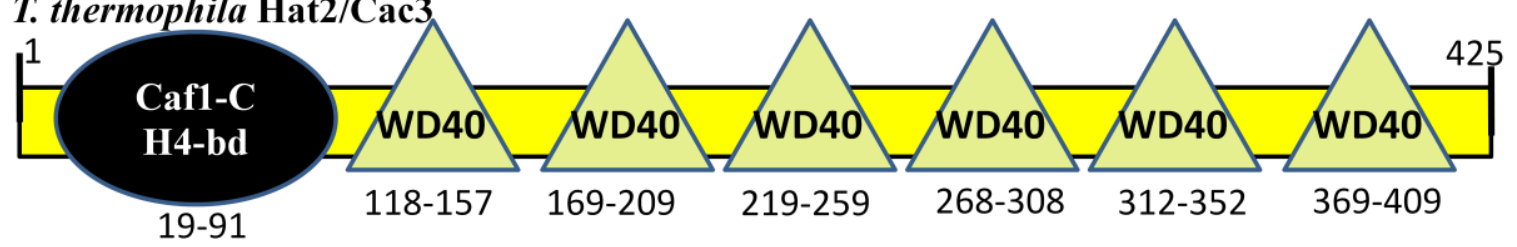

H. Sapiens RBBP7

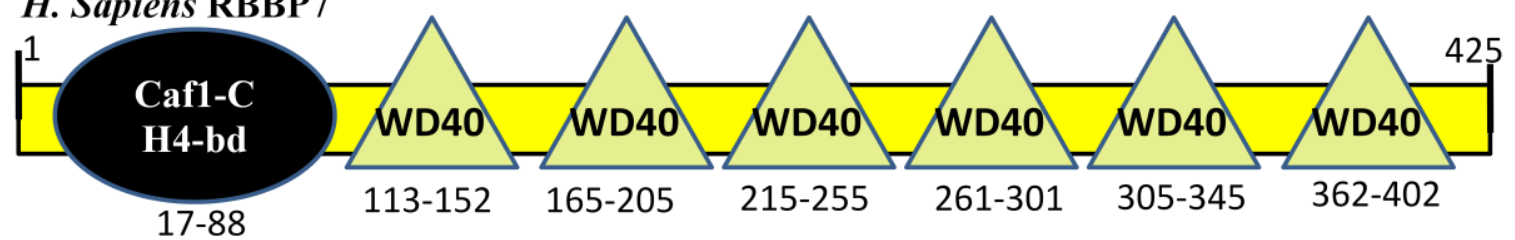

S. cerevisiae Hat2

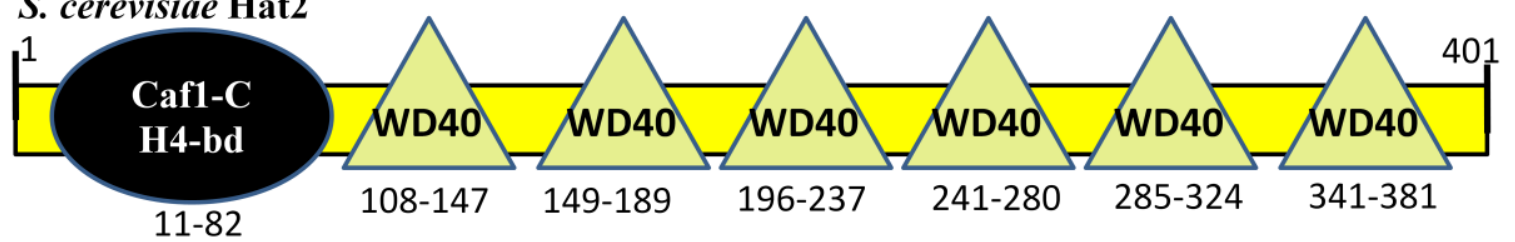

Figure 32: Structural comparison of various Hat2/Cac3Tt homologs. The figure is based on SMART and PFAM domain analysis. The number and positions of the amino acid residues comprising each domain are also indicated. 


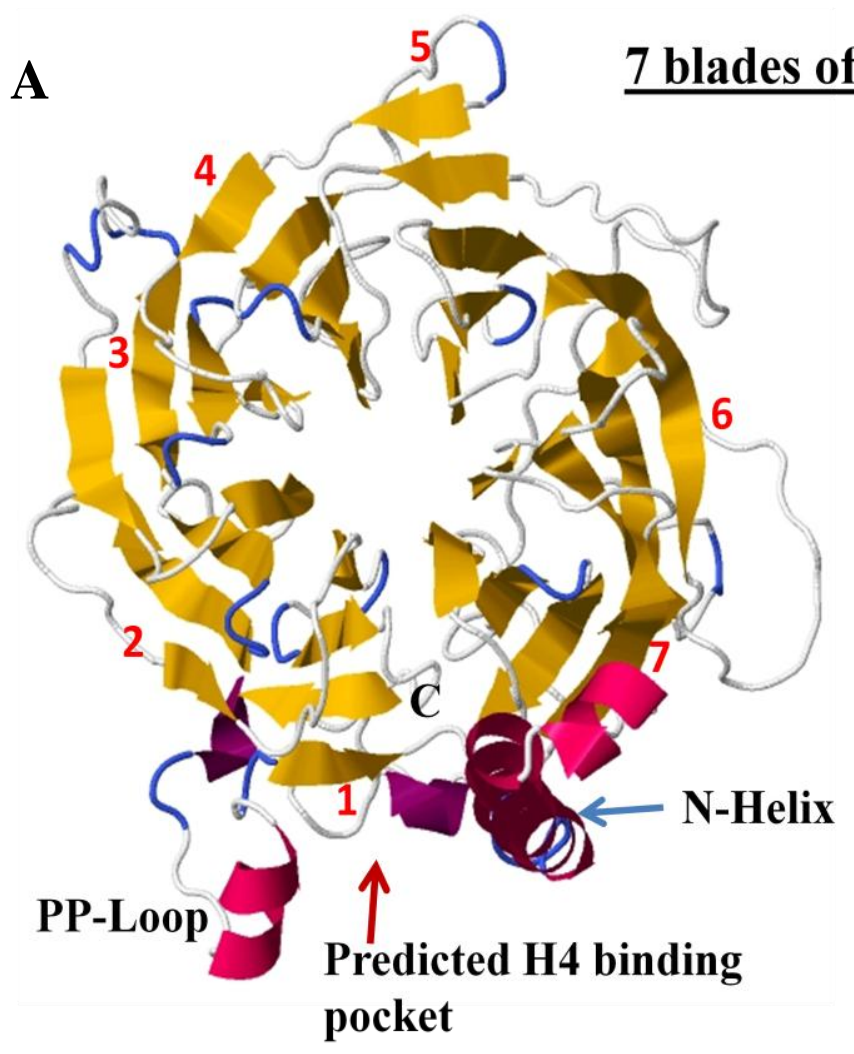

Hat $2 / \mathrm{Cac}^{\mathrm{Tt}}$ predicted structure

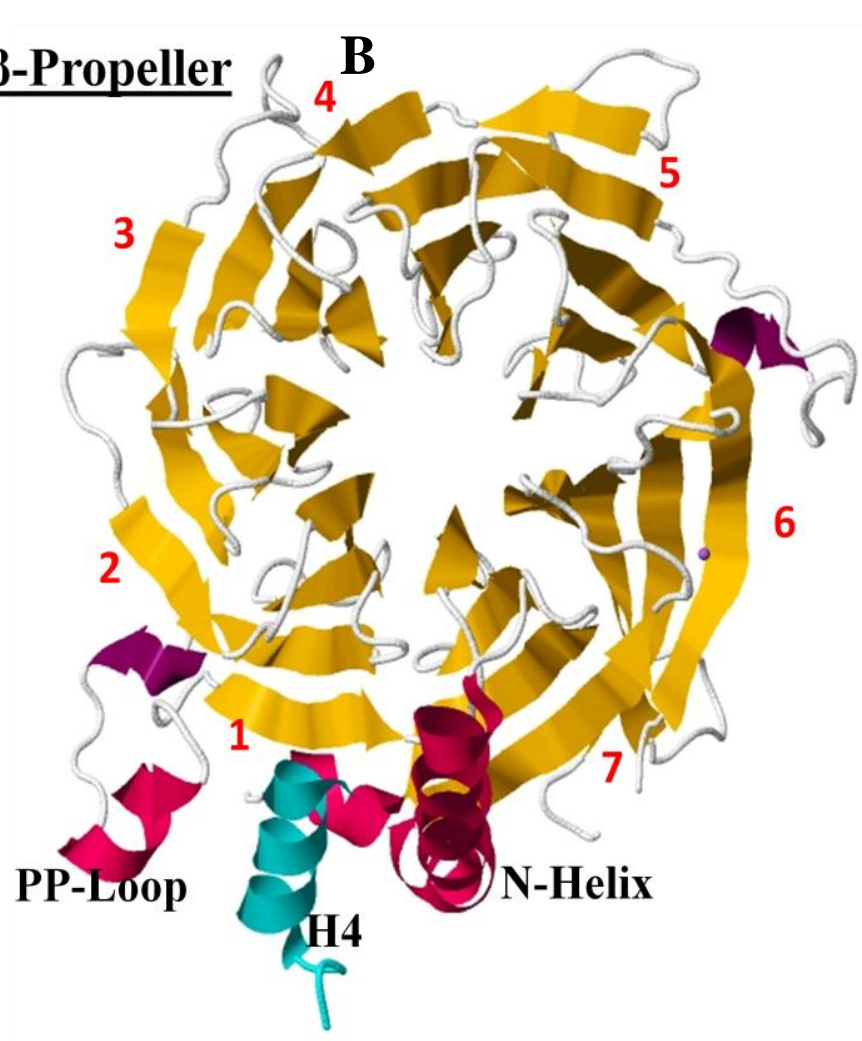

Resolved Structure of RbAp46/RbAp48 with histone $\mathrm{H4}$

C

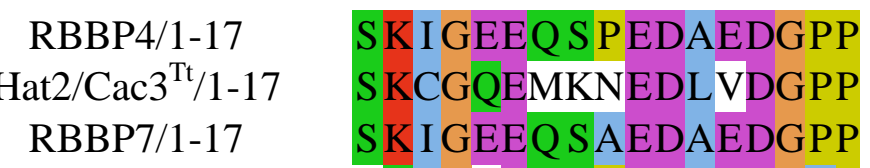

S. cerevisiae Hat2/1-17 KQ IGAEQTPDDAEDGVP 


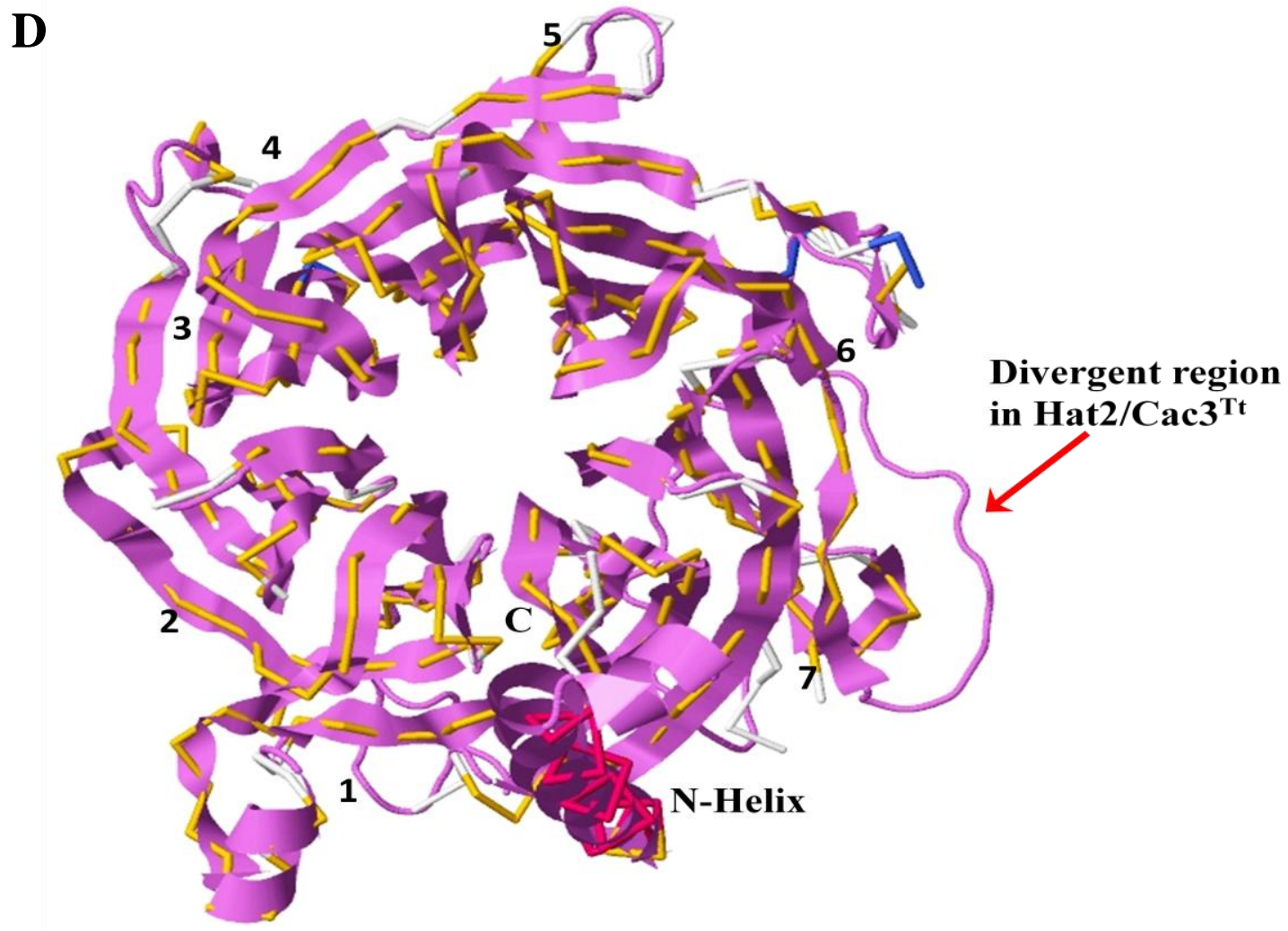

Figure 33: Predicted tertiary structure of $T$. thermophila $\mathrm{Hat}^{2} / \mathrm{Cac}^{\mathrm{Tt}}$ protein in comparison to the reported RbAp46/ RbAp48 structure. A- Predicted structure of Hat $2 / \mathrm{Cac}^{\mathrm{Tt}}$. Seven blades are consecutively numbered from 1-7 in accordance with those of human RbAp46/ RbAp48 structure reported by (Dutnall et al. 1998). Different region of the predicted structure are labelled in comparison with human RbAp46/ RbAp48 structure BReported structure of RbAp46/ RbAp48 structure (Dutnall et al. 1998) C- PP-loop sequence is shown for different lineages in clustalx color scheme. D- Superimposition of Hat $2 / \mathrm{Cac} 3^{\mathrm{Tt}}$ predicted structure (violet ribbons) and $\mathrm{RbAp} 46 / \mathrm{RbAp} 48$ structure (gold backbone). Arrow indicates divergent region on $\mathrm{Hat} 2 / \mathrm{Cac} 3^{\mathrm{Tt}}$ predicted structure 


\subsection{2- Hat2/Cac3 ${ }^{\mathrm{Tt}}$ is a component of multiple complexes}

To further functionally characterize Hat2/Cac3 and reciprocally verify observed physical interactions with putative $\mathrm{Caf} 1^{\mathrm{Tt}}$ complex and Hat $1^{\mathrm{Tt}}$, I engineered stable $T$. thermophila cell lines expressing Hat2/Cac ${ }^{\mathrm{Tt}}$ carrying $\mathrm{C}$-terminal FZZ epitope tag from its native chromosomal locus. The successful expression of the tagged protein was detected by Western blots using WCEs prepared from Hat2/Cac $3^{\mathrm{Tt}}-\mathrm{FZZ}$ and un-tagged wild type cell lines. As shown in figure 34, when probed with anti-FLAG antibody, strong signal was detected in the Hat2/Cac3 ${ }^{\mathrm{Tt}}$-FZZ lanes and not in that of un-tagged wild type.

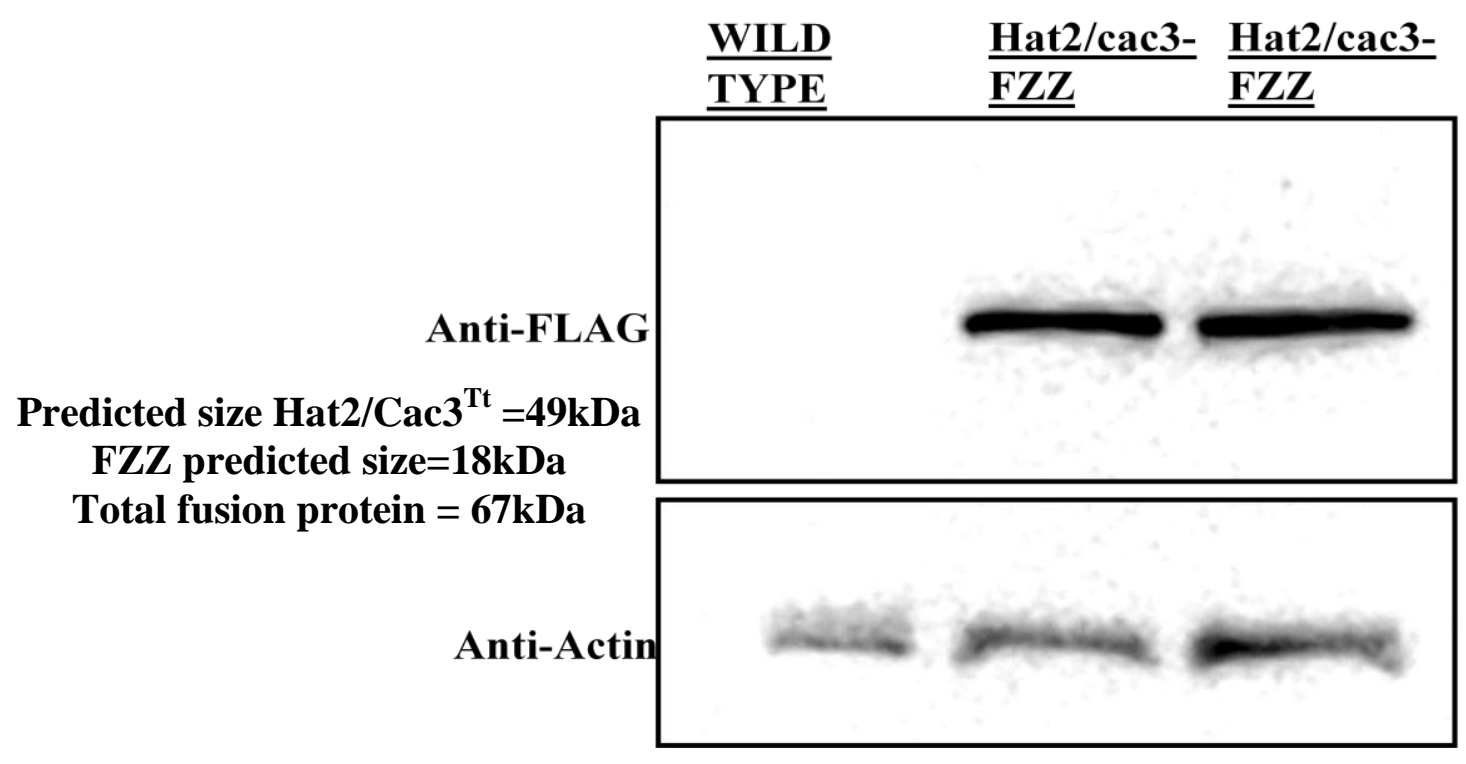

Figure 34: Western blot of Hat $2 / \mathrm{Cac}^{\mathrm{Tt}}$-FZZ indicating the successful expression of the tagged protein in growing cells. Top panel was probed with anti-FLAG antibody which showed a strong signal in the lane corresponding to Hat $2 / \mathrm{Cac}^{2 \mathrm{Tt}}-\mathrm{FZZ}$ whereas no signal was detected in the wild type control. The band appeared within the reasonable range of the fusion protein predicted size $(\sim 70 \mathrm{kDa})$. The lower panel was probed with anti-actin antibody as a loading control.

After detecting Hat $2 / \mathrm{Cac}^{\mathrm{Tt}}-\mathrm{FZZ}$ successful expression, I prepared cell pellets from vegetatively growing cells to perform affinity purification. The successful recovery of the purified Hat2/Cac ${ }^{\mathrm{Tt}}$-FZZ was assessed by Western blots probed with anti-FLAG antibody. As indicated in Figure 35, strong signal were detected in the Hat2/Cac $3^{\mathrm{Tt}}-\mathrm{FZZ}$ input and 
affinity purified lanes whereas no signal was observed in the wild type lanes. Subsequently, the affinity purified material was analyzed by LC MS/MS.

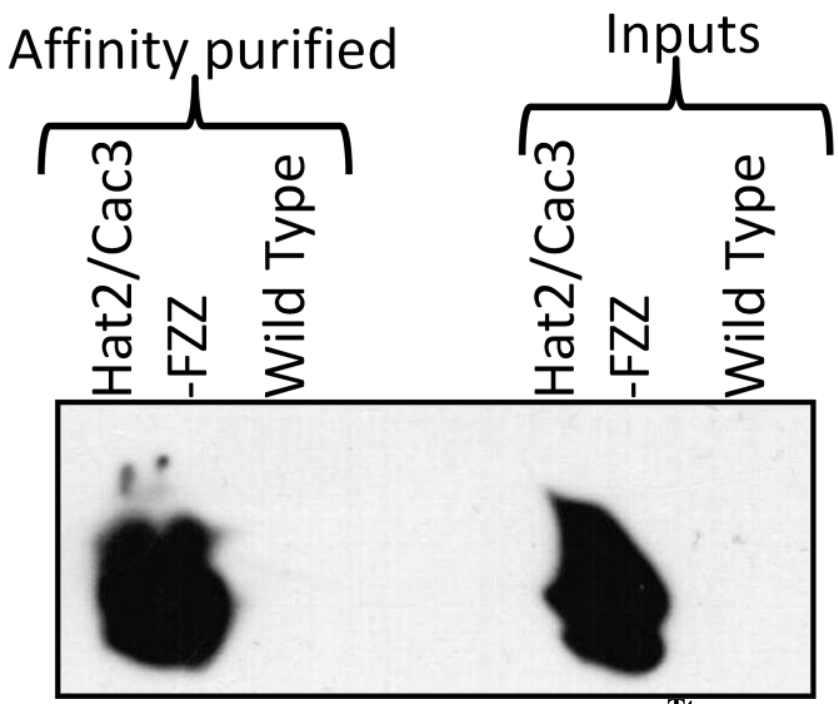

Figure 35: Successful recovery of purified Hat2/Cac3 ${ }^{\mathrm{Tt}}-\mathrm{FZZ}$ protein detected by Western blot. The blot was probed with anti-FLAG antibody to detect the signals in the Hat $2 / \mathrm{Cac}^{\mathrm{Tt}}$-FZZ input and TAP lanes. In contrast no signal was detected in the wild type lanes. Bottom panel was probed with anti-actin as a loading control.

The MS data presented in Table 5 summarises the Hat2/Cac $3^{\mathrm{Tt}}-\mathrm{FZZ}$ co-purifying proteins as assessed by comparison against three of wild type control purifications. Table 5 indicates that $\mathrm{Hat} 2 / \mathrm{Cac}^{\mathrm{Tt}}-\mathrm{FZZ}$ co-purifies with putative $\mathrm{Cac1}^{\mathrm{Tt}}$ (TTHERM_00309890) subunit of $\mathrm{CAF}^{\mathrm{Tt}}$ and Hat $1^{\mathrm{Tt}}$ (TTHERM_00046760) complexes. As noted earlier, Cac1 ${ }^{\mathrm{Tt}}$ also co-purifies with $\mathrm{Cac} 2^{\mathrm{Tt}}$ (see Table 3 ) as subunit of putative $\mathrm{CAF} 1^{\mathrm{Tt}}$ complex. Taken together, these data suggested that $\mathrm{Hat} 2 / \mathrm{Cac}^{\mathrm{Tt}}$ exists as subunit of two independent yet functionally linked protein complexes that are central to chromatin assembly pathways, i.e. Hat $1^{\mathrm{Tt}} / \mathrm{Hat} 2^{\mathrm{Tt}}$ and $\mathrm{CAF} 1^{\mathrm{Tt}}$. The MS data also reveals that Hat2/Cac3 ${ }^{\mathrm{Tt}}-\mathrm{FZZ}$ co-purifies with several additional proteins.

The first Hat2/Cac3 co-purifying novel protein is encoded by TTHERM_00227070 and neither has any recognisable domains other than three coiled-coiled regions as predicted 
by SMART, nor does it share homology to any proteins when searched against nonredundant database using BLASTP. Nevertheless, eggNOG orthology database indicated that TTHERM_00227070 forms a protein family of three members (euNOG 34075). Remaining two family members include an uncharacterized protein (accession\# Q8IKH4) from a related apicomplexan Plasmodium falciparum and a protein of unknown function (accession\# Q5C3T9) from a metazoan parasitic lineage Schistosoma japonicum. I have renamed TTHERM_00227070 as CPP1 protein based on available TIGR gene annotation TIGR00225 which indicated that it has a $\boldsymbol{C}$-terminal processing $\boldsymbol{p}$ eptidase domain.

Interestingly MS data also indicated that Hat $2 / \mathrm{Cac}^{\mathrm{Tt}}$ interacts with several additional proteins, including TTHERM_00450950 which shares homology to transcriptional repressor Sin3 protein as assessed by BLASTP searches. Sin3 forms a multi-protein histone deacetylase (HDAC) SIN3 complex which has HDAC1/2 as its catalytic subunits and functions in transcriptional repression (reviewed in Grzenda et al. 2009). The presence of Hat $2 / \mathrm{Cac}^{\mathrm{Tt}}$ as subunit of putative SIN $3^{\mathrm{Tt}}$ complex is consistent with previous findings which have shown RBBP4/7 as component of SIN3 complex in other organisms including humans (reviewed in Grzenda et al. 2009). In addition BLAST searches identified TTHERM_00766460 as a putative homolog of human tumor suppressor LIN9 protein. Similarly, TTHERM_00992830 shares homology with Rxt3 which is a component of RPD3L HDAC complex which also includes $\operatorname{Sin} 3$ as its functional partner and functions in transcription regulation (Ruiz-Roig et al. 2010). T. thermophila genome encodes 19 putative HDACs (THD1-18) as assessed by BLAST searches as well as available genome annotations (www.ciliate.org). Hat2/Cac3 $3^{\mathrm{Tt}}$ interactions with $\mathrm{Sin} 3$ and Rxt3 suggest that it is a component of HDAC complex however no putative THD was co-purified. 
Two PHD zinc finger containing proteins TTHERM_00666310 and TTHERM_00420920 also co-purified with Hat2/Cac3 ${ }^{\mathrm{Tt}}$. PHD finger proteins have been implicated in a large number of cellular processes including DNA/RNA binding (Musselman and Kutateladze 2009). In addition to zinc finger proteins, putative homolog of TESMIN/TSO1 protein TTHERM_00766460 also co-purified. TESMIN/TSO1 protein has been implicated in developmental regulation and control of cell division (Sugihara et al. 1999) (See discussion).

Taken together these data suggest that T. thermophila Hat $2 / \mathrm{Cac} 3^{\mathrm{Tt}}$ forms several stable protein complexes and is functionally diverse protein with roles in chromatin assembly, histone PTMs and transcription regulation. In order to perform SAINT analysis, we are currently attempting additional purifications of Hat $2 / \mathrm{Cac}^{\mathrm{Tt}}-\mathrm{FZZ}$ from whole cell extracts made from this cell line. 
Table 5: AP-MS data using Hat2/Cac $3^{T t}-F Z Z$ as bait. Data was compared against three mock purifications

\begin{tabular}{|c|c|c|c|c|}
\hline \multirow[t]{2}{*}{ Bait } & \multirow[t]{2}{*}{ Prey } & \multirow[t]{2}{*}{ Gene ID } & \multicolumn{2}{|c|}{ Spect. Counts } \\
\hline & & & Sample & Controls \\
\hline Hat2/Cac3 ${ }^{\mathrm{Tt}}$ & Hat $2 / \mathrm{Cac}^{\mathrm{Tt}}$ & TTHERM_00688660 & 158 & $0 / 0 / 0$ \\
\hline Hat $2 / \mathrm{Cac}^{\mathrm{Tt}}$ & SIN3 ${ }^{\mathrm{Tt}}$ & TTHERM_00450950 & 132 & $0 / 0 / 0$ \\
\hline Hat2/Cac $3^{\mathrm{Tt}}$ & Novel/CPP1 & TTHERM_00227070 & 100 & $0 / 0 / 0$ \\
\hline Hat $2 / \mathrm{Cac} 3^{\mathrm{Tt}}$ & $\begin{array}{c}\text { Tesmin/TSO1- } \\
\text { like }^{\mathrm{Tt}}\end{array}$ & TTHERM_00766460 & 20 & $0 / 0 / 0$ \\
\hline Hat $2 / \mathrm{Cac}^{\mathrm{Tt}}$ & $\begin{array}{l}\text { PHD-finger } \\
\text { protein }^{\mathrm{Tt}}\end{array}$ & TTHERM_00420920 & 19 & $0 / 0 / 0$ \\
\hline Hat $2 / \mathrm{Cac}^{\mathrm{Tt}}$ & LIN9 $^{\mathrm{Tt}}$ & TTHERM_01000180 & 16 & $0 / 0 / 0$ \\
\hline Hat2/Cac $3^{\mathrm{Tt}}$ & $\mathrm{RXT3}^{\mathrm{Tt}}$ & TTHERM_00992830 & 13 & $0 / 0 / 0$ \\
\hline Hat $2 / \mathrm{Cac}^{\mathrm{Tt}}$ & Hat $1^{\mathrm{Tt}}$ & TTHERM_00046760 & 9 & $0 / 0 / 0$ \\
\hline Hat2/Cac $3^{\mathrm{Tt}}$ & PHD-finger $^{\mathrm{Tt}}$ & TTHERM_00666310 & 5 & $0 / 0 / 0$ \\
\hline Hat $2 / \mathrm{Cac}^{\mathrm{Tt}}$ & ${\text { Ubiquitin } \mathrm{E} 2^{\mathrm{Tt}}}^{\mathrm{Tt}}$ & TTHERM_01082930 & 5 & $0 / 0 / 0$ \\
\hline Hat $2 / \mathrm{Cac}^{\mathrm{Tt}}$ & $\begin{array}{l}\text { Dead Box } \\
\text { heliacse }^{\mathrm{Tt}}\end{array}$ & TTHERM_00189460 & 3 & - \\
\hline Hat $2 / \mathrm{Cac}^{\mathrm{Tt}}$ & $\begin{array}{l}\text { Ser/Thre } \\
\text { Kinase }\end{array}$ & TTHERM_00189460 & 3 & \\
\hline Hat $2 / \mathrm{Cac}^{\mathrm{Tt}}$ & $\mathrm{Cac1}^{\mathrm{Tt}}$ & TTHERM_00309890 & 3 & $0 / 0 / 0$ \\
\hline
\end{tabular}




\subsection{3- Hat2/Cac3 ${ }^{\mathrm{Tt}}$ localization in MIC is cell cycle dependent}

To gain such functional insights an indirect IF analysis was performed using vegetative Hat2/Cac ${ }^{\mathrm{Tt}}$-FZZ cell lines (Figure $36 \mathrm{~A}-\mathrm{E}$ ). The analysis indicates that during vegetative growth Hat2/Cac $3^{\mathrm{Tt}}$ is mainly found in the MAC and not in the MIC (Figure 36A, B). As noted above, Hat $2 / \mathrm{Cac} 3^{\mathrm{Tt}}$ exists as subunit of at least three and probably more separate protein complexes including Hat $1^{\mathrm{Tt}}$ and $\mathrm{CAF}^{\mathrm{Tt}}$ and $\mathrm{SIN} 3^{\mathrm{Tt}}$. The observed MAC localization pattern of Hat $2 / \mathrm{Cac}^{\mathrm{Tt}}$ is consistent with that of Hat $1^{\mathrm{Tt}}$ but not with that of $\mathrm{Cac}^{\mathrm{Tt}}$ as described above (compare Figures 24, 30 and 36). Interestingly, although I did not observe Hat $2 / \mathrm{Cac}^{\mathrm{Tt}}$ in interphase T.thermophila during IF analysis (Fig 36A, B), Hat2/Cac $3^{\text {Tt }}$-FZZ localization to the MIC was observed when it starts moving away from the MAC (Figure 36- C, D) which signifies the initiation of MIC division through mitosis. The signal was consistently present after the MIC had completed mitosis (Figure 36-E). Two useful insights can be drawn from this analysis. First, Hat2/Cac $3^{\text {Tt }}$ functions in a cell cycle dependent manner. Second, during interphase when cell is not dividing Hat $2 / \mathrm{Cac} 3^{\mathrm{Tt}}$ might not be required in the MIC as $\mathrm{Cac}_{2}{ }^{\mathrm{Tt}}$ interacting protein. Additionally, consistent with its role as Hat2 subunit of Hat1-complex, a relatively weak localization was also observed in the cytoplasm of cells undergoing MIC and MAC division. 

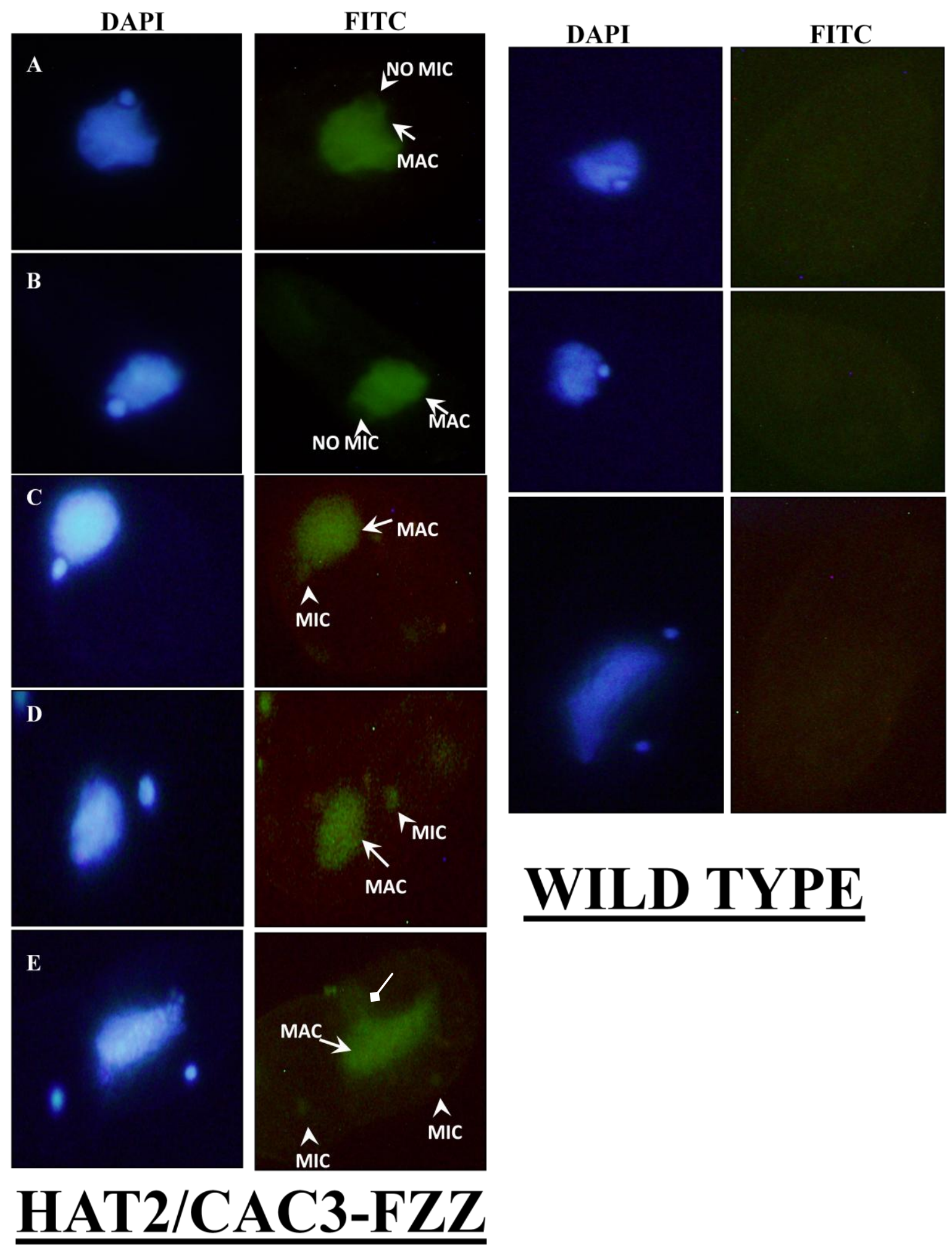

Figure 36: Indirect immunofluorescence of Hat2/Cac3 ${ }^{\mathrm{Tt}}$-FZZ. Hat $2 / \mathrm{Cac} 3^{\mathrm{Tt}}$ localization is temporally correlated with mitosis in logarithmically growing T.thermophila (A-E). Cells were stained with DNA-specific dye DAPI to capture the nuclei orientation whereas antiFLAG antibody was used to identify Hat $2 / \mathrm{Cac} 3{ }^{\mathrm{Tt}}$ localization. Arrow heads denote the position of MIC whereas open arrows indicate the MAC location. Box pointer denotes possible cytoplasmic localization in dividing cells. 


\section{Chapter 4: Discussion}

How does a eukaryotic cell assemble its chromatin into distinct euchromatic and heterochromatic states is one of the long standing questions in modern molecular biology. Previous work by many laboratories have shown that CAF1 and HIR protein complexes are responsible for RD and RI chromatin assembly, respectively (Hoek and Stillman 2003; Tang

et al. 2006). In addition, diacetylation of newly synthesized H4 has also been related to chromatin assembly (reviewed in Parthun 2007). Most of the molecular analyses performed on these protein complexes are restricted to higher eukaryotes such as fungi and humans. Information regarding the function and composition of chromatin assembly protein complexes is limited outside of Opisthokonta lineages e.g. animals and fungi. The spatial distinction of two chromatin states in T. thermophila into distinct nuclei, i.e. silent chromatin in the form of MIC and transcriptionally active chromatin in the form of MAC, provides a unique opportunity to study the molecular processes related to their assembly. To extend on previous findings and gain additional insights I have presented here evidence regarding the composition of major chromatin assembly complexes in T. thermophila. This has allowed deriving useful insights regarding the functional links between chromatin assembly pathways and formation of distinct chromatin states within MIC and MAC.

\section{2- Nrp1 functions in $\mathrm{H} 3 / \mathrm{H} 4$ transport pathway}

NASP proteins are characterized by the presence of four TPR motifs where the second TPR is typically interrupted by a stretch of acidic residues (Dunleavy et al. 2007). The detailed structural analysis of Nrp1 indicates that the TPR motif organisation is consistent with NASP family proteins (Figures 7 and 8). Recently, a crystal structure of budding yeast Hif1 was reported (Liu et al. 2014). The reported structure indicates that Hif1 adopts a groove-like surface and acidic residues interrupting TPR2 which are crucial for 
H3/H4 binding (Liu et al. 2014). Interestingly, the de novo predicted structure of Nrp1 in considerably similar to the reported Hif1 structure. For example, the acidic residues in the TPR2 also form a groove-like surface. I hypothesize that this negatively charged pocket can provide a binding surface for positively charged histones $\mathrm{H} 3 / \mathrm{H} 4$, consistent with what has been shown in Hif1 structure (Liu et al. 2014).

NASP family of histone chaperones has been implicated in a variety of chromatin related processes including transport of newly synthesized histones $\mathrm{H} 3 / \mathrm{H} 4$ where they present these histones to Hat1-complex in the cytoplasm for $\mathrm{H} 4$ diacetylation and subsequently hand them over to Asf1 (Campos et al. 2010). NASP and Asf1 physical interaction has been shown in humans as well as in budding yeast (Fillingham et al. 2008; Campos et al. 2010; Alvarez et al. 2011). Recently, our laboratory showed that in $T$. thermophila Asf1 co-purifies with Nrp1, Imp $\beta 6$ and two novel Aip1/2 proteins (Garg et al. 2013). Consistent with what has been shown in humans and budding yeast, we proposed that Asf $1^{\mathrm{Tt}}$ - Imp $\beta 6$ function together to transport newly synthesized H3/H4 into both the MIC and MAC (Garg et al. 2013).In this regard, Aip1 and Aip2 appear to be protist specific proteins without any apparent homologs in higher eukaryotes as assessed by BLASTP searches. Considering their stable interactions with $\operatorname{Imp} \beta 6$ and Asf1, it is possible that they have a regulatory role in the transport of histones $\mathrm{H} 3 / \mathrm{H} 4$, analogous to that of human Codanin-1 (See below- Future guidelines). The proteomic data presented here reciprocally verifies Nrp1 physical interaction with Asf $1^{\mathrm{Tt}}$. This observation in turn suggests that NASP-Asf1 physical interaction is ancient and was already present well before the emergence of modern Opisthokonts. NASP interaction with heat shock proteins has been shown to function upstream of Asf1 in the H3/H4 supply chain (Campos et al. 2010). The observation that Nrp1 also co-purifies with two putative heat shock proteins provides support to the hypothesis that 
the $\mathrm{H} 3 / \mathrm{H} 4$ transport chain is conserved in $T$. thermophila. To extend on our previously proposed model (Garg et al. 2013) I suggest that HSP70-Nrp1 function in the transport of H3/H4 upstream of Asf $1^{\mathrm{Tt}}$ - Imp $\beta 6$ (See Figure 37), similar to what has been shown in humans (Campos et al. 2010). In contrast to human NASP and budding yeast Hif1, T. thermophila Nrp1 did not co-purify with Hat1/Hat2. It could possible represent transient interaction of Nrp1 with Hat1-complex mediated by histones $\mathrm{H} 3 / \mathrm{H} 4$. It will be useful to study $\mathrm{H} 3 / \mathrm{H} 4$ protein-protein interactions by $\mathrm{AP}-\mathrm{MS}$ in order to gain addition insights regarding the composition of histone shuttling complexes (Ashraf K. Fillingham J. and Pearlman RE, un-published).

The localization analysis indicates that Nrp1 is primarily a nuclear protein. While it predominantly localizes to the MAC and is not found in interphase MIC, MIC localization is observed during MIC nuclear division during vegetative growth. The MIC localization is consistent with proposed function of Nrp1 in histone supply chain. During replication new histones are required for deposition onto either newly replicated or parental DNA (Tagami et al. 2004). Thus localization to the MICs undergoing mitosis provides additional support to the proposed function of Nrp1 in $\mathrm{H} 3 / \mathrm{H} 4$ transport pathway. NASP expression has been shown to be a cell cycle regulated in humans (Richardson et al. 2000). Its over expression delays cell cycle progression from G1/S border (Alekseev et al. 2003). The temporal correlation of Nrp1 localization to mitotically dividing MICs suggests that similar to human NASP, it is also a cell cycle regulated protein.

Human NASP also functions to fine-tune soluble reservoirs of H3/H4 (Cook et al. 2011). Additionally, in vivo it can also bind with histone H1(Alekseev et al. 2005; Richardson et al. 2006) and in vitro can facilitate chromatin assembly (Finn et al. 2008). Although conclusive evidence is lacking but in vitro analysis indicates that human NASP is 
capable of assembling the nucleosomes from free histones (Osakabe et al. 2010). In order to facilitate transcription chromatin has to be transiently disassembled ( $\mathrm{Li}$ et al. 2007), thus suggesting a role for Nrp1 in chromatin assembly. For example, Nrp1 localization to the transcriptionally active MAC suggests that it might be involved more directly in regulating the transcription related processes presumably via histone storage and nucleosome formation activities. In addition, MAC localization as well as MIC localization during mitosis suggests that Nrp1 might be important for histone H3/H4 supply during RD and RI chromatin assembly processes.

Human NASP has several predicted and experimentally determined phosphorylation sites (reviewed in Finn et al. 2012). Experimental high throughput proteomic data suggests that during DNA damage at least $35 \%$ of these sites are phosphorylated by ataxia telangiectasia mutated (ATM)/ataxia telangiectasia and Rad3 related (ATR), DNA-dependent protein kinase (Matsuoka et al. 2007; Dephoure et al. 2008). Recently T. thermophila 'Phosphoproteome' database was published (Tian et al. 2014). T. thermophila samples collected from three different physiological/developmental stages including vegetative growth, starvation and conjugation were pooled together and were subjected to MS-based global phosphoproteomic analysis (Tian et al. 2014). Interestingly, analysis of Nrp1 using phosphoproteome database indicated that it is phosphorylated at serine $178 / 180$. The exact function of these phosphorylation events is not known however considering the circumstantial evidence from human NASP phosphorylation profile I speculate that Nrp1 has a role downstream of DNA repair seemingly via histone supply and chromatin assembly related activities. 


\section{Cytoplasm}

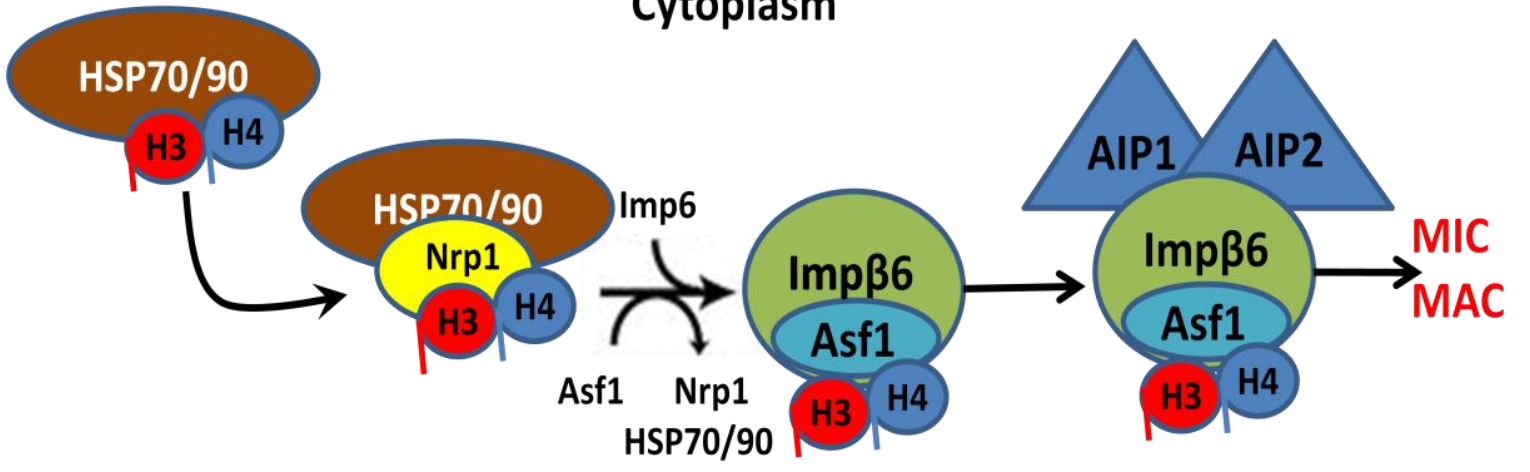

Figure 37: Model for the transport of newly synthesized histones $\mathrm{H3} / \mathrm{H} 4$ in $T$. thermophila. This model is an update to Garg et al. 2013. As an addition, heat shock proteins are proposed to function upstream of Asf1 in this pathway.

\section{3- Hira is conserved in T. thermophila}

Hira family proteins are responsible for the deposition of histone $\mathrm{H} 3$ variants (H3.2/H3.3) in a RI manner (Tagami et al. 2004; Tang et al. 2006; Latreille et al. 2014). In T. thermophila, HHT3 and HHT4 genes encode $\mathrm{H} 3.3$ and $\mathrm{H} 3.4$ variants, respectively. To learn about the deposition pathway of these H3 variants, a putative $H I R A^{T t}$ gene was identified through BLAST searches using human Hira as a query. Domain and sequence alignment analyses indicated that Hira $^{\mathrm{Tt}}$ is well conserved at amino acid as well as at structural level (See Figure 13). Consistent with human Hira and budding yeast Hir1 proteins, Hira ${ }^{\mathrm{Tt}}$ possesses at least five WD40 repeats. The physical interaction between Hira and Asf1 has been well documented in humans and budding yeast and is mediated via a small sequence motif called B-domain (Green et al. 2005; Tang et al. 2006; Fillingham et al. 2009). Through MSA analysis I predicted the existence of a B-domain in Hira ${ }^{\mathrm{Tt}}$. Furthermore, I also found that Hira ${ }^{\mathrm{Tt}}$ putative B-domain is highly conserved and the identity of amino acid residues essential for Asf1 interaction in humans (Tang et al. 2006) has almost remained unchanged. These observations suggest that Hira-Asf1 interaction might be conserved in $T$. thermophila - a relatively divergent eukaryote. Hira proteins have been suggested to form Nand C-termini $\beta$-propellers (Balaji et al. 2009), albeit no structural data is available till date. 
The predicted structure of Hira ${ }^{\mathrm{Tt}}$ indicates that $\mathrm{N}$-terminal $\beta$-propeller is present however the C-terminal region adopts an extended topology with several alpha helices packaging against each other. Nevertheless, the middle region of the protein shows a $\beta$-propeller-like fold suggesting the present of second possible $\beta$-propeller.

The MS analysis of Hira ${ }^{\mathrm{Tt}}$-FZZ successfully recovered the bait; however, no other protein was detected. There could be several reasons for this apparent discrepancy. First, Hira $^{\mathrm{Tt}}$ interaction with Asf1 ${ }^{\mathrm{Tt}}$ (or other proteins) could be very transient. Such transient interactions could easily be lost during purification procedure due to harsh experimental conditions such as those used in this study including high salt concentration $(350 \mathrm{mM} \mathrm{NaCl})$. Second, insertion of C-terminal FZZ tag could potentially interfere with protein proper folding and hence its interactions. To circumvent these limitations, the purification procedure should be carried out at relatively low salt conditions for capturing transient interactions, albeit extremely low salt concentration (e.g. 50mM) may not lyse the nuclei. Additionally, Nterminal tagging might be used as an alternative to C-terminal tag. My gene network analyses of Hira ${ }^{\mathrm{Tt}}$ and human Hira indicated that both genes/proteins have very similar network connections. For example, Asf1 as well as subunits of transcriptional regulator FACT complex are found in both Hira ${ }^{\mathrm{Tt}}$ and human Hira networks. The observation that $\mathrm{Asf}^{\mathrm{Tt}}$ is found in Hira ${ }^{\mathrm{Tt}}$ expression network suggests that these proteins might be functionally linked. This conclusion sounds reasonable due to: 1) - the presence of putative B-domain in Hira ${ }^{\mathrm{Tt}}$ 2)- extensive amount of literature exists on Hira-Asf1 interaction in other organisms (Sharp et al. 2005; Song et al. 2013; Latreille et al. 2014).

Hira proteins have been linked to transcription-related RI chromatin assembly (reviewed in De Koning et al. 2007). Thus the observed Hira ${ }^{\mathrm{Tt}}$ localization to transcriptionally active MAC is in agreement with the general role of Hira proteins. Based on 
Hira $^{\mathrm{Tt}}$ MAC localization and functional link with $\mathrm{Asf} 1^{\mathrm{Tt}}$ that is found in the expression network, I propose that these two proteins function together to deposit histones onto transcriptionally active MAC chromatin. Hira ${ }^{\mathrm{Tt}}$ signal observed in the MIC possibly represent its additional functions in the general maintenance of silent chromatin. It has been shown that physically interacting Asf1 and Hir1 proteins in budding yeast function together to promote heterochromatic gene silencing (Kaufman et al. 1998; Sharp et al. 2001; Prochasson et al. 2005). Interestingly $\mathrm{Asf}^{\mathrm{Tt}}$ also predominantly localizes to the MIC in vegetative cells which mimics to the localization pattern of $\mathrm{Cac}^{\mathrm{Tt}}$ as noted above. Hira ${ }^{\mathrm{Tt}}$ signal in the MIC and Asf $1^{\mathrm{Tt}}$ localization pattern suggests that both of these proteins might be involved in chromatin general maintenance as well as gene silencing in the MIC. Additionally, budding yeast Hir1 proteins as well as CAF1 complex both have been shown to be essential for proper kinetochore assembly. Interestingly in T. thermophila only MIC genome has centromeres whereas MAC lacks these structures. Thus MIC localization during DNA replication could also represent Hira $^{\mathrm{Tt}}$ involvement in the proper chromosomal segregation (Sharp et al. 2002). Finally, Hira $^{\mathrm{Tt}}$ presence in the MIC correlates well with $T$. thermophila H3.3 which has also been shown to faintly localize in the MIC (Cui et al. 2006).

\section{4- Deposition-related histone acetylation in T. thermophila}

During early stages of $T$. thermophila conjugation newly synthesized histones are exclusively deposited into dividing MICs (Allis and Wiggins 1984). Early studies have shown that newly synthesized histones H3 and H4 are heavily acetylated (Allis et al. 1985). Since MIC is transcriptionally inert therefore these acetylations were interpreted as deposition or chromatin assembly related PTMs (Allis et al. 1985). Surprisingly, despite the identification of these deposition-related histone marks the enzyme that catalyses these reactions has not been studied in $T$. thermophila. Studies in budding yeast have shown that 
deposition related acetylation marks are carried out by Hat1 enzyme (reviewed in Parthun 2012). T. thermophila genome encodes six putative Hats, Hat ${ }^{\mathrm{Tt}}$ being one of them, as indentified through BLAST searches (Fillingham et al. un-published).

My MSA analysis suggested that putative Hat $1^{\mathrm{Tt}}$ is a highly conserved protein with significant homology to the other well characterized family members. The structural prediction indicates that the core of $\mathrm{Hat}^{\mathrm{Tt}}$ can be superimposed with human and budding yeast Hat1 enzymes. These observations suggest that the putative Hat $1^{\mathrm{Tt}}$ belongs to type-B HATs family that come under the umbrella of GNAT super-family. The localization analysis indicated that $\mathrm{Hat}^{\mathrm{Tt}}$ is predominantly a MAC protein. The deposition-related histone acetylation has been well documented as a cytoplasmic event (Allis et al. 1985; Sobel et al. 1995), and consistently evidence was found for Hat ${ }^{\mathrm{Tt}}$ cytoplasmic localization. In the light of existing literature for $T$. thermophila deposition-related histone acetylations (Allis et al. 1985; Sobel et al. 1995) and known cytoplasmic functions of Hat1-complex in budding yeast (Ruiz-García et al. 1998; for review Parthun 2012), it is possible that Hat $1^{\mathrm{Tt}}$ localization might be due to deposition-related acetylation of newly synthesized histones $\mathrm{H} 3 / \mathrm{H} 4$. In budding yeast Hat1 initially was described as cytoplasmic protein based on the fact that it acetylated nucleosome free histones H4 (Parthun et al. 1996). However subsequent studies showed that it also localizes to nucleus with strong affinity for histones H4 (Ruiz-García et al. 1998). Some of the described nuclear roles of budding yeast Hat1 include DNA-repair linked chromatin assembly (Ge et al. 2011) and telomeric silencing (Mersfelder and Parthun 2008). Thus it is possible that similar to budding yeast, Hat $1^{\mathrm{Tt}}$ also has a role in chromatin assembly related with DNA repair mechanisms. Furthermore, it has been shown that $T$. thermophila HDAC called Thd2 functions in deposition-related deacetylation in the MIC 
(Smith et al. 2008). Thus Hat $1^{\mathrm{Tt}}$ MIC localization is consistent with the fact that newly synthesized histones are transiently acetylated before deposition onto DNA.

T. thermophila is rich in transcription-related histone acetylation (Brownell et al. 1996) for example H3K56ac (Garcia et al. 2007). In humans H3K56ac is catalyzed by two HATs, p300/CBP (Das et al. 2009) and hGcn5(Tjeertes et al. 2009) as well as by Asf1a (Das et al. 2009). Although, one of the earliest HAT activities ever reported were from $T$. thermophila Gen5 (Brownell et al. 1996), however an in vivo direct functional link between H3K56ac and any known HATs in T. thermophila has not been established yet. Considering the MAC localization it is tempting to speculate that Hat $1^{\mathrm{Tt}}$ might be catalyzing H3K56ac.

\section{5- T. thermophila Hat1 and Caf1 complexes share Hat2/Cac3 subunit}

In budding yeast and humans, Hat1 is known to form a complex with Hat2 (RbAp46 in humans) protein (reviewed in Parthun 2012). Hat2 has been shown to strongly enhance the catalytic activity of Hat1 by increasing its interaction with histone H4 (Ruiz-García et al. 1998). Interestingly, the residues shown to be responsible for Hat2 interaction (Li et al. 2014) appear highly conserved in $\mathrm{Hat}^{\mathrm{Tt}}$. In accordance with these observations, proteomic analysis of Hat $1^{\mathrm{Tt}}$ indicated that it co-purifies with another protein that shares significant homology to Hat2 (RbAp46). This suggested that the composition of Hat1-complex in $T$. thermophila is conserved. Previous studies have shown that Hat2 (RbAp46) is a WD40 repeat protein and shares high degree of similarity with Cac3 (RbAp48 in humans) subunit of the CAF1 complex (Murzina et al. 2008). These two proteins are encoded by two separate genes in higher eukaryotes (Murzina et al. 2008; Li et al. 2014). Interestingly, analysis of $\mathrm{Cac} 2^{\mathrm{Tt}}$ interacting proteins indicated that the above mentioned putative Hat $2^{\mathrm{Tt}}$ also purifies as $\mathrm{Cac} 3^{\mathrm{Tt}}$ subunit of putative $\mathrm{CAF} 1^{\mathrm{Tt}}$ complex. This indicates that Hat $2 / \mathrm{Cac} 3^{\mathrm{Tt}}$ functions in the context of two separate and distinct protein complexes, i.e. $\mathrm{CAF} 1^{\mathrm{Tt}}$ and $\mathrm{Hat} 1^{\mathrm{Tt}}$. One 
explanation for this observation could be that functions distributed between two separate genes in higher eukaryotes are carried out by a single gene in T. thermophila. I hypothesize that $H A T 2 / C A C 3^{T t}$ is an ancestral gene of two paralogous genes i.e. HAT2 and $C A C 3$ that are found in higher eukaryotes. Based on the proteomic evidence at hand, I further propose that after gene duplication the ancestral functions of $H A T 2 / C A C 3$ were distributed between $H A T 2$ and $C A C 3$ through a sub-functionalization event.

\section{6- T. thermophila Caf1 interacts with CKII complex}

The three subunit CAF1 complex has been shown to promote nucleosome assembly onto replicating DNA (Smith and Stillman 1989; Verreault et al. 1996). The Cac2 or p60 subunit of CAF1 complex harbours B-domain required for interaction with Asf1 for receiving newly synthesized histones $\mathrm{H} 3 / \mathrm{H} 4$ (Malay et al. 2008). The $T$. thermophila genome encodes a putative Cac 2 protein that is well conserved as assessed by sequence and domain analyses. MSA analysis indicated that B-domain is also present in the putative $\mathrm{Cac} 2^{\mathrm{Tt}}$ suggesting a possibility of interaction with $\mathrm{Asf} 1^{\mathrm{Tt}}$. Cac2 proteins have been predicted to make only one $\beta$-propeller structure (Balaji et al. 2009), possibly due to loss of C-terminal WD40 repeats. It is notable however that crystal structure for any of Cac2 proteins has not been reported yet. Interestingly, the predicted $\mathrm{Cac} 2^{\mathrm{Tt}}$ structure indicated that it forms two distinct $\beta$-propellers each with 5-blades. Although only 7 - or 8 - bladed $\beta$-propellers have been structurally confirmed thus far (Xu and Min 2011), however none-WD40 proteins have been shown to contain less number of blades (Paoli 2001). The predicted structure of Cac $2^{\mathrm{Tt}}$ in this regard offers several attractive features. For example, it can be hypothesized that the second $\beta$-propeller in $\mathrm{Cac} 2^{\mathrm{Tt}}$ may provide additional scaffold to assemble protein complexes.

AP-MS data indicated that $\mathrm{Cac} 2^{\mathrm{Tt}}$ co-purifies with two proteins that share significant homology to Cac1 and Cac3 subunits of CAF1 complex (also see above for Hat2/Cac3 ${ }^{\mathrm{Tt}}$ ). 
CAF1 has been suggested to be evolutionarily conserved protein complex that functions in RD chromatin assembly (Balaji et al. 2009). The work presented here is the first experimental characterization of CAF1 complex in a divergent unicellular eukaryotic lineage i.e. ciliates. Interestingly, AP-MS data also revealed that $\mathrm{Cac} 2^{\mathrm{Tt}}$ co-purifies with three putative subunits of CKII complex. The CKII is an evolutionarily conserved serine/threonine protein kinase (Meggio and Pinna 2003). These interactions suggest that $\mathrm{CAF} 1^{\mathrm{Tt}}$ might be a target of CKII mediated phosphorylation. Alternatively, CAF1 might be involved in guiding the CKII to target the histone for phosphorylation. CAF1 has previously been shown to be highly phosphorylated at Cac1 (p150) and Cac2 (p60) subunits (Smith and Stillman 1991) with several predicted CKII sites (Ridgway and Almouzni 2000). In vitro studies have demonstrated that CAF1 function can be regulated by cell-cycle kinases/ phosphatases (Martini et al. 1998) and phosphorylation of Cac1 subunit results in its dimerization (Quivy et al. 2001). Furthermore, in vitro Cac1 can be phosphorylated by cell division cycle-7 (Cdc7) kinase (Gérard et al. 2006). This phosphorylation favours Cac1 interaction with PCNA and recruitment to sites of double-stranded DNA breaks (Gérard et al. 2006). The data presented here is the first in vivo evidence of CAF1-CKII interaction. Interestingly, both Cac1 and Cac2 subunits have predicted CKII target sites that appear be highly conserved from $T$. thermophila to humans (see Future guidelines). These results suggest that CKII mediated CAF1 phosphorylation might be physiological important for its proper functioning.

\section{7 - Functional insights into CAF1 ${ }^{\mathrm{Tt}}$ complex}

It was recently shown (Garg et al. 2013) and also re-confirmed in this work that Asf $1^{\mathrm{Tt}}$ in growing cells localizes to both MAC and MIC with a stronger signal in the latter. The prediction that $\mathrm{Cac} 2^{\mathrm{Tt}}$ possesses a B-domain suggests that it might be functionally linked with $\mathrm{Asf}^{\mathrm{Tt}}$. Interestingly, IF of $\mathrm{Cac}^{\mathrm{Tt}}$ showed that its localization pattern significant 
overlaps with that of Asf ${ }^{\mathrm{Tt}}$. Similar to Asf $1^{\mathrm{Tt}}$, Cac2 $2^{\mathrm{Tt}}$ also localizes to both MAC and MIC with strong signal intensity in the MIC. I postulate that in growing cells both $\mathrm{Asf} 1^{\mathrm{Tt}}$ and $\mathrm{Cac} 2^{\mathrm{Tt}}$ function together to deposit newly synthesized histones H3/H4. Surprisingly, during vegetative growth when cells are not dividing Hat $2 / \mathrm{Cac}^{\mathrm{Tt}}$ signal was observed only in the MAC. This suggests that $\mathrm{Cac} 2^{\mathrm{Tt}}$ may have functions in the MIC that are independent of $\mathrm{Hat} 2 / \mathrm{Cac}^{\mathrm{Tt}}$. It is possible that Hat $2 / \mathrm{Cac}^{\mathrm{Tt}}$ is dispensable for the minimal $\mathrm{CAF} 1^{\mathrm{Tt}}\left(\mathrm{Cac} 1^{\mathrm{Tt}}\right.$ and $\mathrm{Cac} 2^{\mathrm{Tt}}$ ) complex required for chromatin assembly during interphase. This hypothesis would be consistent with the earlier observations that p150 and p60 subunits are sufficient for human CAF1 activity (Kaufman et al. 1995). To this end the localization analysis of putative $\mathrm{Cac}^{\mathrm{Tt}}$ would be informative.

Interestingly, in vegetative cells undergoing nuclear division, a relatively faint Hat $2 / \mathrm{Cac}^{\mathrm{Tt}}$ signal was observed in the MICs. As MIC starts moving away from the MAC in preparation of mitosis, a signal starts appearing in the MIC and remains until completion of the nuclear division. It is likely that a full three subunit $\mathrm{CAF} 1^{\mathrm{Tt}}$ complex is required in dividing cells to optimize the efficiency of RD chromatin assembly process.

\section{8- Hat2/Cac3 ${ }^{\mathrm{Tt}}$ is a multi-function protein}

The smallest subunit RbAp48 (or Cac3) of CAF1 complex which is highly homologous to RbAp46 (or Hat2) appears to be functionally more diverse than the larger subunits (Ridgway and Almouzni 2000) . For example, both proteins associate with the retinoblastoma protein 1, a tumor-suppressor protein involved in cell-cycle control (Bartek et al. 1997). Moreover, both proteins are component of SIN3 complex which acts as a critical transcriptional co-repressor (Grzenda et al. 2009). The domain and sequence analysis of Hat $2 / \mathrm{Cac}^{\mathrm{Tt}}$ indicates that it shares significant homology with both RbAp48 and RbAp46 proteins found in humans and other organisms. Structural prediction of Hat $2 / \mathrm{Cac}^{\mathrm{Tt}}$ indicates 
that similar to the structures of human RbAp46/RbAp48 (Dutnall et al. 1998) and budding yeast Hat2 (Li et al. 2014), it also forms a single $\beta$-propeller. In addition, the PP-loop shown to stabilize $\mathrm{H} 3 / \mathrm{H} 4$ interaction ( $\mathrm{Li}$ et al. 2014) is also well conserved in $\mathrm{Hat} 2 / \mathrm{Cac} 3^{\mathrm{Tt}}$ suggesting a structural platform for histone binding. Such degree of high structural conservation also predicts functional conservation. Proteomic analysis of $\mathrm{Hat} 2 / \mathrm{Cac} 3^{\mathrm{Tt}}$ indicated that in addition to $\mathrm{CAF} 1^{\mathrm{Tt}}$ and $\mathrm{Hat} 1^{\mathrm{Tt}}$, it forms stable complexes with several other proteins including putative $\mathrm{SIN} 3^{\mathrm{Tt}}, \mathrm{Rxt}^{\mathrm{Tt}}{ }, \mathrm{LIN} 9^{\mathrm{Tt}}$ and Tesmin/TSO1-like ${ }^{\mathrm{Tt}}$. SIN3 has been shown to form a large protein complex that in addition to RbAp46 and RbAp48 is composed of several other proteins including HDAC1/2, SAP18/30 and SDS3 (Grzenda et al. 2009). Rxt3 is a component of yeast Rpd3 HDAC complex which also contains SIN3 as its core subunit and functions to modulate the gene expression (Ruiz-Roig et al. 2010). Surprisingly, none of the 18 putative THDs co-purified with Hat $2 / \mathrm{Cac}^{\mathrm{Tt}}$ possibly accounting for an indirect interaction. For example, it is likely that THDs - Hat $2 / \mathrm{Cac} 3^{\mathrm{Tt}}$ interactions are not direct and rather are mediated by some other protein factors such as SIN3. Additional APMS experiments might help elucidating the involvement of any HDAC in this complex. Copurification of putative $\mathrm{SIN} 3^{\mathrm{Tt}}$ and $\mathrm{Rxt} 3^{\mathrm{Tt}}$ suggests that similar to human RbAp46/RbAp48, Hat $2 / \mathrm{Cac}^{\mathrm{Tt}}$ might be a component of HDAC complexes that participate in transcription regulation. Interestingly, it has been shown that CAF1 complex remains at the site of replication to co-ordinate chromatin maturation after the deposition of newly synthesized histones (Taddei et al. 1999). After RD chromatin assembly, the RbAp48 subunit can switch its interaction from CAF1 to one of its interacting deacetylase complexes to facilitate histone deacetylation (Roth and Allis 1996; Taddei et al. 1999). The physical interaction of Hat $2 / \mathrm{Cac} 3^{\mathrm{Tt}}$ with SIN $3^{\mathrm{Tt}}$ and Rxt $3^{\mathrm{Tt}}$ imply that it might as well be the case in $T$. thermophila. 
The suggestion that Hat $2 / \mathrm{Cac} 3^{\mathrm{Tt}}$ is functionally diverse is further supported by its interaction with putative LIN9 ${ }^{\mathrm{Tt}}$. LIN9 is a subunit of evolutionary conserved DREAM complex (Dp, RB-like, E2F and multi-vulval class B) (Esterlechner et al. 2013). The DREAM complex has been shown to be essential in regulating cell-cycle dependent gene expression in particular those genes required for G1/S transition (Sadasivam and DeCaprio 2013). Moreover, LIN9 has been shown to be essential for embryonic development and it has critical functions in tumour suppression via association with retinoblastoma protein (Reichert et al. 2010). The discovery that Hat $2 / \mathrm{Cac}^{\mathrm{Tt}}$ also interacts with $\mathrm{LIN}^{\mathrm{Tt}}$ suggests that Hat $2 / \mathrm{Cac}^{\mathrm{Tt}}$ might have a role regulating the cell-cycle. The human TESMIN gene was initially characterized as testes specific however subsequently its expression was also found in ovaries at specific stages (Sugihara et al. 1999; Olesen et al. 2004). It was also shown that mutant alleles of the Arabidopsis thaliana TSO1 gene (TESMIN homolog) were defective in flower and ovule development (Song et al. 2000). In Drosophila TESMIN homolog was found to regulate transcriptional activation in male germ lines and is a component of germline specific DREAM complex (Jiang et al. 2007). The fact that Hat $2 / \mathrm{Cac} 3^{\mathrm{Tt}}$ interacts with a putative TESMIN homolog in T. thermophila suggests important roles for this complex in the maintenance of germ line MIC chromatin.

Taken together, these data suggest that $\mathrm{Hat} 2 / \mathrm{Cac} 3^{\mathrm{Tt}}$ is a multifunction protein that might be critical for several chromatin related process including nucleosome assembly, gene transcription regulation as we as general maintenance of chromatin structure. It is clear that Hat $2 / \mathrm{Cac} 3^{\mathrm{Tt}}$ is a component of both $\mathrm{CAF} 1^{\mathrm{Tt}}$ and Hat $1^{\mathrm{Tt}}$ complexes however whether these two proteins compete for the same binding site on Hat $2 / \mathrm{Cac} 3^{\mathrm{Tt}}$ or they have distinct interacting sites is unclear. Furthermore, from the number of spectral counts-a semiquantitative measure of protein abundance in sample (Lundgren et al. 2010), it appears that 
$\mathrm{SIN} 3^{\mathrm{Tt}}$ and $\mathrm{Rxt} 3^{\mathrm{Tt}}$ are major Hat $2 / \mathrm{Cac} 3^{\mathrm{Tt}}$ containing complexes. Whether these are exclusive complexes or exist as one major transcription regulatory super-complex is not known. 


\section{Conclusion}

The objective of the study was to conduct a detailed proteomic analysis of major chromatin assembly proteins to gain insights regarding the formation of distinct MAC and MIC chromatin states in T. thermophila. Proteins including Hat1, NASP, Asf1, Cac2, and Hir1 have been shown in humans as well as in budding yeast to be central to chromatin assembly pathways (for review see De Koning et al. 2007). Expression profiles of their respective homologs in $T$. thermophila also suggest functional links among these proteins (Figure 38).

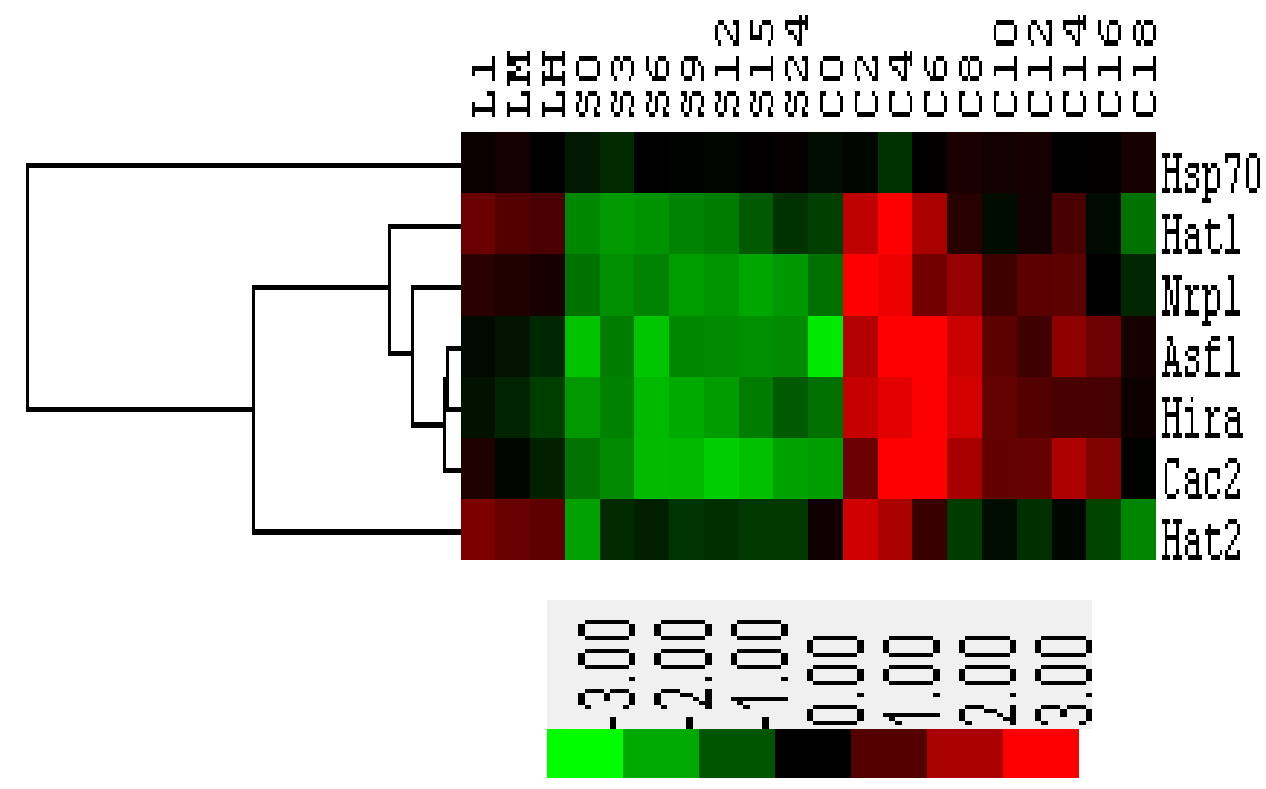

Figure 38: Hierarchal clustering of gene expression data of $T$. thermophila putative HAT1, NRP1, ASF1, HIRA AND CAC2. Gene expression data was acquired from publically available expression data base (see methods). Different growth conditions are indicated where $\mathrm{L}, \mathrm{S}$ and $\mathrm{C}$ represent vegetative, starvation and conjugation, respectively and numbers denote different time points at which samples were taken. Density key is also provided. The figure represents the fact that these genes can be clustered based on their expression profiles suggesting functional links among them. 
I have used AP-MS mediated discovery of protein-protein interactions to start defining the interactome of chromatin assembly complexes in T. thermophila (see Figure 39).

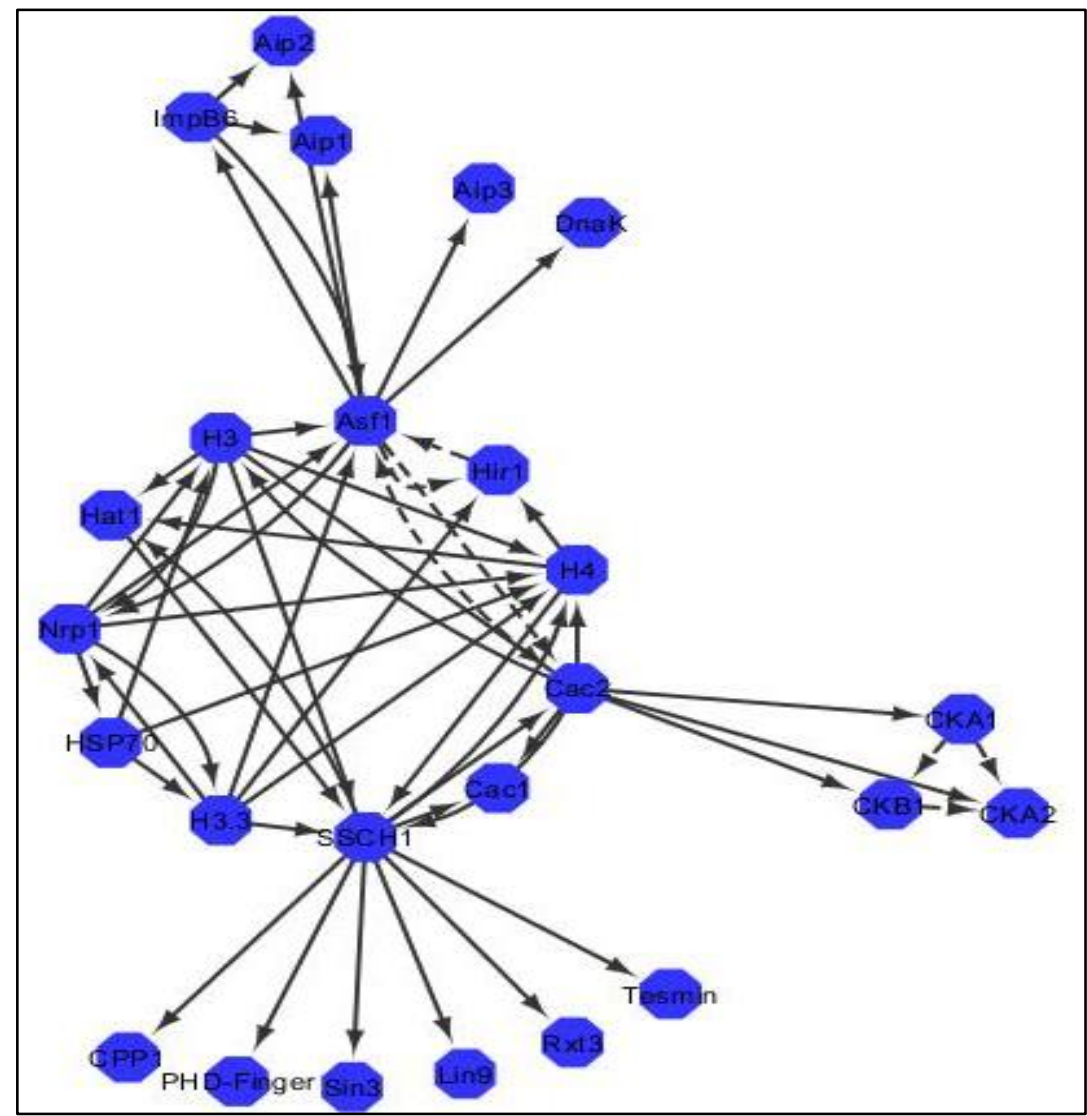

Figure 39: Protein-protein interaction network based on AP-MS data and expression profiles. Edges represent the direction of the interaction (Bait to prey). Double edges (arrows) represent reciprocal experimental verification of the interaction. Dotted arrows denote predicted interactions based on domain analysis (see text) and literature review. Also

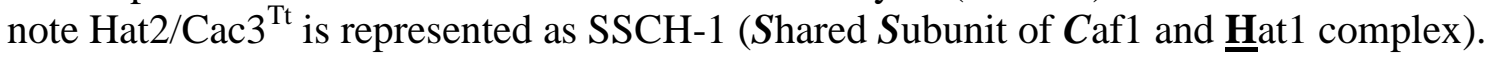

Using bioinformatics approaches I have established that RD and RI chromatin assembly complexes as well as histone modifying HAT1 complex are well conserved in this divergent eukaryote. Recently, we showed that $T$. thermophila $\mathrm{H} 3 / \mathrm{H} 4$ transport pathway involves Asf1 and Impß6 (Garg et al. 2013). I have updated this previously proposed model by reciprocally verifying Nrp1-Asf1 interaction as well as suggesting the involvement of heat shock proteins in this pathway. This hypothetical model of $\mathrm{H} 3 / \mathrm{H} 4$ transport is now significantly consistent with that of humans (see Campos et al. 2010) (also see Figure 37). 
My work has led to the first in vivo demonstration of CAF1-CKII physical interaction implicating CKII as the enzyme responsible for the CAF1 phosphorylation. In addition, protein-protein interaction data has firmly established that $\mathrm{CAF} 1^{\mathrm{Tt}}$ and $\mathrm{Hat} 1^{\mathrm{Tt}}$ share a single subunit suggesting an event of sub-functionalization for RbAp46/RbAp48 over the evolutionary course of these cancer-suppressing multi-functional proteins (see Figure 40).

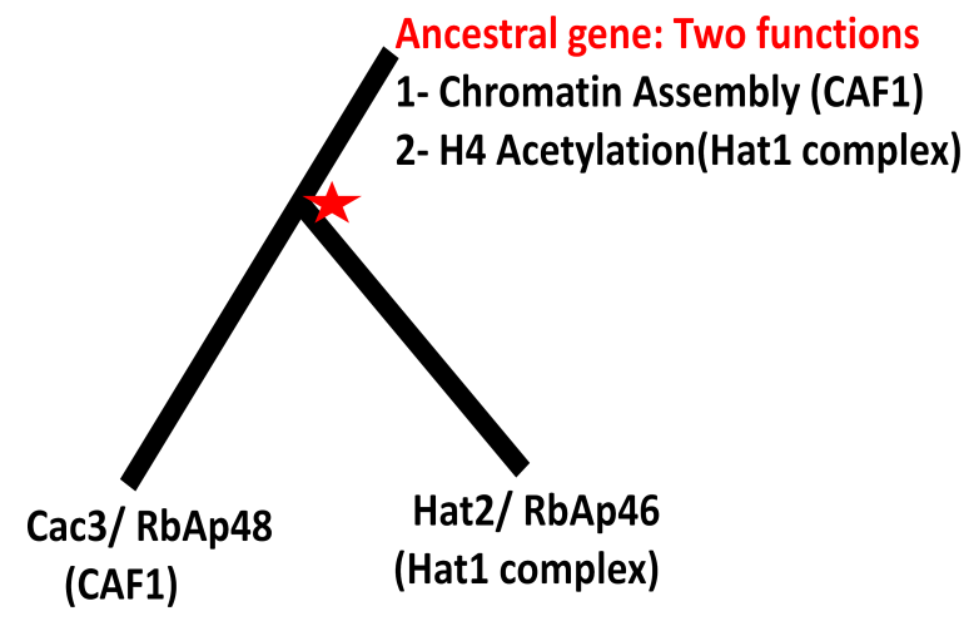

Figure 40: Hypothetical gene duplication resulting in division of functions. Ancestral functions are distributed between two paralog genes. Red star denoted the differentiation of gene functions after hypothetical duplication event.

In addition, localization patterns of $\mathrm{Cac}^{\mathrm{Tt}}$, $\mathrm{Hir} 1^{\mathrm{Tt}}, \mathrm{Asf1}^{\mathrm{Tt}}$ and $\mathrm{Hat} 2 / \mathrm{Cac} 3^{\mathrm{Tt}}$, serving as a circumstantial evidence, have provided novel mechanistic insights with respect to the connections between formation of distinct chromatin states and RD/RI pathways. For example, $\mathrm{Cac} 2^{\mathrm{Tt}}$ and $\mathrm{Hat} 2 / \mathrm{Cac} 3^{\mathrm{Tt}}$ localization patterns suggested that the latter is dispensable for CAF1 activity during resting stage of the cell cycle whereas it is indispensable during Sphase. Similarly, Cac $2^{\mathrm{Tt}}$, Hir $1^{\mathrm{Tt}}$, Asf $1^{\mathrm{Tt}}$ immunofluorescence analysis suggested that they are functionally linked and might have critical roles in the formation of transcriptionally active MAC chromatin as well as in the general maintenance of MIC. In addition, AP-MS of Hat $2 / \mathrm{Cac}^{\mathrm{Tt}}$ has revealed that it forms multiple complexes and might be involved in several chromatin related processes ranging from deposition related $\mathrm{H} 4$ acetylation, $\mathrm{RD}$ chromatin assembly, and deacetylation of histones to regulate the gene expression. Based on the data 
presented here, I propose that newly synthesized histones $\mathrm{H} 3 / \mathrm{H} 4$ are transported via evolutionary conserved pathway. Furthermore, I also propose that Asf1, CAF1 and Hir1 and Hat1-Hat2 proteins function in a co-operative manner to establish chromatin states within MIC and MAC (see Figure 41).

A

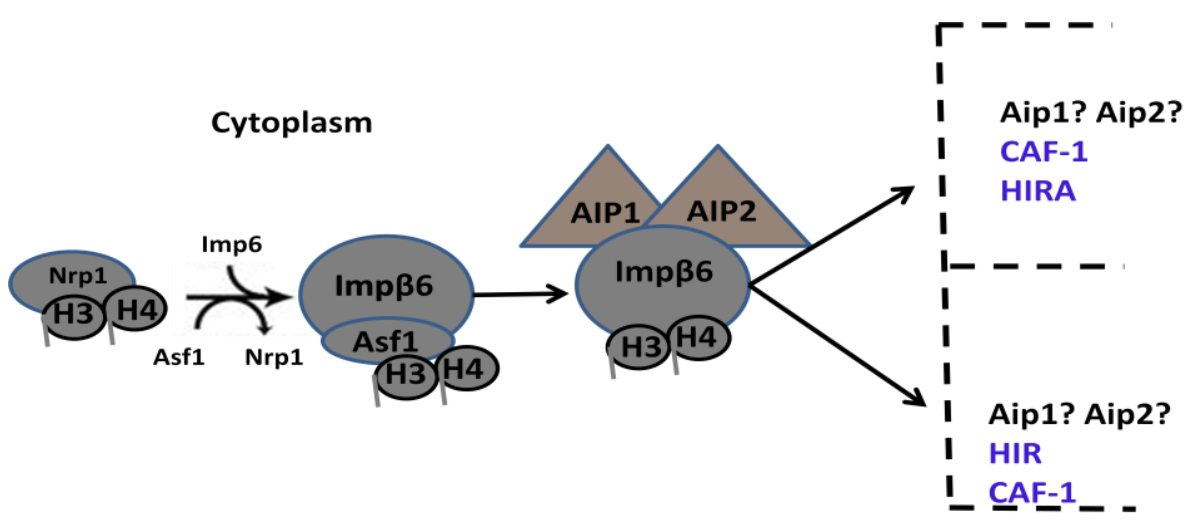

\section{B}

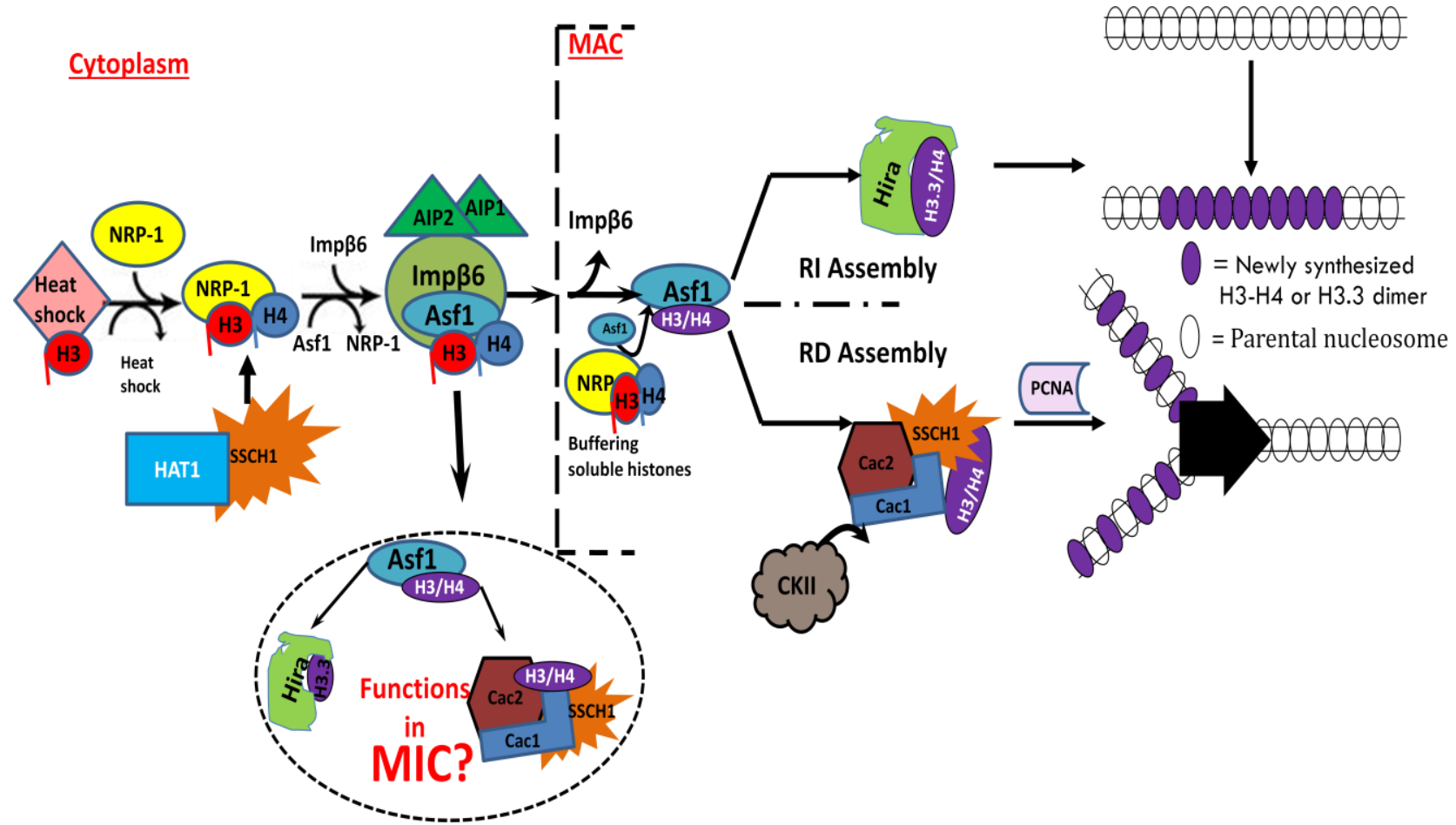

Figure 41: Updated version of $\mathrm{H3} / \mathrm{H} 4$ assembly pathway in $T$. thermophila. A: Adopted from Garg et al. 2013, proposed model of H3/H4 transport in T. thermophila. B: An updated version of the hypothetical model based on data presented in this report. Note: Minimum CAF1 version is shown in the resting stage MIC. See text for further details 


\section{Future guidelines and preliminary data}

The proposed model presented in figure 39 set paradigms for future research. However, it should be emphasized that histone $\mathrm{H} 3 / \mathrm{H} 4$ are central to this work and therefore proteomic characterization of these two proteins is essential for a better picture of $\mathrm{RD} / \mathrm{RI}$ chromatin assembly pathways in T. thermophila (Ashraf, K. Fillingham, J. and Pearlman, E. unpublished). Nevertheless, the proposed model offers several testable predictions as noted below.

1- The function of Aip1/2 is yet unclear. These proteins have been shown to physically interact with Asf $1^{\mathrm{Tt}}$ and Imp $\beta 6$ (Garg et al. 2013) . It is hypothesised in the model that these two proteins function in the cytoplasm to regulate the flow of $\mathrm{H} 3 / \mathrm{H} 4$ to the nuclei. To gain insights into possible functions of Aip1/2, it will be helpful to conduct a proteomic as well as sub-cellular localization analysis. To this end, I have engineered FZZ gene targeting vectors for both Aip1 and Aip2. In addition, $T$. thermophila cell lines expressing Aip1-FZZ from the native chromosomal loci have also been generated. The expression of tagged protein has been assessed by Western blots using anti-FLAG antibody (Figure $42 \mathrm{~A}$ ). In accordance with the proposed model, an indirect immunofluorescence analysis indicated that Aip1-FZZ exclusively localizes in the cytoplasm and not to any of the nuclei (Figure 42 B). Future work should focus on investigating the protein-protein interactions of Aip1/2 and subcellular localization of Aip2. In addition knock-out analysis of both of these genes will also be helpful to decipher their role in histones influx to the nuclei.

2- I have proposed that during interphase of cell cycle only a minimum CAF1 complex comprising Cac1 and Cac2 is present in the MIC. To this end, functional characterization of Cac1 will be informative. If it also localizes to both MAC and 
MIC during the interphase then it will establish that Cac1/Cac2 compose the minimum CAF1 in the interphase MIC. To accomplish this I have generated FZZ gene tagging vectors for targeting native chromosomal locus of $\mathrm{Cac}^{\mathrm{Tt}}$. In addition, Cac1 has been shown to have two PCNA interacting peptides (PIP1 and PIP2) (Rolef Ben-Shahar et al. 2009). My preliminary MSA analysis suggests that $\mathrm{Cac}^{\mathrm{Tt}}$ has conserved PIP sequence (See Figure 43). AP-MS would help in establishing the $\mathrm{Cac}^{\mathrm{Tt}}{ }^{\mathrm{PCNA}}{ }^{\mathrm{Tt}}$ interaction. Interestingly, MSA analysis suggested that $\mathrm{Asf1}{ }^{\mathrm{Tt}}$ also has a peptide that is very similar to the PIP sequence. The possibility of $\mathrm{Asf} 1^{\mathrm{Tt}}$ $\mathrm{PCNA}^{\mathrm{Tt}}$ interaction should be tested by generating PCNA-FZZ cell lines and conduction of AP-MS analyses.

A

B $\underline{\text { AIP1-FZZ }}$

Aip1 predicted Size: 260kDa FZZ-Tag: 18kDa Total predicted size: $278 \mathrm{kDa}$

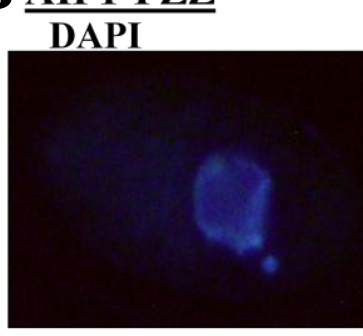
FITC
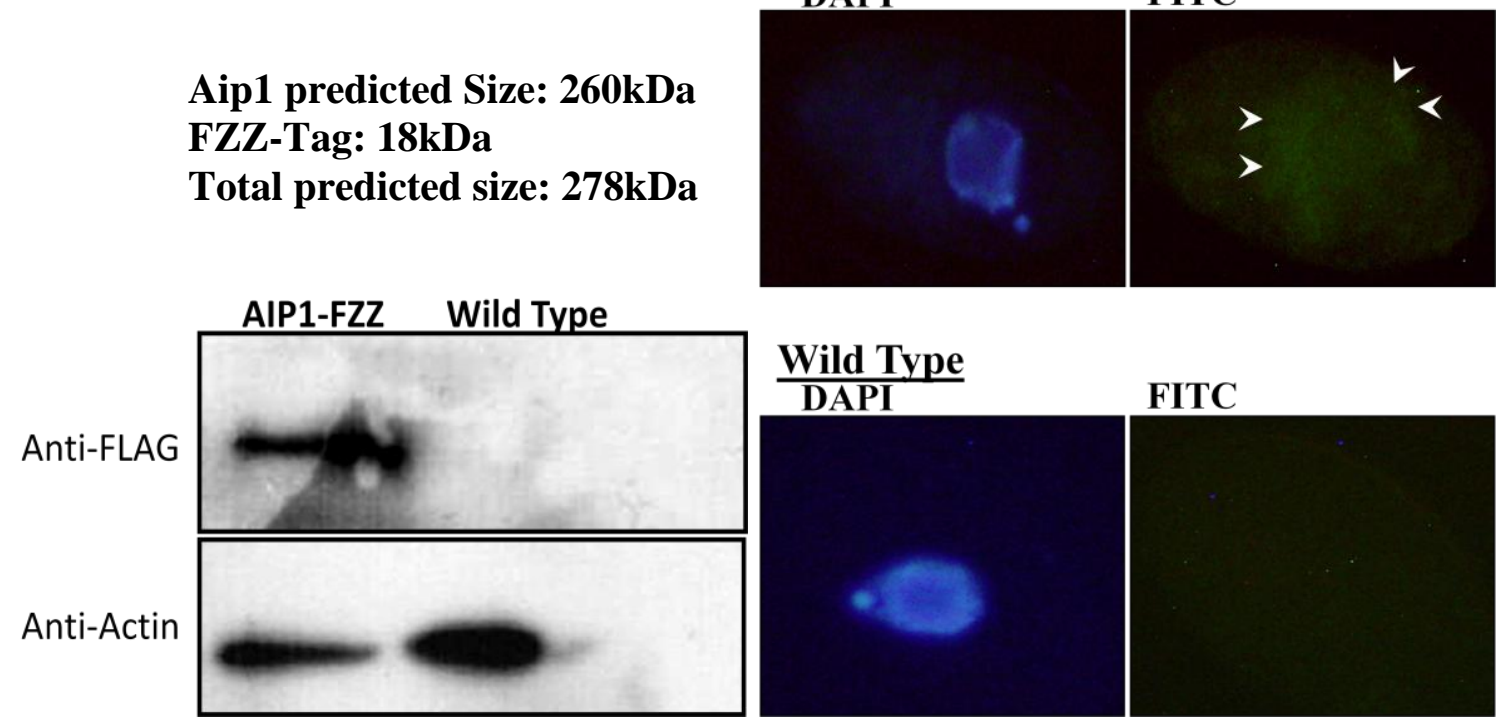

FITC

Figure 42: Expression of Aip1-FZZ assessed by Western blot and IF analysis. A: Successful expression of Aip1-FZZ (top panel) was detected with anti-FLAG antibody. Lower panel was probed with anti-actin as loading control. B: localization analysis of Aip1FZZ. Arrow heads indicate the Aip1 is concentrated at the periphery of MAC in the cytoplasm. 


$\begin{array}{cc}\text { PIP-Consensus/1-8 } & \text { Q-x x-h-x x-a a - } \\ \text { T. thermophila/1-9 } & \text { Q-KN-L-LS-FFA } \\ \text { H.sapiens-1/1-9 } & \text { K-AE-I-TR-FFQ } \\ \text { H.sapiens-2/1-7 } & \text { Q-AR-L-- P-FK- } \\ \text { S. cerevisiae/1-9 } & \text { Q-S R-I-GN-FFK } \\ \text { S. Pombe/1-9 } & \text { Q-LK-L-NN-FFT } \\ \text { Asf1 }{ }^{\mathrm{Tt}} / 1-9 & \text { Q-PR-I-TH-FN I }\end{array}$

Figure 43: Sequence alignment indicating PIP in various Cac1 proteins. The PIP consensus sequence is shown at the top where $\mathbf{h}$ represents residues with moderately hydrophobic side chains (e.g. L, I, M), a represents residues with highly hydrophobic, aromatic side chains (e.g. F, Y) and $\mathbf{x}$ is any residue. Note: H.sapiens Cac1 has two PIP sequences. Also Asf ${ }^{\mathrm{Tt}} \mathrm{PIP}$ like sequence is indicated.

3- $\mathrm{CAF}^{\mathrm{Tt}}-\mathrm{CKII}^{\mathrm{Tt}}$ interaction is shown in the model based on the fact that CKII copurifies with $\mathrm{Cac} 2^{\mathrm{Tt}}$-FZZ. Both $\mathrm{Cac} 1^{\mathrm{Tt}}$ and $\mathrm{Cac} 2^{\mathrm{Tt}}$ have consensus CKII phosphorylation sites as assessed by NetPhos 2.0 Server (http://www.cbs.dtu.dk/services/NetPhos/). Interestingly, these sites are highly conserved when compared with humans and budding yeast (See Figure 44). I have generated $\mathrm{CKA} 1^{\mathrm{Tt}}$ and $\mathrm{CKB} 1^{\mathrm{Tt}} \mathrm{FZZ}$ gene targeting vectors and a proteomic analysis of $\mathrm{CKII}^{\mathrm{Tt}}$ complex and investigation of sub-cellular localization will be helpful. It will be also helpful to assess the functional significance of this possible phosporylation event in budding yeast by mutational analysis. For example, budding yeast Cac1 and Cac2 CKII sites can be mutated followed by observing any phenotypic changes in chromatin environment.

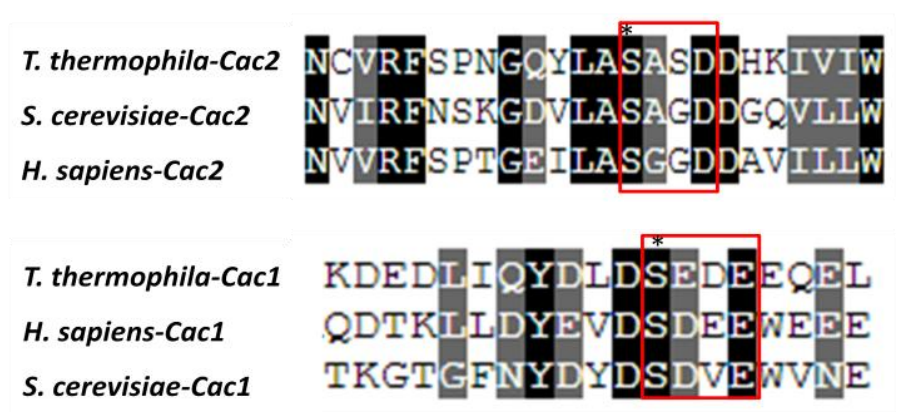

Figure 44: Predicted CKII sites on Cac2 and Cac1 protein. The red circle shows the conserved sequence whereas star indicates the predicted serine residue to be phosphorylated. 
4- Hat $2 / \mathrm{Cac}^{\mathrm{Tt}}$ co-purified with several potential histone deacetylase complexes including putative $\mathrm{SIN} 3^{\mathrm{Tt}}$ and $\mathrm{Rxt} 3^{\mathrm{Tt}}$. Functional significance of these interactions should be tested. For example, based on one AP-MS none of THDs co-purified with

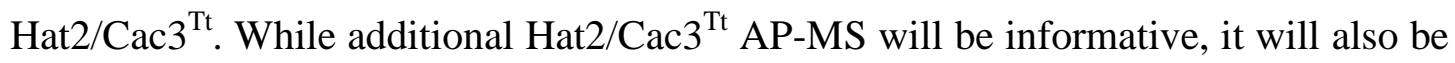
helpful to characterize SIN $3^{\mathrm{Tt}}$ and $\mathrm{Rxt} 3^{\mathrm{Tt}}$ and study their protein-protein interactions. Furthermore, KO analyses of these proteins would help revealing the functional significance. 


\section{Conserved Asf1-importin $\beta$ physical interaction in growth and sexual development in the ciliate Tetrahymena thermophila}

Jyoti Garg ${ }^{1}$, Jean-Philippe Lambert ${ }^{*, 2}$, Abdel Karsou ${ }^{*}, 3$, Susanna Marquez ${ }^{1}$, Syed NabeelShah $^{3}$,Virginia Bertucci ${ }^{1}$, Dashaini V. Retnasothie ${ }^{1}$, Ernest Radovani ${ }^{3}$, Tony Pawson ${ }^{2,4}$, Anne-Claude Gingras ${ }^{2,4}$, Ronald E. Pearlman ${ }^{1}$ and Jeffrey Fillingham $3,{ }^{\dagger 3}$

1. Department of Biology, York University, 4700 Keele St., Toronto M3J 1P3, Canada. 2. Samuel Lunenfeld Research Institute, Mount Sinai Hospital, 600 University Avenue, Toronto M5G 1X5, Canada.

3. Department of Chemistry and Biology, Ryerson University, 350 Victoria St., Toronto M5B 2K3 Canada.

4. Department of Molecular Genetics, University of Toronto, Toronto M5S 1A8, Canada.

* Equal contribution

$\uparrow$ To whom correspondence should be addressed: Jeffrey S. Fillingham, Department of Chemistry and Biology, Ryerson University, Toronto, ON, Canada, M5B2K3.

Tel.: 416-979-5000 x 2123; E-mail: jeffrey.fillingham@ryerson.ca

Contribution: In this article published in Journal of proteomics, I carried out structural Asf $1^{\mathrm{Tt}}$ structural analysis, performed tandem affinity purification once, participated in data analysis 


\section{Part 2: Molecular Evolutionary analysis of $\mathrm{H3} / \mathrm{H} 4$ chaperone NASP}

This section is adopted from research article originally published in BMC Evolutionary Biology (Nabeel-Shah et al. 2014). 


\section{Authors' contributions:}

SN-S retrieved the sequences, prepared sequence alignments, performed the molecular evolutionary analyses, conducted Bayesian phylogenetic analysis, and drafted the manuscript. KA performed ML and MP phylogenetic analyses, prepared the figures and conducted domain analysis using Pfam and PHYRE2. REP edited the manuscript and participated in design and co-ordination of the study and data analysis. JSF conceived the study, participated in its design and coordination, edited the manuscript and participated in data analysis. 


\section{Abstract:}

NASP is an essential protein in mammals that functions in histone transport pathways and maintenance of a soluble reservoir of histones $\mathrm{H} 3 / \mathrm{H} 4$. In humans, growing evidence implicates NASP miss-regulation in the development of a variety of cancers. Although a comprehensive phylogenetic analysis is lacking, NASP-family proteins that possess four TPR motifs are thought to be widely distributed across eukaryotes. Here we characterize the molecular evolution of NASP by systematically identifying putative NASP orthologs across diverse eukaryotic lineages ranging from kinetoplastids to those of the crown group. Our analysis suggests NASP is a conserved protein that was most likely present in the last eukaryotic common ancestor. We detected extensive silent divergence at the nucleotide level suggesting the presence of strong purifying selection acting at the protein level. We also observed a selection bias for high frequencies of acidic residues which we hypothesize to be

attributed to their critical function(s), further indicating the role of functional constraints operating on NASP evolution. Our data indicate that TPR1 and TPR4 constitute the most rapidly evolving functional units of NASP and may account for the functional diversity observed among well characterized family members. We also show that NASP paralogs that are found in ray-finned fish exist under differential genomic context and have undergone significant changes at the protein level suggesting some functional diversification in this lineage. In conjunction with previous reports regarding the evolutionarily conserved presence of proteins involved in $\mathrm{H} 3 / \mathrm{H} 4$ transport, we propose that the $\mathrm{H} 3 / \mathrm{H} 4$ transport pathway is highly conserved and most likely was present in the last eukaryotic common ancestor. 


\section{Chapter 1: Introduction}

The fundamental repeating unit of eukaryotic chromatin is the nucleosome that wraps a 146bp stretch of DNA around a histone octamer consisting of two of each of histone H2A, H2B, H3 and H4 (Luger et al. 1997). The transport of histones from the cytoplasm to the nucleus and their subsequent assembly into nucleosomes is mediated by a diverse set of proteins including histone chaperones that are classified into several families based on their binding specificities and sequence and structural similarities (De Koning et al. 2007). One group of histone $\mathrm{H} 3 / \mathrm{H} 4$ chaperones is the nuclear autoantigenic sperm protein (NASP) family also known as the N1/N2 family.

N1 and N2 ( 105 and $\sim 110 \mathrm{kDa})$ originally isolated from Xenopus laevis nuclear extracts are encoded by genes with identical DNA sequences (Bonner 1975; De Robertis et al. 1978). The differences in biochemical as well as immunological properties have not been explained and therefore they were designated as $\mathrm{N} 1 / \mathrm{N} 2$. The $\mathrm{N} 1 / \mathrm{N} 2$ is expressed in $X$. laevis oocytes and specifically binds histones $\mathrm{H} 3 / \mathrm{H} 4$, providing a mechanism for the storage of the soluble histones required for DNA replication in the early embryo (Kleinschmidt et al. 1985; Dilworth et al. 1987). NASP is the mammalian homolog of N1/N2 and was first described in rabbit testes as a highly autoantigenic protein which shares greater than $50 \%$ similarity to N1/N2 (Welch et al. 1990; Batova and O’Rand 1996). In mammals, NASP predominantly exists as two alternatively spliced isoforms; one is considerably longer than the other and is expressed in embryonic tissues and testis (tNASP) whereas the smaller version called the somatic NASP (sNASP) and is highly expressed in all dividing cells (Richardson et al. 2000). In humans the NASP gene is a $35.33-\mathrm{kb}$ in size and is composed of 15 exons. It has 26 predicted splice variants with at least 20 of them potentially are protein encoding (Finn et al. 2012). Only two of these variants (tNASP and sNASP) have been experimentally 
characterized and it has been shown that tNASP is a protein of 778 amino acids in length whereas sNASP lacks a region of 339 residues (Richardson et al. 2000).

NASP expression is tightly cell cycle regulated and an over-expression causes delay in cell cycle progression at the G1/S border (Richardson et al. 2000; Alekseev et al. 2003). NASP is an essential protein in mammals as its gene disruption results in early embryonic lethality in mice (Richardson et al. 2006). Previous studies have shown that human NASP copurifies with replication dependent and independent histones H3.1 and H3.3 respectively (Tagami et al. 2004). In human cells, newly synthesized histones H3.1/H4 are thought to successively pass through at least four distinct cytosolic complexes (Campos et al. 2010). In this context, NASP has been shown to be involved in accepting the histones from heat shock proteins, in the Hat1-dependent acetylation of $\mathrm{H} 4$, and subsequently handing over these histones to another histone chaperone, anti-silencing factor 1 (Asf1) through a physical interaction that has been shown to exist in humans and Saccharomyces cerevisiae (Fillingham et al. 2008; Campos et al. 2010; Alvarez et al. 2011).

NASP family proteins share conserved motifs, possessing four tetratricopeptide repeats (TPR) where the second TPR is typically interrupted by a large acidic domain (Dunleavy et al. 2007). The NASP structural organisation is conserved from fungi to mice forming the SHNi-TPR protein family that was named for three initially characterized members, Silencing in the middle of the centromere protein 3 (Sim3-Schizosaccharomyces pombe), Hatlp-interacting factor-1 (Hif1, S.cerevisiae), and NASP-interrupted TPR repeatsv(Dunleavy et al. 2007). The TPR motifs are 34 amino acid long amphipathic helices that form a helix-turn-helix arrangement and are thought to provide a structural scaffold for mediating protein-protein interactions (D’Andrea and Regan 2003). Different TPR motifs in human NASP show different binding affinities for either histone H3/H4 or H1 (Wang et al. 
2008; Wang et al. 2012). For example, the acidic patch present within TPR2 is critical for H1-binding whereas TPR4 mediates the interaction with core $\mathrm{H} 3 / \mathrm{H} 4$ histones (Wang et al. 2012). Evidence from sedimentation velocity and equilibrium experiments by analytical ultracentrifugation as well as size-exclusion chromatography has suggested that under physiological conditions sNASP exists as a dimmer that facilitates chromatin assembly (Finn et al. 2008). These studies suggest that NASP might be involved in multiple functions involving histone dynamics.

In addition to mammals, NASP homologs have been detected in several eukaryotic models such as S.cerevisiae (Hif1), S.pombe (Sim3) and Caenorhabditis elegans (NASP-1) (Dunleavy et al. 2007). In addition to their conserved motif arrangement, these proteins share functional similarity in that they generally demonstrate histone $\mathrm{H} 3 / \mathrm{H} 4$ chaperone activity (Finn et al. 2012). Despite this, some functional diversity does exist among NASP family proteins. For example, Hif1 physically interacts with Hat1 and Hat2 as part of the Hat 1 complex that functions in the acetylation of newly synthesized histone H4 (Ai and Parthun 2004; Poveda et al. 2004), whereas Sim3 appears to specifically function in deposition of the centromeric H3 variant (Dunleavy et al. 2007), and does not appear to physically interact with Hat1 (Tong et al. 2012; Kim et al. 2014). In addition to this, a recent report suggests that $\operatorname{Sim} 3$ also has a general role in chromatin maintenance and acts as an H3/H4 chaperone with some overlapping functional characteristics with Asf1 (Tanae et al. 2012). In C. elegans, NASP-1 has been implicated in female development through its interactions with histone deacetylase and TRA-4 proteins (Grote and Conradt 2006). Additionally, human NASP functions in the fine tuning of a soluble reservoir of histones H3/H4 by handing over excessive histone H3 and H4 to heat shock proteins (HSP90/HSC70) for chaperone mediated autophagy (Cook et al. 2011). 
In humans, NASP expression is significantly altered in a variety of cancers including those of the ovary and prostate (Ali-Fehmi et al. 2010; Alekseev et al. 2011; Ma et al. 2012). Despite the demonstrated direct/indirect roles of NASP in a wide range of cellular processes including DNA replication, cell proliferation, blastocyst development, stem cell proliferation, neural stem cell differentiation, and the pluripotency of human embryonic stem cells (reviewed in Finn et al. 2012), questions remain about the underlying mechanistic details of NASP function. Recently, we found that a NASP family protein, NASP-related protein 1 (Nrp1) co-purifies with Asf1 in the ciliate protozoan T. thermophila suggesting that the Asf1NASP physical interaction is evolutionarily conserved in eukaryotes (Garg et al. 2013). Molecular evolutionary analysis has the potential to provide useful insights into protein function as well as providing information about changes in interacting partners (Eisen 1998; Thornton and DeSalle 2000; Altenhoff and Dessimoz 2012). Molecular evolutionary analyses of the proteins involved in the transport of histones H3/H4 including HSP90, Asf1 and Importin $\beta$ have previously been reported and suggest that these proteins are highly conserved throughout the eukaryotes (O'Reilly et al. 2011)(Chen et al. 2006)(Daganzo et al. 2003). Although NASP has been suggested to be equally widely present among eukaryotes, a comprehensive phylogenetic analysis of NASP family proteins is lacking.

We carried out a detailed phylogenetic analysis of NASP family proteins. I found that NASP is conserved across all of the major eukaryotic lineages ranging from the excavata to the mammals suggesting that it was most likely present in the last eukaryotic ancestor (LECA). High acidic amino acid composition in the TPR2 interruption region has been maintained by the presence of strong selection which in turn has biased the nucleotide composition in the corresponding NASP gene. In addition, we also found that different TPRs are evolving at different rates with TPR1/4 evolving faster than TPR2/3. Finally, in 
conjunction with previous evolutionary analyses on Asf1, HSP90 and Importin $\beta$ proteins, we suggest that histones $\mathrm{H} 3 / \mathrm{H} 4$ are transported via an evolutionarily conserved pathway that most likely was present in the LECA. 
Molecular evolution of NASP and conserved histone H3/H4 transport pathway

Syed Nabeel-Shah ${ }^{1}$, Kanwal Ashraf ${ }^{2}$, Ronald E. Pearlman ${ }^{2}$ and Jeffrey Fillingham ${ }^{1, \dagger}$

1. Department of Chemistry and Biology, Ryerson University, 350 Victoria St., Toronto M5B 2K3Canada.

2. Department of Biology, York University, 4700 Keele St., Toronto M3J 1P3, Canada.

$\dagger$ To whom correspondence should be addressed

BMC Evolutionary Biology 2014, 14:139 doi:10.1186/1471-2148-14-139 


\title{
Molecular evolution of NASP and conserved histone $\mathrm{H} 3 / \mathrm{H} 4$ transport pathway
}

Syed Nabeel-Shah¹, Kanwal Ashraf², Ronald E Pearlman² and Jeffrey Fillingham ${ }^{1 *}$

\begin{abstract}
Background: NASP is an essential protein in mammals that functions in histone transport pathways and maintenance of a soluble reservoir of histones $\mathrm{H3} / \mathrm{H} 4$. NASP has been studied exclusively in Opisthokonta lineages where some functional diversity has been reported. In humans, growing evidence implicates NASP miss-regulation in the development of a variety of cancers. Although a comprehensive phylogenetic analysis is lacking, NASP-family proteins that possess four TPR motifs are thought to be widely distributed across eukaryotes.

Results: We characterize the molecular evolution of NASP by systematically identifying putative NASP orthologs across diverse eukaryotic lineages ranging from excavata to those of the crown group. We detect extensive silent divergence at the nucleotide level suggesting the presence of strong purifying selection acting at the protein level. We also observe a selection bias for high frequencies of acidic residues which we hypothesize is a consequence of their critical function(s), further indicating the role of functional constraints operating on NASP evolution. Our data indicate that TPR1 and TPR4 constitute the most rapidly evolving functional units of NASP and may account for the functional diversity observed among well characterized family members. We also show that NASP paralogs in ray-finned fish have different genomic environments with clear differences in their GC content and have undergone significant changes at the protein level suggesting functional diversification.

Conclusion: We draw four main conclusions from this study. First, wide distribution of NASP throughout eukaryotes suggests that it was likely present in the last eukaryotic common ancestor (LECA) possibly as an important innovation in the transport of $\mathrm{H} 3 / \mathrm{H} 4$. Second, strong purifying selection operating at the protein level has influenced the nucleotide composition of NASP genes. Further, we show that selection has acted to maintain a high frequency of functionally relevant acidic amino acids in the region that interrupts TPR2. Third, functional diversity reported among several well characterized NASP family members can be explained in terms of quickly evolving TPR1 and TPR4 motifs. Fourth, NASP fish specific paralogs have significantly diverged at the protein level with NASP2 acquiring a NNR domain.
\end{abstract}

Keywords: NASP, H3/H4 transport, Hif1, N1/N2, Molecular evolution, Chromatin, Phylogenetics, SHNi-TPR, Histone chaperone

\section{Background}

The fundamental repeating unit of eukaryotic chromatin is the nucleosome that wraps a 146 bp stretch of DNA around a histone octamer consisting of two of each of histone H2A, H2B, H3 and H4 [1]. The transport of histones from the cytoplasm to the nucleus and their subsequent assembly into nucleosomes is mediated by a diverse set of proteins including histone chaperones [2] that are

\footnotetext{
* Correspondence: jeffrey.fillingham@ryerson.ca

'Department of Chemistry and Biology, Ryerson University, 350 Victoria St., Toronto M5B 2K3, Canada

Full list of author information is available at the end of the article
}

classified into several families based on their binding specificities and sequence and structural similarities [2]. One group of histone $\mathrm{H} 3 / \mathrm{H} 4$ chaperones is the nuclear autoantigenic sperm protein (NASP) family also known as the N1/N2 family.

The founding member of the NASP family is Xenopus laevis $\mathrm{N} 1 / \mathrm{N} 2$, which is expressed in oocytes and specifically binds histones $\mathrm{H} 3 / \mathrm{H} 4$, providing a mechanism for the storage of the soluble histones required for DNA replication in the early embryo $[3,4]$. NASP is the mammalian homolog of N1/N2 and was first described in rabbit testes as a highly autoantigenic protein which shares greater 
than $50 \%$ similarity to $\mathrm{N} 1 / \mathrm{N} 2[5,6]$. In mammals, NASP predominantly exists as two alternatively spliced isoforms; one is considerably longer than the other and is expressed in embryonic tissues and testis (tNASP) whereas the smaller version which lacks a region of 339 amino acids is called the somatic NASP (sNASP) and is highly expressed in all dividing cells [7]. NASP expression is tightly cell cycle regulated and its over-expression causes delay in cell cycle progression at the G1/S border $[7,8]$. NASP expression is essential in mammals as its gene disruption results in early embryonic lethality in mice [9]. Previous studies have shown that human NASP co-purifies with replication dependent and independent histones H3.1 and H3.3 respectively $[10,11]$. In human cells, newly synthesized histones $\mathrm{H} 3.1 / \mathrm{H} 4$ are thought to successively pass through at least four distinct cytosolic complexes $[10,12]$. In this context, NASP has been shown to be involved in accepting the histones from heat shock proteins, in the Hat1-dependent acetylation of $\mathrm{H} 4$, and subsequently handing over these histones to another histone chaperone, anti-silencing factor 1 (Asf1) through a physical interaction that has been shown to exist in humans and Saccharomyces cerevisiae $[10,12,13]$.

NASP family proteins share conserved motifs, possessing four tetratricopeptide repeats (TPR) where the second TPR is typically interrupted by a large acidic domain [14]. The NASP structural organisation is conserved from fungi to mice forming the SHNi-TPR protein family that was named for three initially characterized members, Silencing in the middle of the centromere protein 3 (Sim3Schizosaccharomyces pombe), Hat1p-interacting factor-1 (Hif1, S.cerevisiae), and NASP-interrupted TPR repeats [14]. The TPR motifs are 34 amino acid long amphipathic helices that form a helix-turn-helix arrangement and are thought to provide a structural scaffold for mediating protein-protein interactions [15]. Different TPR motifs in human NASP show different binding affinities for either histone $\mathrm{H} 3 / \mathrm{H} 4$ or $\mathrm{H} 1$. For example, the acidic patch present within TPR2 is critical for H1-binding whereas TPR4 mediates the interaction with core $\mathrm{H} 3 / \mathrm{H} 4$ histones [16]. These studies suggest that NASP might be involved in multiple functions involving histone dynamics (for review see [17]).

In addition to mammals, NASP homologs have been detected in several eukaryotic models such as $S$. cerevisiae (Hif1), S. pombe (Sim3) and Caenorhabditis elegans (NASP-1) $[14,18,19]$. In addition to their conserved motif arrangement, these proteins share functional similarity in that they generally demonstrate histone $\mathrm{H} 3 / \mathrm{H} 4$ chaperone activity [17]. Despite this, some functional diversity does exist among NASP family proteins. For example, Hif1 physically interacts with Hat1 and Hat2 as part of the Hat 1 complex that functions in the acetylation of newly synthesized histone H4 [19,20], whereas Sim3 appears to specifically function in deposition of the centromeric $\mathrm{H} 3$ variant [14], and does not appear to physically interact with Hat1 [21,22]. In addition to this, a recent report suggests that Sim 3 also has a general role in chromatin maintenance and acts as an $\mathrm{H} 3 / \mathrm{H} 4$ chaperone with some overlapping functional characteristics with Asf1 [23]. In C. elegans, NASP-1 has been implicated in female development through its interactions with histone deacetylase and TRA-4 proteins [18]. Additionally, human NASP functions in the fine tuning of a soluble reservoir of histones $\mathrm{H} 3 / \mathrm{H} 4$ by handing over excess histone $\mathrm{H} 3$ and $\mathrm{H} 4$ to heat shock proteins (HSP90/HSC70) for chaperone mediated autophagy [24].

In humans, NASP expression is significantly altered in a variety of cancers including those of the ovary and prostate [25-27]. Despite the demonstrated role of NASP in a wide range of cellular processes, questions remain about the underlying mechanistic details of NASP function. Recently, we found that a NASP family protein, NASPrelated protein 1 (Nrp1) co-purifies with Asf1 in the ciliate protozoan Tetrahymena thermophila [28] suggesting that the Asf1-NASP physical interaction is evolutionarily conserved in eukaryotes. Molecular evolutionary analysis has the potential to provide useful insights into protein function as well as providing information about changes in interacting partners $[29,30]$. Molecular evolutionary analyses of the proteins involved in the transport of histones $\mathrm{H} 3 / \mathrm{H} 4$ including HSP90, Asf1 and Importin $\beta$ have previously been reported and suggest that these proteins are highly conserved throughout the eukaryotes [31-33]. Although NASP has been suggested to be equally widely present among eukaryotes [34], a comprehensive phylogenetic analysis of NASP family proteins is lacking.

We present here a comprehensive phylogenetic analysis of NASP family proteins. Our analysis indicates that NASP is conserved across all of the major eukaryotic lineages ranging from the excavata to the crown group (animals, fungi, amoebozoans and plants) suggesting that the NASP histone chaperone was most likely present in the LECA. Furthermore, we show that in addition to the conserved arrangement of the four TPR motifs, an overall negatively charged nature is preserved in NASP family members suggesting that diversification of these proteins during eukaryotic evolution must have been determined by strong functional and structural constraints. High acidic amino acid composition in the TPR2 interruption region has been maintained by the presence of strong selection which in turn has biased the nucleotide composition in the corresponding NASP gene. Furthermore, we show that among ray-finned fish, NASP has undergone gene duplication and two paralogs exist under different genomic $\mathrm{G}+\mathrm{C}$ environments which suggests functional diversification of the two proteins. The present work combined with previous evolutionary analyses 
on Asf1, HSP90 and Importin $\beta$ proteins, suggests that histones $\mathrm{H} 3 / \mathrm{H} 4$ are transported via an evolutionarily conserved pathway that most likely was present in the last eukaryotic ancestor.

\section{Results \\ NASP is highly conserved in eukaryotes}

We used PSI-BLAST searches with default parameters to identify putative NASP orthologs across a wide range of eukaryotic lineages including those of the crown group (animals, fungi, amoebozoans and plants), the Guillardia nucleomorph, chromalveolates (apicomplexans, ciliates, oomycetes, and diatoms), and excavatas (parabasalids and kinetoplastida). Searches were carried out using the nonredundant database available at the NCBI website (http:// www.ncbi.nlm.nih.gov/) and identified sequences were recovered in the reciprocal searches (for accession numbers see Additional file 1: Table S1). The NASP family proteins contain a Pfam domain (PF10516) called SHNi-TPR which is an interrupted form of the TPR motif [14]. In order to correctly identify putative NASP orthologs, all sequences we obtained were analysed using the Pfam database [35] to confirm the presence of SHNi-TPR (PF10516) (see Methods for details). We queried the eggNOG orthology database [36] using human NASP and T. thermophila Nrp1protein sequences as reference, and observed that NASP forms an orthology group (KOG4563) with 117 protein members from 90 different eukaryotic species including several that were identified through our PSIBLAST searches. However, several previously unreported putative NASP orthologs not present in the eggNOG database were also identified in this study through PSIBLAST searches.

The distinctive feature of NASP family proteins is the presence of a TPR motif which is interrupted by a large acidic tract [14], and also revealed by our comparative sequence alignments (see below). In order to gauge the broad distribution of NASP proteins among eukaryotes, we prepared a hidden Markov Model (HMM) profile of the alignment of this region of the sequences culled from PSI-BLAST searches and screened through UniProtKB using HMMsearch [37]. This resulted in the recovery of significant hits $\left(\mathrm{e} \leq 10^{-5}\right)$ throughout eukaryotes with the notable exception of diplomonads (Additional file 2 ). Recovered sequences were analysed for the motif organisation and presence of SHNi-TPR/PF10516. Thus NASP appears widely distributed across the eukaryotes and may have emerged very early during the eukaryotic diversification. In fact the identification of putative NASP proteins through BLAST searches in excavata lineages (e.g., Parabasalids (Trichomonas vaginalis) and kinetoplastids (Trypanosoma)) which are thought to be highly divergent eukaryotic lineages [38-40] suggest that NASP was likely present in the LECA. Despite our repeated searches, we were unable to identify any putative NASP orthologs in diplomonads suggesting loss of this gene in these lineages.

Synteny, or gene neighbourhood analysis, often provides a good indication of correct identification of orthologs within closely related species [41]. We analysed the synteny of NASP among sequenced tetrapods and some other vertebrates using Genomicus [42]. We observed that gene pairs CCDC17, GPBP1L1 (coiled-coil domain containing 17 and GC-rich promoter binding protein 1-like 1, respectively) and AKR1A1, PRDX1 (aldo-ketoreductase family 1, member $A 1$ and peroxiredoxin 1, respectively) are found on either side of the NASP gene. We were able to trace back this conserved gene neighbourhood to the coelacanth Latimeria chalumnae which is considered the closest living relative of tetrapods [43]. These results suggest that the NASP genomic organisation is conserved among tetrapods.

\section{Conserved TPR domain organisation}

NASP proteins are defined by the presence of four TPR motifs where the second TPR is typically interrupted by large acidic patches. In addition, the TPR4 in S. pombe has also been predicted to contain a small insertion of 5 residues within the linker region that connects the two helices [14]. Our multiple sequence alignment (MSA) analysis indicates that the TPR motif sequence and arrangement are well conserved with TPR 1 and TPR3/4 flanking the interrupted TPR2 (Additional file 3: Figure S1A-E). The second residue of each TPR repeat often has a side chain that is either negatively charged or amidated [14]. For kinetoplastid lineages, we observed that the region interrupting TPR2 is significantly smaller than the others including humans, fungi and ciliates, consisting of 16 amino acid residues that mostly are hydrophobic and not predominantly acidic (For TPR organisation see: Additional file 3: Figure S1E). These kinetoplastid NASP do however possess an overall net negative charge with several acidic residues located mostly at the Nterminus. Furthermore, our analysis also indicates that T. vaginalis putative NASP is highly divergent and the TPR2 interruption region consists of only 10 residues with at least half of them acidic. However, several acidic residues are also found dispersed throughout the protein, thus giving it an overall net negative charge (data not shown). Interestingly, MSA and Pfam domain analysis also revealed that among a few selected lineages NASP has gained additional domains. For example, in addition to the conserved four TPRs, the putative NASP in Trichoplax adhaerens (phylum: Placozoa) contains an N-terminal MADF domain (PF10545) whereas Phytophthora infestans and P. sojae (both oomycetes) have gained an N-terminal Longin domain (PF13774). Such isolated instances of domain gain likely represent lineage specific functional divergence. 
MSA analysis of NASP family TPRs from a wide range of eukaryotic lineages permitted us to identify highly conserved residues that have been kept almost unchanged throughout evolution. For example, we noted that in addition to conserved hydrophobic residues found in all four TPRs, the amino acids at positions 7 and 8 are also highly conserved (Additional file 3: Figure S1A-E). For TPR1, the amino acids at positions 12 (leucine) and 21 (valine) have also remained almost unchanged suggesting that these residues are functionally relevant. Similarly, residues at positions 12 (leucine), 15 (alanine), 21 (tryptophan) and 24 (leucine) in TPR2 as well as positions 9 (glutamic acid), 11 (serine), 28/30 (leucine/leucine or isoleucine) in TPR3 and positions 10 (alanine) and 31 (isoleucine or leucine) of TPR4 are highly conserved. In addition, selected positions contain amino acids with similar biochemical properties (see the MSA in Additional file 3 ). It is also worth noting that a previously reported small C-terminal basic patch that could function as a nuclear localization signal [17] is also conserved in all NASP family members studied here (data not shown). Nevertheless, we also observed some lineage specific variations at selected amino acid positions. For instance, among vertebrates, TPR 1 amino acid positions 6 and 9 invariably are glycine and glutamine respectively, whereas among insects these residues have been substituted with serine and lysine, respectively. Similarly, amino acid positions 9 and 6 (both lysine) in TPR2 and TPR3, respectively, are highly conserved among vertebrates whereas they are more variable among other lineages (see the MSA in Additional file 3 ). The wide distribution of NASP in eukaryotic supergroups as well as the conserved pattern of TPR motifs strongly suggests that these amino acid variations represent a case of functional divergence among different orthologous proteins. In accordance with Dunleavy et al. [14] we also detected small insertions in the TPR4 of certain fungal lineages including S. pombe, S. japonicus, Cryptococcus gattii, Candida albicans, Coccidioides immitis and Neurospora crassa. Furthermore, Albugo laibachii (a genus of oomycetes) and Thalassiosira pseudonana (marine centric diatom) have each also experienced an insertion of 38 residues in the TPR4 (Additional file 3: Figure S1-D). The functional importance of these insertions is unknown. They may however represent convergent changes that arose independently among different lineages.

\section{Phylogenetic analysis of NASP}

After preliminary alignment and phylogenetic analyses, we reduced the total number of sequences representing different eukaryotic super groups for further investigation. Our global sequence alignments (data not shown) indicate that the TPRs constitute the most conserved regions of NASP family proteins. We therefore used the conserved blocks of amino acids comprising TPRs1-4 to reconstruct the protein phylogeny. The phylogeny shown in Figure 1 was reconstructed using MSA of 52 putative NASP protein sequences identified across diverse eukaryotic lineages. In order to assess the robustness of our results, we combined Bayesian analysis and maximum likelihood methods under the rtREV + F substitution model with gamma distributions as predicted by Prottest version 3.2 [44] and MEGAv5.2.1 [45]. Trypanosoma cruzi and T. brucei were used as out groups since these organisms are believed to diverge earlier than any other organism represented on the tree (Figure 1) [46-49].

The resulting phylogenetic tree recovered different taxonomic groups with well-defined topology as indicated by high statistical support for each internal node (Figure 1). This highly structured tree topology closely resembles the eukaryotic phylogeny inferred from rRNA sequences $[46,49]$. Thus with a few notable exceptions (see below), the overall topology suggests that NASP evolution parallels eukaryotic evolution. Chromalveolates branched first forming a monophyletic group followed by the diversification of the crown group lineages. Significant diversification is observed among different chromalveolata lineages as evidenced from the average amino acid variations ( $p=0.721 \pm 0.019$ substitutions per site) along with the varying branch lengths and grouping patterns on the tree (Figure 1). Ciliates form their own subgroup which is closely related to the apicomplexans with a monophyletic origin whereas oomycetes and diatoms are more closely related to each other than to the ciliates or apicomplexans. The crown group lineages with the exception of choanoflagellates and amoebozoans form a monophyletic group with a dichotomous topology such that fungi and plants fall within one monophyletic group whereas the remaining animals fall within another group.

The NASP differentiation within fungal and plant lineages occurred at the same time as evidenced from the monophyly of these groups with a moderate statistical support (ML bootstrap support of 35, Bayesian posterior probability of 65; Figure 1). This pattern of divergence deviates from accepted eukaryotic species phylogeny in which plants and fungi acquire distinct monophyletic groups [46-49]. Figure 1 demonstrates that plant lineages cluster together with relatively short branch lengths suggesting that NASP is highly conserved within plants. This observation is further supported by estimating average evolutionary divergence values $(p=0.251 \pm 0.027$ substitutions per site) for plant lineages. Fungal species form their own monophyletic subgroup with significant diversity apparent within basidiomycota and most ascomycota lineages. Long branches and moderately high average of amino acid sequence variations $(p=0.696 \pm 0.019$ substitutions per site) among nine fungal lineages provide further support for the presence of considerable differentiation among these proteins. Interestingly, S. cerevisiae and another 


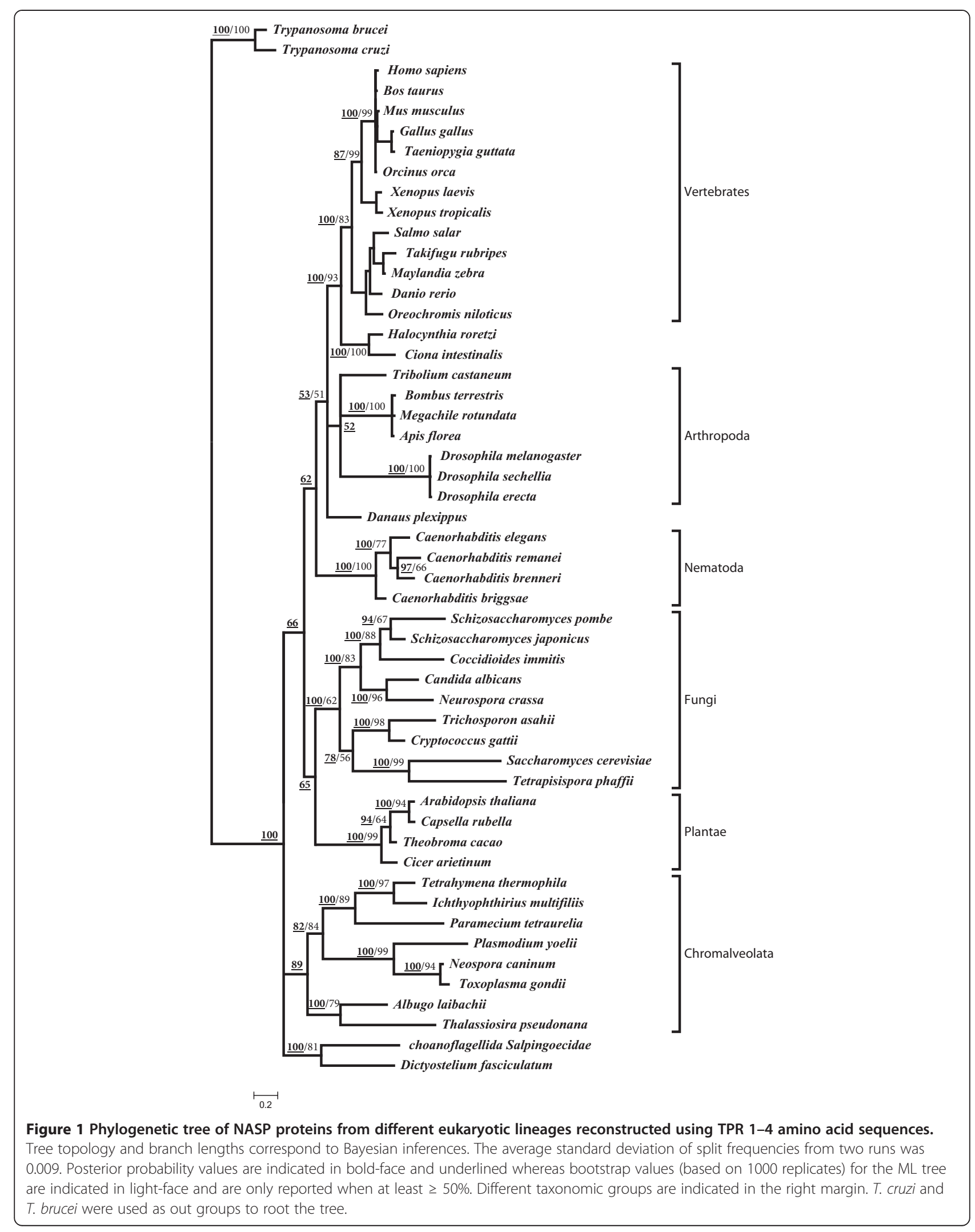


related ascomycota fungus Tetrapisispora phaffii [50] appear to be closely related to each other and quite divergent from the remaining ascomycotes as can be seen from their position in the phylogeny which is closer to basidiomycotes (Trichosporon asahii and C. gattii) than other ascomycota lineages. This suggests that Hif1 of $S$. cerevisiae and $T$. phaffii might have evolved faster in comparison to the other fungal lineages.

The chordate lineages form a monophyletic sub-group within which tunicates, which are thought to be the closest living relatives of vertebrates [51], group together and take the basal position followed by diversification among vertebrate lineages. Further analysis of the tree topology reveals that NASP lineages corresponding to fish cluster together whereas the differentiation of NASP in amphibians is followed by its diversification within mammals and aves. For NASP lineages corresponding to mammals and aves, we observed a minor disagreement between ML and Bayesian analyses. For the ML tree, aves lineages form a distinct subgroup as a sister clade to the mammals. Under Bayesian analysis however the differentiation of mammals and aves appears to have occurred concurrently. This discrepancy is consistent with the relatively low statistical support for the ML tree (Figure 1). As noted earlier, choanoflagellates and amoebozoans are the two exceptions to the general trend of NASP evolution seen for the crown group lineages. Both of these lineages take the basal position in the phylogeny before the differentiation of chromalveolates indicating that these proteins are highly diverged and may be subject to strict lineage-specific functional constraints. Consistent with this we observed a long stretch of serine/ threonine tandem repeats at the N-terminus of amoebozoan lineages (data not shown). Human NASP proteins have previously been predicted to contain a large number of potential serine/threonine phosphorylation sites with a few found towards the protein's $\mathrm{N}$-terminus [17]. However, the presence of serine/threonine tandem repeats at the $\mathrm{N}$-terminus may represent a lineage-specific adaptation although the functional significance remains unclear. An examination of multiple sequence alignments (Additional file 3: Figure S1, A-D) indicates amino acid variations at certain highly conserved and structurally relevant positions in TPRs1-4 for choanoflagellates. For example, glycine is invariably found at position 8 of TPR1 (Additional file 3). However in choanoflagellates, it has been substituted to the basic residue lysine. Furthermore, position 17 of TPR1 and position 4 of TPR2 in choanoflagellates have been substituted by lysine and glutamine respectively in place of conserved hydrophobic residues (Additional file 3: Figure S1-A,B). Similarly, we observed that position 9 of TPR3 which almost invariably contains an acidic amino acid (glutamic acid/ aspartic acid) has been replaced by a serine which is a polar neutral amino acid and only becomes acidic when phosphorylated, and highly conserved hydrophobic positions 21 and 13 have been substituted by glutamic acid and glutamine residues for TPR3 and TPR4, respectively (Additional file 3: Figure S1-C, D). Such variation at apparently key amino acid positions in the functional units (TPRs in this case) of a protein could possibly impact/ alter its function and/or interactions with other proteins.

To further examine evolutionary relationships among NASP proteins, we reconstructed a protein phylogeny based on complete protein sequences. The resulting phylogeny (Additional file 4: Figure S2) recovered the tree topology virtually identical to the one presented in Figure 1. The low statistical support for the tree, and slightly larger distances are indicative of an overall low similarity of the entire protein sequences (compare Table 1 with Additional file 1: Table S2). Nevertheless, this phylogeny based on complete protein sequences does provide two useful insights. First, the phylogenetic tree shown in Figure 1 is the most likely representation of NASP evolution with minor topological differences observed between the two phylogenies accounted for by the low statistical support for the phylogeny based on the entire protein sequence (Additional file 4: Figure S2; Figure 1). Second, NASP evolution is essentially dictated by the constraints present on TPR motifs.

TPR motifs provide a platform to mediate proteinprotein interactions and distinct NASP TPRs have been shown to have differential affinity for histones $\mathrm{H} 1$ and H3/H4 [15]. By estimating the divergence among different regions of the protein, specifically the TPRs (TPR1-4 combined in this case), the acidic region and the remaining $\mathrm{N}$ and C-termini (Additional file 1: Table S2), we observed significantly lower amino acid variation for the TPRs in comparison to the other regions, indicating that the TPRs constitute the most conserved regions in NASP proteins. Table 2 summarizes the overall amino acid variations (p-distances) for individual TPRs (TPR 1-4) across all the lineages without distinguishing among different taxonomic groups. From the estimated average amino acid $p$-distances, it appears that the TPR 1 and TPR 4 regions might be diverging more rapidly than TPR 2 and TPR3 (Table 2). This observation is further supported by calculating the amino acid $p$-distances for individual TPRs discriminating among different taxonomic groups which indicates that TPR1 and TPR4 generally have higher amino acid variations relative to TPR2 and TPR3 (Additional file 1: Table S3). In order to derive additional insight into the evolutionary processes that are shaping NASP TPRs, we re-constructed separate phylogenies for each TPR motif (Additional file 5: Figure S3, A-D). Difference in the clustering patterns among individual TPRs and/or divergence from the main protein phylogeny (Figure 1) has the potential to indicate 
Table 1 Average number of amino acid and nucleotide variations along with average synonymous $\left(p_{\mathrm{s}}\right)$ and non-synonymous $\left(p_{\mathrm{N}}\right)$ differences per site among NASP lineages from various taxonomic groups

\begin{tabular}{|c|c|c|c|c|c|c|}
\hline & $p_{\mathrm{AA}}(\mathrm{SE})$ & $p_{\mathrm{NT}}(\mathrm{SE})$ & $p_{\mathrm{S}}(\mathrm{SE})$ & $p_{\mathrm{N}}(\mathrm{SE})$ & $R^{a}$ & Z-test $^{\mathrm{b}}$ \\
\hline Vertebrate & $0.430 \pm 0.016$ & $0.353 \pm 0.008$ & $0.617 \pm 0.010$ & $0.263 \pm 0.011$ & 0.98 & $21.928^{* * *}$ \\
\hline Tunicata & $0.602 \pm 0.023$ & $0.417 \pm 0.012$ & $0.715 \pm 0.032$ & $0.323 \pm 0.019$ & 0.87 & $10.599^{* * *}$ \\
\hline Arthropoda & $0.561 \pm 0.012$ & $0.435 \pm 0.008$ & $0.611 \pm 0.012$ & $0.375 \pm 0.011$ & 1.06 & $11.673^{* * *}$ \\
\hline Nematoda & $0.451 \pm 0.020$ & $0.365 \pm 0.010$ & $0.676 \pm 0.020$ & $0.265 \pm 0.014$ & 1.05 & $16.227^{* * *}$ \\
\hline Fungi & $0.726 \pm 0.014$ & $0.578 \pm 0.008$ & $0.765 \pm 0.010$ & $0.522 \pm 0.011$ & 0.56 & $14.94^{* * *}$ \\
\hline Plants & $0.484 \pm 0.017$ & $0.400 \pm 0.013$ & $0.620 \pm 0.027$ & $0.327 \pm 0.017$ & 0.8 & $8.617^{* * *}$ \\
\hline Ciliates & $0.689 \pm 0.019$ & $0.465 \pm 0.011$ & $0.631 \pm 0.024$ & $0.426 \pm 0.015$ & 0.6 & $7.19^{* * *}$ \\
\hline Apicomplex & $0.531 \pm 0.020$ & $0.401 \pm 0.011$ & $0.655 \pm 0.021$ & $0.315 \pm 0.015$ & 0.9 & $12.447^{* * *}$ \\
\hline Euglenozoa & $0.423 \pm 0.024$ & $0.367 \pm 0.014$ & $0.703 \pm 0.030$ & $0.256 \pm 0.016$ & 0.87 & $12.856^{* * *}$ \\
\hline
\end{tabular}

$p_{\mathrm{AA}}, p_{\mathrm{NT}}, p_{\mathrm{S}}$ and $p_{\mathrm{N}}$, represent average number of amino acid, nucleotide, synonymous and non-synonymous nucleotide differences per site when calculated using the entire protein/nucleotide coding sequence along with the Z-test of selection. SE indicates standard error based on 1000 bootstrap replicates.

a average transition/transversion ratio.

${ }^{\mathrm{b}} \mathrm{H}_{1}: p_{N}<p_{S}$ and $\mathrm{H}_{\mathrm{o}}: p_{N}=p_{S}$.

${ }^{* * *} \mathrm{P}<0.001$.

how similar/dissimilar functional constraints might be present among different lineages for a given TPR. However when individual TPRs are studied in isolation, the reduction in total number of amino acid sites lowers the statistical support for the resulting trees, diminishing the significance of any useful inferences. Nevertheless, based on average $p$-distance values shown in Table 2 and Additional file 1: Table S3, it appears that TPR1 and TPR4 are the fastest evolving functional units of a given NASP ortholog and as such may account for the functional diversity observed among well characterized NASP family members.

\section{Constraints on NASP evolution}

We employed several complementary methods to estimate the selective forces operating on NASP evolution. We used the NASP nucleotide coding sequences from the representative lineages of all eukaryotic super groups (Additional file 6: figure S4-A). We carried out a codonbased Z-test of selection by comparing synonymous and non-synonymousvariations. Our results reveal extensive synonymous variation, considerably higher than non- synonymous variations $(* * * * \mathrm{P}<0.001)$ in all comparisons (Table 1). In fact the level of silent substitutions was generally very high when compared across all the species $\left(p_{\mathrm{S}}(\mathrm{SE})=0.73 \pm 0.006, p_{\mathrm{N}}(\mathrm{SE})=0.5 \pm 0.01 ;{ }^{* * *} \mathrm{P}<0.001\right.$; Additional file 6: Figure S4-A). These results suggest the presence of strong purifying selection acting at the protein level, presumably to maintain appropriate structure required for NASP function. To ascertain the functional constraints operating on different regions of the protein, we extended our analysis by distinguishing between TPRs (combined), the acidic region and the remaining parts of the protein for different taxonomic groups (Additional file 1: Table S2), as well as among individual TPRs (Table 2). The results indicate that the amount of silent variation is always significantly higher than the non-silent variation in all Z-test comparisons (for $P$-values and test statistics refer to Table 2 and Additional file 1: Table S2). Table 2 also reveals that although the number of synonymous variations is always higher than the non-synonymous, the non-synonymous variations are significantly greater for TPR $1 / 4$ in comparison to TPR $2 / 3$. These results support our observation that

Table 2 Average number of amino acid and nucleotide variations among different TPR domains

\begin{tabular}{|c|c|c|c|c|c|c|}
\hline & $p_{\mathrm{AA}}(\mathrm{SE})$ & $p_{\mathrm{NT}}(\mathrm{SE})$ & $p_{\mathrm{S}}(\mathrm{SE})$ & $p_{\mathrm{N}}(\mathrm{SE})$ & $R^{a}$ & Z-test ${ }^{b}$ \\
\hline TPR1 & $0.764 \pm 0.026$ & $0.599 \pm 0.015$ & $0.688 \pm 0.009$ & $0.568 \pm 0.022$ & $0.9^{\#}$ & $4.602^{* * *}$ \\
\hline TPR2 & $0.617 \pm 0.036$ & $0.499 \pm 0.021$ & $0.665 \pm 0.016$ & $0.437 \pm 0.027$ & $1^{\#}$ & $7.098^{* * *}$ \\
\hline TPR3 & $0.682 \pm 0.034$ & $0.530 \pm 0.022$ & $0.687 \pm 0.013$ & $0.476 \pm 0.031$ & $0.88^{\#}$ & $5.646^{* * *}$ \\
\hline TPR4 & $0.762 \pm 0.028$ & $0.582 \pm 0.016$ & $0.633 \pm 0.015$ & $0.564 \pm 0.023$ & $1.2^{\#}$ & $2.105^{* *}$ \\
\hline Entire Protein & $0.729 \pm 0.012$ & $0.562 \pm 0.008$ & $0.725 \pm 0.006$ & $0.510 \pm 0.011$ & 0.64 & $16.823^{* * *}$ \\
\hline
\end{tabular}

$p_{\mathrm{AA}}, p_{\mathrm{NT}}, p_{\mathrm{S}}$ and $p_{\mathrm{N}}$, represent the average number of amino acid, nucleotide, synonymous and non-synonymous nucleotide differences per site along with the Ztest of selection. SE indicates standard error based on 1000 bootstrap replicates.

average transition/transversion ratio.

\# calculated using Maximum composite likelihood method in this case.

${ }^{\mathrm{b}} \mathrm{H}_{1}: p_{N}<p_{S}$ and $\mathrm{H}_{\mathrm{o}}: p_{N}=p_{S}$.

${ }^{* * *} \mathrm{P}<0.001 ;{ }^{* *} \mathrm{P}<0.05$. 
TPR1/4 are diverging more quickly than the TPR2/3. In addition, overall nucleotide diversity for four TPRs calculated using a sliding window approach indicates that TPR1 and TPR4 exhibit higher amounts of divergence relative to TPR2/3 (Additional file 6: Figure S4-B).

Positive selection often affects only a few sites during a protein's molecular evolution. We therefore conducted an ML-based analysis for detecting the nature of selective forces operating upon each individual codon in NASP coding sequences among different taxonomic groups. We used the program HyPhy to conduct the analysis (see Methods for details) [52]. Our results do not indicate that any codons have been under positive selection at the statistical threshold of $\mathrm{P}<0.05$ (codon data not shown). These studies suggest that the purifying selection has been the dominant factor during NASP evolution.

We assessed the codon usage bias among different NASP lineages. It has been shown that different organisms exhibit preferences for the use of different synonymous codons [53]. The strength of codon bias has been shown to vary across genes within each genome and factors influencing codon bias may include selection for translational accuracy and efficiency, and GC bias [54-56]. We calculated the overall degree of codon bias for NASP genes among different taxonomic groups using the effective number of codons (ENC) [57]. The higher ENC value of 61 signifies that all synonymous codons are equally used and there is no bias in codon usage whereas a lower value of 20 indicates an extreme bias suggesting that only a preferred codon is used in each synonymous class. In the case of NASP we did not find any significant codon bias with the exception of ciliates (Table 3). The overall ENC values range from $49.89 \pm 5.82$ (for tunicates) to $54.64 \pm 3.43$ (for insects) whereas ciliates indicate a slightly biased trend with ENC of $40.73 \pm 2.37$ in comparison to the other chromalveolata lineages $\left({ }^{* *} P<0.05\right.$, t-test). Ciliates are known to have AT rich genomes and have previously been reported to exhibit a strong bias toward codons with low GC content [58,59] (Additional file 7: Figure S5). The observed lack of any significant codon usage bias among NASP genes is possibly due to the strong functional constraints at the protein level that allow for an extensive amount of silent divergence which in turn results in a decrease in codon usage bias. However, highly conserved and functionally relevant sites often exhibit a stronger bias in codon usage $[60,61]$. This possibility awaits further analysis for NASP among different lineages. Our analysis however of relative synonymous codon usage (RSCU) on human NASP suggests that highly conserved TPR residues isoleucine and arginine are preferentially encoded by AUU and AGA, respectively (for detailed view see: Additional file 7: Figure S6).

We investigated the role of natural selection for certain biased amino acids by determining the correlation
Table 3 Codon usage bias referred to as the effective number of codons (ENC) estimated in NASP discriminating different taxonomic groups

\begin{tabular}{ll}
\hline Taxonomic group & ENC \\
\hline Vertebrate & $50.69 \pm 1.41$ \\
Tunicate & $49.89 \pm 2.58$ \\
Arthropod & $54.64 \pm 3.43$ \\
Nematode & $51.13 \pm 2.59$ \\
Fungi & $50.98 \pm 5.82$ \\
Plant & $53.99 \pm 2.82$ \\
Ciliate & $40.73 \pm 2.37$ \\
Apicomplexa & $53.18 \pm 5.97$ \\
Euglenozoa & $53.78 \pm 2.63$ \\
\hline
\end{tabular}

coefficients between the genomic GC content and the frequency of GC-rich (GAPW) and GC poor (FYMINK) amino acids. Under the neutral model of evolution, GC rich and GC poor residues should positively and negatively correlate with the genomic GC content, respectively [62]. However, there will be no correlation if selection has influenced their frequency. Our results indicate the absence of any significant correlation between the genomic $\mathrm{GC}$ content and the frequency of $\mathrm{GC}$-rich residues and GC-poor residues (Figure 2; Table 4). Alanine (GC rich) and lysine (GC poor) represent the most abundant residues in each class. We do not observe significant correlation between the genomic GC content and the most represented amino acids in each class (alanine and lysine) (Figure 2, Table 4). These results are not consistent with the neutral model expectations and suggest a role for natural selection to maintain high frequencies of certain amino acids.

To further assess the role of natural selection, we compared the changes at the first codon position (nonsynonymous) with those at the third codon position (synonymous) in the most abundant residues. The nucleotide frequencies at these two codon positions should not be significantly different from each other according to the expectations of the neutral model [62]. Codons for the $\mathrm{GC}$-rich residue alanine possess $\mathrm{G}$ at the first position whereas codons for the GC-poor residue lysine have $A$ at the first position. Our analysis indicates that the mean $G$ $+\mathrm{A}$ content at the first position $(69.7 \pm 3.8)$ is significantly greater than the mean $\mathrm{G}+\mathrm{A}$ content $(46.54 \pm 15.8)$ at the four fold degenerate sites $(t$ test $=8.98, p=0.000$ ). Furthermore, the mean $\mathrm{G}+\mathrm{A}$ content at the first codon position and at four-fold degenerate sites do not significantly correlate $\left(r_{\mathrm{S}}=0.01, p\right.$-value $\left.=0.945\right)$. These results suggest that selection has influenced the nucleotide composition to maintain a high frequency of alanine and lysine.

Glutamic acid and aspartic acid are also found from high to moderate frequencies as determined by the amino 


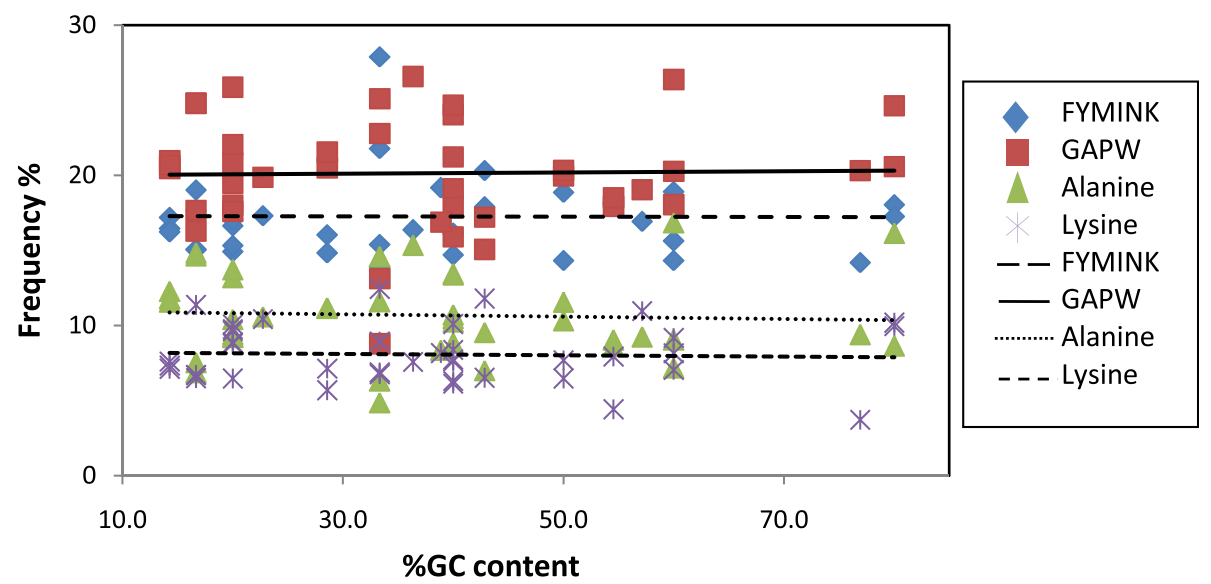

Figure 2 Relationship between the genomic GC content and GC-rich (GAPW) and GC-poor (FYMINK) residues. The relationship between most represented residues in each class (Alanine and Lysine) and GC content is also shown.

acid composition of NASP family proteins (data not shown; Additional file 1: Table S4). Codons for both of these residues have $G$ at their first position as do alanine codons. It is therefore reasonable to assume that the elevated levels of $\mathrm{G}+\mathrm{A}$ at the first codon position might be due to the selection for glutamic and aspartic acids in addition to alanine and lysine. This conclusion is further supported by the observation that nucleotide differences between the first and the third codon positions for alanine and lysine was most evident in the acidic domain that interrupts the TPR2 of the NASP proteins. The mean G+A content at the first position $(81.0 \pm 7.6)$ is significantly greater than the mean $\mathrm{G}+\mathrm{A}$ content $(6.7 \pm 23.1)$ at the four fold degenerate sites $(t$ test $=20.05, p=0.000)$. Furthermore, Z-test of selection also indicates the presence of strong purifying selection on the acidic domain of NASP proteins (Additional file 1: Table S2).

Provided that the clustering pattern of negatively charged NASP proteins is well differentiated with respect to different taxonomic groups (Figure 1), we assessed the potential role in selection of electrostatic interaction properties of different NASP proteins from each eukaryotic representative group (Additional file 7: Figure S7). Given that the crystal structure is not at present available for any of the NASP proteins, we used the I-TASSER server for structural predictions [63]. Subsequently, the predicted

Table 4 Genomic GC correlation with GC-rich and GCpoor amino acids in NASP family proteins

\begin{tabular}{lll}
\hline NASP family & $\begin{array}{l}\text { Spearman rank c } \\
\text { orrelation coefficient }\left(\boldsymbol{r}_{\boldsymbol{s}}\right)\end{array}$ & P-value \\
\hline Genomic GC vs. GAPW & -0.0896 & 0.899 \\
Genomic GC vs. FYMINL & 0.0634 & 0.968 \\
Genomic GC vs. Alanine & -0.188 & 0.749 \\
Genomic GC vs. Lysine & 0.0405 & 0.796 \\
\hline
\end{tabular}

structures were used to estimate the electrostatic potentials and their similarity indices using the web-PIPSA pipeline [64]. Our results indicate that differences in electrostatic potential is not the major evolutionary driving force as the representative NASP proteins from different taxonomic groups do not follow any particular clustering patterns (Additional file 7: Figure S7). These results suggest that selection has continuously acted in one direction to maintain high amounts of negatively charged amino acids particularly in the acidic domain, resulting in an overall net negative charge of NASP proteins presumably for their interaction with positively charged histones (also see Additional file 1: Table S4).

\section{NASP duplication}

In addition to the NASP splice variants present in most vertebrate species which generate functional diversity in NASP (for review see [17]), we observed that selected lineages have undergone gene duplication events. Gorilla, Felis and Taeniopygia among chordates and Caenorhabditis among nematodes each contain two NASP paralogs. Furthermore, we observed that among chordates, the ray-finned fish lineages also have undergone the gene duplication in the ancestral genome of clupeocephala ( 320 million years ago) [65], and Danio rerio has subsequently lost one copy of the gene. In most cases each of the two NASP paralogs has protein coding splice variants as assessed by 'ensembl' gene annotation [66]. This may generate additional functional diversity although the significance remains obscure.

To further assess the relationship among NASP paralogs, we reconstructed a phylogenetic tree using the nucleotide coding sequences from the above lineages by ML, maximum-parsimony (MP) and Bayesian methods (Figure 3). Our analysis indicates that NASP paralogs exhibit a polyphyletic origin and different NASP subtypes 


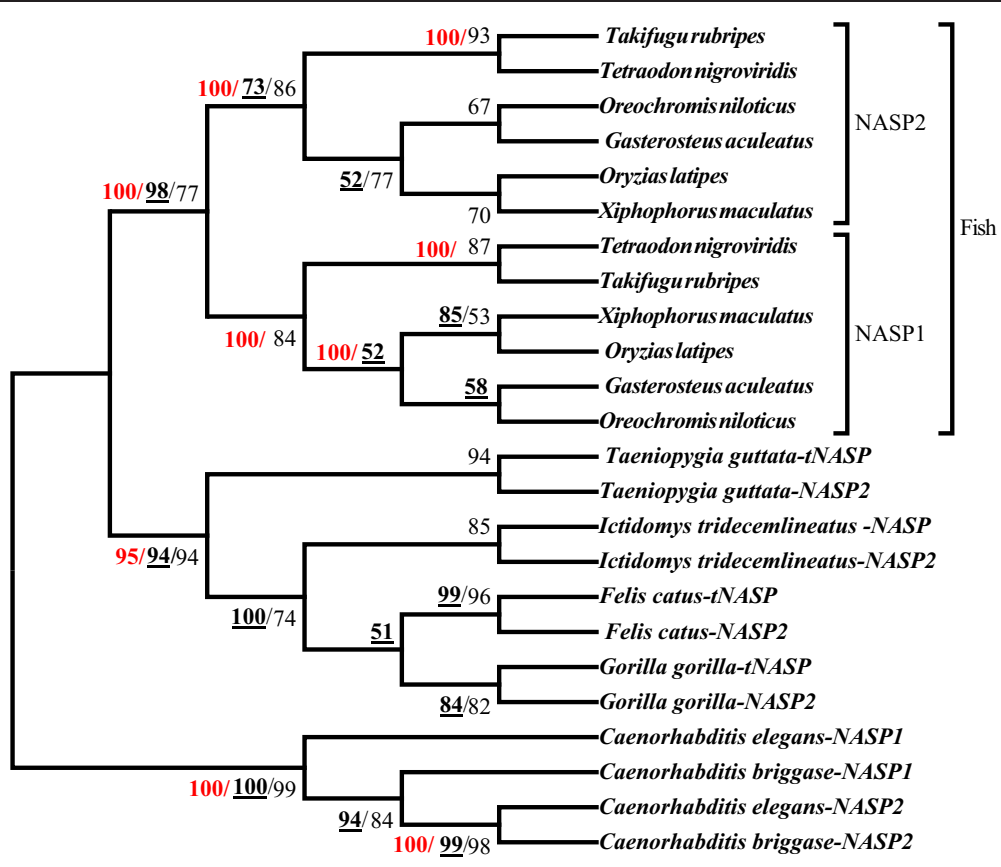

Figure 3 Phylogenetic relationship among different NASP paralogs inferred by ML, MP and Bayesian methods. The tree topology corresponds to ML estimations under the Tamura-Nei model. Branch lengths do not reflect genetic distance. Duplicated genes are referred to as NASP1 and NASP2 (see text for details). Confidence values for ML and MP trees are based on 1000 bootstrap replicates for each method and are indicated $(\geq 50 \%)$ in light-face and bold-face (underlined), respectively. Bayesian posterior probability values are indicated in red $(\geq 50 \%)$.

cluster based on the species to which they belong rather than the NASP subtypes (Figure 3). However, in the case of the fish lineages the clustering pattern is based on NASP subtypes rather than species indicating that the duplication event has only occurred once. Similarly, Caenorhabditis lineages also display a clustering pattern based on type rather than species suggesting that the gene duplication event occurred prior to the diversification of various Caenorhabditis species. Based on the phyletic patterns (Figure 3) and following the nomenclature for NASP paralogs already established for Caenorhabditis lineages, we designated the two paralogs as NASP1 and NASP2 for each of the lineage specific duplicated genes.

\section{Different genomic contexts for NASP1 and NASP2}

To gain insight into the genomic environment in which the two paralogs reside we analysed the synteny of duplicated genes. We observed that similar to other tetrapods, the ancestral NASP in the genome of clupeocephala has $C C D C 17$ and $A K R 1 A 1$ as its neighbours. Furthermore, among ray-finned fish lineages GPBP1L1 and PRDX1 genes which are also found in the NASP neighbourhood among other tetrapods are present as the immediate neighbours for at least one of the NASP paralogs (NASP1) whereas the direct neighbours of the NASP2 include GPC5 and $L R R C 7$ genes. Along with NASP, the TMEM69 gene which is found in the NASP neighbourhood among most of the vertebrates has also been duplicated suggesting a large segmental duplication event. This duplication ( 320MYA) may represent the signature of the whole genome duplication that took place in the ancestor of ray-finned fish [67]. Among other vertebrate lineage specific paralogs, NASP1 has the conserved gene neighbourhood (see above) whereas NASP2 neighbours include CHD7 in Gorilla, BICD1 in Felis catus and a gene that encodes a putative Zinc Finger protein in Taeniopygia guttata. Our analysis also indicates that the NASP1 immediate neighbour in C. elegans is $A P C-17$, whereas an uncharacterized gene lies next to the NASP2. From the analysis of gene structure and predicted protein sequences for T. guttata and F. catus paralogs, we observed that NASP2 are relatively smaller proteins (105 and 152 amino acids, respectively) and comprise only $\mathrm{N}$ - and C-termini respectively of the NASP1in these lineages. As these proteins lack TPR motifs or any other known functional units of NASP, we omitted them from further analyses.

Immediately after a hypothetical gene duplication event, purifying selection is expected to be relatively relaxed due to an initial functional redundancy, and to some extent it may permit the accumulation of divergence between the two paralogs [68-70]. To explain this in terms of protein function, two possible scenarios have been suggested: 1) in the case of sub-functionalization, the ancestral protein's function(s) are distributed between the duplicated genes; 


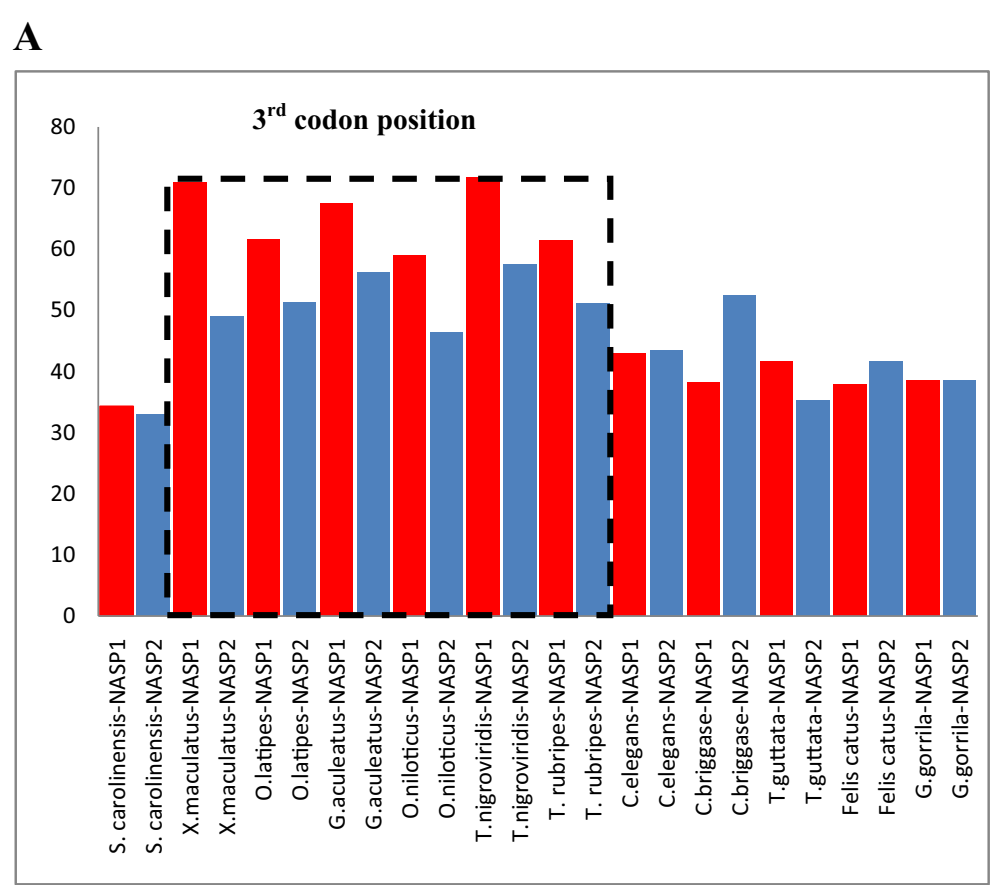

\section{B ENC Values}

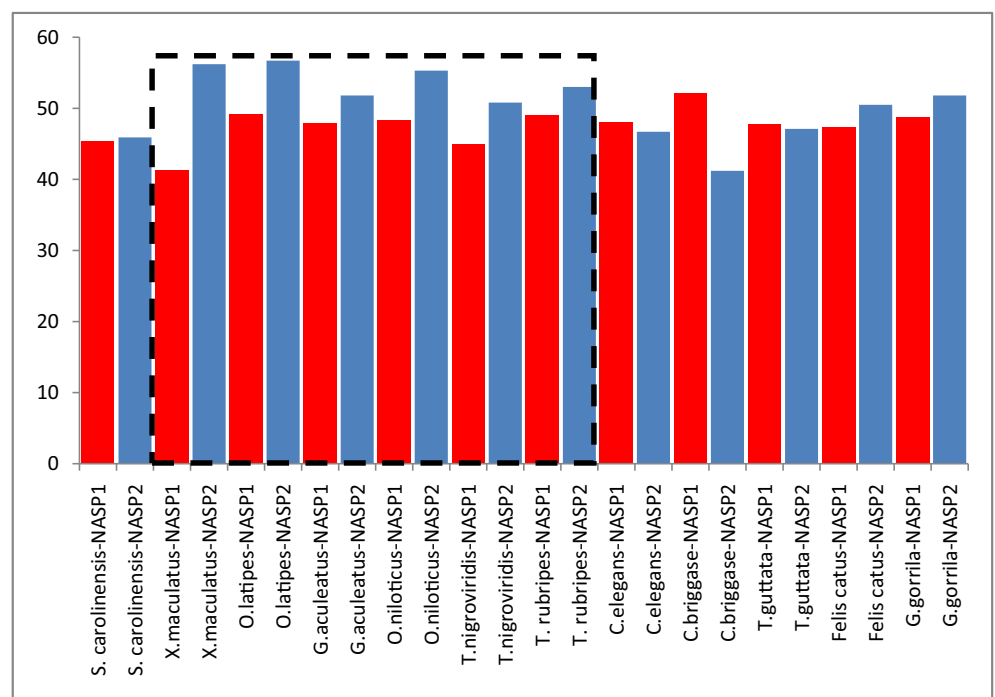

Figure 4 Comparison of \%GC3 and ENC values among NASP paralogs. A- GC content at the third codon position of NASP paralogs among different lineages. The box signifies estimated values for \%GC3 in fish lineages. B- The extent of codon usage bias referred to as ENC values among two NASP paralogs. The box indicates ENC for fish lineages.

2) in the case of neo-functionalization, the duplicated protein acquires a new function(s) [71,72]. To gain insight into possible functional divergence, we assessed genomic $\mathrm{G}+\mathrm{C}$ composition of the two paralogs. GC content has been correlated with various genomic properties, such as methylation patterns, recombination rates, gene density and gene expression patterns [73-76]. In addition, low GC content has also been associated with late replication [77] whereas highly expressed genes tend to be GC-rich [78].
In order to assess how evolutionary forces might have impacted on the genomic GC properties of the two paralogs, we calculated the GC content at the third codon position (GC3) because it has been shown to provide a good estimation of the GC content of the region in which the gene is located $[79,80]$. We observed a compositional bias in the GC content at the third codon positions for the fishes and Caenorhabditis whereas other lineage specific NASP paralogs did not show any significant differences in their 
\%GC3 (Figure 4A). We found that NASP1 always has a higher GC content than NASP2 among ray-finned fish whereas the opposite is true in the case of Caenorhabditis lineages. The most significant difference was noted for the fish Xiphophorus maculatus in which the two paralogs have $71 \%$ and $49 \%$ GC content at the third codon positions for $N A S P 1$ and $N A S P 2$, respectively (Figure 4A). Among fish lineages, NASP1 and NASP2 neighbouring genes GPBP1L1/PRDX1 and GPC5/LRRC7, respectively, also showed similar GC3 divergence. NASP1 neighbours (GPBP1L1/PRDX1) generally have a higher \% GC3 (overall average: 70\%/64\%, respectively) in comparison to the NASP2 neighbours (GPC5/LRRC7; overall average: 58\%/60\%, respectively) (Additional file 8: Figure S8 B-C). These observations suggest that the compositional bias among fish paralogs most likely arose after the duplication event due to their differential genomic locations. In contrast to the fishes the Caenorhabditis $N A S P 1 / 2$ neighbouring genes did not indicate any significant GC3 variations.

The single NASP in the distantly related $D$. rerio clusters with NASP2 on the phylogenetic tree (Additional file 8: Figure S8A), and has a 42.5\% GC3 (Additional file 7: Figure S5), suggesting that the lost copy of the gene is NASP1. However, the syntenic organisation of D. rerio $N A S P$ resembles that of $N A S P 1$ where it directly neighbours $C C D C 17$ and $A K R 1 A 1$ whereas GPBP1L1 is found next to $C C D C 17$. Low \%GC3 for $D$. rerio NASP correlates with the previous findings that the $D$. rerio genome has a lower GC content relative to the other fishes e.g., Oryzias latipes and Tetraodon [81].

The differences in the genomic environments may have a role in acquiring paralog-specific functions through distinct mutational biases [82] (for review, see [83]). High GC content has previously been correlated with elevated levels of gene transcription [84] as well as with the extent of codon usage bias [85]. We assessed the extent of codon bias between the two paralogs and observed that among fish lineages, NASP1 demonstrates more biased trends in codon usage than the NASP2 (Figure 4B). Similar trends were also seen for their corresponding neighbouring genes (Additional file 8: Figure S8B-C). Furthermore, it is also evident from Figure 4B that among Caenorhabditis, NASP2 exhibits slightly more biased codon usage than NASP1. Codon usage bias has previously been linked to selection for translational efficiency required for highly expressed genes and it has been shown that the preferred codons are often those which are recognised by the most frequent tRNAs [86]. For ray-finned fish and Caenorhabditis specific NASP paralogs however, compositional bias ( $\mathrm{G}+\mathrm{C}$ content) appears to be an important factor in shaping the codon usage. The observed compositional and codon usage bias could be linked to differential gene expression which has been proposed to provide indirect evidence for subfunctionalization and/or neo-functionalization [87].

\section{Functional differentiation of NASP Paralogs}

We examined the amino acid variations between the two paralogs in order to gain insights into functional differentiation. We observed that among fish lineages NASP1 has lost the N-terminal region consisting of the first 2530 amino acid residues that are found upstream of the start of TPR1. We also detected a small region within these $\mathrm{N}$-terminal residues that is highly conserved in NASP2 for all sequenced fish lineages (Figure 5). We refer to this conserved region as the NNR domain (NASP $\boldsymbol{N}$-terminal Region) and it demonstrates a characteristic pattern of S-X(5)-E-E-X-P-C-S-S-(S/T) where $\mathrm{X}$ is any amino acid. The significance of this domain is currently unclear. However it carries potential serine phosphorylation sites, as predicted by the KinasePhos 2.0 server [88], which may allow functionally important posttranslational modifications considering their conserved nature in fish NASP2 (Figure 5). The single retained copy of NASP in D. rerio also contains this NNR domain (Figure 5), further emphasizing our observations that the retained copy of the gene is NASP2 in this lineage.

We analysed the four TPR motifs and found that NASP paralogs in fish and Caenorhabditis have maintained the conserved arrangement of four TPRs whereas Gorilla NASP2 has lost the entire TPR1 and partially the TPR2 (see below). We focused on fish-specific NASP1/2 due to their clear pattern of evolutionary divergence as evident from Figure 3. We compared the two paralogous proteins with the reconstructed ancestral sequence and observed amino acid substitutions at key residues within each TPR suggesting that both lineages have diverged from the ancestral state (For MSA Additional file 8: Figure S8D-G). Our analysis indicates that TPR4 accumulated the greatest number of variations with 15 residues substituted followed by TPR1which has 6 residues changed. In order to assess the selective pressures operating on different regions of the two paralogs, we conducted a codon based Z-test of selection for each pair of sequences. While our analysis suggests the presence of purifying selection (data not shown and Additional file 1: Table S5), it should be noted that after a gene duplication, positive selection may affect only a few residues that might not be detected by pair-wise comparison methods [89]. Nevertheless amino acid variations in the functional units along with the loss of $\mathrm{N}$-terminal residues from NASP1 and the presence of an NNR domain in NASP2 provides indirect evidence for the functional differentiation between the two paralogs.

In contrast to the fish lineages, Gorilla NASP2 has lost the entire TPR1 and part of TPR2, as noted above. 


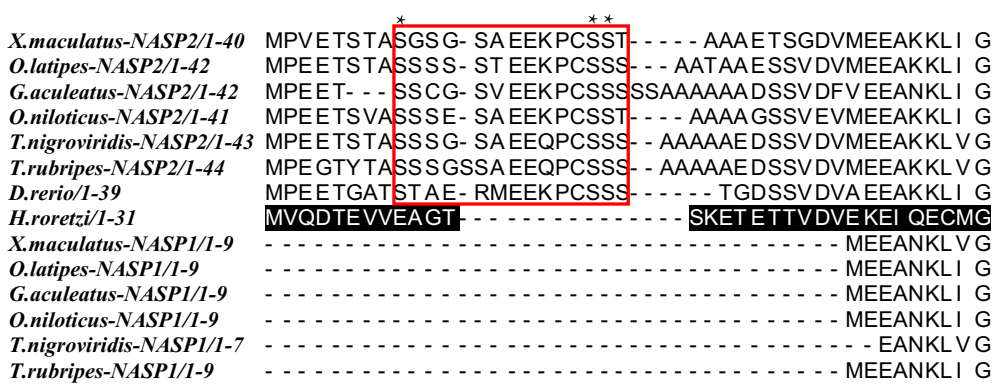

Figure 5 Multiple sequence alignments of the N-terminal region for NASP paralogs found in different fish lineages. The NNR region is highlighted in box whereas the out group lineage of Halocynthia roretzi is indicated in black. The asterisks (*) indicate the predicted conserved phosphorylation sites.

Furthermore, in the TPR2 interruption region it also lacks a stretch of 141 amino acid residues that are present in the NASP1. As a result NASP2 (582 amino acids in length) is much smaller than NASP1 (789 amino acids). Ensemble gene annotation indicates that unlike its other mammalian homologs (e.g. humans), Gorilla NASP1 does not have any splice variants equivalent in size to sNASP. These observations suggest that NASP2 might have assumed important functions regarding histone dynamics, e.g., similar to sNASP in other mammals. Our MSA analysis shows that the remaining regions of two paralogs including TPRs 3/4 are highly conserved with only a few amino acid substitutions (Additional file 8: Figure S8H-K). Low level of amino acid substitutions suggest that gene duplication probably occurred relatively recently. A Z-test of selection indicates that while most regions of the two proteins are evolving neutrally, the TPR2 interruption region demonstrates signs of positive selection (Additional file 1: Table S6). These results suggest that despite overall sequence conservation between Gorilla NASP1 and NASP2, the TPR2 interruption region has diverged significantly under the positive selection, possibly acquiring new function(s).

\section{NASP interacting partners are conserved}

NASP functions in the $\mathrm{H} 3 / \mathrm{H} 4$ transport pathway and interacts with a histone chaperone Asf1 as well as an Imp $\beta$ which is a karyopherin- $\beta$ family protein $[10,12,13]$. To estimate the wide distribution of NASP interacting proteins, we used Asf1 and Imp $\beta$ protein sequences from well characterized family members such as humans and S. cerevisiae and explored the non-redundant database with PSI-BLAST using default parameters. Reciprocally recovered sequences for both Asf1 and $\operatorname{Imp} \beta$ were used to prepare an HMMprofile for each of the proteins and Hmmsearch was conducted to scan the UniProKb. We recovered the putative Asf1 and Imp $\beta$ orthologs throughout the major eukaryotes, consistent with previous reports [31,33] (See Additional file 9 and Additional file 10 for accession numbers and species). The karyopherin- $\beta$ family proteins contain a Pfam domain IBN_N (PF03810) whereas the Asf1 family has a PF04729 domain. The recovered sequences from Hmmsearch were analysed for the presence of these domains in each case. The presence of Asf1 and $\operatorname{Imp} \beta$ proteins throughout the major eukaryotic groups suggests that similar to NASP, these proteins are also highly conserved and likely were present in the LECA.

\section{Discussion}

NASP is an essential protein in mammals and has a critical role in the trafficking of newly synthesized histones H3/H4 [10,12]. However, until now there has been a lack of information regarding the molecular evolution of NASP. We carried out a detailed phylogenetic analysis and establish that NASP evolution parallels that of eukaryotes. We also shed light on the selective forces operating on the evolution of NASP.

\section{Evolutionary conservation of NASP}

Our ability to detect NASP in a wide range of eukaryotic lineages including highly divergent $T$. vaginalis and an organism with a reduced genome (G. theta), argues that the protein is widely distributed throughout eukaryotes, and is a key generalized histone chaperone that most likely was present in the LECA. The histone chaperone Asf1 which has been shown to physically interact with NASP in humans, budding yeast and the ciliate protozoan $T$. thermophila $[10,13,28]$ is also highly conserved throughout the eukaryotes [32], and most likely was present in the LECA. We propose that that the NASPAsf1-Importin $\beta$ interaction is equally ancient and has a critical role in histone $\mathrm{H} 3 / \mathrm{H} 4$ metabolism.

Despite our extensive searches, we were unable to detect putative NASP homologs in diplomonads. This apparent loss is analogous to the absence of Histone Periodic Control 2 ( $\mathrm{Hpc} 2)$ in diplomonads [32]. $\mathrm{Hpc} 2$ is a subunit of an evolutionarily conserved $\mathrm{H} 3 / \mathrm{H} 4$ chaperone Histone Regulator (HIR) complex which physically interacts with Asf1 during replication independent chromatin assembly [90]. The HUN domain of Hpc2 has been suggested to be a histone tail-binding subunit of the HIR 
complex which might be critical for the transfer of $\mathrm{H} 3$ / H4 from Asf1 [32] and has been shown to be crucial for the HIR complex stability and preferential deposition of H3.3 [91].

Protein families are often categorized by the presence or absence of a conserved motif. In the case of NASP, the possession of interrupted forms of TPR (SHNi-TPR) motifs defines this family [14]. We have extended previous findings and shown that the structural arrangement of TPR motifs is conserved in NASP throughout the eukaryotic lineages. In this regard, NASP TPR analysis in kinetoplastids and parabasalids reveals that the TPR2 interruption is significantly smaller than the other family members and the acidic residues are either mostly $\mathrm{N}$ terminal or dispersed throughout the protein, respectively. Phylogenies based on rRNA sequences have previously shown that excavata are one of the earliest diverging eukaryotes $[46,49]$. It seems reasonable therefore to assume that the interruption of TPR2 with acidic patches occurred later during eukaryotic evolution, after the diversification of excavata lineages e.g., parabasalids and kinetoplastids.

A phylogeny based on the functional units of a protein may provide insights into a protein's functional diversification. The phylogeny shown in Figure 1 was reconstructed using conserved blocks of NASP TPR1-4 amino acid sequences, and indicates that NASP evolution largely parallels eukaryotic evolution. Existing genetic and biochemical evidence available for several well characterized NASP proteins including Hif1, Sim3, and sNASP reveal that in addition to being $\mathrm{H} 3 / \mathrm{H} 4$ chaperones, these proteins exhibit some level of functional diversity. For example, Hif1 has been shown to physically interact in a stoichiometric manner with Hat1 and Hat2 in S. cerevisiae and is involved with acetylation of newly synthesized histone $\mathrm{H} 4$, whereas Sim3 which is a fission yeast NASP homolog appears to function specifically in the deposition of centromere-specific histone H3 variant CENP-A [14,20]. Different NASP proteins cluster together based on their taxonomic groups suggesting a lineage-specific functionalization pattern. Such clustering patterns along with varying branch lengths observed among different groups provide a good indication for the possible functional diversity. For example, our analysis suggests that $S$. cerevisiae and $T$. phaffii might be evolving faster in comparison to the other fungal lineages and may participate in lineage specific functions. A test of positive selection using branch site models may help reveal the identity of any residues that might have been positively selected in these lineages. Our phylogenetic (Figure 1) and sequence alignment (Additional file 3) analyses indicate that NASP is highly conserved among chordates consistent with a previous report which indicated that NASP antibody has high cross reactivity across different chordate species [34]. The choanoflagellate and amoebozoan NASP proteins are however highly divergent in comparison to the other crown group lineages and several key residues in the TPR motifs that are conserved in other lineages have changed. These amino acid substitutions might be functionally significant although this hypothesis awaits biochemical and structural analysis.

\section{Selective constraints operating on NASP}

Estimation of amino acid $p$-distances suggests that TPR1 and TPR4 are evolving faster than TPR2 and TPR3 potentially accounting for the functional diversification observed among the NASP family members. This is further supported by our observation of more non-synonymous variations in TPR $1 / 4$ relative to TPR $2 / 3$ (Table 2 ). A deletion analysis of TPRs in a model system such as budding yeast [Fillingham et al. unpublished observations] that is amenable to a relatively straight forward biochemical analysis may provide further insights into specific TPR motif function. Comparison of synonymous and non-synonymous nucleotide substitutions indicated the presence of strong purifying selection that is operating at the protein level. This silent divergence indicates that NASP evolution has been subject to strong functional constraints maintaining essential structural features required for the protein's proper functioning. Similar functional constraints have also been reported for other histone interacting proteins such as the nucleoplasmin (NPM) family of $\mathrm{H} 2 \mathrm{~A} / \mathrm{H} 2 \mathrm{~B}$ histone chaperones [92]. Although the number of synonymous variations was always significantly higher than non-synonymous variations, by comparing the number of non-silent variations between the acidic and TPR regions, it is possible to assign significantly lower levels of non-silent variations in the latter. This indicates the presence of the strongest functional constraints on these regions operating in the form of purifying selection. Slightly higher levels of non-silent variations in the acidic regions could be due to the fact that selection for either aspartic acid or glutamic acid serves to maintain an overall negative charge.

Negatively charged residues found in NASP are important for the stability and proper functioning of the protein [16]. Selection has acted to maintain high frequency of these residues in the acidic region suggesting a deviation from neutrality. The neutral model of evolution predicts that the amino acid content of a protein is influenced by the nucleotide composition of its corresponding gene $[93,94]$. For example, the protein encoded by a gene with high GC bias will primarily be composed of amino acids that are encoded by GC-rich codons as a result of mutational bias. However, in contrast to the neutral model, the presence of strong selection at the protein level could alter the nucleotide composition bias. For NASP proteins, a deviation from neutrality is revealed when genomic GC content is compared with GC- 
rich and GC-poor residues. In contrast to the neutral model which predicts that GC-rich and GC-poor residues should have a positive and negative correlation with the genomic GC content, respectively, we observe no significant correlation between GC-rich/GC-poor residues and the genomic GC content. Comparison of the nucleotide frequencies at the first and third codon positions of lysine and alanine which comprise the most abundant residues in NASP provide insight into the role of natural selection. According to the neutral model, if the mutations are random and have equal probability of being fixed, then accordingly the four bases A, T, G, and C should occur with equal frequencies in the DNA $[62,93]$. Our results indicate that the frequency of $\mathrm{G}+\mathrm{A}$ is significantly higher at the first codon position in comparison to the third codon position. This difference is particularly evident when frequencies are compared for the acidic domains. The nucleotide frequencies have therefore been influenced by strong selection for lysine, alanine, glutamic acid, and aspartic acid. Most studies at the genome level do not support selection as the major determinant of the amino acid composition [95-97]. However, it has been suggested that local scale deviations from neutrality are generally overlooked in studies that aim to focus on genome-wide patterns [98]. Accordingly, a few studies have shown that natural selection is more important than mutational biases in shaping the nucleotide/amino acid composition [92,98-100]. For example, in proteo-bacteria, high levels of alanine and lysine in the Tol A protein are maintained due to strong selective pressures [98]. Similarly, for the NPM family of histone chaperones, high levels of adenine at the second codon position occur due to a selection for amino acids lysine and/or glutamic acid and aspartic acid [92]. The present work, in addition to other studies, provides useful insights regarding the role of natural selection in determining the nucleotide/ amino acid composition of a protein by demonstrating that elevated levels of G+A in NASP are maintained due to selection for alanine and/or glutamic acid/aspartic acid rather than the random mutational biases.

Several studies suggest that the unequal usage of synonymous codons exists because some codons are translated more efficiently and accurately and hence are the subject of selection (for review see [83]). We do not observe any significant codon bias among different NASP lineages (Table 3), although it has been shown that the sites that encode the most conserved and functionally important amino acids exhibit a greater bias in codon usage $[60,61]$ due either to selection or mutational bias. Further work will be required to investigate whether or not this is also the case for NASP proteins among different lineages. The determination of the most frequently used synonymous codons that encode the conserved residues in NASP TPRs may provide some insight. GC bias has previously been shown to positively correlate with the extent of codon usage bias [85]. In the case of ray-finned fish-specific NASP paralogs, we observed that NASP1 has higher GC content than NASP2 and accordingly shows more biased trends in codon usage. These differences may represent the possible functional divergence between the two paralogs. Furthermore, GC content has been linked with elevated gene expression levels $[84,86]$. The comparison of gene expression of the two paralogs should provide further insight into their functional differentiation. The amino acid substitutions in the TPRs as well as the absence of the N-terminal region in NASP1 and the presence of the NNR domain in NASP2 provide good indications that functional diversity exists between the two ray-finned fish specific paralogs. A test of positive selection using the branch-site method as well as determination of interacting partners and sub-cellular localization for each paralogous protein will further elucidate the existence of sub- or neo-functionalization.

\section{Conserved pathway of $\mathrm{H} 3 / \mathrm{H} 4$ transport}

Transport of histones $\mathrm{H} 3 / \mathrm{H} 4$ from the cytoplasm to the nucleus in yeast and human cells occurs in a stepwise fashion and is mediated by several proteins including heat-shock protein HSP90, NASP, Asf1 and Importin $\beta$ $[10,12]$. HSP90 and Asf1 have been shown to be highly conserved throughout the eukaryotes and most likely were present in the LECA [31,32,101]. A recent report established that the Karyopherin- $\beta$ family of proteins is also highly conserved throughout the eukaryotic lineages and was well established prior to the LECA [33]. In this regard, the present work completes the evolutionary analyses of the major proteins involved in the transport of $\mathrm{H} 3 / \mathrm{H} 4$ from the cytoplasm to the nucleus.

The physical interaction between HSP90-NASP, NASPAsf1 and Asf1-Importin $\beta$ has been well described in humans and yeast $[10,13]$. Our recent report established that Nrp1-Asf1-Importin $\beta$ physical interactions are wellconserved in a more divergent eukaryote $T$. thermophila [28]. The evidence for the physical interaction between heat-shock protein and NASP is still restricted to Opisthokonta lineages. Our preliminary proteomic analysis of Nrp1 in T. thermophila indicates that this interaction is conserved [Nabeel-Shah and Fillingham, unpublished observations]. The predicted functional annotation of $T$. thermophila Nrp1 using the COFACTOR server suggests that TPR1 residues constitute the potential heat shock binding sites (Additional file 11). This prediction is of interest because human NASP TPR2 and TPR3/4 have been demonstrated to be critical for histones $\mathrm{H} 1$ and H3/H4-binding, respectively [16]. Nevertheless, the validity and generality of this prediction requires experimental verification.

In order to re-capitulate and extend previous work on Asf1 and Karyopherin- $\beta$ family proteins we performed 
extensive database searches and identified putative Asf1 and Imp $\beta$ orthologs throughout the major eukaryotes. This, combined with previous studies [31,33] suggests that these proteins were most likely present in the LECA. These studies indicate a conserved transport pathway of histones $\mathrm{H} 3 / \mathrm{H} 4$ that most likely was present in the LECA and implicates HSP90, NASP, Asf1 and Importin $\beta$ proteins as the major components as their physical interactions have been described in divergent eukaryotic lineages including ciliates [28]. Other protein factors such as Hat1 complex, Codanin-1(human) and AIP1/AIP2 (ciliates) also play a role in histone $\mathrm{H} 3 / \mathrm{H} 4$ transport $[10,28,102]$. However the evolutionary origin of their role in the pathway is unclear at present and the available biochemical evidence is limited to either one or a small subset of taxonomic groups.

\section{Conclusions}

We have presented a detailed phylogenetic analysis of NASP family proteins. Several conclusions can be drawn from this study. First, NASP is widely distributed throughout the eukaryotes. It was most likely encoded in the genome of the last common ancestor of modern eukaryotes possibly representing an important innovation regarding the transport of $\mathrm{H} 3 / \mathrm{H} 4$ from the cytoplasm to the nucleus. Second, natural selection has influenced the frequency of nucleotides encoding NASP in order to maintain high frequency of functionally important glutamic and aspartic acid amino acids. Third, TPRs1/4 are diverging more quickly relative to TPR2 and TPR4 possibly accounting for the functional diversity that has been reported among well characterized family members. Fourth, NASP paralogs found among ray finned fish potentially represent separate functional entities with NASP2 acquiring an NNR domain.

\section{Methods}

\section{Sequence data retrieval and alignment}

Amino acid sequences for well characterized NASP family members including $X$. laevis $\mathrm{N} 1 / \mathrm{N} 2$ and human NASP were initially acquired from the NCBI protein database and were subsequently used as a query to search the nonredundant database with PSI-BLAST with default parameters. A phylogenomic approach was often used to increase the probability of identifying putative orthologs. For example, a putative NASP ortholog from the ciliate protozoan T. thermophila was used to detect further orthologs in other ciliate lineages. Sequences identified from each search were subsequently used as query for reciprocal recoveries. Protein sequences retrieved were analyzed at the Pfam database (http://pfam.sanger.ac.uk/) to examine the presence of SHNi-TPR (PF10516) [35]. However, SHNiTPR for highly divergent lineages such as ciliates was delineated through sequence alignments. HMMprofile was prepared using hmmbuild and the UniProtKB database was scanned using hmmsearch from the HMMER package [37]. Nucleotide coding sequences were acquired from GenBank with the exception of sequences for fungal lineages which were extracted from the Broad Institute of genome database (http://www.broadinstitute.org). Nucleotide coding sequences for tNASP lineages and duplicated genes were obtained from sequenced genomes available at Ensemble v72 [103].

Protein multiple sequence alignments were built using MUSCLE with default parameters [104] and Jalview [105] was subsequently employed to inspect and edit the alignments. TPR 1-4 regions were predicted using well characterized human NASP TPR sequences as a template and were aligned against consensus TPR sequences. Secondary structure prediction and further domain analysis was carried out using the Protein homology/analogy recognition engine V 2.0 (PHYRE 2) [106]. The alignment of nucleotide sequences was built on the basis of their translated amino acid sequences using MUSCLE as implemented in software MEGAv5.2.1 [45].

For Asf1 and Importin $\beta$, sequences for well characterized yeast and human proteins were retrieved from the NCBI database and were subsequently used as queries for BLAST searches with default settings to explore the non-redundant database. Retrieved sequences were aligned using MUSCLE and an HMMprofile was prepared as above. The UniProtKB database was scanned using HMMsearch limiting the search to eukaryotes only. For the karyopherin- $\beta$ family the retrieved sequences were analysed for the presence of IBN_N domain (PF03810) and sequences lacking the domain or smaller than 500 amino acids were eliminated from the final data set, in accordance with [33]. Similarly Asf1 significant hits were checked for the presence of Pfam domain PF04729.

\section{Synteny analysis and GC content calculation}

The Genomicus database v72.01 was queried using the human genome to investigate the genomic organisation of NASP family proteins [107]. DnaSP v5 was used to calculate GC content at the third codon positions [108].

\section{Molecular evolutionary and phylogenetic analyses}

Protein phylogenetic analyses were accomplished under maximum likelihood (ML) and Bayesian frameworks using NASP amino acid sequences for 52 different eukaryotic lineages. MEGA v5.2.1 (MEGA) was used for inferring an ML tree whereas Bayesian analysis was carried out using Mr. Bayes v3.2.0 [109]. We carried out 6 different protein phylogenetic analyses using data sets consisting of: 1) combined amino acid sequences for all four predicted TPRs (excluding the acidic domain) in each protein; 2) entire protein sequences; 3) four analyses based on amino acid sequences for each of four individual 
TPRs. The analysis based on all four combined TPR sequences was conducted under rtREV+G+F as selected by MEGA and ProtTest v3.2 [44]. For the remaining five analyses the model of protein evolution that best fits the data was selected using MEGA. For entire protein sequences, $\mathrm{rtREV}+\mathrm{G}+\mathrm{F}$ was used whereas for individual TPRs 1-4 model WAG+G was selected for TPR $1 / 2 / 4$ and rtREV+G for TPR 3. For individual TPR2 phylogenetic analysis we also included the acidic amino acid patches that interrupt the TPR2 acidic domain. For this, alignment positions with more than $50 \%$ gaps were eliminated using trimAL before conducting the analysis. For each data set, a total of 1000 bootstrap replicates were run to obtain statistical support for the resulting ML tree. For Bayesian inferences, for each data set, two runs of 2 million generations were conducted with 0.25 burn-in frequency and resulting posterior probabilities were taken as indicators of phylogenetic reliability. FigTree v1.4.0 (http://tree.bio.ed.ac.uk/software/figtree/) and MEGA were used to visualize the resulting phylogenies.

Phylogenetic trees based on nucleotide coding sequences were reconstructed using ML, Bayesian and MP method. A total of 1000 bootstrap replicates were run to obtain statistical support for the resulting MP and ML trees. ML analysis was carried out under the model $\mathrm{GTR}+\mathrm{G}+\mathrm{I}$ as selected by MEGA whereas MP trees were reconstructed using tree bisection and reconnection search method with search level 1 and with 10 replications for the random addition trees method. The ML phylogeny reconstruction to investigate the NASP paralogous relationships was conducted under the Tamura-Nei model with gamma distributions as selected by MEGA and statistical support was obtained based on 1000 replications. Bayesian analysis was conducted with two runs of 1 million generations $(0.25$ burn-in frequency) and resulting posterior probabilities were taken as indicators of phylogenetic reliability. For fish specific NASP paralogs, ancestral sequences corresponding to the pre-duplication node of the tree were inferred using MEGA under the empirical Bayesian method.

MEGA was used to analyse the nucleotide and amino acid compositions. To estimate the degree of amino acid and nucleotide divergence we used uncorrected differences ( $p$ distance) unless mentioned. This method was used because it gives better results than more complicated methods in particular for distantly related taxa due to its smaller variance. The modified Nei and Gojobori method [110] was used to compute the number of synonymous $(p s)$ and non-synonymous $\left(p_{N}\right)$ nucleotide differences per site by providing transition/transversion ratios ( $\mathrm{R}$ ) in both cases. A complete deletion option was used to calculate the distances and the bootstrap method with 1000 replicates was employed for estimating the standard errors.

Several approaches were used to estimate the nature of selective forces operating on NASP evolution. A codon based Z-test of selection was carried out to study the presence and nature of selection. The alternative and null hypotheses were established as follows; $\mathrm{H}_{\mathbf{1}}: p_{N}<p_{S}$ and $\mathrm{H}_{\mathbf{o}}$ : $p_{N=} p_{S}$. Probability to reject the null hypothesis and Z-test statistics were obtained. We tested deviation from neutrality by determining if selection has influenced certain amino acids. The GC content at fourfold degenerate sites was assumed to represent the genomic GC content providing that the latter has previously been shown to be a good approximation of the former $[79,80]$. In addition, GC content at four-fold degenerate sites was used as an approximation to the neutral expectation. Spearman rank correlation coefficient was used to compute the correlations and standard regression analysis was conducted for statistical significance. A comparison of nucleotide frequencies at the first codon position (always non-synonymous for the residues studied here) and at fourfold degenerate codon positions (always synonymous) was carried out in order to assess the influence of mutation and selection bias. Statistical significance of the results was also assessed by conducting student's $t$ test. In addition, estimation of the positive selection operating on individual codons was conducted using an ML-based method. We used the HyPhy program which is implemented in MEGA5. It involves the ML reconstructions of ancestral states under a Muse-Gaut model [111] of codon substitution. A nucleotide substitution model 'GTR' was also used as selected by MEGA and a user defined tree topology was provided. For detecting positively selected codons, the test statistic of $\mathrm{dN}-\mathrm{dS}$ was used, where $\mathrm{dS}$ is the number of synonymous substitutions per site and $\mathrm{dN}$ is the number of nonsynonymous substitutions per site. Results were considered statistically significant for positive selection if the probability value was less than 0.05. Furthermore, the overall nucleotide variations (average number of synonymous and non-synonymous diversity per site) within TPRs 1-4 was estimated using a slide window approach with a window length of $50 \mathrm{bp}$ and step size of $10 \mathrm{bp}$. The estimation of codon usage bias among NASP genes was conducted as the effective number of codons (ENC). Both analyses were carried out using the program DnaSP v5. In addition, relative synonymous codon usage (RSCU) [112] was estimated by MEGA using nucleotide coding sequences of the human NASP gene. To this end, an RSCU value greater than 1 indicates that a particular codon is used more frequently than expected whereas a value less than 1 indicates the reverse. Accordingly, an RSCU value of 1 indicates no codon bias [106].

\section{Structural bioinformatics}

Tertiary structures for different NASP proteins were predicted using the I TASSER server (http://zhanglab. ccmb.med.umich.edu/I-TASSER/) [113] and subsequently were submitted to the web-PIPSA pipeline for 
estimating the electrostatic potentials. See Additional file 12 for details.

\section{Availability of supporting data}

The data set supporting the results of this article is available in the TreeBase repository: http://purl.org/phylo/ treebase/phylows/study/TB2:S15931 [114]. Supporting data are also included as additional files.

\section{Additional files}

Additional file 1: Table S1. Accession number of the sequences along with species name identified through PSI-BLAST. Table S2. Average number of amino acid and nucleotide variations among different regions of the NASP proteins discriminating various taxonomic groups. pAA, pNT, $p S$, and $p \mathrm{~N}$, represent the average number of amino acid, nucleotide, synonymous and non-synonymous nucleotide differences per site along with the Z-test of selection. SE indicates standard error based on 1000 bootstrap replicates. Table S3: Average number of amino acids among different TPRs discriminating various taxonomic groups. Table S4: Number of acidic residues $(D+E)$ and theoretical iso-electric points distinguishing between the entire protein and acidic regions for the proteins used in this study Table S5/6: Average number of amino $\mathrm{acid} /$ nucleotide variations and Z-test of selection among different regions of the NASP paralogs.

Additional file 2: List of proteins identified in the study as putative NASP through HMMsearch. Species names, accession numbers of UniProtKB and e-values are also provided.

Additional file 3: Figure S1. A-E:SHNi-TPR sequence alignment of predicted TPR1 to TPR4 (A-D, respectively), across diverse eukaryotic lineages. The hydrophobic residues in each TPR that define the motif are denoted in black background. In accordance with Dunleavy et al. [35], the second residue in each TPR and $9^{\text {th }}$ residue for TPR3 (C) are highlighted with red indicating that residues at these sites are generally acidic or amidated. Gaps in the TPR2 (B) indicate that it is an interrupted form of TPR. TPR 4 (D) for certain lineages contains insertions in the linker regions that connect two helices in a TPR. These insertions are not shown, however their position is highlighted by underlining the residues and representing them in white against the blue background. Conserved residues are coloured according to the clustalX colour coding system [115]. E- Structural comparison based on TPR domain architect among different NASP proteins.

Additional file 4: Figure S2. Phylogenetic tree of NASP proteins from different eukaryotic lineages reconstructed using entire protein sequences. Tree topology corresponds to bootstrap consensus ML tree reconstructed under the rtREV+G+F model. Branch lengths do not reflect genetic distance. Posterior probability values are indicated as underlined whereas bootstrap values based on 1000 replicates for the ML tree are indicated in light-face and are only reported when $\geq 50 \%$. For Bayesian analysis the average standard deviation of split frequencies from two runs was 0.0072. Different taxonomic groups are indicated in the right margin. Trypanosoma cruzi and Trypanosoma brucei were used as out groups to root the tree.

Additional file 5: Figure S3. A-D: Phylogenetic trees reconstructed using individual TPR motifs (TPR1-4 shown in A-D respectively) from different eukaryotic lineages. Tree topologies correspond to the bootstrap consensus ML trees and are shown in circular format. Branch lengths do not reflect genetic distance. Posterior probability values wherever possible are indicated as underlined whereas bootstrap values based on 1000 replicates for the $\mathrm{ML}$ tree are indicated in the light-face and are only reported when at least $\geq 50 \%$ (coloured clades). Trypanosoma cruzi and Trypanosoma brucei were used as out groups to root the trees in each case.

Additional file 6: Figure S4. A. Phylogenetic relationship among different NASP orthologs. Trees were reconstructed using nucleotide coding sequences with ML and MP methods. The tree topology corresponds to bootstrap consensus ML estimations under the GTR+G+l model. Branch lengths do not reflect genetic distance. Confidence values for ML and MP trees are based on 1000 bootstrap replicates and are indicated $(\geq 50 \%)$ in light-face and bold-face (underlined), respectively. Trypanosoma cruzi and Trypanosoma brucei were used as out groups to root the tree. B. Overall nucleotide diversity (Pi) among four TPRs corresponding to the lineages represented in Additional file 6: Figure S4A. A sliding window approach was used to calculate the overall synonymous/ non-synonymous differences per site.

Additional file 7: Figure S5. GC content at the third codon position of NASP orthologs in various eukaryotic lineages. Figure S6. Relative Synonymous Codon Usage (RSCU) values for human NASP calculated using the nucleotide coding sequences. Different amino acids and their corresponding codons are grouped in different colors. Figure S7. Electrostatic distances calculated from the similarity indices for the electrostatic potentials of various NASP family proteins shown in a color-coded matrix heat map. The distance between similarity indices (S) of every pair of molecules ( $a$ and $b$ ) were calculated using $D_{a, b}=\sqrt{2-2 S_{a, b}}$ [116]. The color code and the density plot are also provided. Red and orange colors indicate similar potentials whereas more distant electrostatic potentials are represented with blue colors. Overall distances range from 0.04472 to 1.22229 (maximum range is from 0 to 2 ). The tree along the side of the image assembles the proteins into groups with similar electrostatic potentials (epogram). Despite the overall negative charge, it is apparent from the epogram that various NASP family members are interspersed suggesting that differences in electrostatic potentials are not the major selective force during NASP evolution. This signifies that maintaining a net negative charge through selection for acidic residues (D/E) has the functional importance rather than lineage specific set electrostatic potentials.

Additional file 8: Figure S8. A. Phylogenetic relationship among different fish-specific NASP paralogs. Tree topology corresponds to the $\mathrm{ML}$ method under the JTT+G model as predicted by MEGA. Branch lengths do not reflect genetic distance. Alternatively, the same topology was also recovered by the neighbour joining method using p-distances. Confidence values for ML and NJ trees are based on 1000 bootstrap replicates and are indicated ( $\geq 50 \%)$ in light-face and boldface (underlined), respectively. The tree is rooted with Halocynthia roretzi and Ciona intestinalis. B-C: \%GC3 content and ENC values are shown in chart diagrams for the fish-specific NASP1 and NASP2 neighbouring genes, respectively. The black line separates GC3 and ENC whereas blue and red colors represent different neighbouring genes as indicated in the chart legends. D-G. Predicted fish-specific NASP1/2 TPRs 1-4 sequence alignments are shown in figures B to $E$, respectively. Reconstructed ancestral sequence using the ML method is shown in black background whereas different amino acid substitutions in each TPR motif for two paralogous proteins are shown in blue. H-K. Predicted NASP1/2 TPRs 1-4 sequence alignments are shown in figures $\mathrm{H}$ to $\mathrm{K}$, respectively, for various lineages.

Additional file 9: List of proteins identified in the study as putative Asf1 through a HMMsearch. Species names, accession numbers of UniProtKB and e-values are provided.

Additional file 10: List of proteins identified in the study as putative Imp $\beta$ through a HMMsearch. Species names, accession numbers of UniProtKB and e-values are provided.

Additional file 11: A- Nrp1 predicted structure displayed in a surface representation complex with HSP90 peptide. Functional annotation was carried out using the COFACTOR server (Predicted GO terms for molecular function and biological process are: GO: 0022892 and GO: 0006606, respectively). The interaction was predicted using the structure of the designed TPR module complex with HSP90 (PDB ID: 3KD7) as reported by Cortajarena et al. [117].The arrow indicates the position of the peptide. B- Detailed view of HSP90 peptide interaction with Nrp1 residues. HSP90 peptide is shown in grey whereas the predicted interacting residues are shown in stick representation. $\mathbf{C}$ - Predicted structural model of Nrp1 shown in ribbon representation. Predicted TPRs 1, 3 and 4 are highlighted in different colors whereas acidic residues interrupting TPR2 are indicated in CPK models. The position of a coiled-coiled domain (CCD) predicted by SMART is 150-189 and is not shown. The position of a second predicted CCD found towards the C-terminus is highlighted. D- Space-filling 
representation of Nrp1 structure indicating the degree of conservation as calculated by the program ConSurf [118] based on Nrp1 amino acid sequence aligned against 52 NASP proteins from different organisms using MUSCLE. The color key is provided.

Additional file 12: Supplementary methods for structural prediction and calculation of electrostatic interaction properties.

\section{Competing interests}

The authors declare that they have no competing interests.

\section{Authors' contributions}

SN-S retrieved the sequences, prepared sequence alignments, performed the molecular evolutionary analyses, conducted Bayesian phylogenetic analysis, and drafted the manuscript. KA performed ML and MP phylogenetic analyses, prepared the figures and conducted domain analysis using Pfam and PHYRE2. REP edited the manuscript and participated in design and co-ordination of the study and data analysis. JSF conceived the study, participated in its design and coordination, edited the manuscript and participated in data analysis. All authors have read and approved the final manuscript.

\section{Acknowledgements}

We thank Dr. Clifford Brunk (UCLA) and Dr. Brian Golding (McMaster University) for critically reading the manuscript and providing helpful feedback. We also thank Dr. Jyoti Garg (York University) for her helpfu discussions. We thank two anonymous reviewers for their helpful feedback. This work was supported by: NSERC Discovery Grants to J.S.F (grant number: 386646-2010) and R.E.P (grant number: 539509), Ryerson University Health Sciences Fund (J.S.F), and an operating grant from CIHR to R.E.P (grant number: MOP13347).

\section{Author details}

'Department of Chemistry and Biology, Ryerson University, 350 Victoria St., Toronto M5B 2K3, Canada. ${ }^{2}$ Department of Biology, York University, 4700 Keele St., Toronto M3J 1P3, Canada.

Received: 27 February 2014 Accepted: 12 June 2014

Published: 20 June 2014

\section{References}

1. Luger K, Mader AW, Richmond RK, Sargent DF, Richmond TJ: Crystal structure of the nucleosome core particle at $2.8 \mathrm{~A}$ resolution. Nature 1997, 389(6648):251-260.

2. De Koning L, Corpet A, Haber JE, Almouzni G: Histone chaperones: an escort network regulating histone traffic. Nat Struct Mol Biol 2007 14(11):997-1007.

3. Kleinschmidt JA, Fortkamp E, Krohne G, Zentgraf H, Franke WW: Co-existence of two different types of soluble histone complexes in nuclei of Xenopus laevis oocytes. J Biol Chem 1985, 260(2):1166-1176.

4. Dilworth SM, Black SJ, Laskey RA: Two complexes that contain histones are required for nucleosome assembly in vitro: role of nucleoplasmin and N1 in Xenopus egg extracts. Cell 1987, 51(6):1009-1018.

5. Welch JE, Zimmerman LJ, Joseph DR, O'Rand MG: Characterization of a sperm-specific nuclear autoantigenic protein. I. Complete sequence and homology with the Xenopus protein, N1/N2. Biol Reprod 1990, 43(4):559-568.

6. Batova I, O'Rand MG: Histone-binding domains in a human nuclear autoantigenic sperm protein. Biol Reprod 1996, 54(6):1238-1244.

7. Richardson RT, Batova IN, Widgren EE, Zheng LX, Whitfield M, Marzluff WF, O'Rand MG: Characterization of the histone $\mathrm{H} 1$-binding protein, NASP, as a cell cycle-regulated somatic protein. J Biol Chem 2000, 275(39):30378-30386.

8. Alekseev OM, Bencic DC, Richardson RT, Widgren EE, O'Rand MG: Overexpression of the Linker histone-binding protein tNASP affects progression through the cell cycle. J Biol Chem 2003, 278(10):8846-8852.

9. Richardson RT, Alekseev OM, Grossman G, Widgren EE, Thresher R, Wagner EJ, Sullivan KD, Marzluff WF, O'Rand MG: Nuclear autoantigenic sperm protein (NASP), a linker histone chaperone that is required for cell proliferation. J Biol Chem 2006, 281(30):21526-21534.

10. Campos El, Fillingham J, Li G, Zheng H, Voigt P, Kuo WH, Seepany H, Gao Z, Day LA, Greenblatt JF, et al: The program for processing newly synthesized histones H3.1 and H4. Nat Struct Mol Biol 2010, 17(11):1343-1351.
11. Tagami H, Ray-Gallet D, Almouzni G, Nakatani Y: Histone H3.1 and H3.3 complexes mediate nucleosome assembly pathways dependent or independent of DNA synthesis. Cell 2004, 116(1):51-61.

12. Alvarez F, Munoz F, Schilcher P, Imhof A, Almouzni G, Loyola A: Sequential establishment of marks on soluble histones H3 and H4. J Biol Chem 2011, 286(20):17714-17721.

13. Fillingham J, Recht J, Silva AC, Suter B, Emili A, Stagljar I, Krogan NJ, Allis CD, Keogh MC, Greenblatt JF: Chaperone control of the activity and specificity of the histone $\mathrm{H} 3$ acetyltransferase Rtt109. Mol Cell Biol 2008, 28(13):4342-4353.

14. Dunleavy EM, Pidoux AL, Monet M, Bonilla C, Richardson W, Hamilton GL, Ekwall K, McLaughlin PJ, Allshire RC: A NASP (N1/N2)-related protein, $\operatorname{Sim} 3$, binds CENP-A and is required for its deposition at fission yeast centromeres. Mol Cell 2007, 28(6):1029-1044.

15. D'Andrea LD, Regan L: TPR proteins: the versatile helix. Trends Biochem Sci 2003, 28(12):655-662

16. Wang H, Ge Z, Walsh ST, Parthun MR: The human histone chaperone sNASP interacts with linker and core histones through distinct mechanisms. Nucleic Acids Res 2012, 40(2):660-669.

17. Finn RM, Ellard K, Eirin-Lopez JM, Ausio J: Vertebrate nucleoplasmin and NASP: egg histone storage proteins with multiple chaperone activities. FASEB J 2012, 26(12):4788-4804

18. Grote P, Conradt B: The PLZF-like protein TRA-4 cooperates with the Gli-like transcription factor TRA-1 to promote female development in C. elegans. Dev Cell 2006, 11(4):561-573.

19. Poveda A, Pamblanco M, Tafrov S, Tordera V, Sternglanz R, Sendra R: Hif1 is a component of yeast histone acetyltransferase $B$, a complex mainly localized in the nucleus. J Biol Chem 2004, 279(16):16033-16043.

20. Ai $X$, Parthun MR: The nuclear Hat1p/Hat2p complex: a molecular link between type $B$ histone acetyltransferases and chromatin assembly. Mol Cell 2004, 14(2):195-205.

21. Tong K, Keller T, Hoffman CS, Annunziato AT: Schizosaccharomyces pombe Hat1 (Kat1) is associated with Mis16 and is required for telomeric silencing. Eukaryot Cell 2012, 11(9):1095-1103.

22. Kim HS, Mukhopadhyay R, Rothbart SB, Silva AC, Vanoosthuyse V, Radovani E, Kislinger T, Roguev A, Ryan CJ, Xu J: Identification of a BET family bromodomain/casein kinase II/TAF-containing complex as a regulator of mitotic condensin function. Cell Rep 2014, 6(5):892-905.

23. Tanae K, Horiuchi T, Yamakawa T, Matsuo Y, Kawamukai M: Sim3 shares some common roles with the histone chaperone Asf1 in fission yeast. FEBS Lett 2012, 586(23):4190-4196.

24. Cook AJ, Gurard-Levin ZA, Vassias I, Almouzni G: A specific function for the histone chaperone NASP to fine-tune a reservoir of soluble $\mathrm{H} 3-\mathrm{H} 4$ in the histone supply chain. Mol Cell 2011, 44(6):918-927.

25. Ali-Fehmi R, Chatterjee M, Ionan A, Levin NK, Arabi H, Bandyopadhyay S, Shah JP, Bryant CS, Hewitt SM, O'Rand MG, Alekseev OM, Morris R, Munkarah A, Abrams J, Tainsky MA: Analysis of the expression of human tumor antigens in ovarian cancer tissues. Cancer Biomark 2010, 6(1):33-48.

26. Alekseev OM, Richardson RT, Tsuruta JK, O'Rand MG: Depletion of the histone chaperone tNASP inhibits proliferation and induces apoptosis in prostate cancer PC-3 cells. Reprod Biol Endocrinol 2011, 9:50.

27. Ma W, Xie S, Ni M, Huang X, Hu S, Liu Q, Liu A, Zhang J, Zhang Y: MicroRNA29a inhibited epididymal epithelial cell proliferation by targeting nuclear autoantigenic sperm protein (NASP). J Biol Chem 2012, 287(13):10189-10199.

28. Garg J, Lambert JP, Karsou A, Marquez S, Nabeel-Shah S, Bertucci V, Retnasothie DV, Radovani E, Pawson T, Gingras AC, Gingras AC, Pearlman RE, Fillingham JS: Conserved Asf1-importin beta physical interaction in growth and sexual development in the ciliate Tetrahymena thermophila. J Proteome 2013, 94:311-326

29. Eisen JA: Phylogenomics: improving functional predictions for uncharacterized genes by evolutionary analysis. Genome Res 1998 8(3):163-167.

30. Altenhoff AM, Dessimoz C: Inferring orthology and paralogy. Methods $\mathrm{Mol}$ Biol 2012, 855:259-279.

31. Daganzo SM, Erzberger JP, Lam WM, Skordalakes E, Zhang R, Franco AA, Brill SJ, Adams PD, Berger JM, Kaufman PD: Structure and function of the conserved core of histone deposition protein Asf1. Curr Biol 2003, 13(24):2148-2158.

32. Balaji S, lyer LM, Aravind L: HPC2 and ubinuclein define a novel family of histone chaperones conserved throughout eukaryotes. Mol Biosyst 2009, 5(3):269-275. 
33. O'Reilly AJ, Dacks JB, Field MC: Evolution of the karyopherin-beta family of nucleocytoplasmic transport factors; ancient origins and continued specialization. PLOS ONE 2011, 6(4):e19308.

34. Finn RM, Browne K, Hodgson KC, Ausio J: sNASP, a histone H1-specific eukaryotic chaperone dimer that facilitates chromatin assembly. Biophys J 2008, 95(3):1314-1325.

35. Coggill P, Finn RD, Bateman A: Identifying protein domains with the Pfam database. Curr Protoc Bioinformatics 2008, Chapter 2:Unit 25.

36. Powell S, Szklarczyk D, Trachana K, Roth A, Kuhn M, Muller J, Arnold R, Rattei T, Letunic I, Doerks T, Jensen LJ, von Mering C, Bork P: eggNOG v3.0: orthologous groups covering 1133 organisms at 41 different taxonomic ranges. Nucleic Acids Res 2012, 40(Database issue):D284-D289.

37. Eddy SR: Profile hidden Markov models. Bioinformatics 1998, 14(9):755-763.

38. Hampl V, Hug L, Leigh JW, Dacks JB, Lang BF, Simpson AG, Roger AJ: Phylogenomic analyses support the monophyly of Excavata and resolve relationships among eukaryotic "supergroups". Proc Natl Acad Sci U S A 2009, 106(10):3859-3864

39. Rodriguez-Ezpeleta N, Brinkmann H, Burey SC, Roure B, Burger G, Loffelhardt W, Bohnert HJ, Philippe H, Lang BF: Monophyly of primary photosynthetic eukaryotes: green plants, red algae, and glaucophytes. Curr Biol 2005, 15(14):1325-1330.

40. Burki F, Shalchian-Tabrizi K, Minge M, Skjaeveland A, Nikolaev SI, Jakobsen KS, Pawlowski J: Phylogenomics reshuffles the eukaryotic supergroups. PLOS ONE 2007, 2(8):e790.

41. Kuzniar A, van Ham RC, Pongor S, Leunissen JA: The quest for orthologs: finding the corresponding gene across genomes. Trends Genet 2008 24(11):539-551.

42. Muffato M, Louis A, Poisnel CE, Roest Crollius H: Genomicus: a database and a browser to study gene synteny in modern and ancestral genomes. Bioinformatics 2010, 26(8):1119-1121.

43. Amemiya CT, Alfoldi J, Lee AP, Fan S, Philippe H, Maccallum I, Braasch I, Manousaki T, Schneider I, Rohner N, Organ C, Chalopin D, Smith JJ, Robinson M, Dorrington RA, Gerdol M, Aken B, Biscotti MA, Barucca M, Baurain D, Berlin AM, Blatch GL, Buonocore F, Burmester T, Campbell MS, Canapa A, Cannon JP, Christoffels A, De Moro G, Edkins AL: The African coelacanth genome provides insights into tetrapod evolution. Nature 2013, 496(7445):311-316

44. Darriba D, Taboada GL, Doallo R, Posada D: ProtTest 3: fast selection of best-fit models of protein evolution. Bioinformatics 2011, 27(8):1164-1165

45. Tamura K, Peterson D, Peterson N, Stecher G, Nei M, Kumar S: MEGA5: molecular evolutionary genetics analysis using maximum likelihood, evolutionary distance, and maximum parsimony methods. Mol Biol Evol 2011, 28(10):2731-2739.

46. Kumar S, Rzhetsky A: Evolutionary relationships of eukaryotic kingdoms. J Mol Evol 1996, 42(2):183-193.

47. Baldauf SL, Roger AJ, Wenk-Siefert I, Doolittle WF: A kingdom-level phylogeny of eukaryotes based on combined protein data. Science 2000, 290(5493):972-977.

48. Marande W, Lopez-Garcia P, Moreira D: Eukaryotic diversity and phylogeny using small- and large-subunit ribosomal RNA genes from environmental samples. Environ Microbiol 2009, 11(12):3179-3188.

49. Bolivar I, Fahrni JF, Smirnov A, Pawlowski J: SSU rRNA-based phylogenetic position of the genera Amoeba and Chaos (Lobosea, Gymnamoebia): the origin of gymnamoebae revisited. Mol Biol Evol 2001, 18(12):2306-2314.

50. Kurtzman CP: Phylogeny of the ascomycetous yeasts and the renaming of Pichia anomala to Wickerhamomyces anomalus. Antonie Van Leeuwenhoek 2011, 99(1):13-23.

51. Delsuc F, Brinkmann H, Chourrout D, Philippe $H$ : Tunicates and not cephalochordates are the closest living relatives of vertebrates. Nature 2006, 439(7079):965-968

52. Pond SL, Frost SD, Muse SV: HyPhy: hypothesis testing using phylogenies. Bioinformatics 2005, 21(5):676-679.

53. Bernardi G: The vertebrate genome: isochores and evolution. Mol Biol Evol 1993, 10(1):186-204.

54. Bulmer M: The selection-mutation-drift theory of synonymous codon usage. Genetics 1991, 129(3):897-907.

55. Gouy M, Gautier C: Codon usage in bacteria: correlation with gene expressivity. Nucleic Acids Res 1982, 10(22):7055-7074.

56. Akashi $\mathrm{H}$ : Codon bias evolution in Drosophila. Population genetics of mutation-selection drift. Gene 1997, 205(1-2):269-278.

57. Wright F: The 'effective number of codons' used in a gene. Gene 1990, $87(1): 23-29$
58. Martindale DW: Codon usage in Tetrahymena and other ciliates. J Protozool 1989, 36(1):29-34.

59. Salim HM, Ring KL, Cavalcanti AR: Patterns of codon usage in two ciliates that reassign the genetic code: tetrahymena thermophila and Paramecium tetraurelia. Protist 2008, 159(2):283-298.

60. Akashi H: Synonymous codon usage in Drosophila melanogaster: natural selection and translational accuracy. Genetics 1994, 136(3):927-935.

61. Stoletzki N, Eyre-Walker A: Synonymous codon usage in Escherichia coli: selection for translational accuracy. Mol Biol Evol 2007, 24(2):374-381.

62. Kimura M: Rare variant alleles in the light of the neutral theory. Mol Biol Evol 1983, 1(1):84-93.

63. Zhang Y: I-TASSER server for protein 3D structure prediction. BMC Bioinformatics 2008, 9:40.

64. Richter S, Wenzel A, Stein M, Gabdoulline RR, Wade RC: webPIPSA: a web server for the comparison of protein interaction properties. Nucleic Acids Res 2008, 36(Web Server issue):W276-W280.

65. Broughton RE, Betancur RR, Li C, Arratia G, Orti G: Multi-locus phylogenetic analysis reveals the pattern and tempo of bony fish evolution. PLoS Curr 2013, 5:ecurrents.tol.2ca8041495ffafd0c92756e75247483e.

66. Koscielny G, Le Texier V, Gopalakrishnan C, Kumanduri V, Riethoven JJ, Nardone F, Stanley E, Fallsehr C, Hofmann O, Kull M, Harrington E, Boué S, Eyras E, Plass M, Lopez F, Ritchie W, Moucadel V, Ara T, Pospisil H, Herrmann A, G Reich J, Guigó R, Bork P, Doeberitz Mv, Vilo J, Hide W, Apweiler R, Thanaraj TA, Gautheret D: ASTD: the alternative splicing and transcript diversity database. Genomics 2009, 93(3):213-220.

67. Meyer A, Van de Peer Y: From 2R to 3R: evidence for a fish-specific genome duplication (FSGD). BioEssays 2005, 27(9):937-945.

68. Lynch M, Conery JS: The evolutionary fate and consequences of duplicate genes. Science 2000, 290(5494):1151-1155.

69. Wagner A: Selection and gene duplication: a view from the genome. Genome Biol 2002, 3(5):reviews1012.

70. Zhang J: Evolution by gene duplication: an update. Trends Ecol Evol 2003 18(6):292-298

71. Conant GC, Wolfe KH: Turning a hobby into a job: how duplicated genes find new functions. Nat Rev Genet 2008, 9(12):938-950.

72. Innan H, Kondrashov F: The evolution of gene duplications: classifying and distinguishing between models. Nat Rev Genet 2010, 11(2):97-108.

73. Rao YS, Chai XW, Wang ZF, Nie QH, Zhang XQ: Impact of GC content on gene expression pattern in chicken. Genet Sel Evol 2013, 45:9.

74. Jabbari K, Bernardi G: CpG doublets, CpG islands and Alu repeats in long human DNA sequences from different isochore families. Gene 1998, 224(1-2):123-127.

75. Duret $L$, Mouchiroud D, Gautier C: Statistical analysis of vertebrate sequences reveals that long genes are scarce in GC-rich isochores. J Mol Evol 1995, 40(3):308-317.

76. Eisenbarth I, Vogel G, Krone W, Vogel W, Assum G: An isochore transition in the NF1 gene region coincides with a switch in the extent of linkage disequilibrium. Am J Hum Genet 2000, 67(4):873-880.

77. Woodfine K, Fiegler H, Beare DM, Collins JE, McCann OT, Young BD, Debernardi S, Mott R, Dunham I, Carter NP: Replication timing of the human genome. Hum Mol Genet 2004, 13(2):191-202.

78. Kudla G, Lipinski L, Caffin F, Helwak A, Zylicz M: High guanine and cytosine content increases mRNA levels in mammalian cells. PLoS Biol 2006, 4(6):e180.

79. Aota $\mathrm{S}$, Ikemura $\mathrm{T}$ : Diversity in $\mathrm{G}+\mathrm{C}$ content at the third position of codons in vertebrate genes and its cause. Nucleic Acids Res 1986 14(16):6345-6355.

80. Bernardi G: Isochores and the evolutionary genomics of vertebrates. Gene 2000, 241(1):3-17.

81. Han L, Zhao Z: Comparative analysis of $\mathrm{CpG}$ islands in four fish genomes. Comp Funct Genom 2008, 2008:565631-565637.

82. Abascal F, Corpet A, Gurard-Levin ZA, Juan D, Ochsenbein F, Rico D, Valencia A, Almouzni G: Subfunctionalization via adaptive evolution influenced by genomic context: the case of histone chaperones ASF1a and ASF1b. Mol Biol Evol 2013, 30(8):1853-1866.

83. Hershberg R, Petrov DA: Selection on codon bias. Annu Rev Genet 2008, 42:287-299

84. Vinogradov AE: Dualism of gene GC content and CpG pattern in regard to expression in the human genome: magnitude versus breadth. Trends Genet 2005, 21(12):639-643 
85. Hershberg R, Petrov DA: General rules for optimal codon choice. PLoS Genet 2009, 5(7):e1000556.

86. Kanaya S, Yamada Y, Kinouchi M, Kudo Y, Ikemura T: Codon usage and tRNA genes in eukaryotes: correlation of codon usage diversity with translation efficiency and with CG-dinucleotide usage as assessed by multivariate analysis. J Mol Evol 2001, 53(4-5):290-298.

87. Li WH, Yang J, Gu X: Expression divergence between duplicate genes. Trends Genet 2005, 21(11):602-607.

88. Wong YH, Lee TY, Liang HK, Huang CM, Wang TY, Yang YH, Chu CH, Huang HD, Ko MT, Hwang JK: KinasePhos 2.0: a web server for identifying protein kinase-specific phosphorylation sites based on sequences and coupling patterns. Nucleic Acids Res 2007, 35(Web Server issue):W588-W594.

89. Zhai W, Nielsen R, Goldman N, Yang Z: Looking for Darwin in genomic sequences-validity and success of statistical methods. Mol Biol Evol 2012, 29(10):2889-2893.

90. Tang Y, Poustovoitov MV, Zhao K, Garfinkel M, Canutescu A, Dunbrack R, Adams PD, Marmorstein R: Structure of a human ASF1a-HIRA complex and insights into specificity of histone chaperone complex assembly. Nat Struct Mol Biol 2006, 13(10):921-929.

91. Song Y, Seol JH, Yang JH, Kim HJ, Han JW, Youn HD, Cho EJ: Dissecting the roles of the histone chaperones reveals the evolutionary conserved mechanism of transcription-coupled deposition of H3.3. Nucleic Acids Res 2013, 41(10):5199-5209.

92. Eirin-Lopez JM, Frehlick $L$, Ausio J: Long-term evolution and functional diversification in the members of the nucleophosmin/nucleoplasmin family of nuclear chaperones. Genetics 2006, 173(4):1835-1850.

93. Sueoka N: Correlation between base composition of deoxyribonucleic acid and amino acid composition of protein. Proc Natl Acad Sci U S A 1961, 47(8):1141-1149.

94. Sueoka N: Directional mutation pressure and neutral molecular evolution. Proc Natl Acad Sci U S A 1988, 85(8):2653-2657.

95. Karlin S, Blaisdell BE, Bucher P: Quantile distributions of amino acid usage in protein classes. Protein Eng 1992, 5(8):729-738.

96. Foster PG, Jermiin LS, Hickey DA: Nucleotide composition bias affects amino acid content in proteins coded by animal mitochondria. $J \mathrm{Mol}$ Evol 1997, 44(3):282-288

97. Wang HC, Singer GA, Hickey DA: Mutational bias affects protein evolution in flowering plants. Mol Biol Evol 2004, 21(1):90-96.

98. Rooney AP: Selection for highly biased amino acid frequency in the TolA cell envelope protein of Proteobacteria. J Mol Evol 2003, 57(6):731-736.

99. Rooney AP, Zhang J, Nei M: An unusual form of purifying selection in a sperm protein. Mol Biol Evol 2000, 17(2):278-283.

100. Akashi H, Gojobori T: Metabolic efficiency and amino acid composition in the proteomes of Escherichia coli and Bacillus subtilis. Proc Natl Acad Sci U S A 2002, 99(6):3695-3700.

101. Chen B, Zhong D, Monteiro A: Comparative genomics and evolution of the HSP90 family of genes across all kingdoms of organisms. BMC Genomics 2006, 7:156.

102. Ask K, Jasencakova Z, Menard P, Feng Y, Almouzni G, Groth A: Codanin-1, mutated in the anaemic disease CDAl, regulates Asf1 function in Sphase histone supply. EMBO J 2012, 31(8):2013-2023.

103. Flicek P, Amode MR, Barrell D, Beal K, Brent S, Chen Y, Clapham P, Coates G, Fairley S, Fitzgerald S, Gordon L, Hendrix M, Hourlier T, Johnson N, Kähäri A, Keefe D, Keenan S, Kinsella R, Kokocinski F, Kulesha E, Larsson P, Longden I, McLaren W, Overduin B, Pritchard B, Riat HS, Rios D, Ritchie GR, Ruffier M, Schuster M: Ensembl 2011. Nucleic Acids Res 2011, 39(Database issue): D800-D806.

104. Edgar RC: MUSCLE: a multiple sequence alignment method with reduced time and space complexity. BMC Bioinformatics 2004, 5:113.

105. Waterhouse AM, Procter JB, Martin DM, Clamp M, Barton GJ: Jalview Version 2-a multiple sequence alignment editor and analysis workbench. Bioinformatics 2009, 25(9):1189-1191.

106. Kelley LA, Sternberg MJ: Protein structure prediction on the Web: a case study using the Phyre server. Nat Protoc 2009, 4(3):363-371.

107. Louis A, Muffato M, Roest Crollius H: Genomicus: five genome browsers for comparative genomics in eukaryota. Nucleic Acids Res 2013, 41(Database issue):D700-D705

108. Librado P, Rozas J: DnaSP v5: a software for comprehensive analysis of DNA polymorphism data. Bioinformatics 2009, 25(11):1451-1452.

109. Ronquist F, Teslenko M, van der Mark P, Ayres DL, Darling A, Hohna S, Larget B, Liu L, Suchard MA, Huelsenbeck JP: MrBayes 3.2: efficient
Bayesian phylogenetic inference and model choice across a large model space. Syst Biol 2012, 61(3):539-542

110. Zhang J, Rosenberg HF, Nei M: Positive Darwinian selection after gene duplication in primate ribonuclease genes. Proc Natl Acad Sci U S A 1998, 95(7):3708-3713.

111. Muse SV, Gaut BS: A likelihood approach for comparing synonymous and nonsynonymous nucleotide substitution rates, with application to the chloroplast genome. Mol Biol Evol 1994, 11(5):715-724.

112. Sharp PM, Tuohy TM, Mosurski KR: Codon usage in yeast: cluster analysis clearly differentiates highly and lowly expressed genes. Nucleic Acids Res 1986, 14(13):5125-5143.

113. Roy A, Kucukural A, Zhang Y: I-TASSER: a unified platform for automated protein structure and function prediction. Nat Protoc 2010, 5(4):725-738.

114. Nabeel-shah S, Ashraf K, Pearlman RE, Fillingham JS: Molecular evolution of NASP and conserved histone H3/H4 transport pathway. TreeBASE 2014. http://purl.org/phylo/treebase/phylows/study/TB2:S15931.

115. Larkin MA, Blackshields G, Brown NP, Chenna R, McGettigan PA, McWilliam $H$, Valentin F, Wallace IM, Wilm A, Lopez R, Thompson JD, Gibson TJ, Higgins DG: Clustal W and Clustal X version 2.0. Bioinformatics 2007, 23(21):2947-2948.

116. Wade RC, Gabdoulline RR, De Rienzo F: Protein interaction property similarity analysis. Intl J Quantum Chem 2001, 83:122-127.

117. Cortajarena AL, Wang J, Regan L: Crystal structure of a designed tetratricopeptide repeat module in complex with its peptide ligand. FEBS J 2010, 277(4):1058-1066.

118. Landau M, Mayrose I, Rosenberg Y, Glaser F, Martz E, Pupko T, Ben-Tal N: ConSurf 2005: the projection of evolutionary conservation scores of residues on protein structures. Nucleic Acids Res 2005, 33(Web Server issue):W299-W302.

doi:10.1186/1471-2148-14-139

Cite this article as: Nabeel-Shah et al:: Molecular evolution of NASP and conserved histone $\mathrm{H} 3 / \mathrm{H} 4$ transport pathway. BMC Evolutionary Biology 2014 14:139.

\section{Submit your next manuscript to BioMed Central and take full advantage of:}

- Convenient online submission

- Thorough peer review

- No space constraints or color figure charges

- Immediate publication on acceptance

- Inclusion in PubMed, CAS, Scopus and Google Scholar

- Research which is freely available for redistribution 


\section{Chapter 4: Discussion}

The work presented here in the form of a published article (Nabeel-Shah et al. 2014) suggests that NASP is a key generalized histone chaperone that most likely was present in the LECA. Interestingly, the histone chaperone Asf1 which has been shown to physically interact with NASP in humans, budding yeast and the ciliate protozoan T. thermophila has also been shown to be present in the LECA (Fillingham et al. 2008; Balaji et al. 2009; Campos et al. 2010; Alvarez et al. 2011). Together, these observations collectively suggest that NASP-Asf1 physical interaction is conserved throughout the eukaryotes with possibly important functions regarding chromatin metabolism.

Remarkably the gene network analyses of T. thermophila Nrp1, human NASP and budding yeast Hif1 indicated a conserved pattern of functional links (Fillingham et al. unpublished). For example, histones H3/H4, Asf1 (in humans Asf1a), and Hat1-Hat2 are found in the gene networks of all three NASP homologs. Similarly, mini-chromosome maintenance (MCM) complex (MCM2/3/5) that has a critical role in DNA replication during initiation and elongation phases (Lei 2005) is found in human and T. thermophila whereas DNA replication initiation factor CDC45 is found in the networks of all three lineages (Fillingham et al. unpublished). These studies suggest a possibility of conserved functions of NASP including DNA replication and $\mathrm{H} 3 / \mathrm{H} 4$ metabolism.

The main NASP phylogeny (Figure 1 in Nabeel-Shah et al. 2014) was reconstructed using conserved blocks of NASP TPR1-4 amino acid sequences, and indicates that NASP evolution largely parallels eukaryotic evolution. In fact, we found that different NASP proteins cluster together based on their taxonomic groups which suggest a lineage specific functionalization pattern. Such clustering patterns along with varying branch lengths observed among different groups provide a good indication for the possible functional 
diversity. For example, our analysis suggests that $S$. cerevisiae and $T$. phaffii (both Fungi of the Ascomycota lineage) might be evolving faster in comparison to the other fungal lineages and may participate in specie specific functions. To this end, test of positive selection using branch site models may help reveal the identity of any residues that might have been positively selected in these lineages.

\section{1- Selective constraints operating on NASP}

The TPR motifs constitute the functional units of NASP (Finn et al. 2012) and therefore we asked how selective forces might have impacted on them. We found that TPR1 and TPR2 have evolved relatively faster than TPR2 and TPR 3 potentially accounting for the functional diversification observed among the NASP family members. A deletion analysis of TPRs in a model system such as budding yeast (Dannah et al, in preparation) that is amenable to a relatively straight forward biochemical analysis may provide further insights into specific TPR motif function. Next we sought to examine the nature of selective forces operating on long term NASP evolution. We observed the presence of strong purifying selection that is operating at the protein level. This silent divergence indicates that NASP evolution has been subject to strong functional constraints aimed to maintain essential structural features required for the protein's proper functioning. Similar functional constraints have also been reported for other histone interacting proteins such as the nucleoplasmin (NPM) family of H2A/H2B histone chaperones (Eirín-López et al. 2006).

Negatively charged residues found in NASP are important for the stability and proper functioning of the protein (Wang et al. 2012). Our results indicate that selection has acted to maintain high frequency of these residues in the acidic region suggesting a deviation from neutrality. The neutral model of evolution predicts that the amino acid content of a protein is 
influenced by the nucleotide composition of its corresponding gene (Sueoka 1961; Sueoka 1988). For example, the protein encoded by a gene with high GC bias will primarily be composed of amino acids that are encoded by GC-rich codons as a result of mutational bias. However, in contrast to the neutral model, the presence of strong selection at the protein level could alter the nucleotide composition bias. For NASP proteins, a deviation from neutrality is revealed when genomic GC content is compared with GC-rich and GC-poor residues. In contrast to the neutral model which predicts that GC-rich and GC-poor residues should have a positive and negative correlation with the genomic GC content, respectively, we observe no significant correlations between GC-rich/GC-poor residues and the genomic GC content. In order to further assess the role of natural selection, we compared the nucleotide frequencies at the first and third codon positions of lysine and alanine which comprise the most abundant residues in NASP. According to the neutral model, if the mutations are random and have equal probability of being fixed, then accordingly the four bases A, T, G, and C should occur with equal frequencies in the DNA (Sueoka 1961; Sueoka 1988). Our results indicate that the frequency of $\mathrm{G}+\mathrm{A}$ is significantly higher at the first codon position in comparison to the third codon position. This difference is particularly evident when frequencies are compared for the acidic domains. These results indicate that the nucleotide frequencies have been influenced by strong selection for lysine, alanine, glutamic acid, and aspartic acid.

Most studies at the genome level do not support selection as the major determinant of the amino acid composition (Karlin et al. 1992; Foster et al. 1997; Wang et al. 2004). However, it has been suggested that local scale deviations from neutrality are generally overlooked in studies that aim to focus on genome-wide patterns (Rooney 2003). Accordingly, a few studies have shown that natural selection is more important than mutational biases in shaping the nucleotide/amino acid composition (Rooney et al. 2000; 
Akashi and Gojobori 2002; Eirín-López et al. 2006). For example, in proteo-bacteria, high levels of alanine and lysine in the Tol A protein are maintained due to strong selective pressures (Rooney 2003). Similarly, for the NPM eukaryotic family of histone chaperones, high levels of adenine at the second codon position occur due to a selection for amino acids lysine and/or glutamic acid and aspartic acidic (Eirín-López et al. 2006). The present work, in addition to other studies, provides useful insights regarding the role of natural selection in determining the nucleotide/amino acid composition of a protein by demonstrating that elevated levels of G+A in NASP are maintained due to selection for alanine and/or glutamic acid/aspartic acid rather than the random mutational biases.

\section{2- Conserved Pathway of H3/H4 Transport}

Transport of histones $\mathrm{H} 3 / \mathrm{H} 4$ from the cytoplasm to the nucleus in yeast and human cells occurs in a stepwise fashion and is mediated by several proteins including heat-shock protein HSP90, NASP, Asf1 and Importin $\beta$. HSP90 and Asf1 have been shown to be highly conserved throughout the eukaryotes and most likely were present in the LECA (Chen et al. 2006; Balaji et al. 2009). A recent report established that the Karyopherin- $\beta$ family of proteins is also highly conserved throughout the eukaryotic lineages and was well established prior to the LECA (O'Reilly et al. 2011). In this regard, the present work completes the evolutionary analyses of the major proteins involved in the transport of $\mathrm{H} 3 / \mathrm{H} 4$ from the cytoplasm to the nucleus.

The physical interaction between HSP90-NASP, NASP-Asf1 and Asf1-Importin $\beta$ has been well described in humans and yeast (Fillingham et al. 2008; Balaji et al. 2009; Campos et al. 2010; Alvarez et al. 2011). Our recent report established that Nrp1-Asf1 -Importin $\beta$ physical interactions are well-conserved in a more divergent eukaryote $T$. thermophila (Garg 
et al. 2013). In addition, the proteomic analysis of Nrp1 in T. thermophila presented in the part 1of this thesis indicates that this interaction is also conserved.

In order to re-capitulate and extend previous work on Asf1 and Karyopherin- $\beta$ family proteins we performed extensive database searches and identified putative Asfl and Imp $\beta$ orthologs throughout the major eukaryotes (Nabee-Shah et al. 2014). This, combined with previous studies suggests that these proteins were most likely present in the LECA. We suggest that histones $\mathrm{H} 3 / \mathrm{H} 4$ are transported via conserved pathway that most likely was present in the LECA and implicates HSPs, NASP, Asf1 and Importin $\beta$ proteins as the major components as their physical interactions have been described in divergent eukaryotic lineages including ciliates. Other protein factors such as Hat1 complex, Codanin-1(human) and AIP1/AIP2 (ciliates) also play a role in histone H3/H4 transport (Campos et al. 2010; Ask et al. 2012; Garg et al. 2013). However the evolutionary origin of their role in the pathway is unclear at present and the available biochemical evidence is limited to either one or a small subset of taxonomic groups. 


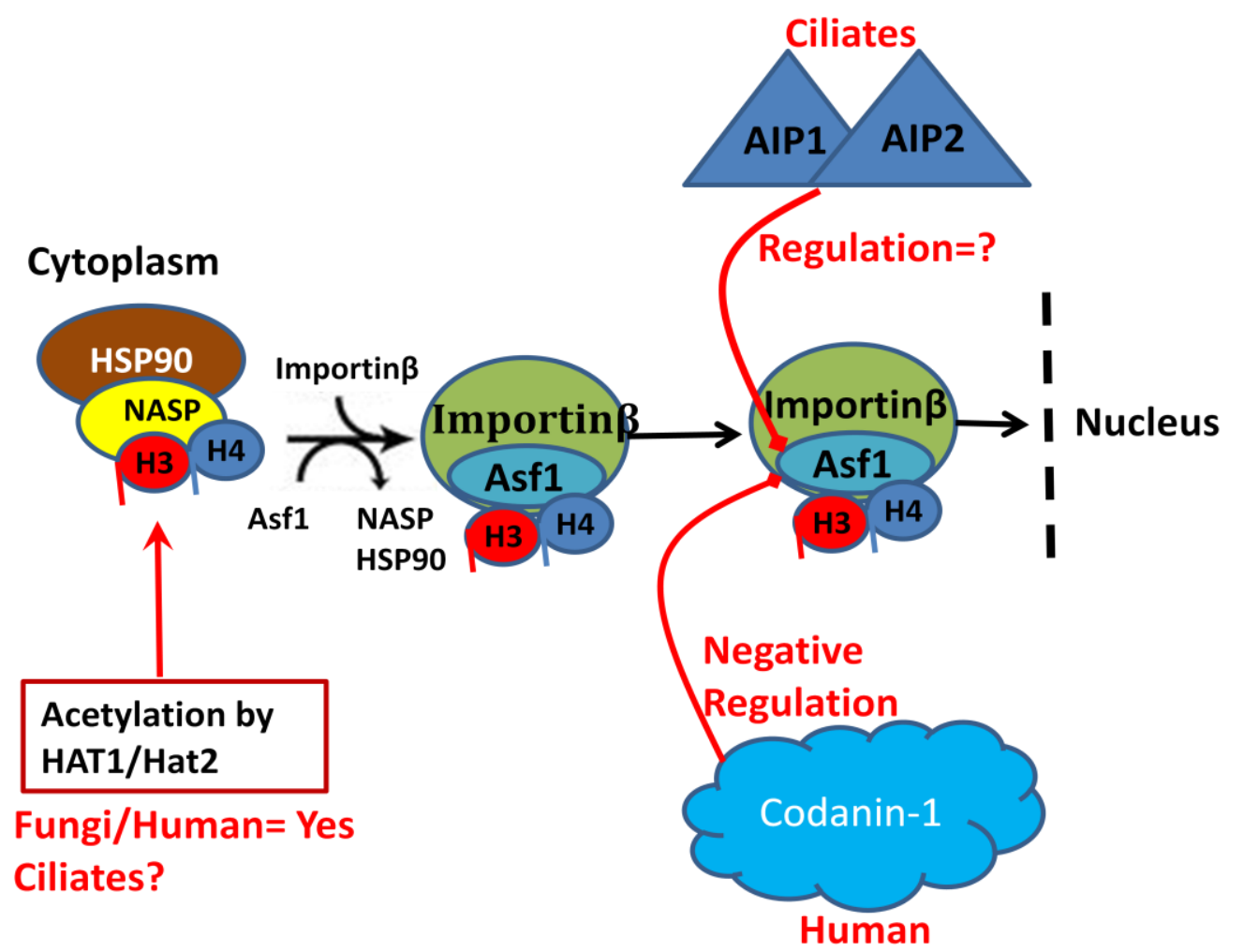

Hypothetical conserved pathway of $\mathrm{H} 3 / \mathrm{H} 4$ transport. The connections represented in red indicate that the interaction is either only limited to a small subset of lineages and/or its evolutionary origin is not clear thus far.

\section{3- Conclusions and future aspects}

We have presented a detailed phylogenetic analysis of NASP family proteins. Several conclusions can be drawn from this study. First, NASP was most likely encoded in the genome of the last common ancestor of modern eukaryotes possibly representing an important innovation regarding the transport of $\mathrm{H} 3 / \mathrm{H} 4$ from the cytoplasm to the nucleus. Second, natural selection has influenced the frequency of nucleotides encoding NASP in order to maintain high frequency of functionally important glutamic and aspartic acids. Third, TPRs1/4 are diverging more quickly relative to TPR2 and TPR4 possibly accounting for the functional diversity that has been reported among well characterized family members. Fourth, NASP paralogs found among ray finned fish potentially represent separate functional entities with NASP2 acquiring an NNR domain. 
We have suggested that an evolutionary conserved $\mathrm{H} 3 / \mathrm{H} 4$ transport pathway exists that most likely was also present in the LECA. Proteins including HSPs, NASP, Asf1 and Imp $\beta$ s appear to be fundamental components in this pathway. However, several other protein factors have also been shown to function in the $\mathrm{H} 3 / \mathrm{H} 4$ transport. For example, Codanin-1 has been shown to negatively regulate the influx of $\mathrm{H} 3 / \mathrm{H} 4$ by physically interacting with Asf1 in humans (Ask et al. 2012). Exploring the evolutionary histories of these additional protein factors such as Hat1/Hat2 and Codanin-1 should help providing a more comprehensive view of the $\mathrm{H} 3 / \mathrm{H} 4$ transport pathway. It is also worth mentioning here that mutations in Codanin-1 gene $(C D A N 1)$ result in a severe blood disorder known as congenital dyserythropoietic anemia type I (Dgany et al. 2002). Thus it will be of general interest to explore the evolutionary history of Codanin-1. 


\section{Appendices}

\section{Appendix 1- Accession numbers}

\subsection{T. thermophila accession numbers}

\begin{tabular}{|l|l|l|}
\hline$\#$ & TTHERM\# & Protein \\
\hline 1 & TTHERM_01014770 & Nrp1 \\
\hline 2 & TTHERM_00219420 & Cac2 \\
\hline 3 & TTHERM_00046760 & Hat1 \\
\hline 4 & TTHERM_00688660 & Hat2 \\
\hline 5 & TTHERM_00046490 & Hira \\
\hline 6 & TTHERM_00442300 & Asf1 \\
\hline
\end{tabular}

\subsection{Accession numbers}

\begin{tabular}{|l|l|}
\hline Species & Accession \\
\hline Homo sapiens & $\begin{array}{l}\text { NP_689511.2 (NASP); NP_003316.3 (Hira); Q09028 } \\
\text { (RBBP4); O14929 (Hat1); Q16576 (RBBP7) }\end{array}$ \\
\hline Xenopus laevis & AAI70018.1 (N1/N2); \\
\hline Schizosaccharomyces pombe & NP_595313.1 (Sim3); NP_596575.1 (Hip1) \\
\hline Saccharomyces cerevisiae & $\begin{array}{l}\text { NP_013078.1 (Hif1); P32479(Hir1); Q04199.1 (Cac2); } \\
\text { NP_009754.1(Cac2); NP_015324.1(Hat1); NP_010858.3 } \\
\text { (Hat2) }\end{array}$ \\
\hline Paramecium tetraurelia & XP_001456110.1 (putative NASP); \\
\hline Mus musculus & NP_034565.2 (Hira) \\
\hline Candida albicans & XP_002420628.1 (putative Cac2) \\
\hline Caenorhabditis elegans & Q21484 (Hat1) \\
\hline
\end{tabular}


Appendix 2- A: ClustalX default color legends

\begin{tabular}{|c|c|c|}
\hline $\begin{array}{c}\text { Residue at } \\
\text { position }\end{array}$ & $\begin{array}{c}\text { Applied } \\
\text { Colour }\end{array}$ & $\{$ Threshhold, Residue group $\}$ \\
\hline A,I,L,M,F,W,V & BLUE & $\{+60 \%$, WLVIMAFCHP $\}$ \\
\hline R,K & RED & $\{+60 \%, \mathrm{KR}\},\{+80 \%, \mathrm{~K}, \mathrm{R}, \mathrm{Q}\}$ \\
\hline $\mathrm{N}$ & GREEN & $\{+50 \%, \mathrm{~N}\},\{+85 \%, \mathrm{~N}, \mathrm{Y}\}$ \\
\hline $\mathrm{C}$ & BLUE & $\{100 \%, \mathrm{WLVIMAFCHP}\}$ \\
\hline $\mathrm{C}$ & PINK & $\{+60 \%, \mathrm{KR}\},\{+50 \%, \mathrm{QE}\},\{+85 \%, \mathrm{Q}, \mathrm{E}, \mathrm{K}, \mathrm{R}\}$ \\
\hline $\mathrm{Q}$ & GREEN & $\{+60 \%, \mathrm{KR}\},\{+50 \%, \mathrm{QE}\},\{+85 \%, \mathrm{E}, \mathrm{Q}, \mathrm{D}\}$ \\
\hline $\mathrm{E}$ & MAGENTA & $\{+0 \%, \mathrm{G}\}$ \\
\hline $\mathrm{D}$ & MAGENTA & $\{+60 \%, \mathrm{KR}\},\{+85 \%, \mathrm{~K}, \mathrm{R}, \mathrm{Q}\},\{+50 \%, \mathrm{ED}\}$ \\
\hline $\mathrm{G}$ & ORANGE & $\{+60 \%, \mathrm{WLVIMAFCHP}\},\{+85 \%$, \\
\hline $\mathrm{W}, \mathrm{Y}$ & CYAN & $\{+\mathrm{A}, \mathrm{C}, \mathrm{P}, \mathrm{Q}, \mathrm{F}, \mathrm{H}, \mathrm{I}, \mathrm{L}, \mathrm{M}, \mathrm{V}\}$ \\
\hline $\mathrm{P}$ & YELLOW & $\{+0 \%, \mathrm{P}\}$ \\
\hline S,T & GREEN & $\{+60 \%, \mathrm{WLVIMAFCHP}\},\{+50 \%, \mathrm{TS}\},\{+85 \%, \mathrm{~S}, \mathrm{~T}\}$ \\
\hline
\end{tabular}

Appendix 2- B: Acetylated Lysine amino acid

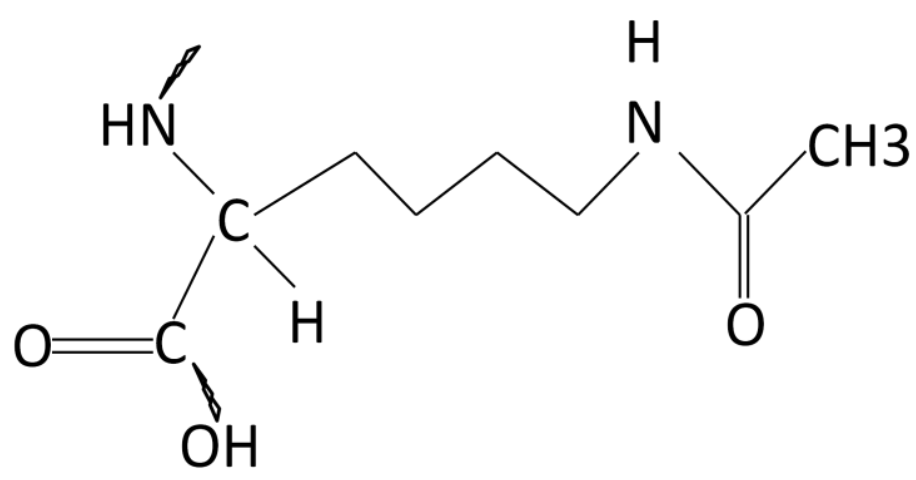


Appendix 3- Buffer recipes

\begin{tabular}{|c|c|}
\hline Item & Composition \\
\hline $1 \%$ Agarose Gel (w/v) (50ml) & $\begin{array}{l}0.5 \mathrm{~g} \text { agarose } \\
50 \mathrm{ml} 1 \mathrm{xTBE} \\
5 \mu \mathrm{l} \text { ethidium bromide }(\mathrm{EtBr}) \\
(10 \mathrm{mg} / \mathrm{ml})\end{array}$ \\
\hline 0.5M Ammonium Hydroxide $\left(\mathrm{NH}_{4} \mathrm{OH}\right)$ & $\begin{array}{l}1 \mathrm{ml} 14.5 \mathrm{M} \mathrm{NH}_{4} \mathrm{OH} \\
28 \mathrm{ml} \mathrm{ddH}_{2} \mathrm{O}\end{array}$ \\
\hline $10 \%$ APS (Ammonium persulfate) (w/v) & 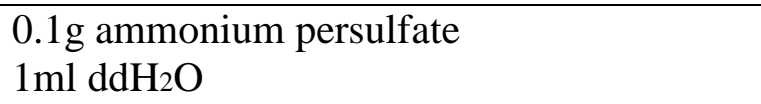 \\
\hline AP Lysis Buffer & 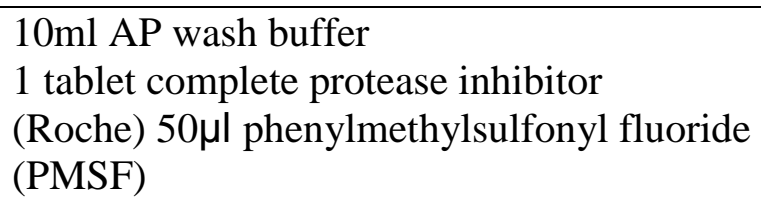 \\
\hline AP Wash Buffer & 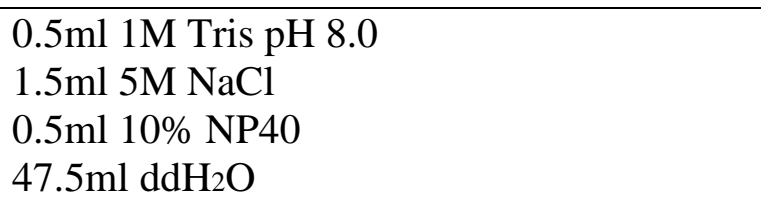 \\
\hline $1 \mathrm{M} \mathrm{CaCl}_{2}(1 \mathrm{~L})$ & $\begin{array}{l}\text { Determine which of the following } \\
\text { hydrated forms is available: } \\
\mathrm{CaCl} 2=110.99 \mathrm{~g} / \mathrm{mol} \\
\mathrm{CaCl} 2.2 \mathrm{H} 2 \mathrm{O} \text { (Dihydrate) }= \\
147.02 \mathrm{~g} / \mathrm{mol} \\
\mathrm{CaCl} 2.4 \mathrm{H} 2 \mathrm{O} \text { (Tetrahydrate) }= \\
183.04 \mathrm{~g} / \mathrm{mol} \\
\mathrm{CaCl} 2.6 \mathrm{H} 2 \mathrm{O}(\text { Hexahydrate})= \\
219.08 \mathrm{~g} / \mathrm{mol} \\
\text { To } 1 \text { mole of } \mathrm{CaCl} 2 \text { add ddH} 20 \text { to } 1 \mathrm{~L}\end{array}$ \\
\hline $2 \mathrm{mM} \mathrm{CaCl} 2 / 20 \mathrm{mM}$ Tris & $\begin{array}{l}100 \mu \mathrm{l} 1 \mathrm{M} \mathrm{CaCl} 2 \\
1 \mathrm{ml} 1 \mathrm{M} \mathrm{Tris} \mathrm{pH} 8.0 \\
48.9 \mathrm{ml} \mathrm{ddH}_{2} \mathrm{O}\end{array}$ \\
\hline $\begin{array}{l}\text { 0.5M EDTA, Iron (III) Sodium Salt } \mathrm{pH} \\
8.0(500 \mathrm{ml})\end{array}$ & $\begin{array}{l}91.78 \mathrm{~g} \mathrm{Na} \text { EDTA }(367.1 \mathrm{~g} / \mathrm{mol}) \\
\mathrm{ddH}_{2} \mathrm{O} \text { to } 500 \mathrm{ml} \text {, pH to } 8.0 \text { with } \\
\mathrm{NaOH}^{2}\end{array}$ \\
\hline 2x Lysis Buffer $(50 \mathrm{ml})$ & $\begin{array}{l}2 \mathrm{ml} 1 \mathrm{M} \text { Tris pH } 8.0 \\
50 \mu \mathrm{l} 1 \mathrm{M} \mathrm{MgCl}_{2} \\
42 \mathrm{ml} \mathrm{ddH} 2 \mathrm{O} ; 6 \mathrm{ml}_{5} 5 \mathrm{M} \mathrm{NaCl}\end{array}$ \\
\hline $1 \mathrm{M} \mathrm{MgCl}_{2}(\mathrm{M} . \mathrm{W} .=203.3 \mathrm{~g} / \mathrm{mol})(100 \mathrm{ml})$ & $20.33 \mathrm{~g} \mathrm{MgCl}_{2} ; \mathrm{ddH}_{2} \mathrm{O}$ to $100 \mathrm{ml}$ \\
\hline $1 \%$ Milk Solution $(50 \mathrm{ml})$ & $\begin{array}{l}\text { 10ml 5\% milk solution } \\
40 \mathrm{ml} 1 \mathrm{x} \text { PBS }\end{array}$ \\
\hline $\begin{array}{l}5 \% \text { Milk Solution (BLOTTO) (w/v) } \\
(100 \mathrm{ml})\end{array}$ & $\begin{array}{l}\text { 5g skim milk powder } \\
100 \mathrm{ml} \mathrm{PBS}\end{array}$ \\
\hline 100mM NaCl Wash Buffer (IPP100) & $\begin{array}{l}500 \mu \mathrm{l} 1 \mathrm{M} \text { Tris pH } 8.0 \\
1 \mathrm{ml} 5 \mathrm{M} \mathrm{NaCl} \\
500 \mu \mathrm{l} 10 \% \mathrm{NP}-40 \\
48 \mathrm{ml} \mathrm{ddH}_{2} \mathrm{O}\end{array}$ \\
\hline
\end{tabular}




\begin{tabular}{|c|c|}
\hline 300mM NaCl Wash Buffer (IPP300) & 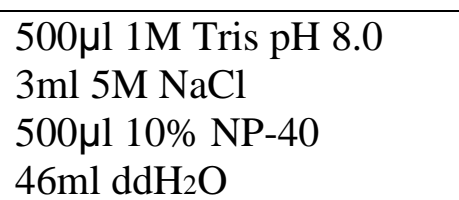 \\
\hline $5 \mathrm{M} \mathrm{NaCl}(500 \mathrm{ml})$ & $\begin{array}{l}146.1 \mathrm{~g} \mathrm{NaCl} \\
\text { ddH20 to } 500 \mathrm{ml}\end{array}$ \\
\hline $10 \%$ NP-40 (v/v) & 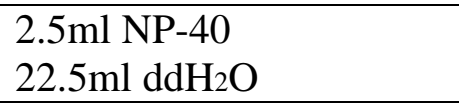 \\
\hline 10x PBS pH 7.3 (1L) & $\begin{array}{l}82 \mathrm{~g} \mathrm{NaCl} \\
2.64 \mathrm{~g} \mathrm{NaH}_{2} \mathrm{PO}_{4} \\
16 \mathrm{~g} \mathrm{Na} 2 \mathrm{HPO}_{4} \\
\text { ddH20 to } 1 \mathrm{~L}, \mathrm{pH} 7.3\end{array}$ \\
\hline 1x PBST (500ml) & $\begin{array}{l}500 \mathrm{ml} 1 \times \text { PBS } \\
250 \mu 1 \text { Tween } 20\end{array}$ \\
\hline 100mM PMSF (10ml) & $\begin{array}{l}0.1742 \mathrm{~g} \text { PMSF } \\
10 \mathrm{ml} \text { isopropanol }\end{array}$ \\
\hline Ponceau $(0.1 \% \mathrm{w} / \mathrm{v})(1 \mathrm{~L})$ & $\begin{array}{l}1 \mathrm{~g} \text { Ponceau } \mathrm{S} \\
50 \mathrm{ml} \text { acetic acid } \\
\mathrm{ddH} 20 \text { to } 1 \mathrm{~L}\end{array}$ \\
\hline 2x SDS Laemmli Sample Buffer & $\begin{array}{l}3 \mathrm{~g} \text { SDS } \\
5 \mathrm{ml} \text { beta-mercaptoethanol } \\
10 \mathrm{ml} 100 \% \text { glycerol } \\
6 \mathrm{ml} 2 \mathrm{M} \text { Tris-HCL pH } 6.8 \\
50 \mathrm{mg} \text { bromophenol blue } \\
\text { ddH } 20 \text { to } 100 \mathrm{ml}\end{array}$ \\
\hline SPP (1L) & $\begin{array}{l}\text { 60mg sequestrin (Sigma) } \\
2 \mathrm{~g} \text { bacto yeast extract } \\
20 \mathrm{~g} \text { proteose peptone } \\
4 \mathrm{~g} \text { glucose } \\
\text { ddH20 to } 1 \mathrm{~L}\end{array}$ \\
\hline $5 \%$ Stacking Gel $(5 \mathrm{ml})$ & $\begin{array}{l}3.5 \mathrm{ml} \mathrm{ddH}_{2} \mathrm{O} \\
0.625 \mathrm{ml} 1 \mathrm{M} \text { Tris } \mathrm{pH} 6.8 \\
0.95 \mathrm{ml} \text { acrylamide } 29: 1 \\
0.05 \mathrm{ml} 10 \% \text { SDS } \\
3.75 \mu 1 \text { TEMED } \\
31.25 \mu 110 \% \text { APS }\end{array}$ \\
\hline Tetrahymena Lysis Solution $(500 \mathrm{ml})$ & $\begin{array}{l}210 \mathrm{~g} \text { urea } \\
35 \mathrm{ml} 5 \mathrm{M} \mathrm{NaCl} \\
5 \mathrm{ml} 1 \mathrm{M} \text { Tris pH } 7.4 \\
10 \mathrm{ml} 0.5 \mathrm{M} \mathrm{EDTA} \\
50 \mathrm{ml} 10 \% \text { SDS } \\
\mathrm{ddH}_{2} \mathrm{O} \text { to } 500 \mathrm{ml}\end{array}$ \\
\hline 1x TEV Cleavage Buffer & 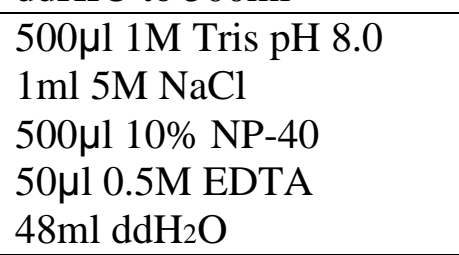 \\
\hline 10mM Tris pH 7.4 (1L) & $1.21 \mathrm{~g}$ Tris \\
\hline
\end{tabular}




\begin{tabular}{|l|l|}
\hline & $\mathrm{ddH}_{2} \mathrm{O}$ to $1 \mathrm{~L}, \mathrm{pH}$ to 7.4 \\
\hline YT Media (1L) & $10 \mathrm{~g}$ bacto-tryptone \\
& $5 \mathrm{~g}$ yeast extract \\
& $5 \mathrm{~g} \mathrm{NaCl}$ \\
& $15 \mathrm{~g}$ agar for plates \\
& $1 \mathrm{LddH}_{2} 0$ \\
\hline
\end{tabular}

\section{Appendix 4- Primers}

\section{1- Sequencing primer Sequence}

M13R 5'-CAGGAAACAGCTATGAC-3'

HN111 5'-TATCATCATCATCTTTGTAATCAATATC-3'

M13F 5'-TGTAAAACGACGGCCAGT-3'

\section{2 - PCR primers to amplify gene sequences for molecular cloning}

\section{Hat1:}

Upstream Forward: 5'CCCGGTACCTTGAATACTCTTATGAAAAAAGATGA 3'

Upstream Reverse: 5'CCCCTCGAG AATCTTGAGTTTTTAAATTACATTATT 3'

Downstream Forward: 5' CCCGCGGCCGC CTAATTAAGATTGTGTCTATATTTATT 3'

Downstream Reverse: 5' CCCGAGCTCTGTTCTATT TGCTAATCTTAAGCTTAAGAT3'

\section{Hat2:}

Upstream Forward: 5'CCCGGTACCAAATAA AACTTATTAAAATTT TTA ATT AGG 3'

Upstream Reverse: 5'CCCCTCGAG GTC TTT CATTAC TTCATC ATC AGTATCATC 3'

Downstream For: 5'CCCCGCGGCCGCTTTGATTTACTCAGTTACTTAATTAAAAAT3'

Downstream Reverse: 5'CCCGAGCTCATCAACGATCAATGTACAGCTGGTGTTTCT 3' Nrp1:

Upstream Forward: 5' CCCGGTACCTAAAGATGAAAATGTAGAAATTAGAGATGA 3' 
Upstream Reverse: 5' CCCCTCGAGATCCTTTTTGAGCATTTTATCTGGGTTTCC 3'

Downstream For: 5'CCCGCGGCCGCAAGAAGAAATAAATAAATATTTTTAGAGTA3'

Downstream Reverse: 5'CCCGAGCTCAGGATTGTATAAGGGTATTAGATGTAT3'

Cac2:

Upstream Forward: 5' CCCGGTACCAATTTTTTATGAGAAATTTTTTTATCAGCA3'

Upstream Reverse: 5' CCCCTCGAGGTATTTCTTAATAACTGCAGGAATAATTAC3'

Downstream Forward:

5'CCCGCGGCCGCAAATAGTATATATTTAAAAACCATCCATAATAG3'

Downstream Reverse: 5' CCCGAGCTCCCCTATTATTAACACTTAATAATCAATGAA3'

Hira:

Upstream Forward: 5' CCCGGTACCTAATGCTAAAGAGATATTATGGAATGACTG 3'

Upstream Reverse: 5' CCC CTCGAG ATTAGATTTTCCTTTAAATTAAATGATTTC 3'

Downstream Forward: 5' CCCC GCGGCCGC AAA ATT CTC TTT AACTAAATAATA TCT TTA 3 ,

Downstream Reverse: 5' CCCC GAGCTC AAC AGC TTC AGT TCT ATC AGT GAT ATT GTT 3' 


\section{Appendix 5- Protein ladder}

PiNK plus prestained protein ladder scale (Frogga Bio)

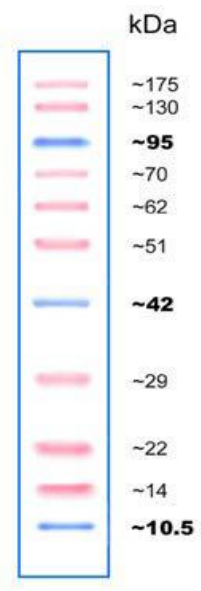

Tris-Glycine

$15 \%$

Spectra multicolor broad range protein ladder (Thermo Scientific)

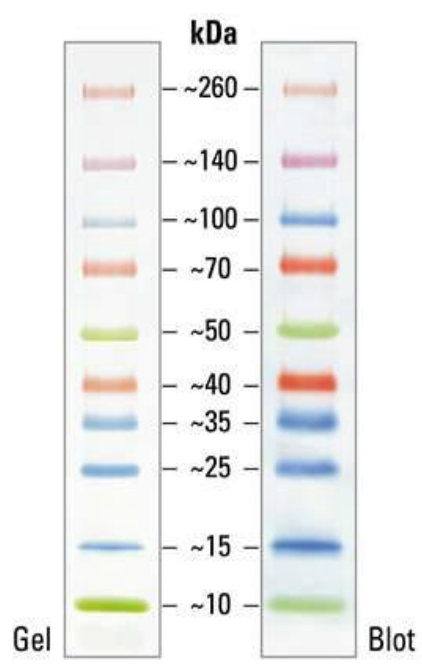




\section{References (Part1)}

Aalfs JD, Kingston RE. 2000. What does "chromatin remodeling" mean? Trends Biochem. Sci. 25:548-555.

Abascal F, Corpet A, Gurard-Levin ZA, Juan D, Ochsenbein F, Rico D, Valencia A, Almouzni G. 2013. Subfunctionalization via adaptive evolution influenced by genomic context: the case of histone chaperones ASF1a and ASF1b. Mol. Biol. Evol. 30:18531866.

Adkins MW, Williams SK, Linger J, Tyler JK. 2007. Chromatin disassembly from the PHO5 promoter is essential for the recruitment of the general transcription machinery and coactivators. Mol. Cell. Biol. 27:6372-6382.

Adl SM, Simpson AGB, Lane CE, et al. 2012. The revised classification of eukaryotes. J. Eukaryot. Microbiol. 59:429-493.

Ahmad A, Kikuchi H, Takami Y, Nakayama T. 2005. Different roles of N-terminal and Cterminal halves of HIRA in transcription regulation of cell cycle-related genes that contribute to control of vertebrate cell growth. J. Biol. Chem. 280:32090-32100.

Ahmad K, Henikoff S. 2002. The Histone Variant H3.3 Marks Active Chromatin by Replication-Independent Nucleosome Assembly. Mol. Cell 9:1191-1200.

Ai X, Parthun MR. 2004. The nuclear Hat1p/Hat2p complex: a molecular link between type B histone acetyltransferases and chromatin assembly. Mol. Cell 14:195-205.

Akematsu T, Endoh H. 2010. Role of apoptosis-inducing factor (AIF) in programmed nuclear death during conjugation in Tetrahymena thermophila. BMC Cell Biol. 11:13.

Alekseev OM, Bencic DC, Richardson RT, Widgren EE, O'Rand MG. 2003. Overexpression of the Linker histone-binding protein tNASP affects progression through the cell cycle. J. Biol. Chem. 278:8846-8852.

Alekseev OM, Richardson RT, Pope MR, O'Rand MG. 2005. Mass spectrometry identification of NASP binding partners in HeLa cells. Proteins Struct. Funct. Genet. $61: 1-5$.

Ali-Fehmi R, Chatterjee M, Ionan A, et al. 2010. Analysis of the expression of human tumor antigens in ovarian cancer tissues. Cancer Biomark.

Allis CD, Chicoine LG, Richman R, Schulman IG. 1985. Deposition-related histone acetylation in micronuclei of conjugating Tetrahymena. Proc. Natl. Acad. Sci. U. S. A. 82:8048-8052.

Allis CD, Wiggins JC. 1984. Histone rearrangements accompany nuclear differentiation and dedifferentiation in Tetrahymena. Dev. Biol. 101:282-294. 
Alvarez F, Muñoz F, Schilcher P, Imhof A, Almouzni G, Loyola A. 2011. Sequential establishment of marks on soluble histones H3 and H4. J. Biol. Chem. 286:1771417721.

Aronica L, Bednenko J, Noto T, DeSouza L V, Siu KWM, Loidl J, Pearlman RE, Gorovsky MA, Mochizuki K. 2008. Study of an RNA helicase implicates small RNA-noncoding RNA interactions in programmed DNA elimination in Tetrahymena. Genes Dev. 22:2228-2241.

Ask K, Jasencakova Z, Menard P, Feng Y, Almouzni G, Groth A. 2012. Codanin-1, mutated in the anaemic disease CDAI, regulates Asf1 function in S-phase histone supply. EMBO J. 31:2013-2023.

Austerberry CF, Snyder RO, Yao MC. 1989. Sequence microheterogeneity is generated at junctions of programmed DNA deletions in Tetrahymena thermophila. Nucleic Acids Res. 17:7263-7272.

Balaji S, Iyer LM, Aravind L. 2009. HPC2 and ubinuclein define a novel family of histone chaperones conserved throughout eukaryotes. Mol. Biosyst. 5:269-275.

Bannister AJ, Kouzarides T. 2011. Regulation of chromatin by histone modifications. Cell Res. 21:381-395.

Bao Y, Shen X. 2006. Asf1, a loveseat for a histone couple. Cell 127:458-460.

Barman HK, Takami Y, Nishijima H, Shibahara K, Sanematsu F, Nakayama T. 2008. Histone acetyltransferase-1 regulates integrity of cytosolic histone H3-H4 containing complex. Biochem. Biophys. Res. Commun. 373:624-630.

Bartek J, Bartkova J, Lukas J. 1997. The retinoblastoma protein pathway in cell cycle control and cancer. Exp. Cell Res. 237:1-6.

Bernas T, Brutkowski W, Zarębski M, Dobrucki J. 2014. Spatial heterogeneity of dynamics of H1 linker histone. Eur. Biophys. J. 43:287-300.

Bernstein E, Allis CD. 2005. RNA meets chromatin. Genes Dev. 19:1635-1655.

Biterge B, Schneider R. 2014. Histone variants: key players of chromatin. Cell Tissue Res. 356:457-466.

Blackwell C, Martin KA, Greenall A, Pidoux A, Allshire RC, Whitehall SK. 2004. The Schizosaccharomyces pombe HIRA-like protein Hip1 is required for the periodic expression of histone genes and contributes to the function of complex centromeres. Mol. Cell. Biol. 24:4309-4320.

Brownell JE, Zhou J, Ranalli T, Kobayashi R, Edmondson DG, Roth SY, Allis CD. 1996. Tetrahymena histone acetyltransferase A: a homolog to yeast Gcn5p linking histone acetylation to gene activation. Cell 84:843-851. 
Bruns PJ, Cassidy-Hanley D. 2000. Biolistic transformation of macro- and micronuclei. Methods Cell Biol. 62:501-512.

Burgess RJ, Zhang Z. 2013. Histone chaperones in nucleosome assembly and human disease. Nat. Struct. Mol. Biol. 20:14-22.

Campos EI, Fillingham J, Li G, et al. 2010. The program for processing newly synthesized histones H3.1 and H4. Nat. Struct. Mol. Biol. 17:1343-1351.

Cervantes MD, Xi X, Vermaak D, Yao M-C, Malik HS. 2006. The CNA1 histone of the ciliate Tetrahymena thermophila is essential for chromosome segregation in the germline micronucleus. Mol. Biol. Cell 17:485-497.

Chicoine LG, Richman R, Cook RG, Gorovsky MA, Allis CD. 1987. A single histone acetyltransferase from Tetrahymena macronuclei catalyzes deposition-related acetylation of free histones and transcription-related acetylation of nucleosomal histones. J. Cell Biol. 105:127-135.

Choi H, Liu G, Mellacheruvu D, Tyers M, Gingras A-C, Nesvizhskii AI. 2012. Analyzing protein-protein interactions from affinity purification-mass spectrometry data with SAINT. Curr. Protoc. Bioinformatics Chapter 8:Unit8.15.

Coggill P, Finn RD, Bateman A. 2008. Identifying protein domains with the Pfam database. Curr. Protoc. Bioinformatics Chapter 2:Unit 2.5.

Cook AJL, Gurard-Levin ZA, Vassias I, Almouzni G. 2011. A Specific Function for the Histone Chaperone NASP to Fine-Tune a Reservoir of Soluble H3-H4 in the Histone Supply Chain. Mol. Cell 44:918-927.

Cui B, Liu Y, Gorovsky MA. 2006. Deposition and function of histone H3 variants in Tetrahymena thermophila. Mol. Cell. Biol. 26:7719-7730.

D’Andrea LD, Regan L. 2003. TPR proteins: the versatile helix. Trends Biochem. Sci. 28:655-662.

Das C, Lucia MS, Hansen KC, Tyler JK. 2009. CBP/p300-mediated acetylation of histone H3 on lysine 56. Nature 459:113-117.

Dephoure N, Zhou C, Villén J, Beausoleil SA, Bakalarski CE, Elledge SJ, Gygi SP. 2008. A quantitative atlas of mitotic phosphorylation. Proc. Natl. Acad. Sci. U. S. A. 105:1076210767.

Dilworth SM, Black SJ, Laskey RA. 1987. Two complexes that contain histones are required for nucleosome assembly in vitro: role of nucleoplasmin and N1 in Xenopus egg extracts. Cell 51:1009-1018.

Dunleavy EM, Pidoux AL, Monet M, Bonilla C, Richardson W, Hamilton GL, Ekwall K, McLaughlin PJ, Allshire RC. 2007. A NASP (N1/N2)-related protein, Sim3, binds 
CENP-A and is required for its deposition at fission yeast centromeres. Mol. Cell 28:1029-1044.

Dutnall RN, Tafrov ST, Sternglanz R, Ramakrishnan V. 1998. Structure of the histone acetyltransferase Hat1: a paradigm for the GCN5-related N-acetyltransferase superfamily. Cell 94:427-438.

Eisen JA, Coyne RS, Wu M, et al. 2006. Macronuclear genome sequence of the ciliate Tetrahymena thermophila, a model eukaryote. PLoS Biol. 4:e286.

Emili A, Schieltz DM, Yates JR, Hartwell LH. 2001. Dynamic interaction of DNA damage checkpoint protein Rad53 with chromatin assembly factor Asf1. Mol. Cell 7:13-20.

Esterlechner J, Reichert N, Iltzsche F, Krause M, Finkernagel F, Gaubatz S. 2013. LIN9, a subunit of the DREAM complex, regulates mitotic gene expression and proliferation of embryonic stem cells. PLoS One 8:e62882.

Fan Q, Yao MC. 2000. A long stringent sequence signal for programmed chromosome breakage in Tetrahymena thermophila. Nucleic Acids Res. 28:895-900.

Fillingham J, Greenblatt JF. 2008. A histone code for chromatin assembly. Cell 134:206208.

Fillingham J, Kainth P, Lambert J-P, et al. 2009. Two-color cell array screen reveals interdependent roles for histone chaperones and a chromatin boundary regulator in histone gene repression. Mol. Cell 35:340-351.

Fillingham J, Recht J, Silva AC, et al. 2008. Chaperone control of the activity and specificity of the histone H3 acetyltransferase Rtt109. Mol. Cell. Biol. 28:4342-4353.

Finn RM, Browne K, Hodgson KC, Ausió J. 2008. sNASP, a histone H1-specific eukaryotic chaperone dimer that facilitates chromatin assembly. Biophys. J. 95:1314-1325.

Finn RM, Ellard K, Eirin Lopez JM, Ausio J. 2012. Vertebrate nucleoplasmin and NASP: egg histone storage proteins with multiple chaperone activities. FASEB J.

Fischle W, Wang Y, Allis CD. 2003. Histone and chromatin cross-talk. Curr. Opin. Cell Biol. 15:172-183.

Formosa T, Ruone S, Adams MD, Olsen AE, Eriksson P, Yu Y, Rhoades AR, Kaufman PD, Stillman DJ. 2002. Defects in SPT16 or POB3 (yFACT) in Saccharomyces cerevisiae cause dependence on the Hir/Hpc pathway: polymerase passage may degrade chromatin structure. Genetics 162:1557-1571.

Gaertig J, Gorovsky MA. 1992. Efficient mass transformation of Tetrahymena thermophila by electroporation of conjugants. Proc. Natl. Acad. Sci. U. S. A. 89:9196-9200. 
Gaertig J, Gu L, Hai B, Gorovsky MA. 1994. High frequency vector-mediated transformation and gene replacement in Tetrahymena. Nucleic Acids Res. 22:53915398.

Garcia BA, Hake SB, Diaz RL, et al. 2007. Organismal differences in post-translational modifications in histones H3 and H4. J. Biol. Chem. 282:7641-7655.

Garg J, Lambert JP, Karsou A, et al. 2013. Conserved Asf1-importin ?? physical interaction in growth and sexual development in the ciliate Tetrahymena thermophila. J. Proteomics 94:311-326.

Ge Z, Nair D, Guan X, Rastogi N, Freitas MA, Parthun MR. 2013. Sites of acetylation on newly synthesized histone $\mathrm{H} 4$ are required for chromatin assembly and DNA damage response signaling. Mol. Cell. Biol. 33:3286-3298.

Ge Z, Wang H, Parthun MR. 2011. Nuclear Hat1p complex (NuB4) components participate in DNA repair-linked chromatin reassembly. J. Biol. Chem. 286:16790-16799.

Gérard A, Koundrioukoff S, Ramillon V, Sergère J-C, Mailand N, Quivy J-P, Almouzni G. 2006. The replication kinase Cdc7-Dbf4 promotes the interaction of the p150 subunit of chromatin assembly factor 1 with proliferating cell nuclear antigen. EMBO Rep. 7:817823.

Godiska R, James C, Yao MC. 1993. A distant 10-bp sequence specifies the boundaries of a programmed DNA deletion in Tetrahymena. Genes Dev. 7:2357-2365.

Green EM, Antczak AJ, Bailey AO, Franco AA, Wu KJ, Yates JR, Kaufman PD. 2005. Replication-independent histone deposition by the HIR complex and Asf1. Curr. Biol. 15:2044-2049.

Greenall A, Williams ES, Martin KA, Palmer JM, Gray J, Liu C, Whitehall SK. 2006. Hip3 interacts with the HIRA proteins Hip1 and SIm9 and is required for transcriptional silencing and accurate chromosome segregation. J. Biol. Chem. 281:8732-8739.

Greider CW, Blackburn EH. 1985. Identification of a specific telomere terminal transferase activity in Tetrahymena extracts. Cell 43:405-413.

Greider CW, Blackburn EH. 1987. The telomere terminal transferase of Tetrahymena is a ribonucleoprotein enzyme with two kinds of primer specificity. Cell 51:887-898.

Groth A, Corpet A, Cook AJL, Roche D, Bartek J, Lukas J, Almouzni G. 2007. Regulation of replication fork progression through histone supply and demand. Science 318:19281931.

Grzenda A, Lomberk G, Zhang J-S, Urrutia R. 2009. Sin3: master scaffold and transcriptional corepressor. Biochim. Biophys. Acta 1789:443-450. 
Han J, Zhou H, Horazdovsky B, Zhang K, Xu R-M, Zhang Z. 2007. Rtt109 acetylates histone $\mathrm{H} 3$ lysine 56 and functions in DNA replication. Science 315:653-655.

Hennig L, Bouveret R, Gruissem W. 2005. MSI1-like proteins: an escort service for chromatin assembly and remodeling complexes. Trends Cell Biol. 15:295-302.

Hoek M, Stillman B. 2003. Chromatin assembly factor 1 is essential and couples chromatin assembly to DNA replication in vivo. Proc. Natl. Acad. Sci. U. S. A. 100:12183-12188.

Horn PJ, Peterson CL. 2006. Heterochromatin assembly: a new twist on an old model. Chromosome Res. 14:83-94.

Howard-Till RA, Yao M-C. 2006. Induction of gene silencing by hairpin RNA expression in Tetrahymena thermophila reveals a second small RNA pathway. Mol. Cell. Biol. 26:8731-8742.

Jackson V, Shires A, Tanphaichitr N, Chalkley R. 1976. Modifications to histones immediately after synthesis. J. Mol. Biol. 104:471-483.

Jiang J, Benson E, Bausek N, Doggett K, White-Cooper H. 2007. Tombola, a tesmin/TSO1family protein, regulates transcriptional activation in the Drosophila male germline and physically interacts with always early. Development 134:1549-1559.

Jullien J, Astrand C, Szenker E, Garrett N, Almouzni G, Gurdon JB. 2012. HIRA dependent H3.3 deposition is required for transcriptional reprogramming following nuclear transfer to Xenopus oocytes. Epigenetics Chromatin 5:17.

Kaufman PD, Cohen JL, Osley MA. 1998. Hir proteins are required for position-dependent gene silencing in Saccharomyces cerevisiae in the absence of chromatin assembly factor I. Mol. Cell. Biol. 18:4793-4806.

Kaufman PD, Kobayashi R, Kessler N, Stillman B. 1995. The p150 and p60 subunits of chromatin assembly factor I: a molecular link between newly synthesized histones and DNA replication. Cell 81:1105-1114.

Kaufman PD, Kobayashi R, Stillman B. 1997. Ultraviolet radiation sensitivity and reduction of telomeric silencing in Saccharomyces cerevisiae cells lacking chromatin assembly factor-I. Genes Dev. 11:345-357.

Keck KM, Pemberton LF. 2012. Histone chaperones link histone nuclear import and chromatin assembly. Biochim. Biophys. Acta 1819:277-289.

Kelley LA, Sternberg MJE. 2009. Protein structure prediction on the Web: a case study using the Phyre server. Nat. Protoc. 4:363-371.

Kleinschmidt JA, Fortkamp E, Krohne G, Zentgraf H, Franke WW. 1985. Co-existence of two different types of soluble histone complexes in nuclei of Xenopus laevis oocytes. J. Biol. Chem. 260:1166-1176. 
De Koning L, Corpet A, Haber JE, Almouzni G. 2007. Histone chaperones: an escort network regulating histone traffic. Nat. Struct. Mol. Biol. 14:997-1007.

Kruger K, Grabowski PJ, Zaug AJ, Sands J, Gottschling DE, Cech TR. 1982. Self-splicing RNA: autoexcision and autocyclization of the ribosomal RNA intervening sequence of Tetrahymena. Cell 31:147-157.

Kuo MH, Brownell JE, Sobel RE, Ranalli TA, Cook RG, Edmondson DG, Roth SY, Allis CD. 1996. Transcription-linked acetylation by Gcn5p of histones H3 and H4 at specific lysines. Nature 383:269-272.

Larkin MA, Blackshields G, Brown NP, et al. 2007. Clustal W and Clustal X version 2.0. Bioinformatics 23:2947-2948.

Latreille D, Bluy L, Benkirane M, Kiernan RE. 2014. Identification of histone 3 variant 2 interacting factors. Nucleic Acids Res. 42:3542-3550.

Le S, Davis C, Konopka JB, Sternglanz R. 1997. Two new S-phase-specific genes from Saccharomyces cerevisiae. Yeast 13:1029-1042.

Letunic I, Doerks T, Bork P. 2012. SMART 7: recent updates to the protein domain annotation resource. Nucleic Acids Res. 40:D302-5.

Li B, Carey M, Workman JL. 2007. The role of chromatin during transcription. Cell 128:707-719.

Li Q, Burgess R, Zhang Z. 2012. All roads lead to chromatin: Multiple pathways for histone deposition. Biochim. Biophys. Acta 1819:238-246.

Li Y, Zhang L, Liu T, et al. 2014. Hat2p recognizes the histone H3 tail to specify the acetylation of the newly synthesized H3/H4 heterodimer by the Hat1p/Hat2p complex. Genes Dev. 28:1217-1227.

Linger J, Tyler JK. 2005. The yeast histone chaperone chromatin assembly factor 1 protects against double-strand DNA-damaging agents. Genetics 171:1513-1522.

Liu H, Zhang M, He W, Zhu Z, Teng M, Gao Y, Niu L. 2014. Structural insights into yeast histone chaperone Hif1: a scaffold protein recruiting protein complexes to core histones. Biochem. J.

Lorain S, Demczuk S, Lamour V, Toth S, Aurias A, Roe BA, Lipinski M. 1996. Structural Organization of the WD repeat protein-encoding gene HIRA in the DiGeorge syndrome critical region of human chromosome 22. Genome Res. 6:43-50.

Luger K, Mäder AW, Richmond RK, Sargent DF, Richmond TJ. 1997. Crystal structure of the nucleosome core particle at 2.8 A resolution. Nature 389:251-260. 
Lundgren DH, Hwang S-I, Wu L, Han DK. 2010. Role of spectral counting in quantitative proteomics. Expert Rev. Proteomics 7:39-53.

Lynn DH, Doerder FP. 2012. The life and times of Tetrahymena. Methods Cell Biol. 109:927.

Ma W, Xie S, Ni M, Huang X, Hu S, Liu Q, Liu A, Zhang J, Zhang Y. 2012. MicroRNA-29a inhibited epididymal epithelial cell proliferation by targeting nuclear autoantigenic sperm protein (NASP). J. Biol. Chem. 287:10189-10199.

Ma XJ, Wu J, Altheim BA, Schultz MC, Grunstein M. 1998. Deposition-related sites $\mathrm{K} 5 / \mathrm{K} 12$ in histone $\mathrm{H} 4$ are not required for nucleosome deposition in yeast. Proc. Natl. Acad. Sci. U. S. A. 95:6693-6698.

Madireddi MT, Coyne RS, Smothers JF, Mickey KM, Yao MC, Allis CD. 1996. Pdd1p, a novel chromodomain-containing protein, links heterochromatin assembly and DNA elimination in Tetrahymena. Cell 87:75-84.

Malay AD, Umehara T, Matsubara-Malay K, Padmanabhan B, Yokoyama S. 2008. Crystal structures of fission yeast histone chaperone Asf1 complexed with the Hip1 B-domain or the Cac2 C terminus. J. Biol. Chem. 283:14022-14031.

Mariño-Ramírez L, Kann MG, Shoemaker BA, Landsman D. 2005. Histone structure and nucleosome stability. Expert Rev. Proteomics 2:719-729.

Martindale DW, Allis CD, Bruns PJ. 1982. Conjugation in Tetrahymena thermophila. A temporal analysis of cytological stages. Exp. Cell Res. 140:227-236.

Martini E, Roche DM, Marheineke K, Verreault A, Almouzni G. 1998. Recruitment of phosphorylated chromatin assembly factor 1 to chromatin after UV irradiation of human cells. J. Cell Biol. 143:563-575.

Masumoto H, Hawke D, Kobayashi R, Verreault A. 2005. A role for cell-cycle-regulated histone H3 lysine 56 acetylation in the DNA damage response. Nature 436:294-298.

Matsuoka S, Ballif BA, Smogorzewska A, et al. 2007. ATM and ATR substrate analysis reveals extensive protein networks responsive to DNA damage. Science 316:11601166.

McKittrick E, Gafken PR, Ahmad K, Henikoff S. 2004. Histone H3.3 is enriched in covalent modifications associated with active chromatin. Proc. Natl. Acad. Sci. U. S. A. 101:1525-1530.

Meggio F, Pinna LA. 2003. One-thousand-and-one substrates of protein kinase CK2? FASEB J. 17:349-368.

Mersfelder EL, Parthun MR. 2008. Involvement of Hat1p (Kat1p) catalytic activity and subcellular localization in telomeric silencing. J. Biol. Chem. 283:29060-29068. 
Miao W, Xiong J, Bowen J, et al. 2009. Microarray analyses of gene expression during the Tetrahymena thermophila life cycle. PLoS One 4:e4429.

Mochizuki K, Fine NA, Fujisawa T, Gorovsky MA. 2002. Analysis of a piwi-related gene implicates small RNAs in genome rearrangement in tetrahymena. Cell 110:689-699.

Mochizuki K, Gorovsky MA. 2004. Small RNAs in genome rearrangement in Tetrahymena. Curr. Opin. Genet. Dev. 14:181-187.

Mochizuki K, Gorovsky MA. 2005. A Dicer-like protein in Tetrahymena has distinct functions in genome rearrangement, chromosome segregation, and meiotic prophase. Genes Dev. 19:77-89.

Moggs JG, Grandi P, Quivy JP, Jónsson ZO, Hübscher U, Becker PB, Almouzni G. 2000. A CAF-1-PCNA-mediated chromatin assembly pathway triggered by sensing DNA damage. Mol. Cell. Biol. 20:1206-1218.

Murzina N V, Pei X-Y, Zhang W, et al. 2008. Structural basis for the recognition of histone H4 by the histone-chaperone RbAp46. Structure 16:1077-1085.

Murzina N, Verreault A, Laue E, Stillman B. 1999. Heterochromatin dynamics in mouse cells: interaction between chromatin assembly factor 1 and HP1 proteins. Mol. Cell 4:529-540.

Musselman CA, Kutateladze TG. 2009. PHD fingers: epigenetic effectors and potential drug targets. Mol. Interv. 9:314-323.

Nagarajan P, Ge Z, Sirbu B, et al. 2013. Histone acetyl transferase 1 is essential for mammalian development, genome stability, and the processing of newly synthesized histones H3 and H4. PLoS Genet. 9:e1003518.

Noto T, Kurth HM, Kataoka K, Aronica L, DeSouza L V, Siu KWM, Pearlman RE, Gorovsky MA, Mochizuki K. 2010. The Tetrahymena argonaute-binding protein Giw1p directs a mature argonaute-siRNA complex to the nucleus. Cell 140:692-703.

Olesen C, Møller M, Byskov AG. 2004. Tesmin transcription is regulated differently during male and female meiosis. Mol. Reprod. Dev. 67:116-126.

Orias E, Cervantes MD, Hamilton EP. 2011. Tetrahymena thermophila, a unicellular eukaryote with separate germline and somatic genomes. Res. Microbiol. 162:578-586.

Orias E. Toward sequencing the Tetrahymena genome: exploiting the gift of nuclear dimorphism. J. Eukaryot. Microbiol. 47:328-333.

Osakabe A, Tachiwana H, Matsunaga T, Shiga T, Nozawa R-S, Obuse C, Kurumizaka H. 2010. Nucleosome formation activity of human somatic nuclear autoantigenic sperm protein (sNASP). J. Biol. Chem. 285:11913-11921. 
Osley MA, Lycan D. 1987. Trans-acting regulatory mutations that alter transcription of Saccharomyces cerevisiae histone genes. Mol. Cell. Biol. 7:4204-4210.

Paoli M. 2001. Protein folds propelled by diversity. Prog. Biophys. Mol. Biol. 76:103-130.

Parthun MR, Widom J, Gottschling DE. 1996. The major cytoplasmic histone acetyltransferase in yeast: links to chromatin replication and histone metabolism. Cell 87:85-94.

Parthun MR. 2007. Hat1: the emerging cellular roles of a type B histone acetyltransferase. Oncogene 26:5319-5328.

Parthun MR. 2012. Histone acetyltransferase 1: More than just an enzyme? Biochim. Biophys. Acta 1819:256-263.

Pitts RA, Doerder FP. 1988. Genomic exclusion and other micronuclear anomalies are common in genetically defective clones of tetrahymena thermophila. Genetics 120:135143.

Polo SE, Theocharis SE, Grandin L, Gambotti L, Antoni G, Savignoni A, Asselain B, Patsouris E, Almouzni G. 2010. Clinical significance and prognostic value of chromatin assembly factor-1 overexpression in human solid tumours. Histopathology 57:716-724.

Poveda A, Pamblanco M, Tafrov S, Tordera V, Sternglanz R, Sendra R. 2004. Hif1 is a component of yeast histone acetyltransferase $\mathrm{B}$, a complex mainly localized in the nucleus. J. Biol. Chem. 279:16033-16043.

Prado F, Cortés-Ledesma F, Aguilera A. 2004. The absence of the yeast chromatin assembly factor Asf1 increases genomic instability and sister chromatid exchange. EMBO Rep. 5:497-502.

Prochasson P, Florens L, Swanson SK, Washburn MP, Workman JL. 2005. The HIR corepressor complex binds to nucleosomes generating a distinct protein/DNA complex resistant to remodeling by SWI/SNF. Genes Dev. 19:2534-2539.

Quivy J-P, Gérard A, Cook AJL, Roche D, Almouzni G. 2008. The HP1-p150/CAF-1 interaction is required for pericentric heterochromatin replication and S-phase progression in mouse cells. Nat. Struct. Mol. Biol. 15:972-979.

Quivy JP, Grandi P, Almouzni G. 2001. Dimerization of the largest subunit of chromatin assembly factor 1: importance in vitro and during Xenopus early development. EMBO J. 20:2015-2027.

Rai TS, Puri A, McBryan T, et al. 2011. Human CABIN1 is a functional member of the human HIRA/UBN1/ASF1a histone H3.3 chaperone complex. Mol. Cell. Biol. $31: 4107-4118$. 
Ramirez-Parra E, Gutierrez C. 2007. The many faces of chromatin assembly factor 1 . Trends Plant Sci. 12:570-576.

Reese BE, Bachman KE, Baylin SB, Rountree MR. 2003. The methyl-CpG binding protein MBD1 interacts with the p150 subunit of chromatin assembly factor 1. Mol. Cell. Biol. 23:3226-3236.

Reichert N, Wurster S, Ulrich T, et al. 2010. Lin9, a subunit of the mammalian DREAM complex, is essential for embryonic development, for survival of adult mice, and for tumor suppression. Mol. Cell. Biol. 30:2896-2908.

Richardson RT, Alekseev OM, Grossman G, Widgren EE, Thresher R, Wagner EJ, Sullivan KD, Marzluff WF, O’Rand MG. 2006. Nuclear autoantigenic sperm protein (NASP), a linker histone chaperone that is required for cell proliferation. J. Biol. Chem. 281:21526-21534.

Richardson RT, Batova IN, Widgren EE, Zheng LX, Whitfield M, Marzluff WF, O'Rand MG. 2000. Characterization of the histone H1-binding protein, NASP, as a cell cycleregulated somatic protein. J. Biol. Chem. 275:30378-30386.

Ridgway P, Almouzni G. 2000. CAF-1 and the inheritance of chromatin states: at the crossroads of DNA replication and repair. J. Cell Sci. 113 ( Pt 1:2647-2658.

Rolef Ben-Shahar T, Castillo AG, Osborne MJ, Borden KLB, Kornblatt J, Verreault A. 2009. Two fundamentally distinct PCNA interaction peptides contribute to chromatin assembly factor 1 function. Mol. Cell. Biol. 29:6353-6365.

Roth SY, Allis CD. 1996. Histone acetylation and chromatin assembly: a single escort, multiple dances? Cell 87:5-8.

Roy A, Yang J, Zhang Y. 2012. COFACTOR: an accurate comparative algorithm for structure-based protein function annotation. Nucleic Acids Res. 40:W471-7.

Ruiz-García AB, Sendra R, Galiana M, Pamblanco M, Pérez-Ortín JE, Tordera V. 1998. HAT1 and HAT2 proteins are components of a yeast nuclear histone acetyltransferase enzyme specific for free histone H4. J. Biol. Chem. 273:12599-12605.

Ruiz-Roig C, Viéitez C, Posas F, de Nadal E. 2010. The Rpd3L HDAC complex is essential for the heat stress response in yeast. Mol. Microbiol. 76:1049-1062.

Sadasivam S, DeCaprio JA. 2013. The DREAM complex: master coordinator of cell cycledependent gene expression. Nat. Rev. Cancer 13:585-595.

Schulz LL, Tyler JK. 2006. The histone chaperone ASF1 localizes to active DNA replication forks to mediate efficient DNA replication. FASEB J. 20:488-490. 
Shannon P, Markiel A, Ozier O, Baliga NS, Wang JT, Ramage D, Amin N, Schwikowski B, Ideker T. 2003. Cytoscape: a software environment for integrated models of biomolecular interaction networks. Genome Res. 13:2498-2504.

Sharp JA, Fouts ET, Krawitz DC, Kaufman PD. 2001. Yeast histone deposition protein Asf1p requires Hir proteins and PCNA for heterochromatic silencing. Curr. Biol. 11:463-473.

Sharp JA, Franco AA, Osley MA, Kaufman PD. 2002. Chromatin assembly factor I and Hir proteins contribute to building functional kinetochores in S. cerevisiae. Genes Dev. 16:85-100.

Sharp JA, Rizki G, Kaufman PD. 2005. Regulation of histone deposition proteins Asf1/Hir1 by multiple DNA damage checkpoint kinases in Saccharomyces cerevisiae. Genetics 171:885-899.

Sherwood PW, Tsang S V, Osley MA. 1993. Characterization of HIR1 and HIR2, two genes required for regulation of histone gene transcription in Saccharomyces cerevisiae. Mol. Cell. Biol. 13:28-38.

Shibahara K, Stillman B. 1999. Replication-dependent marking of DNA by PCNA facilitates CAF-1-coupled inheritance of chromatin. Cell 96:575-585.

Smith JJ, Torigoe SE, Maxson J, Fish LC, Wiley EA. 2008. A class II histone deacetylase acts on newly synthesized histones in Tetrahymena. Eukaryot. Cell 7:471-482.

Smith S, Stillman B. 1989. Purification and characterization of CAF-I, a human cell factor required for chromatin assembly during DNA replication in vitro. Cell 58:15-25.

Smith S, Stillman B. 1991. Immunological characterization of chromatin assembly factor I, a human cell factor required for chromatin assembly during DNA replication in vitro. J. Biol. Chem. 266:12041-12047.

Sobel RE, Cook RG, Allis CD. 1994. Non-random acetylation of histone H4 by a cytoplasmic histone acetyltransferase as determined by novel methodology. J. Biol. Chem. 269:18576-18582.

Sobel RE, Cook RG, Perry CA, Annunziato AT, Allis CD. 1995. Conservation of depositionrelated acetylation sites in newly synthesized histones H3 and H4. Proc. Natl. Acad. Sci. U. S. A. 92:1237-1241.

Song J-J, Garlick JD, Kingston RE. 2008. Structural basis of histone H4 recognition by p55. Genes Dev. [Internet] 22:1313-1318. Available from: http://www.pubmedcentral.nih.gov/articlerender.fcgi?artid=2377184\&tool=pmcentrez $\&$ rendertype $=$ abstract 
Song JY, Leung T, Ehler LK, Wang C, Liu Z. 2000. Regulation of meristem organization and cell division by TSO1, an Arabidopsis gene with cysteine-rich repeats. Development 127:2207-2217.

Song Y, Seol J-H, Yang J-H, Kim H-J, Han J-W, Youn H-D, Cho E-J. 2013. Dissecting the roles of the histone chaperones reveals the evolutionary conserved mechanism of transcription-coupled deposition of H3.3. Nucleic Acids Res. 41:5199-5209.

Sugihara T, Wadhwa R, Kaul SC, Mitsui Y. 1999. A novel testis-specific metallothioneinlike protein, tesmin, is an early marker of male germ cell differentiation. Genomics 57:130-136.

Suter B, Pogoutse O, Guo X, Krogan N, Lewis P, Greenblatt JF, Rine J, Emili A. 2007. Association with the origin recognition complex suggests a novel role for histone acetyltransferase Hat1p/Hat2p. BMC Biol. 5:38.

Taddei A, Roche D, Sibarita JB, Turner BM, Almouzni G. 1999. Duplication and maintenance of heterochromatin domains. J. Cell Biol. 147:1153-1166.

Tagami H, Ray-Gallet D, Almouzni G, Nakatani Y. 2004. Histone H3.1 and H3.3 complexes mediate nucleosome assembly pathways dependent or independent of DNA synthesis. Cell 116:51-61.

Tang Y, Poustovoitov M V, Zhao K, Garfinkel M, Canutescu A, Dunbrack R, Adams PD, Marmorstein R. 2006. Structure of a human ASF1a-HIRA complex and insights into specificity of histone chaperone complex assembly. Nat. Struct. Mol. Biol. 13:921-929.

Tang Y, Puri A, Ricketts MD, Rai TS, Hoffmann J, Hoi E, Adams PD, Schultz DC, Marmorstein R. 2012. Identification of an ubinuclein 1 region required for stability and function of the human HIRA/UBN1/CABIN1/ASF1a histone H3.3 chaperone complex. Biochemistry 51:2366-2377.

Taverna SD, Coyne RS, Allis CD. 2002. Methylation of histone h3 at lysine 9 targets programmed DNA elimination in tetrahymena. Cell 110:701-711.

Tian M, Chen X, Xiong Q, Xiong J, Xiao C, Ge F, Yang F, Miao W. 2014. Phosphoproteomic analysis of protein phosphorylation networks in Tetrahymena thermophila, a model single-celled organism. Mol. Cell. Proteomics 13:503-519.

Tjeertes J V, Miller KM, Jackson SP. 2009. Screen for DNA-damage-responsive histone modifications identifies H3K9Ac and H3K56Ac in human cells. EMBO J. 28:18781889.

Verreault A, Kaufman PD, Kobayashi R, Stillman B. 1996. Nucleosome assembly by a complex of CAF-1 and acetylated histones H3/H4. Cell 87:95-104. 
Vetting MW, S de Carvalho LP, Yu M, Hegde SS, Magnet S, Roderick SL, Blanchard JS. 2005. Structure and functions of the GNAT superfamily of acetyltransferases. Arch. Biochem. Biophys. 433:212-226.

Vignali M, Hassan AH, Neely KE, Workman JL. 2000. ATP-dependent chromatinremodeling complexes. Mol. Cell. Biol. 20:1899-1910.

Wang H, Ge Z, Walsh STR, Parthun MR. 2012. The human histone chaperone sNASP interacts with linker and core histones through distinct mechanisms. Nucleic Acids Res. 40:660-669.

Wang H, Walsh STR, Parthun MR. 2008. Expanded binding specificity of the human histone chaperone NASP. Nucleic Acids Res. 36:5763-5772.

Warde-Farley D, Donaldson SL, Comes O, et al. 2010. The GeneMANIA prediction server: biological network integration for gene prioritization and predicting gene function. Nucleic Acids Res. 38:W214-20.

Waterhouse AM, Procter JB, Martin DMA, Clamp M, Barton GJ. 2009. Jalview Version 2--a multiple sequence alignment editor and analysis workbench. Bioinformatics 25:11891191.

Wei Y, Mizzen CA, Cook RG, Gorovsky MA, Allis CD. 1998. Phosphorylation of histone $\mathrm{H} 3$ at serine 10 is correlated with chromosome condensation during mitosis and meiosis in Tetrahymena. Proc. Natl. Acad. Sci. U. S. A. 95:7480-7484.

Welch JE, Zimmerman LJ, Joseph DR, O'Rand MG. 1990. Characterization of a spermspecific nuclear autoantigenic protein. I. Complete sequence and homology with the Xenopus protein, N1/N2. Biol. Reprod. 43:559-568.

Wu H, Moshkina N, Min J, Zeng H, Joshua J, Zhou M-M, Plotnikov AN. 2012. Structural basis for substrate specificity and catalysis of human histone acetyltransferase 1. Proc. Natl. Acad. Sci. U. S. A. 109:8925-8930.

Xiong J, Yuan D, Fillingham JS, et al. 2011. Gene network landscape of the ciliate Tetrahymena thermophila. PLoS One 6:e20124.

Xu C, Min J. 2011. Structure and function of WD40 domain proteins. Protein Cell 2:202214.

Yamane K, Mizuguchi T, Cui B, Zofall M, Noma K, Grewal SIS. 2011. Asf1/HIRA facilitate global histone deacetylation and associate with HP1 to promote nucleosome occupancy at heterochromatic loci. Mol. Cell 41:56-66.

Yang X, Li L, Liang J, et al. 2013. Histone acetyltransferase 1 promotes homologous recombination in DNA repair by facilitating histone turnover. J. Biol. Chem. 288:18271-18282. 
Yao M-C, Choi J, Yokoyama S, Austerberry CF, Yao C-H. 1984. DNA elimination in tetrahymena: A developmental process involving extensive breakage and rejoining of DNA at defined sites. Cell 36:433-440.

Yao M-C, Fuller P, Xi X. 2003. Programmed DNA deletion as an RNA-guided system of genome defense. Science 300:1581-1584.

Yao MC, Yao CH, Monks B. 1990. The controlling sequence for site-specific chromosome breakage in Tetrahymena. Cell 63:763-772.

Zhang Y. 2008. I-TASSER server for protein 3D structure prediction. BMC Bioinformatics 9:40.

Zhong Y, Nellimoottil T, Peace JM, et al. 2013. The level of origin firing inversely affects the rate of replication fork progression. J. Cell Biol. 201:373-383.

Zhu B, Reinberg D. 2011. Epigenetic inheritance: uncontested? Cell Res. 21:435-441. 


\section{References (Part 2)}

Ai X, Parthun MR. 2004. The nuclear Hat1p/Hat2p complex: a molecular link between type B histone acetyltransferases and chromatin assembly. Mol. Cell 14:195-205.

Akashi H, Gojobori T. 2002. Metabolic efficiency and amino acid composition in the proteomes of Escherichia coli and Bacillus subtilis. Proc. Natl. Acad. Sci. U. S. A. 99:3695-3700.

Alekseev OM, Bencic DC, Richardson RT, Widgren EE, O'Rand MG. 2003. Overexpression of the Linker histone-binding protein tNASP affects progression through the cell cycle. J. Biol. Chem. 278:8846-8852.

Alekseev OM, Richardson RT, Tsuruta JK, O'Rand MG. 2011. Depletion of the histone chaperone tNASP inhibits proliferation and induces apoptosis in prostate cancer PC-3 cells. Reprod. Biol. Endocrinol. 9:50.

Ali-Fehmi R, Chatterjee M, Ionan A, et al. 2010. Analysis of the expression of human tumor antigens in ovarian cancer tissues. Cancer Biomark. 6:33-48.

Altenhoff AM, Dessimoz C. 2012. Inferring orthology and paralogy. Methods Mol. Biol. 855:259-279.

Alvarez F, Muñoz F, Schilcher P, Imhof A, Almouzni G, Loyola A. 2011. Sequential establishment of marks on soluble histones H3 and H4. J. Biol. Chem. 286:1771417721.

Ask K, Jasencakova Z, Menard P, Feng Y, Almouzni G, Groth A. 2012. Codanin-1, mutated in the anaemic disease CDAI, regulates Asf1 function in S-phase histone supply. EMBO J. 31:2013-2023.

Balaji S, Iyer LM, Aravind L. 2009. HPC2 and ubinuclein define a novel family of histone chaperones conserved throughout eukaryotes. Mol. Biosyst. 5:269-275.

Batova I, O'Rand MG. 1996. Histone-binding domains in a human nuclear autoantigenic sperm protein. Biol. Reprod. 54:1238-1244.

Bonner WM. 1975. Protein migration into nuclei. I. Frog oocyte nuclei in vivo accumulate microinjected histones, allow entry to small proteins, and exclude large proteins. J. Cell Biol. 64:421-430.

Campos EI, Fillingham J, Li G, et al. 2010. The program for processing newly synthesized histones H3.1 and H4. Nat. Struct. Mol. Biol. 17:1343-1351.

Chen B, Zhong D, Monteiro A. 2006. Comparative genomics and evolution of the HSP90 family of genes across all kingdoms of organisms. BMC Genomics 7:156. 
Cook AJL, Gurard-Levin ZA, Vassias I, Almouzni G. 2011. A Specific Function for the Histone Chaperone NASP to Fine-Tune a Reservoir of Soluble H3-H4 in the Histone Supply Chain. Mol. Cell 44:918-927.

D’Andrea LD, Regan L. 2003. TPR proteins: the versatile helix. Trends Biochem. Sci. 28:655-662.

Daganzo SM, Erzberger JP, Lam WM, et al. 2003. Structure and function of the conserved core of histone deposition protein Asf1. Curr. Biol. 13:2148-2158.

Dgany O, Avidan N, Delaunay J, et al. 2002. Congenital dyserythropoietic anemia type I is caused by mutations in codanin-1. Am. J. Hum. Genet. 71:1467-1474.

Dilworth SM, Black SJ, Laskey RA. 1987. Two complexes that contain histones are required for nucleosome assembly in vitro: role of nucleoplasmin and N1 in Xenopus egg extracts. Cell 51:1009-1018.

Dunleavy EM, Pidoux AL, Monet M, Bonilla C, Richardson W, Hamilton GL, Ekwall K, McLaughlin PJ, Allshire RC. 2007. A NASP (N1/N2)-related protein, Sim3, binds CENP-A and is required for its deposition at fission yeast centromeres. Mol. Cell 28:1029-1044.

Eirín-López JM, Frehlick LJ, Ausió J. 2006. Long-term evolution and functional diversification in the members of the nucleophosmin/nucleoplasmin family of nuclear chaperones. Genetics 173:1835-1850.

Eisen JA. 1998. Phylogenomics: improving functional predictions for uncharacterized genes by evolutionary analysis. Genome Res. 8:163-167.

Fillingham J, Recht J, Silva AC, et al. 2008. Chaperone control of the activity and specificity of the histone H3 acetyltransferase Rtt109. Mol. Cell. Biol. 28:4342-4353.

Finn RM, Browne K, Hodgson KC, Ausió J. 2008. sNASP, a histone H1-specific eukaryotic chaperone dimer that facilitates chromatin assembly. Biophys. J. 95:1314-1325.

Finn RM, Ellard K, Eirin Lopez JM, Ausio J. 2012. Vertebrate nucleoplasmin and NASP: egg histone storage proteins with multiple chaperone activities. FASEB J.

Foster PG, Jermiin LS, Hickey DA. 1997. Nucleotide composition bias affects amino acid content in proteins coded by animal mitochondria. J. Mol. Evol. 44:282-288.

Garg J, Lambert JP, Karsou A, et al. 2013. Conserved Asf1-importin ?? physical interaction in growth and sexual development in the ciliate Tetrahymena thermophila. J. Proteomics 94:311-326.

Grote P, Conradt B. 2006. The PLZF-like protein TRA-4 cooperates with the Gli-like transcription factor TRA-1 to promote female development in C. elegans. Dev. Cell 11:561-573. 
Karlin S, Blaisdell BE, Bucher P. 1992. Quantile distributions of amino acid usage in protein classes. Protein Eng. 5:729-738.

Kim H-S, Mukhopadhyay R, Rothbart SB, et al. 2014. Identification of a BET family bromodomain/casein kinase II/TAF-containing complex as a regulator of mitotic condensin function. Cell Rep. 6:892-905.

Kleinschmidt JA, Fortkamp E, Krohne G, Zentgraf H, Franke WW. 1985. Co-existence of two different types of soluble histone complexes in nuclei of Xenopus laevis oocytes. J. Biol. Chem. 260:1166-1176.

De Koning L, Corpet A, Haber JE, Almouzni G. 2007. Histone chaperones: an escort network regulating histone traffic. Nat. Struct. Mol. Biol. 14:997-1007.

Lei M. 2005. The MCM complex: its role in DNA replication and implications for cancer therapy. Curr. Cancer Drug Targets 5:365-380.

Luger K, Mäder AW, Richmond RK, Sargent DF, Richmond TJ. 1997. Crystal structure of the nucleosome core particle at 2.8 A resolution. Nature 389:251-260.

Ma W, Xie S, Ni M, Huang X, Hu S, Liu Q, Liu A, Zhang J, Zhang Y. 2012. MicroRNA-29a inhibited epididymal epithelial cell proliferation by targeting nuclear autoantigenic sperm protein (NASP). J. Biol. Chem. 287:10189-10199.

Nabeel-Shah S, Ashraf K, Pearlman RE, Fillingham J. 2014. Molecular evolution of NASP and conserved histone H3/H4 transport pathway. BMC Evol. Biol. 14:139.

O'Reilly AJ, Dacks JB, Field MC. 2011. Evolution of the karyopherin- $\beta$ family of nucleocytoplasmic transport factors; ancient origins and continued specialization. PLoS One 6:e19308.

Poveda A, Pamblanco M, Tafrov S, Tordera V, Sternglanz R, Sendra R. 2004. Hif1 is a component of yeast histone acetyltransferase $\mathrm{B}$, a complex mainly localized in the nucleus. J. Biol. Chem. 279:16033-16043.

Richardson RT, Alekseev OM, Grossman G, Widgren EE, Thresher R, Wagner EJ, Sullivan KD, Marzluff WF, O’Rand MG. 2006. Nuclear autoantigenic sperm protein (NASP), a linker histone chaperone that is required for cell proliferation. J. Biol. Chem. 281:21526-21534.

Richardson RT, Batova IN, Widgren EE, Zheng LX, Whitfield M, Marzluff WF, O'Rand MG. 2000. Characterization of the histone H1-binding protein, NASP, as a cell cycleregulated somatic protein. J. Biol. Chem. 275:30378-30386.

De Robertis EM, Partington GA, Gurdon JB. 1978. Selective gene expression by somatic nuclei injected into amphibian oocytes. Philos. Trans. R. Soc. Lond. B. Biol. Sci. 283:375-377. 
Rooney AP, Zhang J, Nei M. 2000. An unusual form of purifying selection in a sperm protein. Mol. Biol. Evol. 17:278-283.

Rooney AP. 2003. Selection for highly biased amino acid frequency in the TolA cell envelope protein of Proteobacteria. J. Mol. Evol. 57:731-736.

Sueoka N. 1961. Correlation between base composition of deoxyribonucleic acid and amino acid composition of protein. Proc. Natl. Acad. Sci. U. S. A. 47:1141-1149.

Sueoka N. 1988. Directional mutation pressure and neutral molecular evolution. Proc. Natl. Acad. Sci. U. S. A. 85:2653-2657.

Tagami H, Ray-Gallet D, Almouzni G, Nakatani Y. 2004. Histone H3.1 and H3.3 complexes mediate nucleosome assembly pathways dependent or independent of DNA synthesis. Cell 116:51-61.

Tanae K, Horiuchi T, Yamakawa T, Matsuo Y, Kawamukai M. 2012. Sim3 shares some common roles with the histone chaperone Asf1 in fission yeast. FEBS Lett. 586:41904196.

Thornton JW, DeSalle R. 2000. Gene family evolution and homology: genomics meets phylogenetics. Annu. Rev. Genomics Hum. Genet. 1:41-73.

Tong K, Keller T, Hoffman CS, Annunziato AT. 2012. Schizosaccharomyces pombe Hat1 (Kat1) is associated with Mis16 and is required for telomeric silencing. Eukaryot. Cell 11:1095-1103.

Wang H, Ge Z, Walsh STR, Parthun MR. 2012. The human histone chaperone sNASP interacts with linker and core histones through distinct mechanisms. Nucleic Acids Res. 40:660-669.

Wang H, Singer GAC, Hickey DA. 2004. Mutational bias affects protein evolution in flowering plants. Mol. Biol. Evol. 21:90-96.

Wang H, Walsh STR, Parthun MR. 2008. Expanded binding specificity of the human histone chaperone NASP. Nucleic Acids Res. 36:5763-5772.

Welch JE, Zimmerman LJ, Joseph DR, O’Rand MG. 1990. Characterization of a spermspecific nuclear autoantigenic protein. I. Complete sequence and homology with the Xenopus protein, N1/N2. Biol. Reprod. 43:559-568. 